\title{
Transport in Arrays of Submicron Josephson Junctions Over a Ground Plane
}

\author{
Teressa Rae Ho \\ (Ph.D. Thesis) \\ Department of Physics, University of California \\ and \\ Materials Sciences Division \\ Lawrenece Berkeley National Laboratory \\ University of California \\ Berkeley, CA 94720
}

December 1997

This work was supported by the Office of Basic Energy Sciences, Materials Sciences Division of the U.S. Department of Energy under contract number DE-AC03-76SF00098.

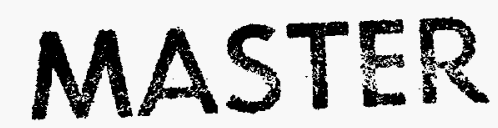

HH 


\section{DISCLAIMER}

This report was prepared as an account of work sponsored by an agency of the United States Government. Neither the United States Government nor any agency thereof, nor any of their employees, makes any warranty, express or implied, or assumes any legal liability or responsibility for the accuracy, completeness, or usefulness of any information, apparatus, product, or process disclosed, or represents that its use would not infringe privately owned rights. Reference herein to any specific commercial product, process, or service by trade name, trademark, manufacturer, or otherwise does not necessarily constitute or imply its endorsement, recommendation, or favoring by the United States Government or any agency thereof. The views and opinions of authors expressed herein do not necessarily state or reflect those of the United States Government or any agency thereof. 


\section{DISCLAIMER}

Portions of this document may be illegible electronic image products. Images are produced from the best available original document. 
Transport in Arrays of Submicron Josephson Junctions Over a Ground Plane

by

\author{
Teressa Rae Ho
}

B.A. (University of California at Berkeley) 1990

M.A. (University of California at Berkeley) 1994

A dissertation submitted in partial satisfaction of the requirements for the degree of

Doctor of Philosophy

in

Physics

in the

GRADUATE DIVISION

of the

UNIVERSITY of CALIFORNIA at BERKELEY

Committee in charge:

Professor John Clarke, Chair

Professor Paul McEuen

Professor David Chandler 


\title{
Transport in Arrays of Submicron Josephson Junctions Over a Ground Plane
}

\author{
Copyright $\odot 1997$ \\ by \\ Teressa Rae Ho
}

The Goverment reserves for itself and others acting on its behalf a royalty free, nonexclusive, irrevocable, world-wide license for govermental purposes to publish, distribute, translate, duplicate, exhibit, and perform any such data copyrighted by the contractor.

The U.S. Department of Energy has the right to use this document for any purpose whatsoever including the right to reproduce all or any part thereof

\section{MASTER}




\author{
Abstract \\ Transport in Arrays of Submicron Josephson Junctions Over a Ground Plane \\ by \\ Teressa Rae Ho \\ Doctor of Philosophy in Physics \\ University of California at Berkeley \\ Professor John Clarke, Chair
}

One-dimensional (1D) and two-dimensional (2D) arrays of $\mathrm{Al}$ islands linked by submicron $\mathrm{Al} / \mathrm{Al}_{\mathbf{x}} \mathrm{O}_{\mathbf{y}} / \mathrm{Al}$ tunnel junctions were fabricated on an insulating layer grown on a ground plane. The arrays were cooled to temperatures as low as $20 \mathrm{mK}$ where the Josephson coupling energy $E_{J}$ of each junction and the charging energy $E_{C}$ of each island were much greater than the thermal energy $k_{B} T$. The capacitance $C_{g}$ between each island and the ground plane was much greater than the junction capacitance $C$. Two classes of arrays were studied. In the first class, the normal state tunneling resistance of the junctions was much larger than the resistance quantum for single electrons, $R_{N} \gg R_{Q e} \equiv h / e^{2} \approx 25.8 \mathrm{k} \Omega$, and the islands were driven normal by an applied magnetic field such that $E_{J}=0$ and the array was in the Coulomb blockade regime. The arrays were made on degenerately-doped $\mathrm{Si}$, thermally oxidized to a thickness of approximately $100 \mathrm{~nm}$. The current-voltage $(I-V)$ characteristics of a $1 \mathrm{D}$ and a $2 \mathrm{D}$ array were measured and found to display a threshold voltage $V_{T}$ below which little current flows. Above threshold the array current $I$ scaled as $\left(V / V_{T}-1\right)^{\zeta}$; this scaling behavior was interpreted as a dynamic critical phenomenon. A 2D array with intentionally-added island area disorder was also measured and found to have a similar threshold voltage $V_{T}$ but a larger scaling exponent $\zeta$ than the array with only intrinsic disorder.

In the second class of arrays, the normal state tunneling resistance of the junctions was close to the resistance quantum for Cooper pairs, $R_{N} \approx R_{Q} \equiv h / 4 e^{2} \approx 6.45 \mathrm{k} \Omega$, such that $E_{J} / E_{C} \approx 1$. The arrays were made on $\mathrm{GaAs} / \mathrm{Al}_{0.3} \mathrm{Ga}_{0.7} \mathrm{As}$ heterostructures with a two-dimensional electron gas (2DEG) approximately $100 \mathrm{~nm}$ below the surface. 
The resistance per square of the $2 \mathrm{DEG}, R_{g}$, could be varied by applying a large voltage between the $2 \mathrm{DEG}$ and a metallic back gate; varying $R_{g}$ varied the dissipation associated with the local electrodynamic environment. For a $2 \mathrm{D}$ array, the $I-V$ characteristics made a transition from superconductor-like to insulator-like as the resistance of the ground plane $R_{g}$ was increased. The zero-bias resistance $R_{0}$ of the array increased exponentially with $R_{g}$. A small magnetic field was applied perpendicularly to the array, and the $I$ $V$ characteristics of the array changed from superconductor-like to insulator-like as the magnetic field (measured in units of frustration $f$, the number of flux quanta per unit cell) was increased. Increasing $R_{g}$ drove the magnetic field-driven superconductor-like to insulator-like transition of the $I-V$ characteristics to lower values of $f$, and increasing $f$ drove the dissipation-driven transition to lower values of $R_{g}$. Three $1 \mathrm{D}$ arrays were also measured. One array displayed superconducting behavior at low temperature, and the size of the supercurrent increased, reached a maximum, and then decreased, as $R_{g}$ was increased. Two arrays displayed insulating behavior at low temperature, and the size of the Coulomb gap increased with increasing $R_{g}$. The zero-bias resistance of the array $R_{0}$ increased faster than exponentially with $R_{g}$. 
To my parents,

Fuhuan and Bin-Lun Ho 



\section{Contents}

List of Figures viii

List of Tables $\quad$ xi

I Introductory Material 1

1 Introduction 2

2 Single Tunnel Junction 5

2.1 Introduction . . . . . . . . . . . . . . . . 5

2.2 Single Junction Hamiltonian . . . . . . . . . . . . . . . . 5

2.3 Single Superconducting Tunnel Junction . . . . . . . . . . . . . . 8

2.4 Single Normal Metal Tunnel Junction . . . . . . . . . . . . . . . 8

3 One-Dimensional and Two-Dimensional Arrays of Normal Metal Tunnel Junctions 10

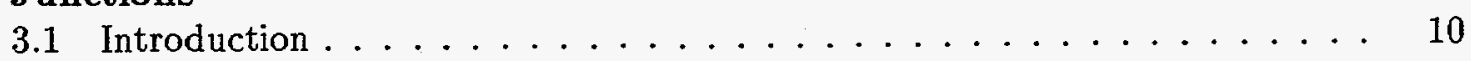

3.2 One-Dimensional Arrays of Normal Metal Junctions . . . . . . . . . . . . . 10

3.2.1 Gibbs Free Energy and Tunneling Probability . . . . . . . . . . . 11

3.2 .2 Statics - Threshold Voltage . . . . . . . . . . . . . . . . 13

3.2 .3 Dynamics - Offset Voltage . . . . . . . . . . . . . . . . . . 13

3.3 Two-Dimensional Arrays of Normal Metal Junctions . . . . . . . . . . . . . . . 14

3.3.1 Gibbs Free Energy and Tunneling Probability ... . . . . . . . . . 15

3.3.2 Statics - Two-Dimensional Solitons .................. 16

3.3.3 Dynamics - Offset Voltage . . . . . . . . . . . . . . 16

3.4 One-Dimensional and Two-Dimensional Arrays of Normal Metal Islands with Offset Charges ... . . . . . . . . . . . . . . . . 17

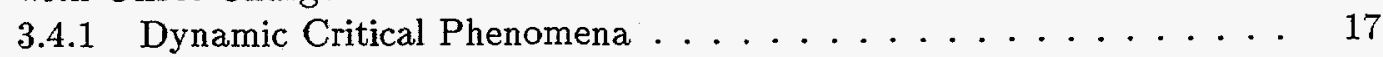

3.4 .2 Electrostatic Energy . . . . . . . . . . . . . . . . . . . 18

3.4 .3 Threshold Voltage . . . . . . . . . . . . . . . . . . . . 19

3.4 .4 Interface Motion . . . . . . . . . . . . . . . . . . . . . 19

3.4 .5 Current-Voltage Characteristics .............. 20 
4 One-Dimensional and Two-Dimensional Arrays of Josephson Junctions 22

4.1 Introduction . . . . . . . . . . . . . . . . 22

4.2 One-Dimensional Arrays of Josephson Junctions . . . . . . . . . . . . . . . . 22

4.2 .1 Hamiltonian . . . . . . . . . . . . . . . . . 23

4.2.2 Quantum Fluctuations in One-Dimensional Arrays of Josephson Junc-

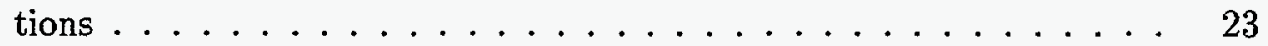

4.2.3 Dissipation in One-Dimensional Josephson Junction Arrays . . . . . 24

4.3 Two-Dimensional Arrays of Josephson Junctions . . . . . . . . . . . . . 24

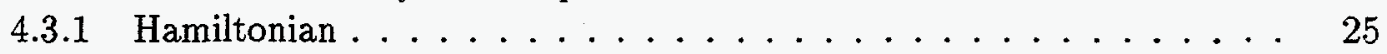

4.3.2 Applied Magnetic Field and Frustration . . . . . . . . . . . 26

4.3.3 Dissipation and Superconductor-Insulator Transitions . . . . . . . 28

5 Superconductor-Insulator Transitions in Arrays of Josephson Junctions 29

5.1 Introduction . . . . . . . . . . . . . . . 29

5.2 Quantum Phase Transitions . . . . . . . . . . . . . . . . 29

5.3 Charging Energy-Driven Superconductor-Insulator Transition . . . . . . . . 31

5.4 Magnetic Field-Driven Superconductor-Insulator Transition . . . . . . . . 32

5.5 Dissipation-Driven Superconductor-Insulator Transition . . . . . . . . . . 33

5.5 .1 Shunt Resistors . . . . . . . . . . . . . . . . 34

5.5 .2 Quasiparticle Tunneling ................... 35

5.5 .3 Ground Plane . . . . . . . . . . . . . . . . 36

II Experimental Techniques $\quad 39$

6 Sample Preparation $\quad 41$

6.1 Substrate Preparation Techniques . . . . . . . . . . . . . . . . 41

6.1 .1 Silicon Substrates ...................... 41

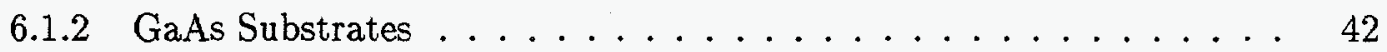

6.2 Array Fabrication Techniques . . . . . . . . . . . . . 45

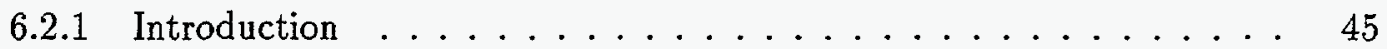

6.2 .2 Electron-Beam Lithography . . . . . . . . . . . . . . . . 47

6.2 .3 Shadow Evaporation .................... 56

7 Dilution Refrigerator $\quad \mathbf{5 9}$

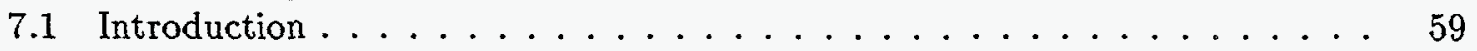

7.2 General Configuration . . . . . . . . . . . . . . . . . . 59

7.2 .1 Heat Sinks .......................... 60

7.3 Sample Mount . . . . . . . . . . . . . . . . 61

7.3.1 Sample Holder . . . . . . . . . . . . . . . . . 61

7.3 .2 Microwave Filters . . . . . . . . . . . . . . . . . . 63

7.3.3 Ruthenium Oxide Thermometer ............... 64

7.4 Thermometry and Temperature Control . . . . . . . . . . . . . 64

7.5 Superconducting Magnet . . . . . . . . . . . . . 65 
8 Measurement Techniques $\quad 66$

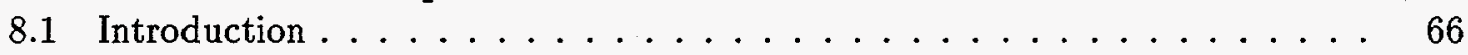

8.2 Array Measurements . . . . . . . . . . . . . . . . . . . . 67

8.2.1 I-V Characteristics of Array . . . . . . . . . . . . . . 67

8.2.2 Zero-Bias Resistance of Array . . . . . . . . . . . 68

8.2.3 Island Capacitance to Ground . . . . . . . . . . . . . . . . 68

8.3 Ground Plane Characterization . . . . . . . . . . . . . . . . . 69

8.3.1 Ground Plane Resistivity . . . . . . . . . . . . . . 69

8.3.2 Sheet Density of $2 \mathrm{DEG} \ldots \ldots \ldots \ldots \ldots$

8.4 Magnetic Field Sweeps and Ramping . . . . . . . . . . . . . . . 69

8.5 Data Acquisition and Analysis . . . . . . . . . . . 70

$\begin{array}{ll}\text { III Measurement and Discussion of Arrays } & \mathbf{7 1}\end{array}$

9 Scaling Behavior in the Current-Voltage Characteristic of One- and TwoDimensional Arrays of Tunnel Junctions $\quad \mathbf{7 2}$

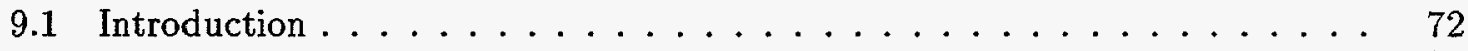

9.2 Middleton and Wingreen Model . . . . . . . . . . . . . 73

9.3 Description of Samples . . . . . . . . . . . . . . . . . . 74

9.4 Description of Experimental Setup and Measurements . . . . . . . . 74

9.5 Characterization of the Array . . . . . . . . . . . . . . . . 75

9.6 Current-Voltage Characteristics of Array . . . . . . . . . . 77

9.6.1 Threshold Voltage ... . . . . . . . . . . . . . . . 77

9.6 .2 Scaling Exponent . . . . . . . . . . . . . . . 78

9.7 Discussion . . . . . . . . . . . . . . . . . . . 80

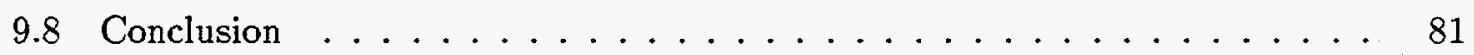

10 Two-Dimensional Array of Tunnel Junctions With Specifically Intro$\begin{array}{lr}\text { duced Disorder } & 82\end{array}$

10.1 Introduction . . . . . . . . . . . . . . . . . . . 82

10.2 Description of Array . . . . . . . . . . . . . . . . . . 83

10.3 Characterization of Island Area Disorder . . . . . . . . . . . . . . 84

10.4 Current-Voltage Characteristics of Array . . . . . . . . . 85

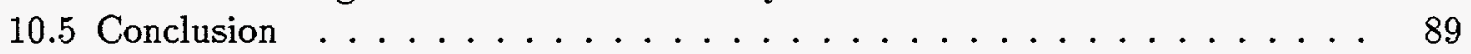

11 Dissipation-Driven Superconductor-Insulator Transition in a Two-Di$\begin{array}{ll}\text { mensional Josephson Junction Array } & 91\end{array}$

11.1 Introduction . . . . . . . . . . . . . . . . . . . . 91

11.2 Description of Sample . . . . . . . . . . . . . . . . . 92

11.3 Superconductor-Insulator Transitions in Arrays of Josephson Junctions . . 94

11.4 Experimental Setup and Sample Characterization . . . . . . . . . . . . 95

11.5 Current-Voltage Characteristics of Array . . . . . . . . . . . . . 96

11.6 Effects of Varying Ground Plane Resistance on Parameters of Array . . . . 97

11.7 Zero-Bias Resistance of Array . . . . . . . . . . . . . . . 98 
11.8 Comparison of Results to Theory . . . . . . . . . . . . . . . . 98

11.9 Conclusion . . . . . . . . . . . . . . . . . 102

12 Magnetic Field Dependence of a Dissipation-Driven SuperconductorInsulator Transition in a Two-Dimensional Array of Josephson Junctions 103 12.1 Introduction . . . . . . . . . . . . . . . . 103

12.2 Description of Sample . . . . . . . . . . . . . . . . . . . . . . . . . . . . . . . . . . . . . . .

12.3 Description of Experiment . . . . . . . . . . . . . . . . . . 104

12.4 Current-Voltage Characteristics of Array at Smallest Ground Plane Resistance104

12.5 Zero-Bias Resistance of Array at Smallest Ground Plane Resistance . . . . 106

12.6 Zero-Bias Resistance of Array at Different Values of Ground Plane Resistance108

12.7 Ground Plane Resistance Versus Frustration Phase Diagram . . . . . . . . . 108

12.8 Scaling Behavior of the Magnetic Field-Driven Superconductor-Insulator

Transition at the Smallest Ground Plane Resistance . . . . . . . . . . 112

12.9 Conclusion . . . . . . . . . . . . . . . . . 113

13 One-Dimensional Arrays of Josephson Junctions with Tunable Dissipation

13.1 Introduction . . . . . . . . . . . . . . . 115

13.2 Description of Samples . . . . . . . . . . . . . . 116

13.2.1 Description of Arrays . . . . . . . . . . . . 116

13.2.2 Description of Ground Planes . . . . . . . . . . . . 118

13.3 Current-Voltage Characteristics . . . . . . . . . . . . . . 118

13.3.1 General Properties . . . . . . . . . . . . . . . . . . . . 119

13.3.2 Effect of Dissipation on Supercurrent . . . . . . . . . . . 119

13.3.3 Effect of Dissipation on Coulomb Gap . . . . . . . . . . . . 123

13.4 Zero-Bias Resistance of Arrays . . . . . . . . . . . . . . . 124

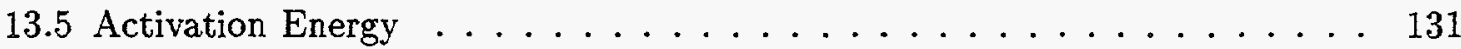

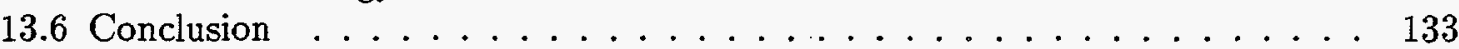

14 Conclusions and Future Directions

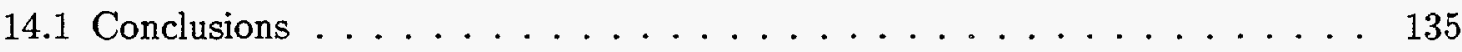

14.2 Future Directions . . . . . . . . . . . . . . . . . 137

A Preparation of Degenerately-Doped Silicon Substrates 138

B Electron-Beam Lithography Exposure Procedure 141

C Two-Dimensional Josephson Junction Arrays and the XY Model 143

Bibliography 144 


\section{List of Figures}

2.1 Schematic drawing of a single Josephson junction and two commonly used symbols for a Josephson junction. . . . . . . . . . . . . 6

3.1 Schematic diagram of a 1D array of tunnel junctions over a ground plane. - 11

3.2 Schematic diagram of a $2 \mathrm{D}$ array of tunnel junctions over a ground plane. . 14

3.3 Schematic diagram of the function $\alpha$ versus the ratio $C / C_{g}$ from Middleton

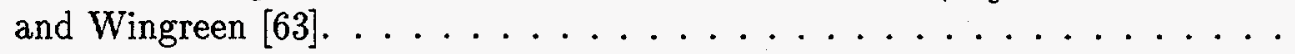

4.1 From Bobbert et al. [9]; $T=0$ phase diagram in the $J=\left(E_{J} / 8 E_{C}\right)^{1 / 2}$

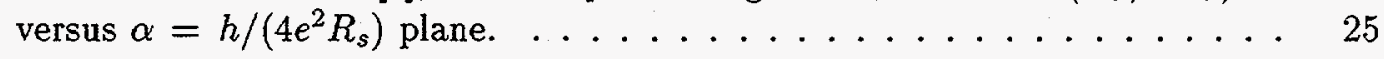

4.2 Vortex lattice for frustrations $f=1 / 2$ and $1 / 3 \ldots \ldots \ldots \ldots \ldots$

5.1 Predictions of Wagenblast et al. for the zero-bias resistance of an array, in units of the resistance quantum $R_{Q}$, versus temperature for six different values of the ratio $\alpha=R_{Q} / R_{2 D E G} \ldots \ldots \ldots \ldots$

6.1 Schematic drawing of a degenerately-doped Si substrate with $100 \mathrm{~nm}$ oxide and $\mathrm{Al}$ contact pads. . . . . . . . . . . . . . . . . 42

6.2 Band diagram of a 2DEG in a GaAs/AlGaAs heterostructure. . . . . . . . 43

6.3 The three basic steps of electron-beam lithography and shadow evaporation. 46

6.4 Electron-beam lithography sample pattern, including the array, intermediate leads, and contact pads. . . . . . . . . . . . . 50

6.5 SEM micrograph of a $2 \mathrm{D}$ array with 38 columns and 40 rows of islands. . . 51

6.6 SEM micrograph of an island from a $38 \times 40$ island $2 \mathrm{D}$ array. . . . . . . 52

6.7 SEM micrograph of a single junction. . . . . . . . . . . . . 53

6.8 Schematic drawings of a small $1 \mathrm{D}$ array. . . . . . . . . . . 54

7.1 Dilution refrigerator sample mount, showing sample holder, $\mathrm{RuO}_{2}$ thermometer, $\mathrm{Nb}$ shield, and cancellation coil. . . . . . . . . . . . . .

7.2 Front, side, and top view of a sample holder ( $2 \times$ actual size) showing the location of a mounted substrate and back gate. . . . . . . . 63

8.1 Circuit for measuring the DC $I-V$ characteristics of an array. . . . . 67 
9.1 I $-V$ characteristics of the (a) $1 \mathrm{D}$ and (b) $2 \mathrm{D}$ arrays with the islands in the

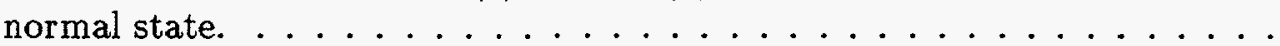

9.2 Typical composite curves of current vs. reduced voltage $\left(V / V_{T}-1\right)$ plotted

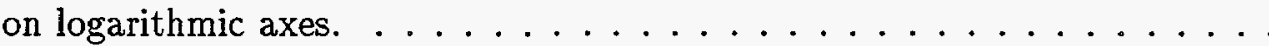

10.1 SEM micrograph of a portion of a $2 \mathrm{D}$ array with intentionally-added island area disorder.

10.2 Array voltage $V$ versus front gate voltage $V_{F G}$ for arrays with (a) intrinsic disorder and (b) area disorder.

10.3 Power spectral density of array voltage $V$ versus front gate voltage $V_{F G}$ in units of island capacitance $C_{g}=e / \Delta V_{F G}$ for arrays with (a) intrinsic disorder and (b) area disorder.

10.4 $I-V$ characteristics of arrays with (a) intrinsic disorder and (b) area disorder at $T=20 \mathrm{mK}$.

10.5 Scaling behavior of the $I-V$ characteristics of the $2 \mathrm{D}$ arrays with (a) intrinsic disorder and $(b)$ area disorder. . . . . . . . . . . . . .

11.1 Schematic diagram of an array with variable dissipation. . . . . . . .

$11.2 I-V$ characteristics of the array at zero magnetic field and $T=25 \mathrm{mK}$ for eight back gate voltages $V_{B G}=0,100,200,300,400,450,500$, and 525 $\mathrm{V}$, corresponding to ground plane resistances $R_{g}=170,240,300,570,990$, 1350,1900 , and $2290 \Omega / \mathrm{sq}$, respectively. . . . . . . . . .

11.3 Zero-bias resistance $R_{0}$ of the array versus the resistance per square $R_{g}$ of the ground plane for four temperatures $T=25,50,100$, and $150 \mathrm{mK}$. . .

11.4 Predictions of Wagenblast et al. for the zero-bias resistance of an array, in units of the resistance quantum $R_{Q}$, versus temperature for six different values of the ground plane resistance $R_{g}=200,700,1200,1700,2200$, and $2700 \Omega /$ sq.

11.5 Predictions of Wagenblast et al. for the zero-bias resistance of the array described in Fig. 11.4, in units of the resistance quantum $R_{Q}$, versus ground plane resistance $R_{g}\left(R_{g} \equiv R_{2 D E G}\right)$ for four different temperatures $T=25$,

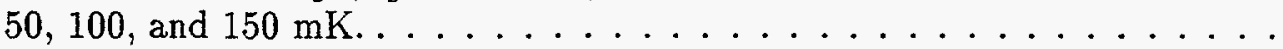

11.6 Phase diagram from Wagenblast et al. for an array capacitively coupled to a 2DEG.

12.1 Current-voltage characteristics of the array at ground plane resistance $R_{g}=$ $170 \Omega / \mathrm{sq}$ and $T=20 \mathrm{mK}$ for seven different frustrations $f=0,0.007,0.025$, $0.05,0.1,0.2$, and 0.37 .

12.2 Zero-bias resistance $R_{0}$ of the array versus frustration $f$ at the smallest value of resistance per square of the ground plane $R_{g}=170 \Omega / \mathrm{sq}$ for three temperatures $T=25,44$, and $75 \mathrm{mK} \ldots \ldots \ldots \ldots$ 
12.4 Zero-bias resistance $R_{0}$ of the array versus frustration $f$ for ten different values of the ground plane resistance $R_{g}=170,200,240,290,360,450,570$, 740,990 , and $1350 \Omega / \mathrm{sq}$, from bottom to top. . . . . . . . .

12.5 Zero-bias resistance $R_{0}$ of the array versus the resistance per square of the ground plane $R_{g}$ for four frustrations $f=0,0.002,0.004$, and 0.006 , from bottom to top.

12.6 Phase diagram for the superconductor-like to insulator-like transition of the $I-V$ characteristics of the array in the ground plane resistance $R_{g}$ versus

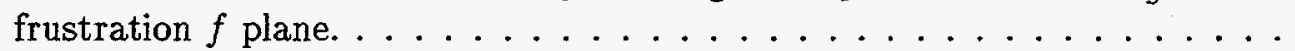

12.7 Results of the scaling analysis for the magnetic field-driven superconductorinsulator transition of the array for data from $T=75$ to $400 \mathrm{mK}$. . . . .

$13.1 I-V$ characteristics of the $1 \mathrm{D}$ arrays at $T=20 \mathrm{mK}$, zero magnetic field, and $V_{B G}=0\left(R_{g}=110-120 \Omega / \mathrm{sq}\right) . \ldots \ldots \ldots$

$13.2 I-V$ characteristics of sample $\mathrm{A}\left(R_{N}=2.75 \mathrm{k} \Omega\right)$ at $T=20 \mathrm{mK}$ and zero magnetic field for four different ground plane resistances $R_{g}=110,1060$, $12.2 \mathrm{k}$, and $>1 \mathrm{G} \Omega / \mathrm{sq}$, corresponding to $V_{B G}=0,300,400$, and $450 \mathrm{~V}$.

13.3 Critical current $I_{c}$ of the array versus ground plane resistance $R_{g}$ for sample $\mathrm{A}$ at $\mathrm{T}=20 \mathrm{mK} \ldots \ldots \ldots \ldots \ldots$

13.4 $I-V$ characteristics of sample C $\left(R_{N}=17.6 \mathrm{k} \Omega\right)$ at $T=20 \mathrm{mK}$ and zero magnetic field for six different ground plane resistances $R_{g}=110,1220,9760$, $32.1 \mathrm{k}, 1.22 \mathrm{M}$, and $>1 \mathrm{G} \Omega / \mathrm{sq}$, corresponding to $V_{B G}=0,450,600,625$,

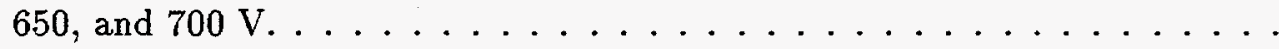

13.5 Threshold voltage $V_{T}$ of the array versus ground plane resistance $R_{g}$ for samples (a) B and (b) C.

13.6 Zero-bias resistance of the array $R_{0}$ versus temperature $T$ for samples (a) A, (b) B, and (c) C at zero applied magnetic field for four different values of the ground plane resistance $R_{g} \ldots \ldots \ldots \ldots \ldots$

13.7 Zero-bias resistance of the array $R_{0}$ versus temperature $T$ for samples $A$ and $\mathrm{C}$ at zero applied magnetic field for four different values of the ground plane

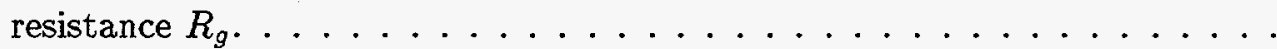

13.8 Zero-bias resistance of the array $R_{0}$ versus temperature $T$ for samples $A$ and $\mathrm{B}$ for $0.4 \mathrm{~T}$ applied magnetic field (islands in the normal state) for different values of the ground plane resistance $R_{g} \ldots \ldots \ldots \ldots$

13.9 Zero-bias resistance of the array $R_{0}$ versus ground plane resistance $R_{g}$ for sample $A$ at zero applied magnetic field for fifteen different values of temperature $T=20,100,150,200,250,300,350,400,450,500,550,600,650,700$, and $800 \mathrm{mK}$, from bottom to top. . . . . . . . . . . .

13.10Determination of the activation energy $E_{a}$ for sample $C$ with the islands of the array in the superconducting state (zero applied magnetic field). . . . . 


\section{List of Tables}

6.1 Parameters of the 2DEGs in the GaAs/AlGaAs heterostructures at $V_{B G}=$ 0 and $T=20 \mathrm{mK} \ldots \ldots \ldots \ldots \ldots \ldots \ldots \ldots$

6.2 Typical SEM exposure parameters for the sample pattern for accelerating

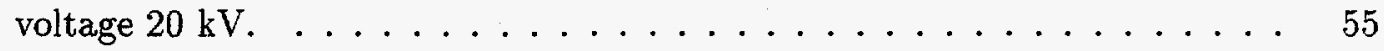

13.1 Parameters of the three $1 \mathrm{D}$ arrays. . . . . . . . . . . . . 117

13.2 Activation energies for samples $\mathrm{A}, \mathrm{B}$, and $\mathrm{C}$ for zero magnetic field (superconducting islands) $\left(E_{a S}\right)$ and $0.4 \mathrm{~T}$ magnetic field (normal islands) $\left(E_{a N}\right) . \quad 131$ 


\section{Acknowledgements}

Firstly, I would like to thank my advisor, Professor John Clarke, for his unfailing encouragement, support, and guidance, and for the opportunity to work on many exciting projects in his laboratory.

I would also like to thank Alex Rimberg for his patience, perseverance, and humor. He taught me the fine art of electron-beam lithography in addition to many other experimental techniques, and was always willing to answer any questions $I$ had in a manner that I could understand. With his guidance, I learned how to be a good scientist. I will also never forget his amazing sound effects. I would like to thank Çağliyan Kurdak for his guidance in my later years of graduate research. His enthusiasm and readiness to help or answer questions was nearly unbelievable. He was always there to encourage me, and his eggplant recipes were excellent. I would also like to thank the latest addition to the Submicron Group, Roy Therrien, for his undying curiosity, hard-working nature, and appreciation of 80's music. Although I only overlapped briefly with John Schmidt, I thank him for cheerfully accepting random phonecalls and emails from myself and others in the Submicron Group, and for all the work he has done on the fridge.

I thank the staff of the Berkeley Physics Department, the Machine Shop, the Electronics Shop, the Stockroom, the Student Assistants, and others, for all their help during both my graduate and undergraduate years. I thank the staff and members of the Berkeley Microfabrication Laboratory for their friendliness and help with silicon processing.

I thank all the members of the Clarke Group, past and present, for their company and comradery, and for making graduate school a fun place to be.

Finally, I would like to thank my parents and brothers for their love and support throughout my lengthy education. 
Part I

Introductory Material 


\section{Chapter 1}

\section{Introduction}

Arrays of submicron Josephson junctions over a ground plane are model systems for studying a variety of physical phenomena such as dynamic critical phenomena [63] and superconductor-to-insulator transitions [86]. The arrays consist of a regular pattern of small superconducting islands linked by very thin insulating layers that form the Josephson junctions. The arrays are fabricated on substrates that contain a conducting region, the ground plane, separated from the array by an insulating layer. Modern nanofabrication techniques allow the arrays to be fabricated with very specific parameters. The characteristic parameters of the arrays, the normal state tunneling resistance $R_{N}$ and the capacitance $C$ of the junctions, and the capacitance to ground $C_{g}$ of the islands, can all be tailored to study a particular phenomenon. In this work, the significance of another parameter in the system, the resistance per square of the ground plane $R_{g}$, is also investigated.

Josephson junction arrays are characterized by two energies, the Josephson energy $E_{J}$ which describes the superconducting coupling energy of the junctions, and the charging energy $E_{C}$, which describes the energy to transfer an electron between neighboring islands. At low temperatures $\left(E_{J}, E_{C} \ll k_{B} T\right), E_{J}$ is a function of only $R_{N}$ and the superconducting energy gap $\Delta$ of the island material, and $E_{C}$ is a function only of $C$ and $C_{g}$. Extensive experimental work has been performed on Josephson junction arrays with no ground plane (for example, see [86]). In these works, it was found that arrays with a large ratio $E_{J} / E_{C}$ displayed superconducting behavior at low temperatures $(<100 \mathrm{mK})$, while those with a small $E_{J} / E_{C}$ ratio displayed insulating behavior. This superconductorto-insulator (S-I) transition of the array as a function of $E_{J} / E_{C}$ occurs at higher values of $E_{J} / E_{C}$ in arrays with a ground plane, because dissipation associated with the local elec- 
trodynamic environment due to the ground plane will cause damping of the fluctuations of the superconducting phase across the junctions $[15,32]$. The islands of the arrays can be driven normal with an applied magnetic field so that $E_{J}=0$. For normal metal arrays with normal state tunneling resistance much larger than the resistance quantum for single electrons, $R_{N} \gg R_{Q e} \equiv h / e^{2} \approx 25.8 \mathrm{k} \Omega$, such that $E_{C}$ is much larger than the energy scale of quantum fluctuations, charging effects in the arrays can be observed.

In this dissertation, studies of one-dimensional (1D) and two-dimensional (2D) arrays of submicron $\mathrm{Al} / \mathrm{Al}_{\mathrm{x}} \mathrm{O}_{\mathrm{y}} / \mathrm{Al}$ tunnel junctions over a ground plane are presented. In our early work we studied $1 \mathrm{D}$ and $2 \mathrm{D}$ arrays of normal metal islands linked by tunnel junctions with $R_{N} \gg R_{Q e}\left(E_{J}=0\right)$ over a ground plane. This work was motivated by theoretical work of Middleton and Wingreen [63] describing dynamic critical phenomena and scaling behavior in the current-voltage characteristics of such arrays. Later, we studied $1 \mathrm{D}$ and $2 \mathrm{D}$ arrays of superconducting islands linked by Josephson junctions with $R_{N}$ close to the resistance quantum for Cooper pairs, $R_{N} \approx R_{Q} \equiv h / 4 e^{2} \approx 6.45 \mathrm{k} \Omega\left(E_{J} / E_{C} \approx 1\right)$ over a ground plane with variable resistance. Varying the ground plane resistance $R_{g}$ varies the dissipation associated with the local electrodynamic environment without changing other relevant physical parameters of the system. In these arrays, the superconductor-like to insulator-like transition of the array is investigated as a function of dissipation due to a ground plane.

In Part I, the theory of arrays of normal metal and superconducting tunnel junctions is summarized. Chapter 2 reviews the theory of single normal metal and superconducting tunnel junctions. In Chapter 3 , the theory of $1 \mathrm{D}$ and $2 \mathrm{D}$ arrays of normal metal tunnel junctions is described, with emphasis on the work of Middleton and Wingreen describing the scaling behavior of the current-voltage characteristics of arrays with $R_{N} \gg R_{Q e}$ and $C_{g} \gg C$ over a ground plane. In Chapter 4 , the theory of $1 \mathrm{D}$ and $2 \mathrm{D}$ arrays of superconducting tunnel junctions is described, with a discussion of the effects of quantum fluctuations in $1 \mathrm{D}$ arrays and an applied magnetic field in $2 \mathrm{D}$ arrays. Chapter 5 discusses superconductor-to-insulator transitions in arrays of Josephson junctions driven by charging energy, applied magnetic field, and dissipation, with emphasis on the work of Wagenblast et al. [93] describing a transition driven by dissipation due to a ground plane.

In Part II, the techniques of our experiments are described. Chapter 6 describes the preparation of the degenerately-doped $\mathrm{Si}$ and $\mathrm{GaAs} / \mathrm{Al}_{0.3} \mathrm{Ga}_{0.7} \mathrm{As}$ substrates and the fabrication of the arrays by electron-beam lithography and shadow evaporation $[24,42]$. 
Chapter 7 describes the dilution refrigerator used to perform low temperature measurements of the arrays of Josephson junctions at temperatures $T \geq 20 \mathrm{mK}$. Chapter 8 discusses our measurement techniques, including a description of array measurements, ground plane characterization, and magnetic field sweeps and ramping.

Part III describes our experiments on $1 \mathrm{D}$ and $2 \mathrm{D}$ arrays of normal metal and superconducting tunnel junctions over a ground plane with $C_{g} \gg C$. In Chapter 9 is described the scaling behavior in the current-voltage characteristics of a $1 D$ and $2 \mathrm{D}$ array of normal metal islands linked by tunnel junctions with $R_{N} \gg R_{Q e}$, with a comparison of our results with the theoretical predictions of Middleton and Wingreen. Chapter 10 discusses our measurements of a $2 \mathrm{D}$ array of normal metal islands linked by tunnel junctions with $R_{N} \gg R_{Q e}$ with intentionally-added island area disorder; the results are compared to the Middleton and Wingreen theory and to the results for the arrays with no added island area disorder discussed in Chapter 10. In Chapter 11, our study of a 2D array of superconducting islands linked by Josephson junctions with $R_{N} \approx R_{Q}\left(E_{J} / E_{C} \approx 1\right)$ over a ground plane plane with variable resistance $R_{g}$, and thus variable dissipation, is described, including the observation of a dissipation-driven superconductor-like to insulator-like transition of the current-voltage characteristics of the array. Chapter 12 discusses the combined effects of varying $R_{g}$ and the applied magnetic field on the properties of the array described in Chapter 11. Chapter 13 describes our study of three $1 D$ arrays of Josephson junctions with $R_{N} \approx R_{Q}$ over a ground plane with variable dissipation, including a discussion of the change in the current-voltage characteristics and zero-bias resistance of the arrays as the ground plane resistance is increased. Chapter 14 gives a conclusion of the work presented in this dissertation, with a proposal for future work. 


\section{Chapter 2}

\section{Single Tunnel Junction}

\subsection{Introduction}

This chapter discusses the theory of single tunnel junctions. In Sec. 2.2, the Hamiltonian for a single tunnel junction is discussed, with a description of the Josephson energy $E_{J}$ and charging energy $E_{C}$ of a junction. In Sec. 2.3 and Sec. 2.4, the theory of single superconducting and normal metal tunnel junctions, respectively, is discussed.

\subsection{Single Junction Hamiltonian}

A tunnel junction is two metal or superconducting electrodes separated by a thin insulating barrier. A superconductor-insulator-superconductor tunnel junction (SIS junction) is called a "Josephson junction", named after the man who predicted its properties in 1962, Brian D. Josephson [48]. The behavior exhibited by a Josephson junction can also be seen in other "weak link" systems such as two superconductors separated by a thin normal metal layer or a point contact between superconductors.

Shown in Fig. 2.1 is a schematic drawing of a single Josephson junction. A Ginzburg-Landau order parameter $\Phi_{1,2}=\left|\Phi_{1,2}\right| e^{i \phi_{1,2}}$ describes the superconducting state of each superconducting electrode. If both electrodes are made of the same material, $\left|\Phi_{1}\right|=\left|\Phi_{2}\right|$. There is a phase difference $\phi=\phi_{1}-\phi_{2}$ across the junction. The junction has a capacitance $C$ and charge $Q$.

The Hamiltonian for the junction is given by the following expression at $\mathrm{T}=0[83]$ :

$$
H(\phi, Q)=H_{0}+H_{b}+H_{r}
$$


(a)

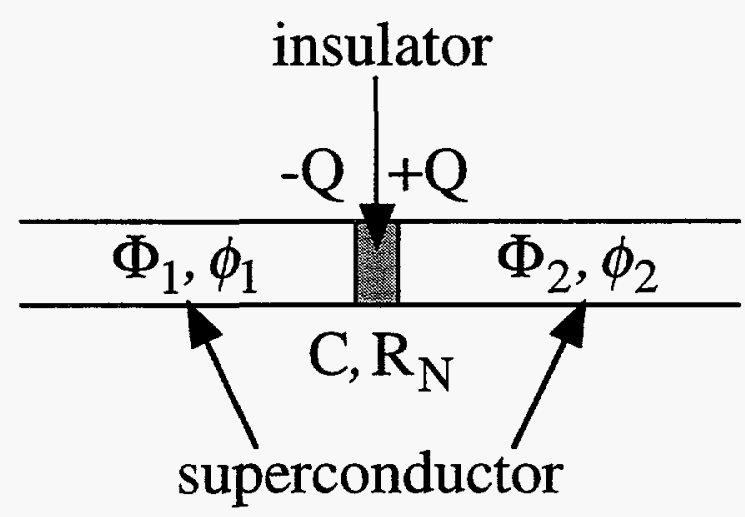

(b)

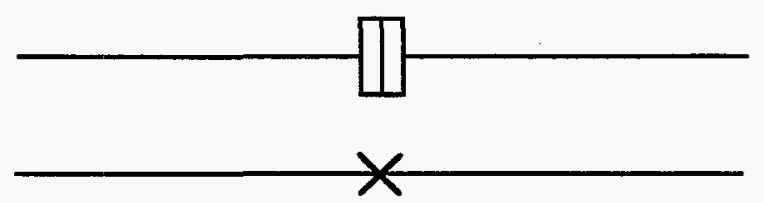

Figure 2.1: Shown in (a) is a schematic drawing of a single Josephson junction with charge $Q$, capacitance $C$, normal state tunneling resistance $R_{Q}$, and phase difference $\phi=\phi_{1}-\phi_{2}$. The superconducting electrodes are described by an order parameter $\Phi_{1,2}=\left|\Phi_{1,2}\right| e^{i \phi_{1,2}}$. Shown in (b) are two commonly used symbols for a Josephson junction. 
$H_{0}$ describes the energy associated with the Josephson junction and the energy stored in the capacitor $C . H_{b}$ is the term representing an external bias source, and $H_{r}$ is the term representing dissipation in the system.

The energy associated with the Josephson junction is $E_{J} \cos \phi$ [48]. $E_{J}$ is the Josephson coupling energy and is given by Ambegaokar and Baratoff [3] by the expression:

$$
E_{J}=\frac{h \Delta}{8 e^{2} R_{N}} \tanh \left(\frac{\Delta}{2 k_{B} T}\right)
$$

where $\Delta$ is the superconducting energy gap of the electrodes, $R_{N}$ is the normal state tunneling resistance of the junction, and $T$ is the temperature. $R_{N}$ is a phenomenological quantity that is defined for the situation when there is a fixed voltage $V$ across the electrodes. The tunneling rate of an electron through the barrier is then proportional to $V$ and is given by $V / e R_{N}$ [23]. In this dissertation, we study junctions in the low-temperature limit, where $\tanh \left(\Delta / 2 k_{B} T\right) \approx 1$, such that $E_{J} \approx h \Delta / 8 e^{2} R_{N}$. The energy stored in the capacitor $C$ is given by $Q^{2} / 2 C$. We can rewrite this energy as $E_{C}(Q / e)^{2}$ with $E_{C}=e^{2} / 2 C ; E_{C}$ is called the charging energy. Therefore,

$$
H_{0}=E_{C}\left(\frac{Q}{e}\right)^{2}-E_{J} \cos \phi
$$

The phase $\phi$ and charge $Q$ are conjugate variables, $[\phi, Q]=2 i e$, and therefore they obey the uncertainty relationship:

$$
\Delta \phi \Delta Q \geq 2 e
$$

This relationship tells us that when $\phi$ is well-defined, $Q$ is completely uncertain, and vice versa. When $E_{J} \gg E_{C}$, the well-defined variable is $\phi$. In the opposite limit, $E_{J} \ll E_{C}$, $Q$ is the well-defined variable.

If the junction is current-biased, and if the current $I$ is constant in time, then the part of the Hamiltonian due to the external source is given by [83]:

$$
H_{b}=-\frac{\hbar I}{2 e} \phi
$$

The problem of how to quantify the dissipation term $H_{r}$ is more difficult. We will not discuss dissipation in single junctions here. ${ }^{1}$

At low temperatures, the thermal energy is small compared to the Josephson energy and charging energy, $E_{J}, E_{C} \gg k_{B} T$. In this limit, thermal effects are not important,

\footnotetext{
${ }^{1}$ For more information about dissipation in single junctions, see Iansati et al. (1988) [43] and Caldeira and Leggett (1981) [14].
} 
and effects due to the charging energy and/or the Josephson energy are dominant. This is the regime studied in this dissertation.

\subsection{Single Superconducting Tunnel Junction}

For a single Josephson junction with $E_{J} \gg E_{C}$, the Hamiltonian can be solved using the resistively-and-capacitively-shunted-junction (RCSJ) model [79,62]. In this case, $\phi$ is taken as the well-defined variable, and $Q$ is written in terms of $\phi$ using the ac Josephson equation [48] $(V=\hbar \dot{\phi} / 2 e)$ and $Q=C V$. In the RCSJ model, the junction is modeled as three branches and a current bias. One of the branches is the "Josephson" branch that carries a supercurrent. The other two branches are a resistance and capacitance that shunt the junction. An inductance for the Josephson branch can be calculated, and thus the junction behaves similarly to an RCL circuit, showing small oscillations, and damping of these oscillations inversely proportional to $R_{d}$, where $R_{d}$ is the resistance that characterizes the dissipation term $H_{r}$ in the Hamiltonian.

The Hamiltonian can be modeled by the "washboard" model, where the junction behavior is likened to that of a particle in a tilted washboard potential [44]. The phase $\phi$ is like the position of the particle; $Q$ is like the momentum of the particle; $C$ is like the mass of the particle. The larger the current bias $I$, the larger the tilt of the washboard potential. The current-voltage characteristics of the junction can be calculated, giving the expected features of a critical current and retrapping current.

\subsection{Single Normal Metal Tunnel Junction}

Now we consider a junction in the limit $E_{J} \ll E_{C}$. In this limit, $Q$ is the welldefined variable. For simplicity, we consider a junction with normal metal electrodes (NIN junction), so $E_{J}=0$. Consider a single NIN junction biased by a current $I$ whose voltage is measured by a high impedance voltmeter. For the moment, we ignore the effects of the environment on the junction. Since the electrodes are normal, the junction is characterized only by its capacitance $C$ and tunneling resistance $R_{N}$. There is a charge $Q$ associated with the junction capacitance. Let us assume that the normal state tunneling resistance of the junction $R_{N}$ is much larger than the quantum of resistance for single electrons, $R_{N} \gg R_{Q e} \equiv h / e^{2} \approx 25.8 \mathrm{k} \Omega$. This condition ensures that the wavefunction of an excess 
electron on an island is localized there [23]. If $R_{N}$ is small compared to $R_{Q e}$, there will be delocalized states where the electrons can flow through an island without charging it, and the interesting "charging" effects discussed in the next paragraph will not be seen.

When a tunneling event occurs, the charge $Q$ must change by a charge $e$. The change in the electrostatic energy of the junction resulting from the tunneling is given by [23]:

$$
\Delta E=\frac{Q^{2}}{2 C}-\frac{(Q-e)^{2}}{2 C}=\frac{e(Q-e / 2)}{C} .
$$

The tunneling event will only occur if it is energetically favorable. Thus, at $T=0$, tunneling will only occur if $\Delta E>0$. This condition gives the result that there will be no current flow in the junction for $\Delta E<0$ :

$$
-\frac{e}{2 C}<V<\frac{e}{2 C}, \quad I=0
$$

This is called the Coulomb blockade.

When there is a non-zero current in the junction, $Q$ will increase linearly until it reaches $\mathrm{e} / 2$. A tunneling event will then occur, and the charge will jump to $-\mathrm{e} / 2$. This gives oscillations in the junction voltage called single electron tunneling (SET) oscillations. The frequency of these oscillations is $f=I / e$. 


\section{Chapter 3}

\section{One-Dimensional and \\ Two-Dimensional Arrays of}

\section{Normal Metal Tunnel Junctions}

\subsection{Introduction}

This chapter discusses the theory of $1 \mathrm{D}$ and $2 \mathrm{D}$ arrays of normal metal tunnel junctions over a ground plane. In Sec. 3.2 and Sec. 3.3, the theory of $1 \mathrm{D}$ and $2 \mathrm{D}$ arrays of normal metal tunnel junctions, respectively, with no offset charges on the islands is discussed, with a description of the Gibbs free energy, statics, and dynamics of the arrays. In Sec. 3.4 is discussed the theoretical work of Middleton and Wingreen [63] on 1D and 2D arrays of normal metal tunnel junctions with offset charges on the islands.

\subsection{One-Dimensional Arrays of Normal Metal Junctions}

In this section is summarized the theory of $1 \mathrm{D}$ arrays of normal metal islands separated by tunnel junctions, with no offset charges on the islands, over a ground plane. Shown schematically in Fig. 3.1 is a $1 \mathrm{D}$ array of $N$ junctions separated by tunnel junctions over a ground plane. The tunnel junctions have a capacitance $C$ and normal state tunneling resistance $R_{N}$. Each island has a capacitance $C_{g}$ to a nearby ground plane. There is a voltage $V$ across the array and a common mode front gate voltage (voltage between the 


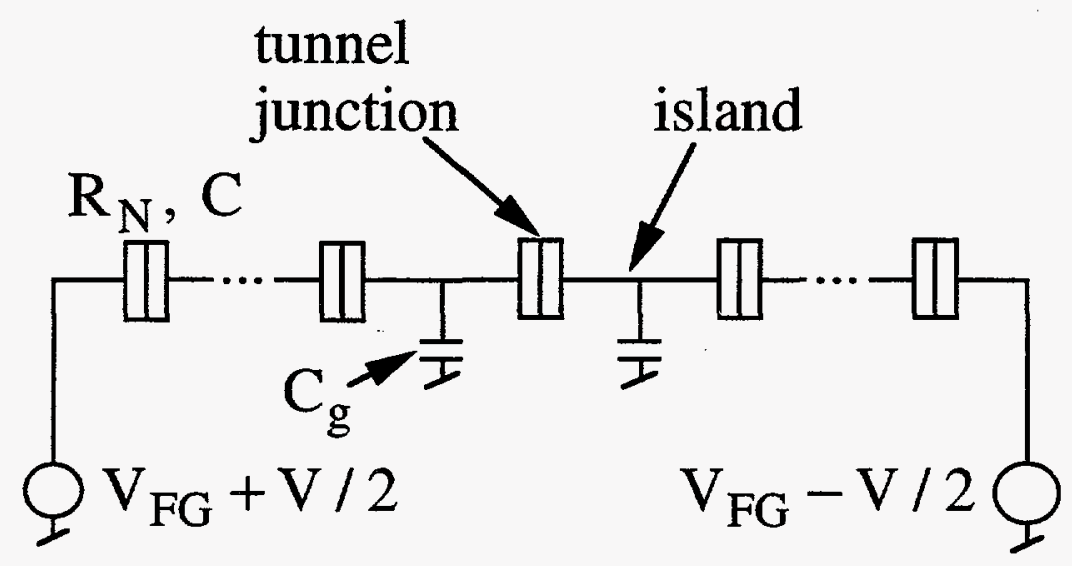

Figure 3.1: Schematic diagram of a 1D array of tunnel junctions over a ground plane. The junctions have capacitance $C$ and normal state tunneling resistance $R_{N}$. The islands have a capacitance $C_{g}$ to ground. There is a voltage $V$ across the array and a front gate voltage $V_{F G}$ between the islands and ground.

array and ground plane) $V_{F G}$. Here we study arrays in the limit of small capacitances $e^{2} /\left[2 \max \left(C, C_{g}\right)\right] \gg k_{B} T\left(E_{J}=0\right.$ since the islands of the array are normal) and large normal state tunneling resistance $R_{N} \gg R_{Q e} \approx 25.8 \mathrm{k} \Omega$.

\subsubsection{Gibbs Free Energy and Tunneling Probability}

In the limit given above of small capacitance and large resistance junctions, an approximation can be used to show that the probability of an individual tunneling act per unit time is given by the difference between the Gibbs free energies of the system before and after the event $[4,6]$. Therefore, to analyze the behavior of a $1 \mathrm{D}$ array, all we need to know is the Gibbs free energy that describes the electrostatic free energy and its interaction with the external voltage source. The electrostatic free energy $\mathcal{F}$ of the $1 \mathrm{D}$ arrays is given by $[4,6]$ :

$$
\mathcal{F}=\frac{C}{2} \sum_{i=0}^{N-1}\left(\Phi_{i+1}-\Phi_{i}\right)^{2}+\frac{C_{g}}{2} \sum_{i=1}^{N-1} \Phi_{i}^{2},
$$

where $\Phi_{i}$ are the potentials of the islands with respect to ground, and $V_{L, R}$ are the voltages of the left and right leads to the array. The potentials of the leads are given by:

$$
\Phi_{0}=V_{L}=\frac{V}{2}+V_{F G}, \quad \Phi_{N}=V_{R}=-\frac{V}{2}+V_{F G} .
$$


The Gibbs energy $\mathcal{G}$ is given by:

$$
\mathcal{G}=\mathcal{F}-Q_{L} V_{L}-Q_{R} V_{R}
$$

where $Q_{L, R}$ are the total charges flowing through the external sources at voltages $V_{L, R}$ :

$$
Q_{L}=C\left(\Phi_{0}-\Phi_{1}\right)+e n_{1}, \quad Q_{R}=C\left(\Phi_{N}-\Phi_{N-1}\right)-e n_{N}
$$

where $n_{i}$ is the number of electrons that have tunneled through junction $i$. Charge conservation gives the potentials $\Phi_{i}$ in terms of the electric charges $Q_{i}=\left(Q_{0}\right)_{i}+e\left(n_{i}-n_{i+1}\right)$ of the islands:

$$
-C \Phi_{i-1}+\left(2 C+C_{g}\right) \Phi_{i}-C \Phi_{i+1}=Q_{i}, \quad i=1, \cdots, N-1
$$

$\left(Q_{0}\right)_{i}$ is the offset charge on island $i$, which depends on the locations of the charged impurities in the insulating layer of the substrate. This offset charge, in real experiments, is a source of disorder in the arrays. Experiments show that the diffusion of these impurities causes $\left(Q_{0}\right)_{i}$ to relax to values $\left|\left(Q_{0}\right)_{i}\right| \ll e[6]$.

For simplicity, we consider first the case $\left(Q_{0}\right)_{i}=0 .{ }^{1}$ Then, for $Q_{j}= \pm \delta_{j, k}$, the system described by Eq. 3.5 can have solutions of the form [4]:

$$
\begin{gathered}
\Phi_{i}^{ \pm}= \pm \frac{e}{C_{e f f}} e^{-\lambda|i-k|}, \quad C_{e f f} \equiv\left(C_{g}^{2}+4 C C_{g}\right)^{1 / 2}, \\
\lambda \equiv \operatorname{arccosh}\left(1+\frac{C_{g}}{2 C}\right) \rightarrow \begin{cases}\ln \left(C_{g} / C\right), & \text { for } C \ll C_{g} \\
\left(C_{g} / C\right)^{1 / 2}, & \text { for } C_{g} \ll C .\end{cases}
\end{gathered}
$$

This solution is valid far away from the edges of the array and $\lambda N \gg 1$. The solution $\Phi_{i}^{+}$ is called a "soliton", and the negative solution $\Phi_{i}^{-}$is called an "antisoliton". A soliton is interpreted physically in the following way: if a charge is placed on an island far away from the edges, then it will polarize nearby islands, with the polarization falling off as $1 / \lambda$. If $1 / \lambda \gg 1$, the array is said to have a "long screening length", and if $1 / \lambda \ll 1$, the array has a "short screening length". In this dissertation, we study arrays with a short screening length $\left(C_{g} \gg C\right)$.

Solitons repulse other solitons, and antisolitons repulse antisolitons. A soliton and antisoliton are attracted to each other; there can be an annihilation of a soliton-antisoliton

\footnotetext{
${ }^{1}$ Unfortunately, the approximation $\left(Q_{0}\right)_{i}=0$ is not an experimentally realistic approximation. Arrays of metallic islands always have offset charges on their islands due to charge impurities in the substrate. Alan Middleton and Ned Wingreen [63] have studied arrays of metallic islands separated by tunnel junctions taking into account offset charges, and in the limit. $C_{g} \gg C$. This theory will be discussed in Sec. 3.4.
} 
pair. A soliton is attracted to an unbiased edge of an array. A soliton is repelled from an edge if the edge has a positive polarity.

\subsubsection{Statics - Threshold Voltage}

If a voltage is applied to the edge of a long array $(N \gg 1 / \lambda)$, the electric field will penetrate a distance $1 / \lambda$ into the array. If the voltage is large enough, an electron will tunnel onto the first island (a soliton is "injected" into the array). The voltage between the array and the ground plane needed to inject a soliton into the array, the threshold voltage $V_{T}$, is given by [6]:

$$
V_{T}=\frac{e}{2 C}(\exp (\lambda)-1)^{-1}
$$

The voltage across the array $V$ required to start a current flowing through the array is different when the array is symmetrically biased and asymmetrically biased. Conduction through the array occurs when the voltage of the edge of the array is raised a voltage $V_{T}$ above the front gate voltage $V_{F G}$. If the array is symmetrically biased, $V_{L}=V_{F G}-V / 2$ and $V_{R}=V_{F G}+V / 2$, and conduction occurs when $V>2 V_{T}$. If the array is asymmetrically biased, $V_{L}=V_{F G}$ and $V_{R}=V_{F G}+V$, and conduction occurs when $V>V_{T}$. Therefore, the threshold for conduction is twice as large for a symmetrically biased array than for an asymmetrically biased array.

\subsubsection{Dynamics - Offset Voltage}

As the voltage across the array is increased above the threshold voltage $V_{T}$, the current $I$ through the array rises above zero. As the voltage is further increased, the current-voltage characteristics of the array tend toward the linear asymptotic form [20]:

$$
I=\frac{1}{N R_{N}}\left(V-V_{o f f}\right)
$$

where $V_{\text {off }}$ is the offset voltage given for a long array $\left(N \gg 1, \lambda_{0}\right.$, where $\left.\lambda_{0}=-\ln \left[1+\frac{C_{g}}{2 C}-\left[\left(\frac{C_{g}}{2 C}\right)^{2}+\frac{C_{g}}{C}\right]^{1 / 2}\right]\right)$ by $[7]$ :

$$
V_{\text {off }}=\frac{e}{C} \times \begin{cases}\frac{N}{2}\left(1-\frac{\lambda_{0}}{2}\right), & \text { for } C_{g} \ll C \\ (N-1) \frac{C}{C_{g}+3 C}, & \text { for } C_{g} \gg C\end{cases}
$$




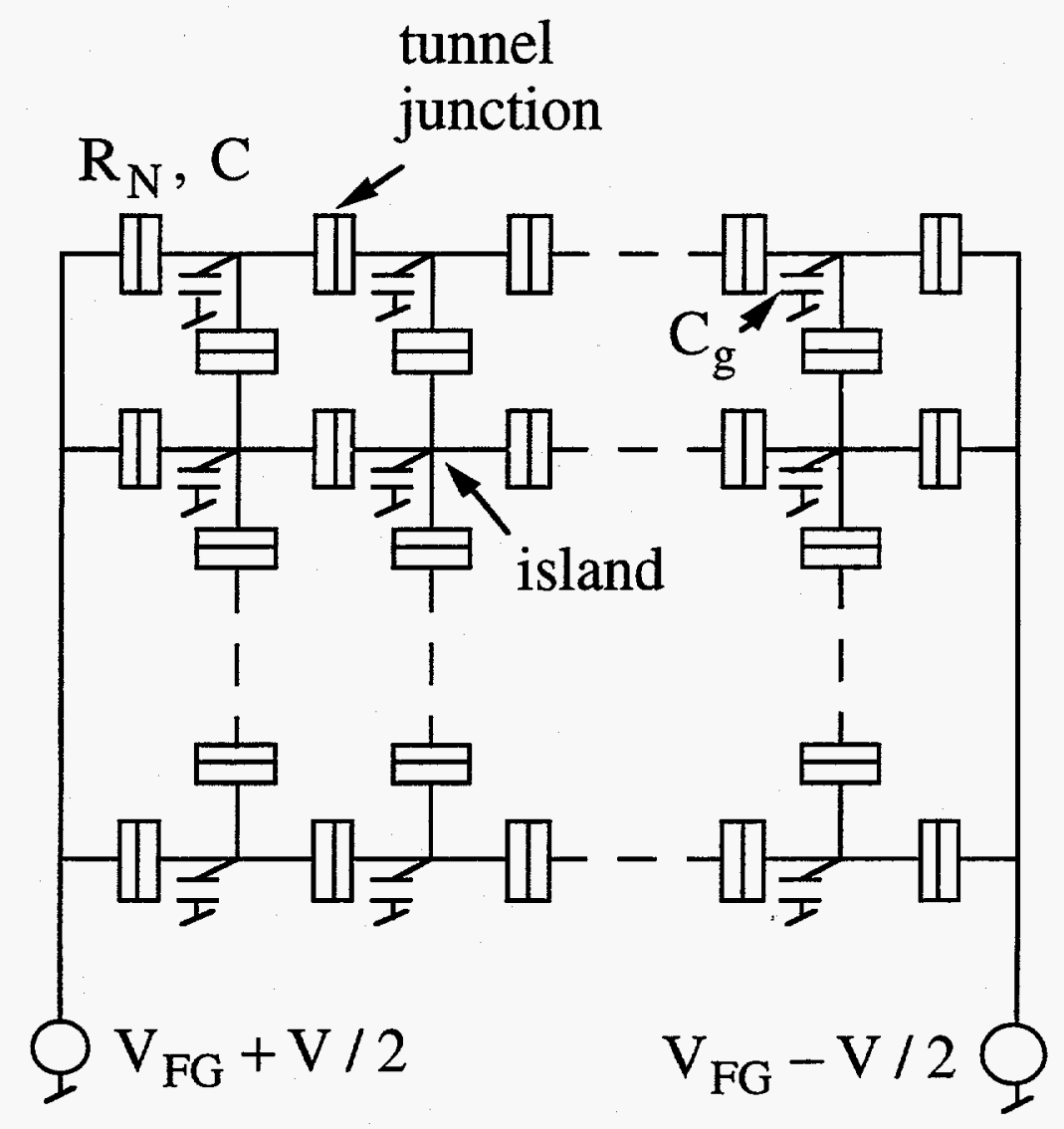

Figure 3.2: Schematic diagram of a 2D array of tunnel junctions over a ground plane. The junctions have capacitance $C$ and normal state tunneling resistance $R_{N}$. The islands have a capacitance $C_{g}$ to ground. There is a voltage $V$ across the array and a front gate voltage $V_{F G}$ between the islands and ground.

\subsection{Two-Dimensional Arrays of Normal Metal Junctions}

In this section the theory of $2 \mathrm{D}$ arrays of normal metal islands separated by tunnel junctions over a ground plane, with no offset charges on the islands, is summarized (ID and $2 \mathrm{D}$ arrays with offset charges are discussed in Sec. 3.4). The summary follows closely the work of Bakhvalov et al. [7]. Shown schematically in Fig. 3.2 is a $2 \mathrm{D}$ array of islands separated by tunnel junctions $((N-1) \times M$ islands $)$ over a ground plane. The tunnel junctions have a capacitance $C$ and normal state tunneling resistance $R_{N}$. Each island has a capacitance $C_{g}$ to a nearby ground plane. There is a voltage $V$ across the array and a common mode front gate voltage $V_{F G}$. 


\subsubsection{Gibbs Free Energy and Tunneling Probability}

As in the case of the $1 \mathrm{D}$ array of normal metal junctions (see Sec. 3.2.1), the 2D array is described by the Gibbs free energy $\mathcal{G}$ as follows:

$$
\begin{aligned}
\mathcal{G} & =\sum_{n=1}^{N-1} \sum_{m=1}^{M} C_{g} \frac{\Phi_{n, m}^{2}}{2} \\
& +\sum_{n=1}^{N} \sum_{m=1}^{M} \frac{C}{2}\left(\Phi_{n, m}-\Phi_{n-1, m}\right)^{2} \\
& +\sum_{n=1}^{N-1} \sum_{m=1}^{M-1} \frac{C}{2}\left(\Phi_{n, m}-\Phi_{n, m+1}\right)^{2} \\
& -\sum_{m=1}^{M}\left(V_{L} q_{L, m}+V_{R} q_{R, m}\right) .
\end{aligned}
$$

Here, $Q_{n, m}$ and $\Phi_{n, m}$ are the electric charges and potentials of the islands, and

$$
q_{L, m} \equiv e k_{L, m}+C\left(V_{L}-\Phi_{L, m}\right), \quad q_{R, m} \equiv e k_{R, m}+C\left(V_{R}-\Phi_{R, m}\right)
$$

where $k_{L, m}\left(k_{R, m}\right)$ are numbers of electrons injected into the islands of the left(right) row from the edge electrodes. Continuity of charge yields:

$$
\begin{array}{r}
-C\left[\Phi_{n-1, m}+\Phi_{n+1, m}+\left(1-\delta_{m, 1}\right) \Phi_{n, m-1}+\left(1-\delta_{m, M}\right) \Phi_{n, m+1}\right] \\
+\left[C_{g}+C\left(4-\delta_{m, 1}-\delta_{m, M}\right)\right] \Phi_{n, m}=Q_{n, m} \\
\text { for } 1 \leq n \leq N-1, \quad 1 \leq m \leq M
\end{array}
$$

where $\Phi_{0, m} \equiv V_{L}, \Phi_{N, m} \equiv V_{R}$.

For large enough tunneling resistance $R_{N} \gg R_{Q e}$, the single electron tunneling rate $\Gamma$ through a junction can be calculated from [4]:

$$
\Gamma=\frac{1}{e R_{N}} F\left(-\frac{\Delta \mathcal{G}}{e}\right), \quad F(x) \equiv \frac{x}{\left[1-\exp \left(-e x / k_{B} T\right)\right]}
$$

where $\Delta \mathcal{G}$ is the change of the Gibbs free energy as the result of a tunneling event. Analytical solutions of Eq. 3.13 can be found [7]. A specific solution will be discussed in the following section. 


\subsubsection{Statics - Two-Dimensional Solitons}

For a large array $(M, N \gg 1)$, an electron placed on an island far away from the edges, $Q_{n, m}= \pm e \delta_{n, n_{0}} \delta_{m, m_{0}}$, will produce a potential distribution:

$$
\Phi= \begin{cases}\frac{ \pm e}{2 \pi C} \ln \left(\frac{1}{r}\right), & \text { for } 1 \ll r \ll \lambda_{0}^{-1} \\ \frac{ \pm e}{2 \sqrt{\pi C C_{e f f}}} \frac{\exp \left(-\lambda_{0}\right)}{\sqrt{r}}, & \text { for } \lambda_{0}^{-1} \ll r\end{cases}
$$

where $r$ is the distance from the charge, $V_{R}$ and $V_{L}$ are the voltages on the right and left sides of the array $\left(V=V_{R}-V_{L}\right)$, respectively, $C_{e f f}=\sqrt{C_{g}^{2}+4 C C_{g}}$, and $\lambda_{0}$ is defined in Sec. 3.2.3. This potential is a two-dimensional single-electron soliton that forms as a result of the charge polarizing nearby islands.

The energy $E$ of the soliton is related to the electric potential $\Phi$ in the relationship $E=e \Phi_{0} / 2$, where $\Phi_{0}$ is the potential at the charge. In the asymptotic limits of the ratio $C_{g} / C, E$ is given by:

$$
E=\frac{e^{2}}{2 C_{g}} \times \begin{cases}\frac{C_{g}}{4 \pi C} \ln \left(\frac{32 C}{C_{g}}\right), & \text { for } C_{g} \ll C \\ 1, & \text { for } C_{g} \gg C\end{cases}
$$

The threshold voltage $V_{T}$, the voltage between an array edge and ground plane necessary to inject solitons into the array, can also be calculated. For a large array $(N, M \gg$ $\left.1, \lambda_{0}^{-1}\right):$

$$
V_{T}=\frac{e}{2 C_{g}} \times \begin{cases}\left(1-\frac{2}{\pi}\right) \times\left(\frac{C_{g}}{C}\right)^{1 / 2}, & \text { for } C_{g} \ll C \\ 1, & \text { for } C_{g} \gg C\end{cases}
$$

\subsubsection{Dynamics - Offset Voltage}

The dynamics of a 2D array cannot be calculated analytically, but can be calculated numerically. Bakhvalov et al. [7] use a Monte Carlo method involving the Gibbs free energy of the whole array (the so-called global rule ${ }^{2}$ ) to calculate the dynamics. As the voltage across the array is increased above the threshold for conduction, the current rises above zero, and the current-voltage characteristics gradually approach the linear asymptote:

$$
I=\frac{M}{N} \frac{1}{R_{N}}\left(V-V_{o f f}\right),
$$

\footnotetext{
${ }^{2}$ Using the "global rule", the energetics of the electron tunneling depend on all the capacitances of the array. Using the "local rule", the energetics of the electron tunneling depend only on the capacitance of the tunnel junction across which it tunnels.
} 
where the offset voltage $V_{\text {off }}$ is given:

$$
V_{o f f}=\frac{e}{C} \times \begin{cases}\frac{N}{4}-\frac{1}{4 \sqrt{2}}, & \text { for } m=M / 2 \text { and } C_{g} \ll C \\ \frac{N}{\pi}+\frac{1}{2}-\frac{1}{\sqrt{2}}, & \text { for } m=1, M \text { and } C_{g} \ll C \\ (N-1) \frac{C}{C_{e f f}}, & \text { for all } m \text { and } C_{g} \gg C,\end{cases}
$$

where $m$ is the row number. This dependence of $V_{o f f}$ on the row number means that even in the limit $V \rightarrow \infty$, the current is not distributed uniformly across the array width, and is slightly lower in the edge rows $(m \approx 1, M)$ due to the soliton repulsion of the edges.

\subsection{One-Dimensional and Two-Dimensional Arrays of Nor- mal Metal Islands with Offset Charges}

In real arrays of normal metal islands separated by tunnel junctions, disorder is always present in the form of charge offsets on the islands due to charge impurities in the substrate. A realistic theoretical investigation of the behavior of these arrays should therefore include these offset charges. Alan Middleton and Ned Wingreen [63] study the behavior of $1 \mathrm{D}$ and $2 \mathrm{D}$ arrays of normal metal islands separated by tunnel junctions, including random offset charges, in the limit of a large number of islands, temperature $T=0$, and small screening length, $C / C_{g} \ll 0$.

\subsubsection{Dynamic Critical Phenomena}

Systems of interacting electrons in the presence of disorder have been studied both theoretically and experimentally, but are still not well understood. Examples of such systems are superconductor- and metal-to-insulator transitions and dynamic critical phenomena, which is displayed in weakly-disordered systems such as sliding charge density waves $^{3}$ and flux line lattices [8]. The dynamics of these weakly-disordered systems have been thoroughly studied, but the dynamics of systems with strong disorder are not so well understood. Middleton and Wingreen propose that transport in arrays of normal metal islands linked by tunnel junctions may be a model system for studying transport in the strong disorder limit.

Dynamic critical phenomena is defined as critical phenomena involving explicit time dependence. Consider transport in the presence of disorder; consider a large number

\footnotetext{
${ }^{3}$ For a review, see [39].
} 
of interacting objects in a random static potential. The velocity $v$ of the objects in the presence of an applied force $F$ is given by the following expressions [30]:

$$
v= \begin{cases}0, & F<F_{T} \\ \left(F / F_{T}-1\right)^{\zeta}, & F>F_{T},\end{cases}
$$

where $F_{T}$ is the threshold force. Below the threshold force $F_{T}$ the objects are pinned and the velocity $v$ of the objects is zero. Above the threshold force, the velocity follows a power law in the reduced force $f=F / F_{T}-1$ with exponent $\zeta$.

Middleton and Wingreen show that arrays of normal metal islands linked by tunnel junctions over a ground plane exhibit dynamic critical phenomena, where the particles are the excess electrons on the islands, the force on the particles is the voltage across the array, and the velocity of the particles is the current through the array. Secs. 3.4.2 through 3.4.5 summarize the work of Middleton and Wingreen on $1 \mathrm{D}$ and 2D arrays of capacitively coupled normal metal islands with offset charges.

\subsubsection{Electrostatic Energy}

Consider an array of normal metal islands capacitively coupled to each other, with charges allowed to tunnel between neighboring islands. For arrays with normal state tunneling resistance much greater than the resistance quantum for single electrons, $R_{N} \gg$ $R_{Q e} \equiv h / e^{2} \approx 25.8 \mathrm{k} \Omega$, the state of the array is fully described by the number of electrons on each island. The energy of the array is then entirely electrostatic and is determined by the capacitance matrix $C_{i j}$. As usual, $C$ is the junction capacitance, and $C_{g}$ is the capacitance to ground of the islands. The electrodes leading to the array and the ground plane are assumed to have infinite self capacitances. The array is assumed to be in the Coulomb blockade regime, where the thermal energy is much smaller than the charging energies associated with the array, $k_{B} T \ll e^{2} /\left[2 \max \left(C, C_{g}\right)\right]$. The electrostatic energy of the system is then given by:

$$
E=\frac{1}{2} \sum_{i, j}\left(Q_{i}+q_{i}\right) C_{i j}^{-1}\left(Q_{i}+q_{i}\right)+V_{L} Q_{L}+V_{R} Q_{R}+\sum_{i} V_{i}^{e x t} Q_{i},
$$

where the sum is over islands $(i, j), Q_{i}$ is the charge on island $i$, and $Q_{L, R}$ are the charges on the left and right leads at voltages $V_{L, R}$. Disorder is included through the offset charges $q_{i}$, where $q_{i}$ represents the effective charge on each island due to nearby trapped charges. Since large fluctuations in disorder are compensated by an integral number of mobile charges, 
$0 \leq q_{i}<e . V_{i}^{e x t}$ is the voltage on island $i$ due to the leads $L, R$ and the gate $g$ and is given by:

$$
V_{i}^{e x t}=\sum_{x} C_{x} \sum_{j}^{x} C_{i j}^{-1} V_{x},
$$

where $\sum_{j}^{x}$ is the sum over islands $j$ that are next to the leads and ground plane, $x=L, R, g$.

At low temperatures, charge may tunnel between dots if the tunneling event lowers the electrostatic energy of the array. Numerical analysis of the tunneling rates in the system show that for large arrays, there is a second order transition with critical phenomena. There is a transition from a static, nonconducting state to a dynamic, conducting state. The parameter that controls this transition is the voltage across the array $V$. For small $V$, charge enters the array and penetrates some distance determined by the random potential of the offset charges; the array then relaxes to a static configuration. For large $V$, the charges are able to travel from one end of the array to the other. At some threshold voltage $V_{T}$, conduction through the array begins.

\subsubsection{Threshold Voltage}

Numerical analysis gives the threshold voltage for conduction:

$$
\lim _{n \rightarrow \infty} \overline{V_{T}}(N) \frac{C_{g}}{N e}=\alpha\left(\frac{C}{C_{g}}\right),
$$

where $\overline{V_{T}}$ is an average of the threshold voltage over disorder, $N$ is the number of junctions in series, ${ }^{4}$ and $\alpha\left(C / C_{g}\right)$ is a function of $C / C_{g}$. Shown in Fig. 3.3 is a schematic plot of $\alpha$ versus the ratio $C / C_{g}$ for $1 \mathrm{D}$ and $2 \mathrm{D}$ arrays. The threshold voltage increases linearly with the linear size of the array. Since $\alpha\left(C / C_{g}\right)$ decreases with increasing $C / C_{g}, \overline{V_{T}}$ also decreases with increasing $C / C_{g}$.

\subsubsection{Interface Motion}

To get an idea of the approach used in determining the threshold voltage in a $2 \mathrm{D}$ array, consider the motion of an interface created by the advancement of charges in a. $2 \mathrm{D}$ array. Fix the voltage of the right lead $V_{R}$ and raise the left lead voltage $V_{L}$. Charge will move from the left lead towards the right lead. The condition for the charge to overcome

\footnotetext{
${ }^{4}$ For a 1D array, $N$ is the number of junctions. For a $2 \mathrm{D}$ array, $N$ is the width of the array, the number of junctions between the bus bars of the array.
} 


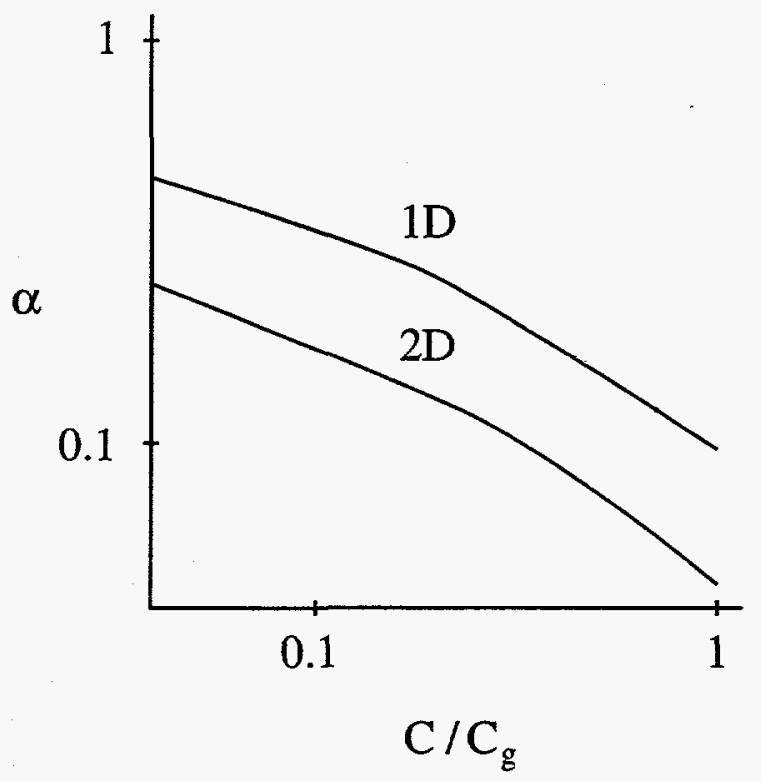

Figure 3.3: Schematic diagram of the function $\alpha$ versus the ratio $C / C_{g}$ from Middleton and Wingreen [63].

the Coulomb barrier and tunnel from island $i$ to island $j$ is given by $V_{i}>V_{j}+e / C_{g}$. For a given $V_{L}$, the advancement of charge stops when $V_{i} \leq V_{j}+e / C_{g}$ everywhere. The particular configuration of charges on the islands at any $V_{L}<V_{T}$ is determined by the disorder realization. A unique interface is defined by the distance the charges penetrate into the array. Conduction occurs when the interface reaches the right edge of the array.

This motion of the interface is similar to stochastic growth of interfaces in models without quenched spatial disorder. Middleton and Wingreen use the results of the KardarParisi-Zhang (KPZ) equation [50] for a $(d-1)$-dimensional interface subject to short-range correlated noise to help in analyzing the behavior of a $d$-dimensional array of normal metal islands.

\subsubsection{Current-Voltage Characteristics}

Analysis performed by Middleton and Wingreen predicts the $I-V$ characteristics of the $1 \mathrm{D}$ and $2 \mathrm{D}$ arrays of normal islands separated by tunnel junctions over a ground plane. For voltage across the array $V$ less than the threshold voltage, $V<V_{T}$, the current through the array $I=0$. For $V \geq V_{T}$, for the reduced voltage $v \equiv\left(V / V_{T}\right)-1, I$ is given 
by:

$$
\begin{aligned}
& \text { For } v \text { small } I \sim v^{\zeta}, \quad \zeta= \begin{cases}1 & \text { for } 1 \mathrm{D} \\
5 / 3 & \text { for } 2 \mathrm{D}\end{cases} \\
& \text { For } v \text { large } \quad I \sim v .
\end{aligned}
$$

Numerical analysis gives $\zeta=1,2.0 \pm 0.2$ for $1 \mathrm{D}$ and $2 \mathrm{D}$ arrays, respectively. Qualitatively, the predicted behavior of the $I-V$ characteristic is as follows: below some threshold voltage, there will be no current through the array; above and near threshold, the current will be nonlinear, being proportional to the power of a reduced voltage; far from threshold, the current will be linear in the reduced voltage. Our experimental work on $1 \mathrm{D}$ and $2 \mathrm{D}$ arrays of normal metal islands linked by tunnel junctions over a ground plane in the limit $R_{N} \gg R_{Q e}$ and $C_{g} \gg C$, with a comparison of our results to the theoretical predictions of Middleton and Wingreen, is described in Chap. 9. 


\section{Chapter 4}

\section{One-Dimensional and Two-Dimensional Arrays of Josephson Junctions}

\subsection{Introduction}

This chapter discusses the theory of $1 \mathrm{D}$ and $2 \mathrm{D}$ arrays of superconducting tunnel junctions (Josephson junctions) over a ground plane. In Sec. 4.2, the theory of $1 \mathrm{D}$ arrays of Josephson junctions is discussed, including a description of the array Hamiltonian and the effects of quantum fluctuations and dissipation on the arrays. In Sec. 4.3 , the theory of $2 \mathrm{D}$ arrays of Josephson junctions is discussed, with a description of the array Hamiltonian and effects of a magnetic field applied perpendicularly to the array. An extensive discussion of superconductor-insulator transitions in $2 \mathrm{D}$ arrays of Josephson junctions is given in Chap. 5 .

\subsection{One-Dimensional Arrays of Josephson Junctions}

Consider a 1D array of superconducting islands separated by Josephson junctions over a ground plane as shown schematically in Fig. 3.1. The junctions have a normal state tunneling resistance $R_{N}$ and capacitance $C$. Each island has a capacitance $C_{g}$ to a nearby ground plane. There is a voltage $V$ across the array and a common mode front gate voltage $V_{F G}$. 


\subsubsection{Hamiltonian}

The Hamiltonian for an isolated 1D Josephson junction array is given by:

$$
H(\phi, Q)=H_{0}+H_{r}
$$

where $H_{0}$ (in the limit in which only nearest-neighbor Coulomb interactions are considered) is given by:

$$
H_{0}=\sum_{i}\left[E_{C}\left(\frac{Q_{i}}{e}\right)^{2}-E_{J} \cos \left(\phi_{i}-\phi_{i+1}\right)\right] .
$$

The sum $i$ is over nearest-neighbor islands in the array, $Q_{i}$ is the excess charge across the junction connecting islands $i$ and $i+1$, and $\phi_{i}$ is the phase of the $i^{\text {th }}$ island. Following the convention used in defining the charging energy for a single electron transistor [5], we define the charging energy for a $1 D$ array as $E_{C} \equiv e^{2} / 2 C_{\Sigma}$ where $C_{\Sigma}$ is the effective capacitance seen by an island in an infinite $1 \mathrm{D}$ array $C_{\Sigma}=\left(C_{g}^{2}+4 C C_{g}\right)^{1 / 2}$ (see Chap. 13). $E_{J}$ is the Josephson energy of a junction, as given in Chap. 2. $H_{r}$ represents the effects of dissipation in the system. Dissipation in $1 \mathrm{D}$ Josephson junction arrays is discussed in Sec. 4.2.3.

\subsubsection{Quantum Fluctuations in One-Dimensional Arrays of Josephson Junctions}

Bradley and Doniach [10] study the effect of quantum fluctuations of the phase in $1 \mathrm{D}$ chains of Josephson junctions. They investigate the behavior of the arrays in two regimes, $C=0, C_{g}>0$ and $C>0, C_{g}=0$. The remainder of this section summarizes the results of their work.

\section{Self-Charging Model (Zero Junction Capacitance)}

In the limiting case $C=0, C_{g}>0$, Bradley and Doniach map the problem of the 1D chain of Josephson junctions onto a $(1+1)$-dimensional (one space and one time dimension) classical XY model. In this case, the inverse temperature plays the role of the size of the system in the time direction, and the charging energy plays the role of the temperature of the classical problem. They find a crossover from phase to charge order as the system passes through the Kosterlitz-Thouless ${ }^{1}$ phase transition, and also find the conduction response changing from superconducting to insulating.

\footnotetext{
${ }^{1}$ The Kosterlitz-Thouless transition will not be discussed in this dissertation. For a review of this transition, see Mooij [64].
} 


\section{Nearest-Neighbor Model (Zero Ground Capacitance)}

In the limiting case $C>0, C_{g}=0$, Bradley and Doniach model the charging energy for a single junction in the chain as the parallel-plate capacitor energy $Q^{2} / 8 C$, where $Q$ is the charge difference across the junction and $C$ is its capacitance. They find the surprising result that there is no phase with quasi-long-range phase coherence. At any nonzero value of $C$, the quantum fluctuations produce exponential decay of the order parameter correlation function at $T=0$. Charge order always prevails over phase coherence, and the conduction response is insulating. Thus, a $1 \mathrm{D}$ array of Josephson junctions with $C>0, C_{g}=0$ should never display superconducting behavior.

\subsubsection{Dissipation in One-Dimensional Josephson Junction Arrays}

Bobbert et al. [9] investigate the $T=0$ phase transitions in a $1 \mathrm{D}$ Josephson junction chain, taking into account quantum fluctuations due to charging energy and the effects of ohmic dissipation. The dissipation in the $1 \mathrm{D}$ array is modeled by an ohmic shunt resistor $R_{s}$, and they assume the quasiparticle contribution can be disregarded. They map the action of the Josephson chain onto the statistical mechanics problem of a charged gas in a $(1+1)$-dimensional space-time dual lattice, in the limit of large ratio of Josephson energy to charging energy $E_{J} / E_{C}$.

The results of Bobbert et al. are summarized in a phase diagram in the $E_{J} / E_{C}$ versus $1 / R_{s}$ plane. They find the plane is divided into three phases as shown in Fig. 4.1. In the phase labeled " $N$ ", the array is resistive, and in phases " $S_{1}$ " and " $S_{2}$ ", the array is superconducting. In both phases $S_{1}$ and $S_{2}$, the vortices form dipoles. But in $S_{1}$, a local probe will always see a vanishing average voltage, while in $S_{2}$, a local probe observes a finite voltage. The transition from $S_{1}$ to $S_{2}$ is a quadrupole unbinding transition, where the time correlations of the phase-slip processes change, and the properties of the voltage fluctuations should change. Experimentally, one should observe more voltage noise in phase $S_{2}$ than in phase $S_{1}$.

\subsection{Two-Dimensional Arrays of Josephson Junctions}

In this section is discussed $2 \mathrm{D}$ arrays of superconducting islands separated by Josephson junctions over a ground plane as shown schematically in Fig. 3.2. The junctions 


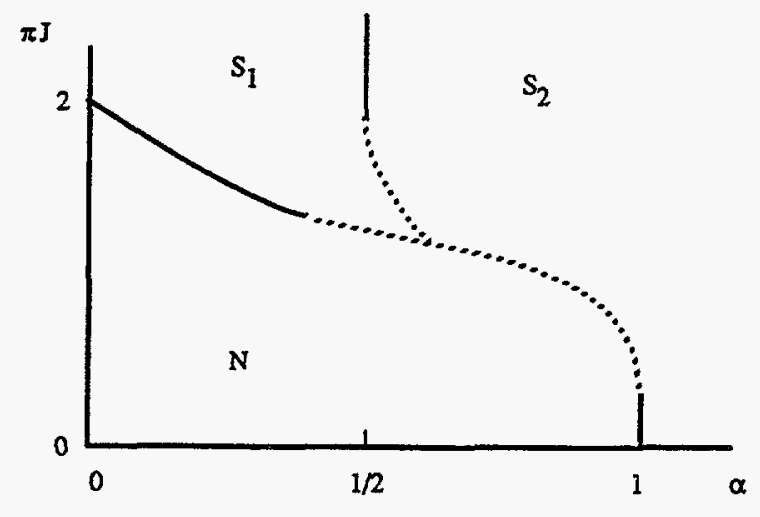

Figure 4.1: From Bobbert et al. [9]; $T=0$ phase diagram in the $J=\left(E_{J} / 8 E_{C}\right)^{1 / 2}$ versus $\alpha=h /\left(4 e^{2} R_{s}\right)$ plane. The $N$ phase refers to the normal state; $S_{1}$ and $S_{2}$ are superconducting states. In $S_{1}$ the dipoles are bound in quadrupoles.

have a capacitance $C$ and normal state tunneling resistance $R_{N}$. Each island has a capacitance $C_{g}$ to a nearby ground plane. There is a voltage $V$ across the array and a common mode front gate voltage $V_{F G}$.

\subsubsection{Hamiltonian}

The Hamiltonian for a 2D array of Josephson junctions over a ground plane is given by:

$$
H(\phi, Q)=H_{0}+H_{r}
$$

where $H_{0}$ (in the limit in which only nearest-neighbor Coulomb interactions are considered) is given by [83]:

$$
\begin{gathered}
H_{0}=\sum_{i, j}\left[E_{C}\left(\frac{Q_{i j}}{e}\right)^{2}-E_{J} \cos \left(\phi_{i}-\phi_{j}-\Psi_{i j}\right)\right], \\
\text { where } \Psi_{i j}=\frac{2 e}{\hbar} \int_{i}^{j} \vec{A} \cdot \overrightarrow{d l} .
\end{gathered}
$$

The sum $(i, j)$ is over nearest-neighbor islands, $Q_{i j}$ is the charge across the junction connecting islands $i$ and $j, \phi_{i}$ is the phase of the $i^{\text {th }}$ island, and $\Psi_{i j}$ is the line integral of the vector potential $\vec{A}$ along a path from island $i$ to $j$. $E_{C}$ is the charging energy, the energy to transfer an electron between neighboring islands, given by $E_{C}=e^{2} / 2 C_{e f f}$, where $C_{e f f}$ is the effective capacitance seen by a charge placed on an island far away from the ends of the array. In the limit $C \gg C_{g}, C_{\text {eff }} \approx C$, and in the limit $C \ll C_{g}, C_{\text {eff }} \approx\left(C_{g}+5 C\right) / 4$ 
(see Chap. 11). $E_{J}$ is the Josephson energy of a junction, given in Chap. 2. $H_{r}$ represents the effects of dissipation in the system. An extensive discussion of dissipation and superconductor-insulator transitions in $2 \mathrm{D}$ Josephson junction arrays is given in Chap. 5 .

\subsubsection{Applied Magnetic Field and Frustration}

When a small magnetic field is applied perpendicularly to a 2D array of Josephson junctions, small units of circulating currents called "vortices" are created in the array (see Appendix C).

A vortex is a special excitation in the configuration of the phases. If one follows a closed contour around the center of a vortex, the sum of all the phase differences is $2 \pi$; the sum of all the phase differences around an antivortex is $-2 \pi$.

The summation of the vector potential contributions around a closed loop containing four junctions should give the applied magnetic flux. Therefore, using the expression for the vector potential in Eq. 4.5:

$$
\sum_{\text {unit cell }} \Psi_{i j}=2 \pi \frac{B a^{2}}{\Phi_{0}}=2 \pi f,
$$

where $B$ is the magnetic field applied perpendicularly to the array, $a$ is the unit cell size, $\Phi_{0}$ is the magnetic flux quantum:

$$
\Phi_{0} \equiv \frac{h}{2 e} \approx 2.07 \times 10^{-15} \mathrm{Tm}^{2}
$$

and $f \equiv B a^{2} / \Phi_{0}$ is called the frustration and is the number of flux quanta per unit cell.

Properties of the 2D array Hamiltonian are invariant under $f \rightarrow f+1$ and $f \rightarrow-f$, so all the major characteristics of the array can be seen in the range $0 \leq f \leq 1 / 2$. For $f$ not close to an integer, the vortices are tightly packed and strongly interacting [75]. For $f$ with the form $p / q$ with $p$ and $q$ small integers (such as $f=1 / 2,1 / 3,3 / 4$ ), the interacting vortex structure is also strongly-coupled to the underlying junction lattice. For small $f$, the vortices act independently, and the only important interaction is with the underlying lattice [75]. The vortex configurations for $f=1 / 2$ and $1 / 3$ are shown in Fig. 4.2. The configuration $f=1 / 2$ is called the "fully frustrated" case.

The "arctan" approximation is an analytical expression that approximates the phase $\phi_{i}$ of an island $i[75]$ :

$$
\phi_{i}=\arctan \left[\frac{y_{i}-y_{0}}{x_{i}-x_{0}}\right]
$$




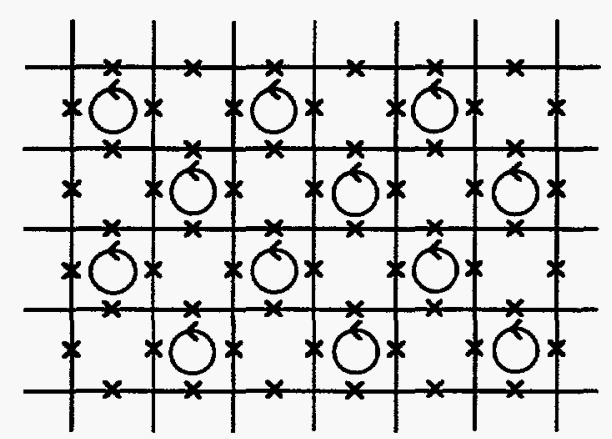

$\mathrm{f}=1 / 2$

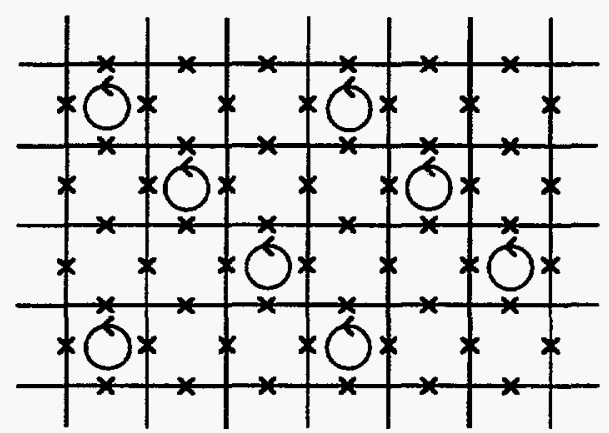

$f=1 / 3$

Figure 4.2: Vortex lattice for frustrations $f=1 / 2$ and $1 / 3$.

where $\left(x_{0}, y_{0}\right)$ gives the position of the center of the vortex and $\left(x_{i}, y_{i}\right)$ is the position of the island $i$.

Numerical calculations of Rzchowski et al. [75] (following the work of Lobb et al. [57]) give the potential energy of a single vortex as a function of the vortex position. The potential is called the "eggcrate" potential, and has minimum energy wells located at the center of the unit cell. There are high energy peaks at the islands and a saddle point on top of the junctions. Therefore, for a vortex to move from one energy well to another, it must have enough energy to go over one of the saddle points. This energy was calculated to be $0.199 E_{J}$ for a square lattice.

External currents will exert a Lorentz force $\overrightarrow{\mathbf{j}} \times \overrightarrow{\mathbf{B}}$ on the vortex, where $\overrightarrow{\mathbf{j}}$ is the sheet current density in the array and $\overrightarrow{\mathbf{B}}$ is the applied magnetic field. Lobb et al. [57] determine the depinning current $I_{d}$ required for a vortex to overcome the energy barrier:

$$
I_{d}=0.199 I_{c} / 2
$$

where $I_{c}$ is the critical current of the junctions. They also determine the free energy of single vortex-antivortex pair:

$$
U_{p r}=E_{\text {core }}+2 \pi E_{J} \ln (r / a)
$$

where $r$ is the vortex-antivortex separation, $a$ is the lattice spacing, and $E_{\text {core }}$ is the free energy of a pair at a separation of one lattice spacing [88] $a, E_{\text {core }}=\pi^{2} E_{J}$. The long-range interaction between the vortex and antivortex in a pair can lead to a Kosterlitz-Thouless transition. 


\subsubsection{Dissipation and Superconductor-Insulator Transitions}

The subject of dissipation and superconductor-insulator transitions in $2 \mathrm{D}$ arrays of Josephson junctions will be discussed extensively in Chap. 5 . 


\section{Chapter 5}

\section{Superconductor-Insulator \\ Transitions in Arrays of Josephson}

\section{Junctions}

\subsection{Introduction}

This chapter discusses the theory of superconductor-to-insulator (S-I) transitions in arrays of Josephson junctions over a ground plane. In Sec. 5.2 is a discussion of the general theory of quantum phase transitions, following closely the discussion in Sondhi et al. [78]. Sec. 5.3 describes S-I transitions in arrays of Josephson junctions driven by a competition between the charging energy $E_{C}$ and the Josephson energy $E_{J}$ of the arrays. Sec. 5.4 discusses S-I transitions driven by a magnetic field applied perpendicularly to the arrays. Sec. 5.5 discusses dissipation-driven S-I transitions, with an emphasis on dissipation due to a nearby ground plane.

\subsection{Quantum Phase Transitions}

Quantum phase transitions are transitions that occur in a quantum system at absolute-zero temperature, $T=0$, as a result of changing some parameter in the Hamiltonian of the system. Unlike classical finite-temperature phase transitions, the dynamic and thermodynamic behavior of quantum phase transitions are intertwined. As the phase 
boundary of the transition is crossed, the quantum ground state of the system changes in some fundamental way. The phase transition occurs not when the temperature is changed, but when some parameter of the Hamiltonian is changed, such as the charging energy in arrays of Josephson junctions or the applied magnetic field in quantum Hall samples. With this definition, all phase transitions that occur at a finite temperature are "classical" [78].

A $d$-dimensional quantum system can be mapped onto a $(d+1)$-dimensional classical system. Consider the partition function of a quantum system and Feynman's pathintegral formulation of quantum mechanics that shows that the net transition amplitude between two states can be calculated by summing amplitudes for all possible paths between them. The expression for the partition function of a $d$-dimensional quantum system looks like a classical partition function for a system with $(d+1)$ dimensions, where the extra dimension is imaginary time. The large body of work on critical behavior of classical systems can thus be used to make general statements about the quantum systems [78].

As an example, consider a 1D Josephson junction array over a ground plane. The Hamiltonian (not considering dissipation) has a term proportional to the Josephson energy $E_{J}$ of the array and a term proportional to the the charging energy $E_{C}$ of the array (see Chap. 4). This $1 \mathrm{D}$ system can be mapped onto the 2D XY model with the Hamiltonian:

$$
H_{X Y}=\frac{1}{K} \sum_{i, j} \cos \left(\phi_{i}-\phi_{j}\right),
$$

where the sum $(i, j)$ is over nearest-neighbor islands, $\phi_{i}$ is the phase of island $i$, and $K$ is a function of $E_{J}$ and $E_{C}, K \sim\left(E_{C} / E_{J}\right)^{1 / 2}$. In the corresponding classical model, $K$ is the temperature. When $K$ is small, $E_{J} \gg E_{C}$, coherent ordering of the phases is favored, and the array is superconducting. When $K$ is large, $E_{C} \gg E_{J}$, zero-point fluctuations of the phase are favored, and the array is insulating. $K$, instead of being a measure of the thermal fluctuations of the system as in a classical system, is a measure of the quantum fluctuations in the system.

Quantum phase transitions are exhibited in $2 \mathrm{D}$ arrays of submicron Josephson junctions over a ground plane at low temperatures. When the fluctuations of the phase of the superconducting order parameter of the system are small, global phase coherence persists, and the array is superconducting. If the phase fluctuations are large, global phase coherence is destroyed, and the array is insulating. A change in a parameter of the Hamiltonian of the array drives the superconductor-to-insulator transitions. Examples of such parameters 
are the charging energy, applied magnetic field, and dissipation. S-I transitions driven by these parameters are described in the next three sections.

\subsection{Charging Energy-Driven Superconductor-Insulator Tran- sition}

In Josephson junctions arrays at low temperatures, there is a competition between two energies, the Josephson coupling energy $E_{J}$ and the charging energy $E_{C} . E_{J}$ is the energy describing the superconducting coupling between islands; $E_{C}$ is the energy describing the tendency of charge carriers to localize. If $E_{J} \gg E_{C}$, the phase of the islands is welldefined and the number of Cooper pairs on an island is undetermined. If $E_{C} \gg E_{J}$, the number of Cooper pairs on an island is well-defined and the phase is undetermined (see Chap. 2).

Suppose we have an array with $E_{J} \geq E_{C}$, such that the array is superconducting at low temperatures. If the charging energy $E_{C}$ of the array can be increased so that $E_{J}<E_{C}$, the array can be driven through a superconductor-to-insulator transition. As the charging energy $E_{C}$ is increased, the quantum fluctuations of the phase increase while the fluctuations of the charge decrease. Therefore the phase coherence of the array is destroyed, and the array makes a transition from superconducting to insulating.

Analytical and numerical studies of the ratio of $E_{C} / E_{J}$ at which this S-I transition should occur in square arrays at $T=0$ in zero applied magnetic field have been performed by various authors [86]. Values of this critical ratio $\left(E_{C} / E_{J}\right)_{c r i t}$ ranging from 1.7 to 10 have been reported $[27,52,47,59]$.

One method of determining $\left(E_{C} / E_{J}\right)_{\text {crit }}$ considers the duality between the dynamics of vortices and Cooper pairs [28]. This calculation is performed in the limit of negligible quasiparticle tunneling and quantum fluctuations and logarithmic interaction between vortex-antivortex pairs and Cooper pair dipoles. ${ }^{1}$ In this and the following two paragraphs, it is assumed that $C / C_{g} \gg 0$ and the charging energy $E_{C} \equiv e^{2} / 2 C$. Then for $E_{J} \gg E_{C}$, there is a vortex unbinding Kosterlitz-Thouless transition at $T_{c}$ from a superconducting to resistive state as $T$ is increased. For $E_{C} \gg E_{J}$, there is a corresponding Cooper pair dipole unbinding transition at $T_{c s}$ from an insulating to resistive state.

\footnotetext{
${ }^{1}$ The interaction between Cooper pair dipoles is logarithmic in the limit $C / C_{g} \gg 0$.
} 
If $E_{J} \approx E_{C}$, then both $T_{c}$ and $T_{c s}$ decrease, and at $\left(E_{J} / E_{C}\right)_{c r i t},{ }^{2}$ both of these critical temperatures vanish, and there is a S-I transition [19]. This "self dual" critical point occurs at $\left(E_{J} / E_{C}\right)_{c r i t, \text { selfdual }}=2 / \pi^{2}[28]$.

Fazio and Schön [28] and others [11,92] show that vortex and charge are not perfectly dual, and they find $\left(E_{J} / E_{C}\right)_{c r i t}=2 a_{c} / \pi^{2}$, where $a_{c} \geq 1$. At $\left(E_{J} / E_{C}\right)_{c r i t}$ the array is neither superconducting nor insulating, so both charge and vortices are mobile. If passing one Cooper pair through the system causes one vortex to cross the system, then it is found that the resistance per square of the system at the critical point should be $R_{s q}=R_{Q}=6.45 \mathrm{k} \Omega$ (for example see [33]).

Experimental studies by van der Zant et al. [86] of square arrays with no ground plane at low temperatures in zero applied magnetic find $\left(E_{C} / E_{J}\right)_{\text {crit }} \approx 1.7$. This study was performed by making many different arrays with different ratios of $E_{C} / E_{J}$ and testing them individually.

\subsection{Magnetic Field-Driven Superconductor-Insulator Tran- sition}

A magnetic field applied perpendicularly to an array of Josephson junctions with $E_{J} \approx E_{C}$ can drive the array through a superconductor-to-insulator transition. Increasing the applied magnetic field $B$ in a superconducting array increases the number of vortices in the array. Above a critical field $B_{c}$, the vortices form a Bose condensate, and the array becomes insulating. The array undergoes a S-I transition from being a vortex glass, where Cooper pairs are Bose condensed and the vortices are localized, to a Bose (or electron) glass, where the vortices are Bose condensed and the Cooper pairs are localized [33].

M. P. A. Fisher [33] developed a scaling theory for this field-tuned S-I transition for disordered superconducting thin films. In particular, a scaling theory for the resistivity of the films near and at the transition is given. Near the transition field $B_{c}$, there is a competition between the condensation of the Cooper pairs and vortices. At low temperatures and low fields (below the bulk transition temperature $T_{c 0}$ and upper critical field $H_{c 2}$ of the film), such a film can be modeled by a system of charge-2e bosons representing Cooper pairs moving in a random potential [31]. Near the transition field, the resistivity $\rho$ of the

\footnotetext{
${ }^{2}$ Some authors prefer to use the ratio $E_{C} / E_{J}$, and others prefer $E_{J} / E_{C}$. We quote directly whichever ratio the author uses.
} 
film should satisfy the scaling form:

$$
\rho(B, T)=\frac{h}{4 e^{2}} \tilde{\rho}\left[\frac{c_{0}\left(B-B_{c}\right)}{1 / T^{-z_{B} \nu_{B}}}\right]
$$

where $c_{0}$ is a nonuniversal constant, $z_{B}$ is the dynamical critical exponent, $\nu_{B}$ is the static critical exponent for the superconducting correlation length $\xi_{B}$, and $\tilde{\rho}$ is a dimensionless scaling function. At the transition, the correlation length $\xi_{B}$ diverges as $\xi_{B} \sim\left(B-B_{c}\right)^{-\nu_{B}}$, and the characteristic frequency of the system $\Omega_{B}$ vanishes as $\Omega_{B} \sim \xi_{B}^{-z_{B}}$. It is found that $\nu_{B} \geq 2 / d$, where $d$ is the dimension (for thin films $d=2$ ), and $z_{B}=1$.

Near the field-driven vortex- to electron-glass transition, Cooper pairs and vortices are playing a dual role. Fisher [33] finds that near the transition, neither the Cooper pairs nor the vortices are condensed. Instead, both are metallic and diffuse with a finite resistance. For logarithmically interacting bosons and Cooper pairs, the transition is selfdual (see previous section) and the sum of the square of the resistivities $\rho_{x x}$ and $\rho_{x y}$ of the film should be given by:

$$
\left(\rho_{x x}\right)^{2}+\left(\rho_{x y}\right)^{2}=R_{Q}^{2}
$$

where $R_{Q}$ is the resistance quantum for Cooper pairs.

The field-driven S-I transition has been observed experimentally in superconducting thin films [41, 81], highly anisotropic three-dimensional superconductors [77], and 2D Josephson junction arrays with no ground plane $[87,18]$. The values of the critical resistance per square $R_{\text {crit }}$ (resistance of the film or array at the critical field $B_{c}$ ) determined from these experiments range from 1.2 to $13.6 \mathrm{k} \Omega$, and the values of the universal scaling exponent product $z_{B} \nu_{B}$ range from 1.2 to 8.2 .

\subsection{Dissipation-Driven Superconductor-Insulator Transition}

When charges or vortices move in an array of junctions, they excite electromagnetic waves. These waves dissipate energy; this dissipation causes damping of the charge or vortex motion. Caldeira and Leggett [14] investigate the influence of dissipation on macroscopic quantum tunneling in macroscopic systems at low temperature. They find that if the interaction of the quantum system with the environment can be described by a phenomenological friction coefficient $\eta$, and assuming the response of the environment is linear, then the dissipation multiplies the tunneling probability by a factor $e^{-A \eta / \hbar}$, where $A$ depends on the parameters of the junction. In short, the dissipation reduces the tunneling probability. 
Let the electrodynamic environment in a superconducting system be characterized by the resistance $R_{e n v}$. Following the work of Caldeira and Leggett, Chakravarty et al. [15] and Fisher [32] find that dissipation in the system causes damping of the phase fluctuations in the superconducting system proportional to $1 / R_{\text {env. }}$. Thus, an increase in the environmental resistance $R_{e n v}$ causes a decrease in dissipation and an increase in phase fluctuations. In a 2D superconducting system such as an array of Josephson junctions, the increase in phase fluctuations destroys global phase coherence and the system becomes insulating.

The main source of dissipation in real single Josephson junctions and arrays of Josephson junctions is unclear. Proposed dissipative mechanisms include shunt resistors across the junctions, quasiparticle tunneling, and a nearby ground plane. These three mechanisms will be discussed in the following sections.

\subsubsection{Shunt Resistors}

Chakravarty et al. [15] investigate the effects of dissipation in arrays of Josephson junction at low temperature. The arrays are modeled as having shunt resistors across the junctions. The effect of dissipation is taken into account by coupling to a heat bath, following the work of Caldeira and Leggett [12, 13]. For arrays with shunt resistors $R_{S}$ across the junctions, they find that dissipation introduces damping of the phase of the superconducting order parameter that is proportional to $1 / R_{S}$. When the damping of the phase fluctuations is small, phase fluctuations are large, long-range phase coherence is destroyed, global superconductivity is destroyed, and the array is resistive. When damping of the phase fluctuations is large, phase fluctuations are small, long-range coherence is restored, and the array is superconducting. They find that the dissipation-driven transition from superconducting to resistive behavior in the array occurs at $R_{Q} / R_{S}=1 / d$ for $d=1,2$ for $1 \mathrm{D}$ and $2 \mathrm{D}$ arrays, respectively.

Fisher [32] also investigates the effects of dissipation due to shunt resistors across the junctions in Josephson junction arrays at $T=0$. In contrast to previous work, a form of the charging energy is chosen that incorporates the long-range Coulomb interaction. Fisher finds that for a $1 \mathrm{D}$ array, the system is always statically disordered, even in the superconducting state. There is a dynamical transition from a superconducting to resistive state at the critical value of the shunt resistor $R_{S, c}=R_{Q}$. Below this critical shunt 
resistance, the array is superconducting; above this critical shunt resistance the array is resistive. For a $2 \mathrm{D}$ array, both the dynamical response and static ordering depend on the the shunt resistance. For $R_{S}<R_{Q}$, dissipation suppresses quantum fluctuations, and the array is superconducting. For $R_{S} \geq R_{Q}$, the order parameter suffers phase slips due to quantum tunneling, and the array is driven normal.

In summary, theoretical investigations of shunt resistors as a dissipative mechanism in $1 \mathrm{D}$ and $2 \mathrm{D}$ Josephson junction arrays show the arrays should undergo a superconductornormal state transition as the value of the shunt resistor $R_{S}$ is increased above the resistance quantum $R_{Q}$. Unfortunately, there is no physical evidence for a shunt resistance across junctions in real arrays.

\subsubsection{Quasiparticle Tunneling}

Various authors consider dissipation in arrays of Josephson junctions due to quasiparticle tunneling. Chakravarty et al. [16] consider the effects of quasiparticle tunneling at energies greater than the energy gap of the superconducting islands. It was shown by Ambegaokar, Eckern, and Schön [2] that quasiparticle tunneling has the effect of renormalizing the capacitance of the junction in a single junction or superconducting quantum interference device (SQUID). Chakravarty et al. extend this idea to $2 \mathrm{D}$ arrays of Josephson junctions and find a critical value of the normal state resistance of the array $R_{N, c}$ that depends weakly on the ratio $\Delta / E_{C}$, where $\Delta$ is the superconducting energy gap of an island, and $E_{C}$ is the charging energy of an island, defined here as $E_{C} \equiv 4 e^{2} / C$. When the normal state resistance $R_{N}<R_{N, c}$, the array is superconducting, and when $R_{N}>R_{N, c}$, the array is insulating. More specifically, when the ratio of $\Delta / E_{C}$ is varied between $10^{-1}$ and $10^{-2}$, the critical resistance $R_{N, c}$ varies between $0.9 R_{Q}$ and $1.4 R_{Q}$.

Kampf and Schön [49] also investigate dissipation in arrays due to quasiparticle tunneling. In contrast to the work by Chakravarty et al., they investigate the effects of quasiparticle tunneling at energies less than the superconducting energy gap of the islands. They concentrate on dissipation associated with a finite subgap conductance $1 / R_{q p}$ ( $R_{q p}$ is the subgap resistance). They find that the dissipation due to this finite subgap conductance shifts the known superconductor-to-insulator transitions (for example, due to a decrease in the ratio of $\left.E_{J} / E_{C}\right)$ to higher critical temperatures or lower critical values of the ratio $E_{J} / E_{C}$ for $1 \mathrm{D}, 2 \mathrm{D}$, and $3 \mathrm{D}$ Josephson junction arrays. 


\subsubsection{Ground Plane}

Recent work by Wagenblast et al. [93], investigates 2D arrays of Josephson junctions over a nearby ground plane, where the resistance of the ground plane can be varied. This work was prompted by our experimental work on the same system, a $2 \mathrm{D}$ array of Josephson junctions capacitively coupled to a two-dimensional electron gas (2DEG), where the resistance per square of the $2 \mathrm{DEG}$ could be varied independently of other parameters of the system (see Chap. 11). Wagenblast et al. find that dissipation due to the ground plane can cause the array to exhibit quasi-critical behavior; the array undergoes a superconductor-to-insulator transition as the ground plane resistance is increased. In this section, the work of Wagenblast et al. is summarized.

Wagenblast et al. model our experimental setup with a Josephson junction array capacitively coupled to a $2 \mathrm{DEG}$. The quantum-dynamical variables describing a Josephson junction array are the phases $\phi$ of the superconducting order parameter. The dynamics of the 2DEG are described by a fluctuating scalar potential $V(\overrightarrow{\mathbf{r}}, t)$ that can be represented by a phase-variable $\theta$, where $\hbar \dot{\theta}=2 \mathrm{eV}$. The action of the coupled system is of the form:

$$
S[\phi, \theta]=S_{\text {array }}[\phi]+S_{2 D E G}[\theta]+S_{I}[\phi, \theta]
$$

where $S_{a r r a y}, S_{2 D E G}$, and $S_{I}$ are the action for the array, 2DEG, and interaction between the array and 2DEG, respectively. $S_{\text {array }}$ contains two terms, one proportional to the Josephson coupling energy $E_{J}$ and one proportional to the junction capacitance $C$, which represents the bare unscreened Coulomb interaction in two dimensions. $S_{I}$ describes the coupling of the array to the $2 \mathrm{DEG}$ and is proportional to the capacitance to ground $C_{g}$ of the islands, which screens the Coulomb interaction. The dynamics of the 2DEG is ohmic, with resistance $R_{2 D E G}$. $S_{2 D E G}$ is proportional to $R_{Q} / R_{2 D E G}$, where $R_{Q}$ is the resistance quantum for Cooper pairs. The microscopic details of the 2DEG do not play a role on the length scales considered here.

The effective action for the array contains the propagator for the $\phi$ field:

$$
D_{0}^{-1}\left(k, \omega_{\mu}\right)=\frac{C}{4 e^{2}} k^{2} \omega_{\mu}^{2}+\frac{C_{g}}{4 e^{2}} \frac{k^{2} \omega_{\mu}^{2}}{k^{2}+\left|\omega_{\mu}\right| / \Omega_{0}}
$$

where $1 / \Omega_{0} \equiv R_{2 D E G} C_{g}$. In the limit of small frequencies $\omega_{\mu}$, the dynamics of the phase is capacitive, with the propagator being given by $D_{0}^{-1}=\omega_{\mu}^{2}\left(C k^{2}+C_{g}\right) /\left(4 e^{2}\right)$. The 2DEG screens the electrodynamic interaction in the array beyond the length scale given by $\sqrt{C / C_{g}}$. 
Dissipative dynamics are frozen out for $\omega_{\mu}<\Omega_{0}$. At frequencies greater than $\Omega_{0}$, the resistivity of the $2 \mathrm{DEG}$ induces damping of the superconducting phase of the array. This damping is caused by the voltage fluctuations of the 2DEG that cannot follow adiabatically the fluctuations of $\phi$. This lag creates a damping with a strength determined by the 2DEG resistance. The propagator is then given by $D_{0}^{-1}=C k^{2} \omega_{\mu}^{2} /\left(4 e^{2}\right)+k^{2}|\omega| R_{Q} /\left(2 \pi R_{2 D E G}\right)$. At higher frequencies $\omega_{\mu} \gg \Omega_{1} \equiv 1 /\left(R_{2 D E G} C\right)$, the dynamics of the phase are again capacitive, but now determined by the capacitance $C$. In summary, the effective action for the array is ohmic, that is, the dynamics of the array are dissipative, only in the frequency range $\Omega_{0}<\omega_{\mu}<\Omega_{1}$. Above and below this frequency range, the dynamics of the phase are capacitive. In the case $C_{g} \gg C$, the characteristic frequencies $\Omega_{0}$ and $\Omega_{1}$ separate the frequency domain into three distinct regimes.

A true quantum phase transition is driven by the behavior of the action at the lowest frequencies (temperatures). Since the dissipative action is cut off at the lowest frequencies, a dissipation-driven transition cannot occur in the strict sense. However, a quasi-critical behavior can be observed at temperatures and voltages exceeding the low energy scale $\Omega_{0}$. The behavior converges to a true dissipation-tuned transition in the limit $\Omega_{0} \rightarrow 0\left(C_{g} \rightarrow \infty\right)$.

The quasi-critical behavior is characterized by evaluating the electromagnetic response of the array at finite temperatures. The regime $E_{J}<E_{C}$, where the charging energy is defined here as $E_{C}=e^{2} / C_{g}$, is investigated. The array conductivity is calculated via the Kubo formula. The conductivity $\sigma(T)$ calculated for the intermediate temperature/frequency range $\Omega_{0}<T<\Omega_{1}$ behaves as $T^{2 / \alpha-2}$, where $\alpha \equiv R_{Q} / R_{2 D E G}$. The predicted resistance of the array $R$ (in units of the resistance quantum $R_{Q}$ ) versus the temperature $T$ is shown in Fig. 5.1. For $\alpha>1, R(T)$ decreases steadily with decreasing $T$, indicating an impending S-I transition. However, since the dissipation is frozen out below $\Omega_{0}, R(T)$ exhibits a sharp rise, eventually becoming an insulator at $T=0$. For $\alpha<1, R(T)$ monotonously increases with decreasing $T$. Wagenblast et al. also calculate the resistivity of the array including thermally activated quasiparticles, by including a parallel thermally activated channel $R_{N} \exp \left(E_{a} / T\right)$, where $E_{a}$ is the activation energy. The predicted resistance of the array $R$ (in units of the resistance quantum $R_{Q}$ ) versus the temperature $T$, including a parallel thermally activated channel, is shown in Fig. 11.4 in Chap. 11.

The results can be interpreted as the renormalization of $E_{J}$ by the dissipative 


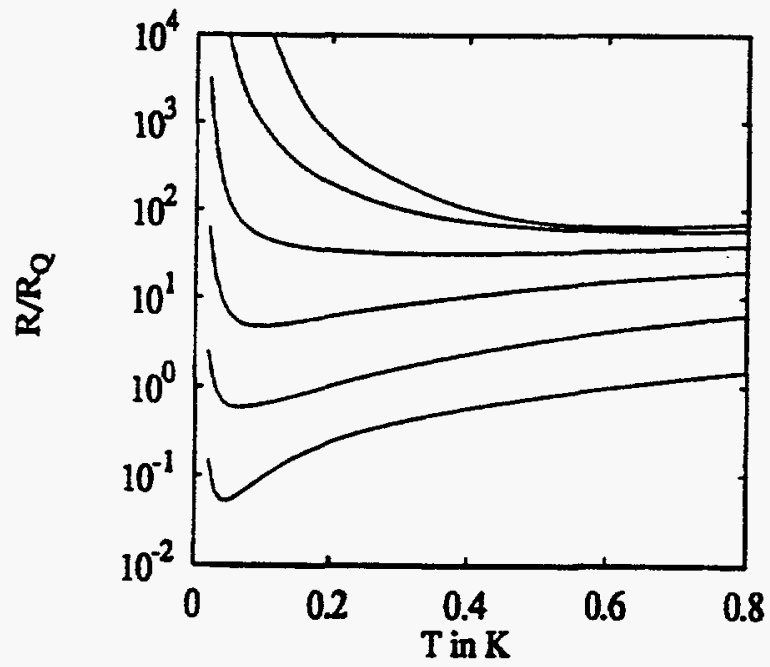

Figure 5.1: Predictions of Wagenblast et al. for the zero-bias resistance of an array, in units of the resistance quantum $R_{Q}$, versus temperature for six different values of the ratio $\alpha=R_{Q} / R_{2 D E G}=50$ (lowest curve), $5,2,1,0.5$, and 0.25 (uppermost curve). The array parameters are $e^{2} / C_{g}=0.3 \mathrm{~K}, E_{J}=0.28 \mathrm{~K}$ and $C_{g} / C=10$.

processes, where the renormalized $E_{J}$ is given by $E_{J}^{r e n} \approx T^{1 / \alpha-1}$. The renormalization stops at $\sim \Omega_{0}$. At $\Omega_{0}$, the model is equivalent to an XY model, with renormalized parameters. This model has a phase transition at $E_{J}^{r e n} / E_{C} \approx 1$. Therefore the S-I phase boundary is given by $E_{J} / E_{C} \approx\left(C / C_{g}\right)^{1-1 / \alpha}$. The predicted S-I phase boundary in the $E_{J} / E_{C}$ versus $\alpha$ plane is shown in Fig. 11.6 in Chap. 11. In the insets, the predicted shape of the array resistance $R$ versus $T$ is shown for the four quadrants. A comparison of the theoretical results of Wagenblast et al. and our experimental results is given in Chap. 11. 


\section{Part II}

\section{Experimental Techniques}


The experimental techniques used to fabricate and measure the arrays of submicron Josephson junctions over a ground plane are described in this part. The fabrication of the samples starts with the preparation of the ground plane, the Si and GaAs/AlGaAs substrates. Both substrates contain a conducting region topped by approximately $100 \mathrm{~nm}$ of an insulator. The Si substrates are made from 4-inch diameter degenerately-doped Si wafers purchased from the Polishing Corporation of America. We carefully grow a thermal oxide layer on the wafers and make ohmic $\mathrm{Al}$ contacts to the conducting $\mathrm{Si}$. The GaAs/AlGaAs substrates are made from pieces of 2-inch diameter GaAs/AlGaAs heterostructures with a two-dimensional electron gas (2DEG) grown by Prof. A. C. Gossard and his group at the University of California at Santa Barbara. We alloy ohmic InSn contacts to the conducting region of the GaAs/AlGaAs substrates. Both the $\mathrm{Si}$ and $\mathrm{GaAs}$ substrates are tested at a temperature of $4.2 \mathrm{~K}$ to verify the contacts to the conducting regions are ohmic. The arrays are then fabricated on the substrates using electron-beam lithography and a shadow evaporation technique [24, 42].

The completed array over its ground plane is then mounted on a copper sample holder specially designed for our dilution refrigerator. The GaAs/AlGaAs substrates with a 2DEG are first placed on a metallic back gate made of undoped $\mathrm{Si}$ with a layer of gold deposited on the surface. Electrical contact to the array and ground plane is made by sandwiching wires between two small In pieces that are pressed to the contact pads to the array and ground plane.

The sample is then tested either at room temperature or at $4.2 \mathrm{~K}$ to measure the average normal state tunneling resistance per junction $R_{N}$. The Si samples are tested at room temperature, and the GaAs samples containing a 2DEG are tested at $4.2 \mathrm{~K}$ since there is leakage between the array and 2DEG through the insulating AlGaAs layer at room temperature. If the array has the average tunneling resistance $R_{N}$ we desire, the sample is put into the dilution refrigerator, and electrical measurements are performed on the sample using both homemade and commercial amplifiers and other electronic equipment. A Macintosh Quadra 650 computer is used to collect the data using the data acquisition program LabView. 


\section{Chapter 6}

\section{Sample Preparation}

\subsection{Substrate Preparation Techniques}

\subsubsection{Silicon Substrates}

The substrates are degenerately-doped ${ }^{1}$ Si topped with approximately $100 \mathrm{~nm}$ of thermal oxide to provide electrical isolation between the array and the conducting Si. The substrates are prepared from $100 \pm 0.5 \mathrm{~mm}$ thick 4-inch Si wafers with $\langle 100\rangle$ orientation, $\mathrm{n}$-doped (with phosphorus) to a resistivity of $\leq 0.008 \Omega-\mathrm{cm}$ (at $300 \mathrm{~K}$ ), and covered with nominally $100 \mathrm{~nm}$ of thermally grown $\mathrm{SiO}_{2}$. These substrates were purchased from the Polishing Corporation of America (Santa Clara, CA). Si was chosen as the substrate material because of the processing expertise and equipment available locally at the Microfabrication Laboratory at the University of California at Berkeley. From each 4-inch wafer we fabricate sixty-nine $1 \mathrm{~cm}^{2}$ substrates with $102 \pm 5 \mathrm{~nm}$ thermally grown $\mathrm{SiO}_{2}$ and four $1 \mathrm{~mm}^{2} \mathrm{Al}$ contact pads to the $\mathrm{Si}$, one at each corner. This preparation is performed at the Microfabrication Laboratory; a detailed outline of the substrate preparation is given in Appendix A. A summary of the Si substrate fabrication procedure is the following:

1. Strip original oxide ${ }^{2}\left(\mathrm{SiO}_{2}\right)$ from wafer and grow $80 \mathrm{~nm}$ thermal oxide.

2. Etch holes for contact pads to $\mathrm{Si}$ in oxide.

3. Grow $55 \mathrm{~nm}$ oxide in contact pad region and increase non-pad oxide region to 100 $n m$.

\footnotetext{
${ }^{1} \mathrm{~A}$ degenerate semiconductor is a semiconductor that is so heavily doped that it is metallic.

${ }^{2}$ We remove the original $100 \mathrm{~nm}$ of $\mathrm{SiO}_{2}$ because we are unsure of its quality and uniformity.
} 


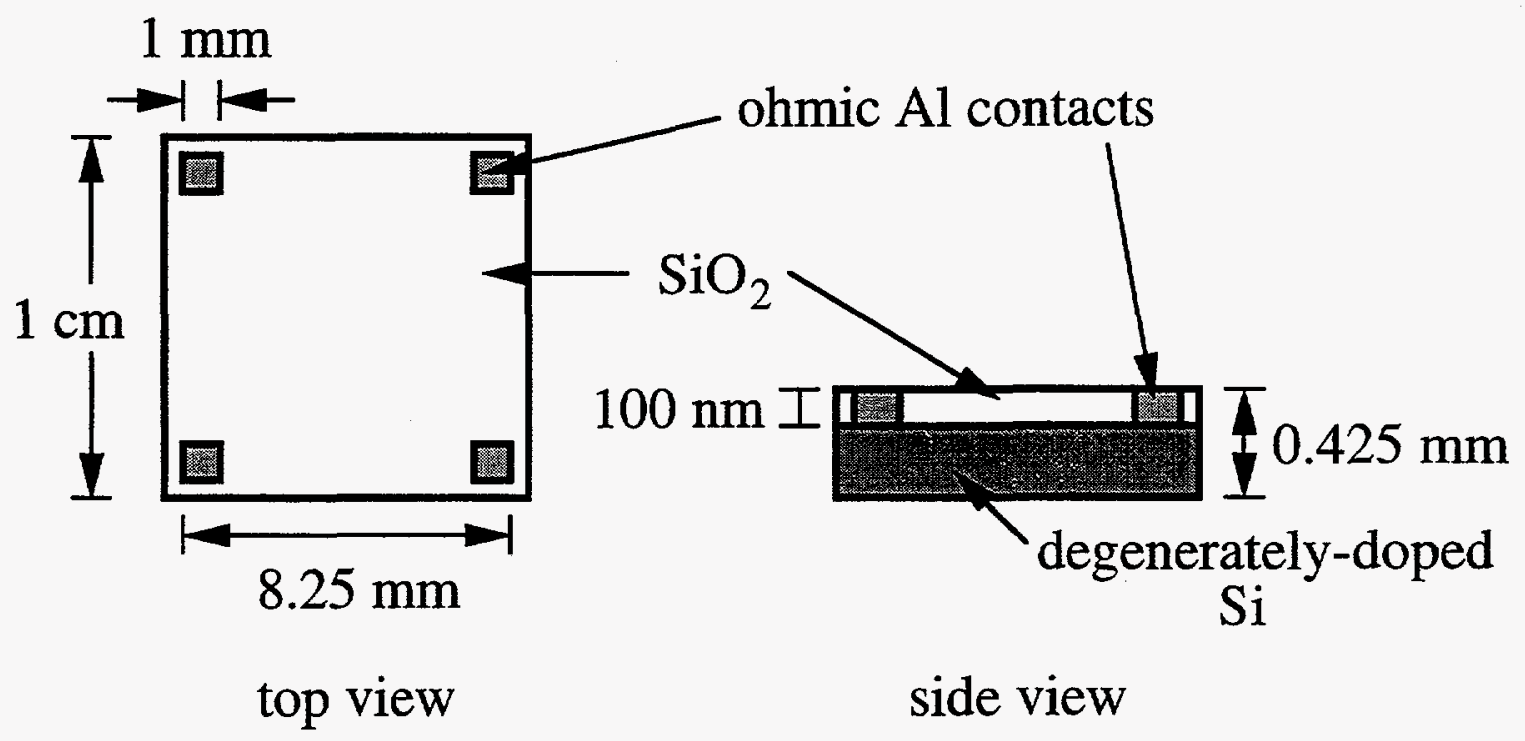

Figure 6.1: Schematic drawing of a degenerately-doped Si substrate with $100 \mathrm{~nm}$ oxide and Al contact pads.

4. Implant and activate arsenic in contact pad region. ${ }^{3}$

5. Sputter and sinter $100 \mathrm{~nm}$ aluminum in contact pad areas.

6. Dice wafer into $1 \mathrm{~cm}^{2}$ substrates and clean substrates.

The end result of the $\mathrm{Si}$ substrate fabrication is shown schematically in Fig. 6.1. The resistivity of one of the $\mathrm{Si}$ substrates was measured down to a temperature of $4.2 \mathrm{~K}$ with a lock-in measurement using a van der Pauw technique [85]. At $239 \mathrm{~K}$ the resistivity of the conducting $\mathrm{Si}$ is $\rho=2.55 \mathrm{~m} \Omega-\mathrm{cm}$ and at $4.2 \mathrm{~K}$ the resistivity drops to $\rho=1.22 \mathrm{~m} \Omega-\mathrm{cm}$.

\subsubsection{GaAs Substrates}

The idea to use GaAs/AlGaAs as a substrate material for the arrays of Josephson junctions originated with Alex Rimberg and Çağllyan Kurdak, two postdoctoral researchers with experience using GaAs heterostructures. They realized that using GaAs/AlGaAs heterostructures as a substrate material would allow us to change the parameters of the ground plane in situ, something which had not been done previously. We use GaAs/AlGaAs heterostructures with a two-dimensional electron gas (2DEG) at the GaAs/AlGaAs interface,

\footnotetext{
${ }^{3}$ Ion implantation of the contact pad region of the $\mathrm{Si}$ is performed to ensure that the contacts to the $\mathrm{Si}$ will be ohmic.
} 


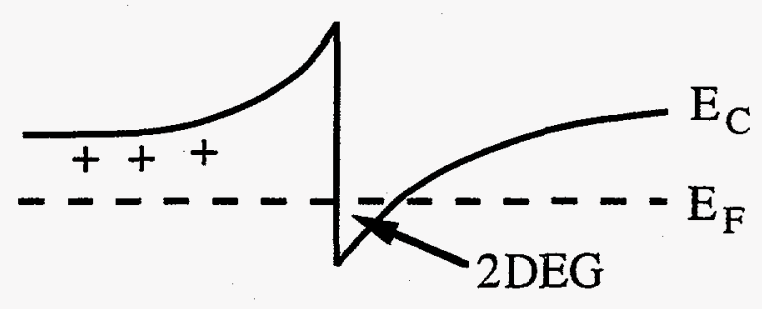

$\mathrm{Al}_{0.3} \mathrm{Ga}_{0.7} \mathrm{As} \quad$ GaAs

Figure 6.2: Band diagram of a 2DEG in a GaAs/AlGaAs heterostructure. $E_{C}$ is the conduction band, and $E_{F}$ is the Fermi level.

where the resistance of 2DEG, the ground plane, can be changed in situ. GaAs/AlAs heterostructures with the GaAs doped such that it behaves as a three-dimensional electron gas (3DEG) are also used by Cağliyan Kurdak. In these heterostructures, the capacitance of the islands in the array to the ground plane can be changed in situ. The samples with GaAs/AlAs substrates will not be discussed in this dissertation.

\section{GaAs/AlGaAs Heterostructures}

The GaAs/AlGaAs heterostructures were grown on undoped GaAs substrates, using molecular beam epitaxy, by K. L. Campman and A. C. Gossard at the University of California at Santa Barbara. Two different heterostructures, one with a lower carrier density than the other, are used. The first heterostructure substrate, "2DEG-A", consists of the following layers: $500 \mathrm{~nm}$ of GaAs, $92 \mathrm{~nm}$ of $\mathrm{Al}_{0.3} \mathrm{Gaa}_{0.7} \mathrm{As}$, and $8 \mathrm{~nm}$ of $\mathrm{GaAs}$. The $\mathrm{Al}_{0.3} \mathrm{Ga}_{0.7} \mathrm{As}$ is selectively doped with $\mathrm{Si}$ donors situated $32 \mathrm{~nm}$ from the lower $\mathrm{GaAs} / \mathrm{Al}_{0.3} \mathrm{Ga}_{0.7} \mathrm{As}$ interface, at which the 2DEG is formed. The second heterostructure, substrate "2DEG-B" consists of the following layers: $500 \mathrm{~nm}$ of GaAs, $104 \mathrm{~nm}$ of $\mathrm{Al}_{0.3} \mathrm{Ga}_{0.7} \mathrm{As}$, and $6 \mathrm{~nm}$ of GaAs. The $\mathrm{Al}_{0.3} \mathrm{Ga}_{0.7} \mathrm{As}$ is selectively doped with Si donors situated $40 \mathrm{~nm}$ from the lower $\mathrm{GaAs} / \mathrm{Al}_{0.3} \mathrm{Ga}_{0.7} \mathrm{As}$ interface.

In such heterostructures, electrons are confined to the GaAs/AlGaAs interface by a potential well that is formed by repulsion arising from a $0.3 \mathrm{~V}$ conduction band offset between the GaAs and AIGaAs and attraction to the positively-charged ionized donors in the $\mathrm{n}$-doped AlGaAs layer (see the band diagram in Fig. 6.2). The substrates are thinned by hand with special thinning tools available at the Microfabrication Laboratory 


\begin{tabular}{|l|l|l|l|}
\hline substrate & $n_{s}\left(\mathrm{~cm}^{-2}\right)$ & $\mu\left(\mathrm{cm}^{2} / \mathrm{Vs}\right)$ & $R_{g}(\Omega / \mathrm{sq})$ \\
\hline \hline 2DEG-A & $2.1 \times 10^{11}$ & $1.8 \times 10^{5}$ & 170 \\
\hline 2DEG-B & $1.5 \times 10^{11}$ & $3.6 \times 10^{5}$ & 120 \\
\hline
\end{tabular}

Table 6.1: Parameters of the 2DEGs in the GaAs/AlGaAs heterostructures at $V_{B G}=0$ and $T=20 \mathrm{mK}$.

to a thickness of $200-250 \mu \mathrm{m}$, and then cleaved into $2 \mathrm{~mm} \times 3 \mathrm{~mm}$ size pieces. ${ }^{4}$ After an array is fabricated on a GaAs heterostructure substrate, the substrate is placed on a metallic back gate. We bias the back gate negatively relative to the $2 \mathrm{DEG}$ with a large voltage $V_{B G}$ to change the sheet density $n_{s}$ of the electrons in the $2 \mathrm{DEG}$, thereby changing its resistance per square $R_{g}$. The substrate is thinned to minimize the back gate voltage $V_{B G}$ needed to change the sheet density $n_{s}$. The sheet density $n_{s}$ of substrates 2DEG-A and $2 \mathrm{DEG}-\mathrm{B}$ at $V_{B G}=0$ and $\mathrm{T}=20 \mathrm{mK}$ are $2.1 \times 10^{11} \mathrm{~cm}^{-2}$ and $1.5 \times 10^{11} \mathrm{~cm}^{-2}$, respectively. The sheet density $n_{s}$, mobility $\mu$, and resistance per square $R_{g}$ of substrates 2DEG-A and 2DEG-B at $V_{B G}=0 \mathrm{~V}$ and $\mathrm{T}=20 \mathrm{mK}$ are given in Table 6.1.

\section{GaAs Heterostructure Contacts}

Ohmic contacts are made to the 2DEG in the GaAs/AlGaAs heterostructures using an InSn alloying technique. The procedure used to make ohmic contacts to the GaAs heterostructures is as follows:

1. Clean substrate by ultrasounding in acetone for $10 \mathrm{~min}$.

2. Solder 4-6 small InSn pieces onto the corners and edges of the substrate with a small soldering iron.

3. Place sample in tape heater oven. ${ }^{5}$

4. Flush oven with forming gas $\left(10 \% \mathrm{H}_{2}, 90 \% \mathrm{He}\right)$ for $1 \mathrm{~min}$.

5. Bake substrate at $120^{\circ} \mathrm{C}$ for $1 \mathrm{~min}$. to evaporate adsorbed water.

\footnotetext{
${ }^{4}$ We make the GaAs substrates smaller than the $\mathrm{Si}$ substrates because we have less GaAs substrate material.

${ }^{5}$ The tape heater oven is a sealed plastic box with a $1 \mathrm{~cm}$ wide strip of $\mathrm{NiCr}$ with electrodes connected to each end that lead out of the walls of the box to a power supply. The box also has an inlet and outlet for gas flow and a thermocouple attached to the underside of the $\mathrm{NiCr}$ strip to measure the temperature.
} 
6. Ramp temperature to $410^{\circ} \mathrm{C}$ and hold at $410-420^{\circ} \mathrm{C}$ for $2-3 \mathrm{~min}$.

7. Ramp temperature down.

8. Stop flow of $\mathrm{H}_{2} / \mathrm{He}$.

Typically the 2DEG contacts are tested at $4.2 \mathrm{~K}$ before fabricating arrays on the substrates.

\subsection{Array Fabrication Techniques}

\subsubsection{Introduction}

The arrays of Josephson junctions studied in this dissertation have submicron features that cannot be made using standard optical lithography. To make $100 \mathrm{~nm}^{2}$ or smaller area Josephson junctions, we use the electron-beam lithography and shadow evaporation technique $[24,42]$. A schematic diagram of this technique is shown in Fig. 6.3. The substrate is first coated with two layers of electron-beam resist, the lower layer having a higher sensitivity than the upper layer. The resist-covered substrate is then exposed by a carefully-controlled electron beam in a scanning electron microscope (SEM). Exposure to an electron beam breaks the bonds in the monomers of the resist, making it soluble in a developer. The exposed substrate is then developed, with more of the higher sensitivity resist being dissolved than the lower sensitivity resist. The resist layers thus form a mask with an undercut, leaving some suspended bridges of resist where the higher sensitivity resist is dissolved, but the lower sensitivity resist remains. Metal is then evaporated onto the substrate through the resist mask from two different angles, such that the metals from the two different evaporations overlap in some regions. An oxide layer is formed on the bottom metal layer by introducing a small amount of oxygen gas into the evaporation chamber between the two evaporations. Metal-oxide-metal junctions are thus formed where the two metal layers overlap. The excess metal evaporated on the parts of the substrate that are coated with resist is then removed with a procedure called "liftoff". During liftoff, the substrate coated with resist and metal is placed in a bath of acetone. The resist is soluble in acetone, and thus the metal-coated resist lifts away, leaving only the metal evaporated directly onto the substrate. 
a) Exposure

electron beam

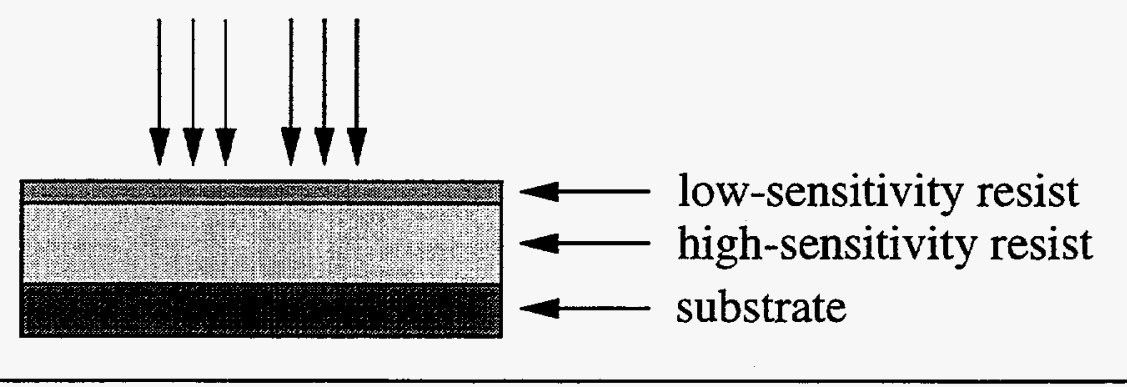

b) Development

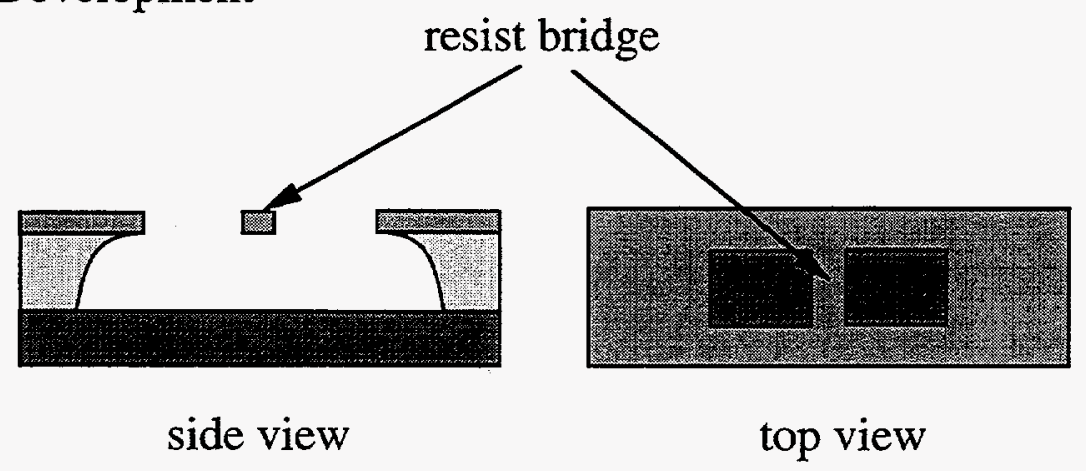

c) Shadow Evaporation

second evaporation $\square \quad$ first evaporation $\square$

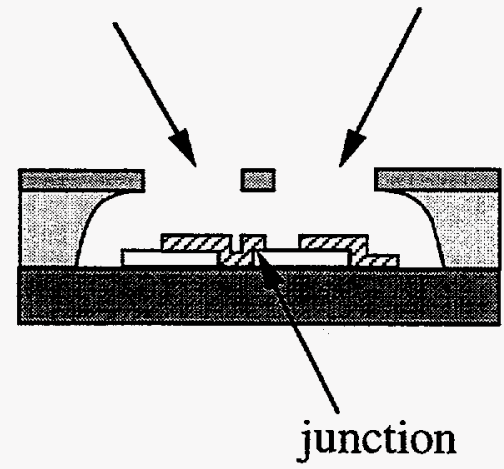

Figure 6.3: The three basic steps of electron-beam lithography and shadow evaporation. 


\subsubsection{Electron-Beam Lithography}

In electron-beam lithography, a substrate is coated with one or more layers of a resist that is sensitive to radiation (electron-beam resist). When the resist is exposed to a beam of high energy electrons, it becomes soluble in a developer solution. ${ }^{6}$ The resist-coated substrate is exposed to a finely-controlled beam of electrons in an SEM. The electron-beam is used like a pencil to draw a pattern in the resist. The exposed substrate is then put into a developer solution, where the exposed parts of the resist wash away, leaving a mask of the pattern drawn with the SEM.

\section{Electron-Beam Resist}

The resist most commonly used in electron-beam lithography is poly(methyl methacrylate) (PMMA), a positive resist. As with other positive resists, exposure to an electron beam has the effect of breaking the bonds of the monomer in the resist molecule. When enough energy is delivered to a volume of resist, it becomes soluble in a developer solution.

To make our arrays of submicron junctions, we used a bilayer resist technique [24, 42]. We coat our substrates with two different layers of electron-beam resist, the lower layer of resist having a higher sensitivity than the upper layer. This technique is used to produce an undercut in the resist profile as shown in Fig. 6.3 (b). The lower layer of resist has a lower molecular weight and is more sensitive to exposure to an electron beam than the higher-molecular-weight upper layer, so that more bonds are broken in the lower layer than the upper layer for the same exposure to an electron beam. Therefore, more of the lower layer of resist will be removed during development, and the resist will have an undercut profile. Such a profile makes liftoff easier by minimizing the possibility that metal on the substrate will be connected to metal on the resist.

For the lower layer of resist, we use the copolymer poly(methyl methacrylate/methacrylic acid) (P(MMA/MAA)), 8.5\% in 2-ethoxyethanol. The $\mathrm{P}$ (MMA/MAA) is spun onto each substrate at $3000 \mathrm{rpm}$ for 30 seconds. The substrates are then placed on a $1 / 8$ inch thick piece of $\mathrm{Al}$ and baked at $150^{\circ} \mathrm{C}$ in a hot-plate oven for 1 hour. The substrates are allowed to cool to room temperature, and then a layer of PMMA, $2 \%$ in chlorobenzene, is spun onto each substrate at $3000 \mathrm{rpm}$ for 30 seconds. The substrates are baked again on the Al plate

\footnotetext{
${ }^{6}$ Resists that are rendered soluble by radiation are called positive resists, while those that are rendered insoluble are called negative resists.
} 
at $150^{\circ} \mathrm{C}$ in a hot-plate oven for 1 hour. Since the GaAs heterostructure substrates are so small $\left(2 \mathrm{~mm} \times 3 \mathrm{~mm}\right.$ ), we first glue them to thin $1 \mathrm{~cm}^{2}$ pieces of copper before spinning on the resist. The GaAs substrates are glued to the copper by spinning P(MMA/MAA) onto the copper pieces at $1000 \mathrm{rpm}$ for 30 seconds, placing the GaAs substrate on the copper near an edge, and then baking the assembly on the Al plate a.t $90^{\circ} \mathrm{C}$ on a hotplate for 10 $\min$.

No direct measurement of the resist layer thicknesses was made by us, but a former member of our group, Andrew Cleland, found that a layer of PMMA (2\% in chlorobenzene) spun on at $3000 \mathrm{rpm}$ for 30 seconds was $100 \mathrm{~nm}$ thick [76]. Thus we believe our upper layer of PMMA is also approximately $100 \mathrm{~nm}$ thick, and the lower layer of P(MMA/MAA) is thicker than $100 \mathrm{~nm}$, since the $\mathrm{P}(\mathrm{MMA} / \mathrm{MAA})$ is denser than the PMMA. The rule of thumb for the bilayer resist technique is that the bottom layer of resist should be roughly three times as thick as the total thickness of metal deposited [83]. Since our first layer of deposited $\mathrm{Al}$ is approximately $20 \mathrm{~nm}$ thick, a bottom layer resist thickness of $100 \mathrm{~nm}$ or greater is sufficient. The top layer of resist merely needs to be thick enough not to sag in the regions of undercut.

After the substrates are coated with resist, they are ready to be used for electronbeam lithography. The substrates are stored in chip trays and kept away from light as much as possible. We find that the resist-coated substrates can be used reliably for lithography months after the resist is applied.

\section{Electron-Beam Exposure Setup}

After the substrates are coated with layers of electron-beam resist, they are exposed to an electron beam in a scanning electron microscope (SEM). The SEM system we use is a JEOL 6400 SEM with the Nabity Pattern Generation System (NPGS) (J. C. Nabity Lithography Systems, Bozeman, MT) located in the Microfabrication Laboratory.

The JEOL 6400 is a state-of-the-art high-resolution SEM. It can provide accelerating voltages from 0.2 to $40 \mathrm{kV}$ and beam currents from $10 \mathrm{pA}$ to $10 \mu \mathrm{A}$. The cathode is a high-brightness lanthanum hexaboride $\left(\mathrm{LaB}_{6}\right)$ filament. The SEM has a Faraday cup for beam current measurements and a fast beam blanker with a rise time of $3 \mu$ s. The stage in the SEM is driven by a computer.

NPGS interfaces to the JEOL 6400 via two 16-bit digital-to-analog converters for 
control of the electron beam position and the beam blanking mechanism. The digital-toanalog converters and other hardware for NPGS are mounted on a printed circuit board that is installed in an IBM PC compatible computer. NPGS interfaces to the user via DesignCAD (American Small Business Machines, Inc., Pryor, OK), a commercial computer-aided design program. A user creates a pattern for beam writing by making a drawing of the pattern in DesignCAD and saving it as a DesignCAD file. Different colors in the pattern correspond to different doses to be used in the exposure. The user then uses the NPGS program MRF (Make Run File) to read in the drawing file and specify doses for the different colors in the drawing and microscope settings such as magnification and beam current. This information is stored in a "run file". An exposure is performed by running the program PG (Pattern Generation). This program accesses the information in the run file to control the position of the electron beam and the beam blanking [73].

\section{Exposure Considerations}

The known quantities in an exposure with an electron beam in an SEM are the beam current density and the exposure time, so that exposures are usually quantified in terms of dose (charge per unit area). The dose actually received by a volume of resist at a given depth below the surface is not simply related to the amount of charge per unit area delivered above it. Mostly because of backscattering of electrons by the substrate, a particular feature is irradiated not only by electrons delivered directly to it, but also to some degree by electrons scattered from nearby features. This phenomenon is called the proximity effect. As a result of this effect, large features require less exposure than small ones because of electrons scattered from another part of the same feature. Similarly, isolated features require more exposure than those close to many other features [73].

\section{Sample Pattern}

The pattern we expose to make our arrays of junctions is shown schematically in

Fig. 6.4. The entire structure consists of three different patterns exposed at three different magnifications. The pattern exposed at the highest magnification is the pattern for the array of islands linked by tunnel junctions. Shown in Fig. 6.5 is an SEM micrograph of a $2 \mathrm{D}$ array with 38 columns and 40 rows of islands. The $2 \mathrm{D}$ arrays are $2 \mathrm{D}$ lattices of islands with a square unit cell. The shape of an island is modeled after the island shape by Tom 


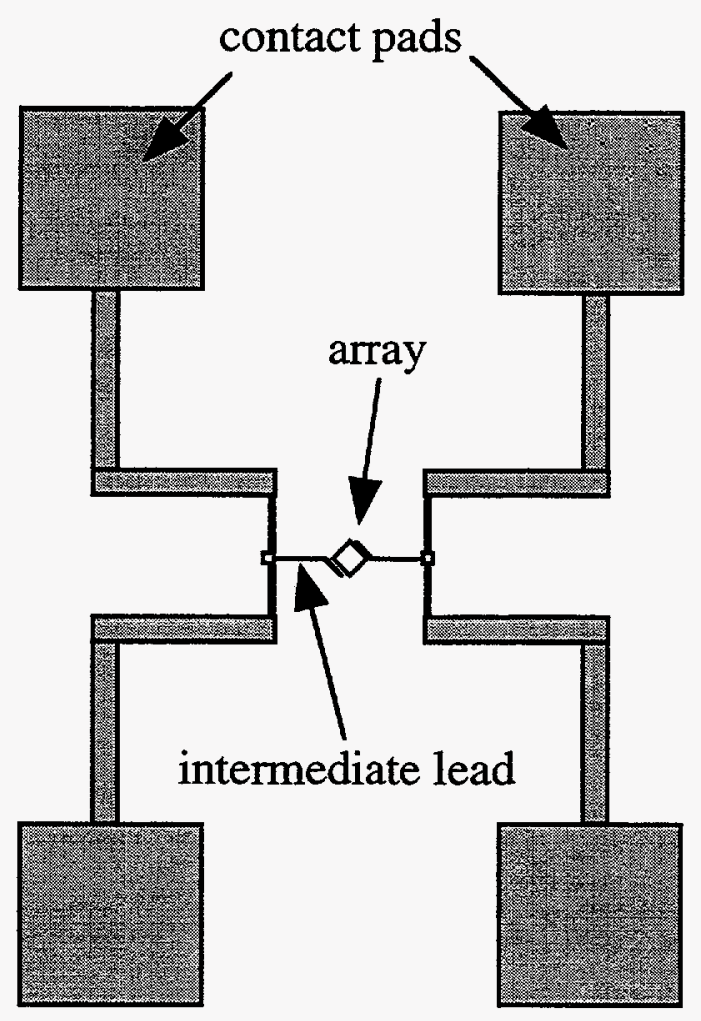

Figure 6.4: Electron-beam lithography sample pattern, including the array, intermediate leads, and contact pads. 


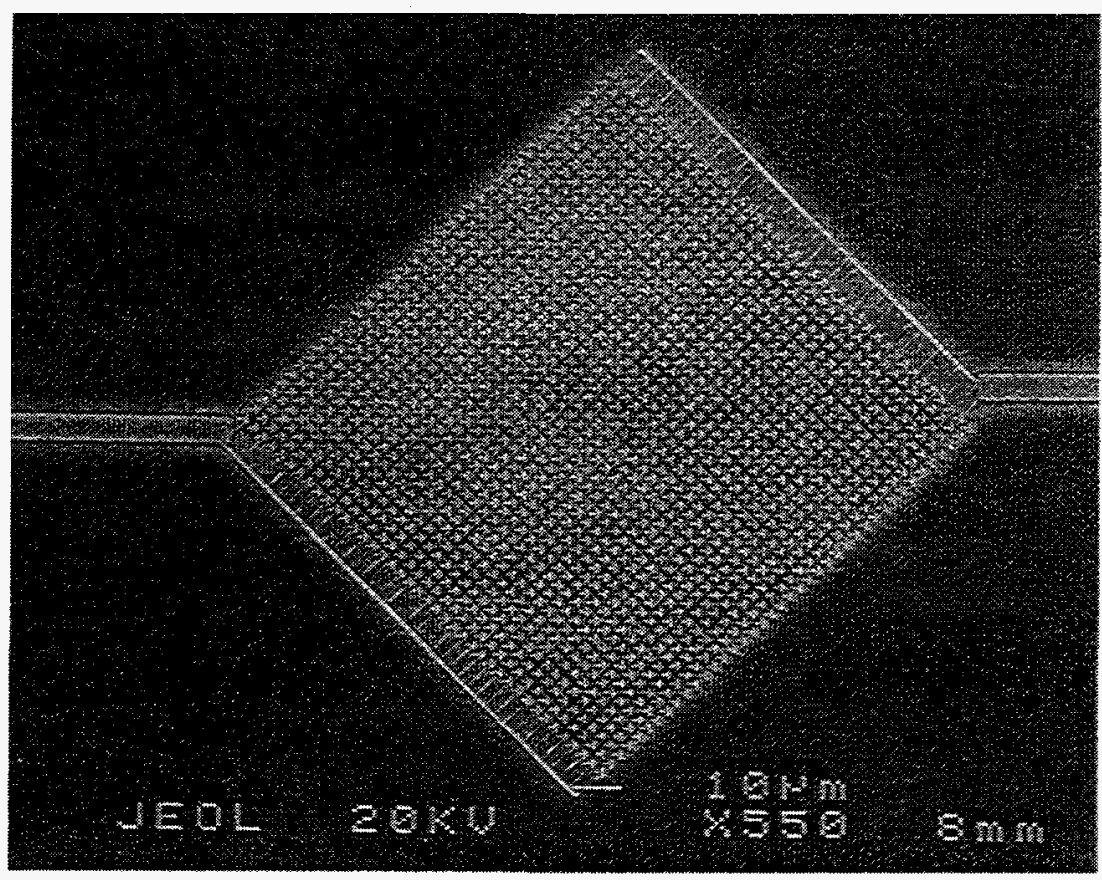

Figure 6.5: SEM micrograph of a 2D array with 38 columns and 40 rows of islands. Each bright spot is an island. Two bus bars connect islands on opposite sides of the array. The bus bars connect to intermediate leads going to contact pads, two on each side of the array.

Tighe [83]. Shown in Fig. 6.6 is an SEM micrograph of an island in a $2 \mathrm{D}$ array.

The junctions are formed where the thin horizontal line from one island overlaps the thin vertical line of its neighboring island after the angled evaporations of metal. An SEM micrograph of a single junction is shown in Fig. 6.7. Every island in the inside of the array is linked to its four nearest neighbors by a junction. We chose the "T" shape of the bulk of the island to maximize the distance between an island and its neighbors, both to minimize the exposure proximity effect between islands and to ensure that the capacitance between islands will be dominated by the capacitance of the junction $C$. The area of the islands was chosen to give a specific capacitance to the ground plane of the islands $C_{g}$. For the island capacitance $C_{g}$ to be approximately the same in the arrays on both the Si and GaAs substrates, we make the island area on the GaAs substrates smaller than on the $\mathrm{Si}$ substrates. $^{7}$

\footnotetext{
${ }^{7}$ The capacitance of parallel plates of area $A$ separated by a distance $d$ filled with a material of dielectric constant $\kappa$ is given by $C=\kappa \epsilon_{0} A / d$, where $\epsilon_{0}$ is the permittivity of free space. The dielectric constant of the insulator in the $\mathrm{Si}$ substrates, $\mathrm{SiO}_{2}$, is 3.9; the dielectric constant of the insulator in the GaAs substrates, $\mathrm{Al}_{0.3} \mathrm{Ga}_{0.7} \mathrm{As}$, is approximately 11 . Therefore, since the insulating layers in both substrates are approximately
} 


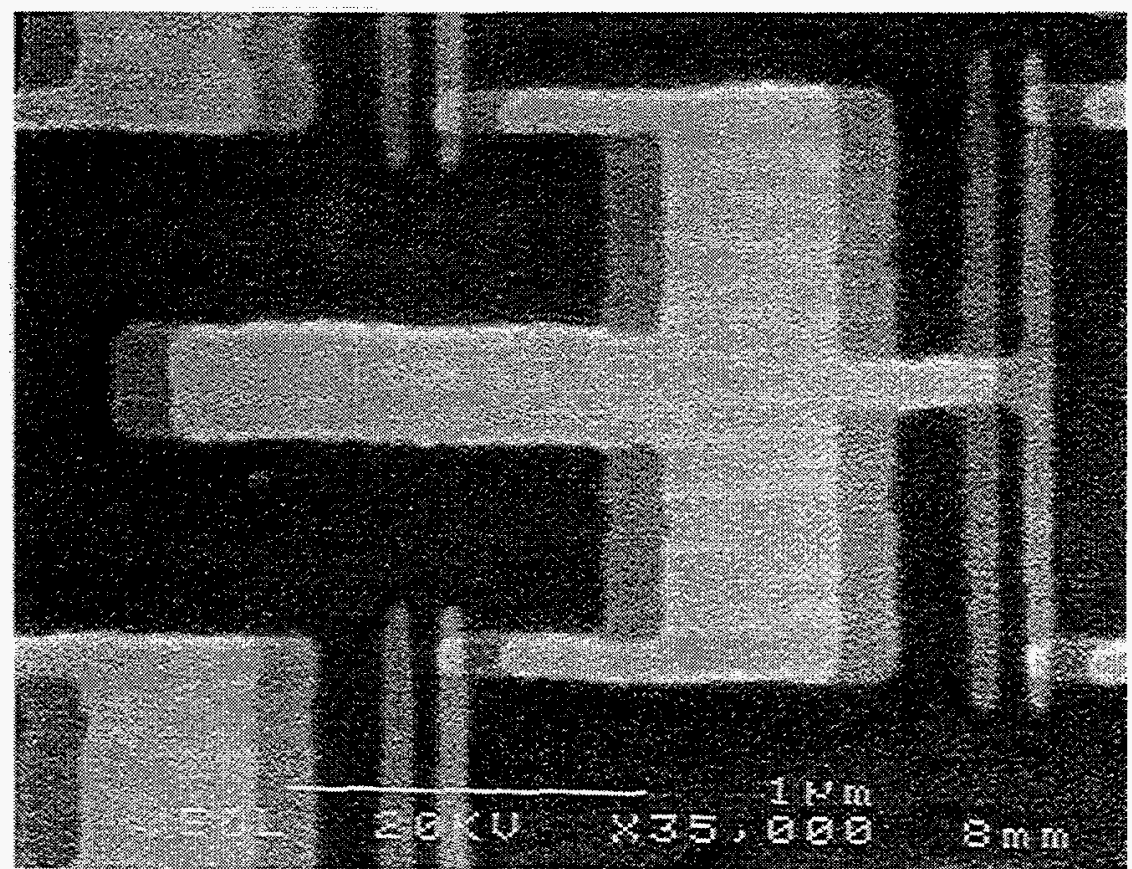

Figure 6.6: SEM micrograph of an island from a $38 \times 40$ island $2 \mathrm{D}$ array. The island is linked to four neighboring islands by junctions that are formed at the intersections of the thin horizontal and vertical lines. 


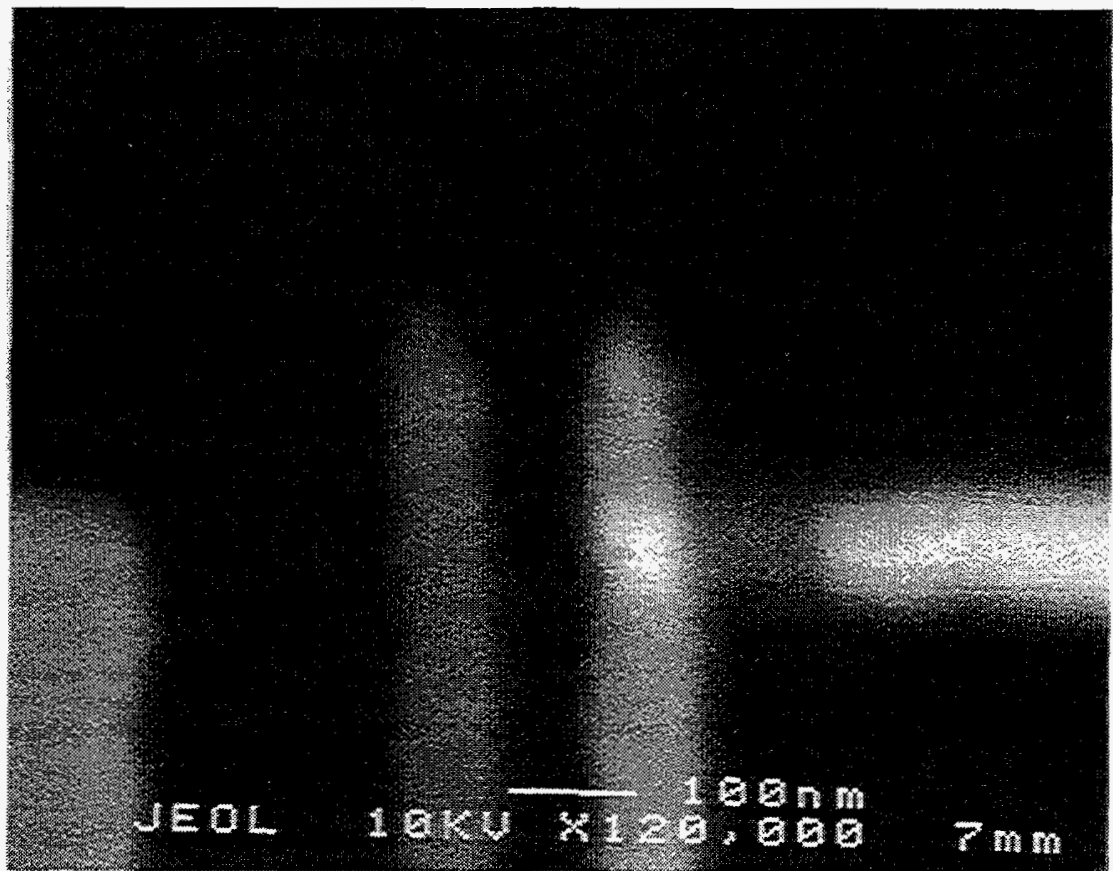

Figure 6.7: SEM micrograph of a single junction. The junction is formed in the bright area where the horizontal and vertical lines overlap. The area of the junction is approximately $60 \times 80 \mathrm{~nm}^{2}$. 

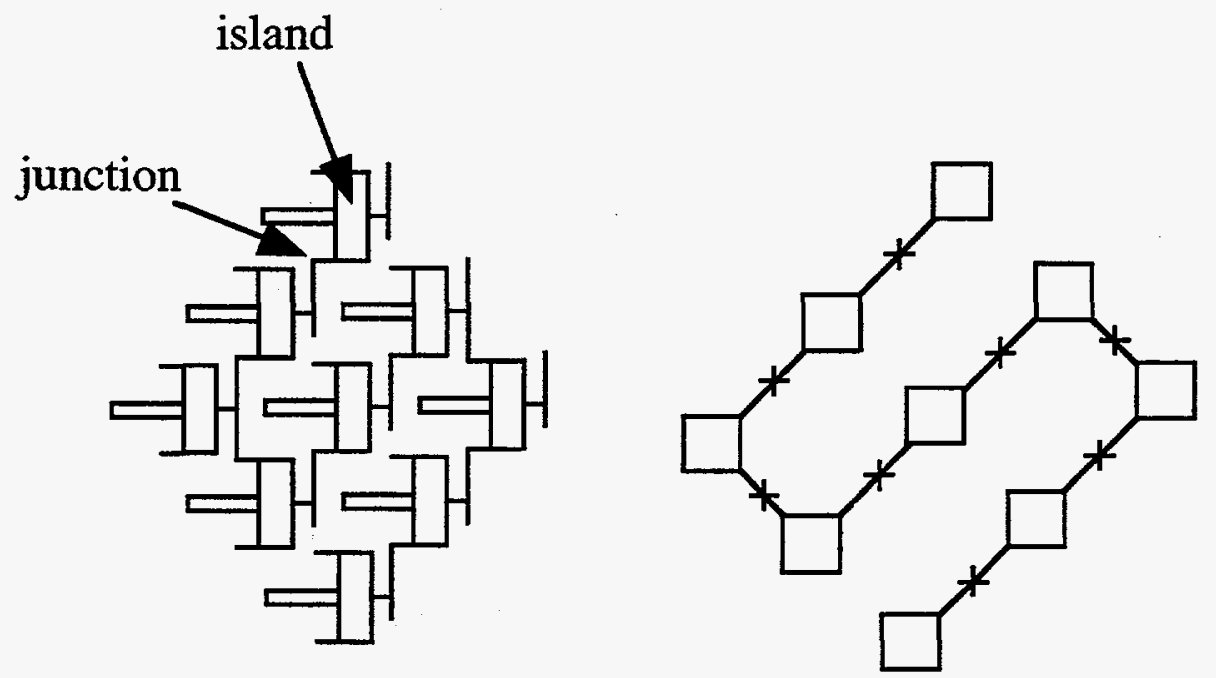

Figure 6.8: Schematic drawings of a small one-dimensional array. The drawing on the left shows the actual island shapes and junction locations. The drawing on the right is a simplification of the drawing on the left, showing the meandering path taken by the array.

The $1 \mathrm{D}$ arrays use the same pattern as the $2 \mathrm{D}$ arrays, but with one feature of the islands modified slightly so that each island is linked to only two nearest neighbors by junctions. The $1 D$ arrays thus do not follow a straight line, but instead follow a meandering path as shown in Fig. 6.8. We believe 1D arrays with this configuration will behave in the same manner as those with a straight-line configuration. The capacitance between an island and its nearest neighbors to which it is not linked by tunnel junctions is negligible compared to the junction capacitance $C$.

In the $2 \mathrm{D}$ array pattern, bus bars extend across the length of opposite sides of the array, and a small lead connects the bus bar to each island it passes. In the $1 \mathrm{D}$ array pattern, leads connect to the ends of the array and run across the length of opposite sides of the array, but do not connect to the islands they pass.

The pattern exposed at the second highest magnification is that for the intermediate leads that connect the bus bars for the $2 \mathrm{D}$ arrays and the leads for the $1 \mathrm{D}$ arrays to the contact pad pattern. This pattern is the same for both the $1 \mathrm{D}$ and $2 \mathrm{D}$ arrays.

The pattern exposed at the lowest magnification is that for the contact pads to the array. These pads are used to make electrical contact to the arrays. There are two contact the same thickness, the area of the islands in arrays on GaAs substrates must be approximately three times smaller than those on $\mathrm{Si}$ substrates for the island capacitances to ground $C_{g}$ to be approximately the same. 


\begin{tabular}{|l|l|l|l|}
\hline Pattern & Magnification & Dose $\left(\mu \mathrm{C} / \mathrm{cm}^{2}\right)$ & Beam Current $(\mathrm{pA})$ \\
\hline \hline array & $300 \times$ & 150 & 10 \\
\hline intermediate leads & $100 \times$ & 200 & 200 \\
\hline contact pads & $25 \times$ & 600 & 6000 \\
\hline
\end{tabular}

Table 6.2: Typical SEM exposure parameters for the sample pattern for accelerating voltage $20 \mathrm{kV}$.

pads connected to each side of the array, allowing for four-point measurements of the array. The contact pad patterns for the $1 \mathrm{D}$ and $2 \mathrm{D}$ arrays are the same. A smaller contact pad pattern is used for the arrays on GaAs substrates, since the GaAs substrates are smaller than the Si substrates.

Shown in Table 6.2 are the typical SEM exposure parameters used for the array, intermediate leads, and contact pad patterns. The islands on the outer edges of the array require higher exposure doses then the islands in the inside of the array due to the proximity effect (see 6.2.2, "Exposure Considerations").

\section{Sample Exposure Procedure}

A detailed list of steps followed in performing an exposure in the SEM is given in Appendix B. Before loading the resist-coated substrates into the SEM, we put a small amount of silver powder suspended in methanol on opposite sides of the substrate, near the edges, to be used for focussing when setting the SEM parameters for an exposure. The silver powder is applied with a sharpened wooden applicator, and then the substrate is blown dry with nitrogen gas or an Aero-Duster to evaporate the methanol and blow away loose pieces of Ag. We then load the substrate into the SEM and adjust the SEM settings. We perform the exposures at an accelerating voltage of $20 \mathrm{kV}$. We first expose the highest magnification pattern, the pattern for the array. This exposure is performed at a magnification of $300 \times$ with a beam current of $10 \mathrm{pA}$. We then expose the next highest magnification pattern, the bus bars (2D arrays) or leads (1D arrays), at $100 \times$ magnification and a beam current of 200 pA. Finally we expose the contact pads pattern at $25 \times$ magnification and a beam current of $6 \mathrm{nA}$. The sample is then removed from the SEM and taken to our group laboratory for development. 


\section{Development}

After the resist-coated substrate is exposed in the SEM, it is developed in $21^{\circ} \mathrm{C}$ 1:3 (methyl isobutyl ketone):(isopropyl alcohol) (MIBK:IPA) for 1 minute. The substrate is then put in a stop bath of IPA for 30 seconds. We then inspect the developed resist under an optical microscope. If the exposure and development appear successful, the substrate is ready for the deposition of metal.

\subsubsection{Shadow Evaporation}

Thin-film deposition is performed in an NRC evaporator for which John Schmidt had constructed an apparatus for tilting the substrate without breaking vacuum. This rig allows thermal evaporation at angles up to $50^{\circ}$ from the normal to the substrate. The evaporator is diffusion-pumped with a liquid nitrogen cold trap. The film thickness is monitored using a quartz oscillator. Although the calibration for this monitor is not known to better than a factor of about 1.5 for most materials evaporated, the thickness measurement is very reproducible [76]. Evaporations can be performed from three different sets of electrodes.

A "Meissner coil" was added to the chamber of the evaporator to provide the option of condensing water vapor and thus lowering the chamber pressure. The Meissner coil is a copper sheet in the shape of a half-circle, with an embedded copper tube running in a meandering path along the surface. The copper tube runs in and out of the chamber through insulated vacuum-tight seals. Liquid nitrogen is run through the tubes of the Meissner coil during the evaporations and oxidation, cooling the coil. Water vapor in the chamber condenses on the coil, reducing the pressure in the chamber. The pressure in the chamber after 90 minutes of pumping without the coil is approximately $3 \times 10^{-6}$ Torr. The pressure in the chamber after 90 minutes of pumping with liquid nitrogen running through the coil for the last 5 minutes is approximately $8 \times 10^{-7}$ Torr.

Also available for use with the evaporator is a homemade "bubbler". The bubbler consists of a large Erlenmeyer flask half-filled with distilled water. The flask is sealed with a glued-in rubber stopper which has two glass tubes connected to plastic tubing running from it. One tube leads to a tank of argon gas, and the other leads to the evaporator chamber. A system of valves allows the user to introduce a controlled amount of $\mathrm{Ar} /$ (water vapor) mixture into the chamber if so desired. 


\section{Evaporation and Oxidation Procedure}

Thin films of $\mathrm{Al}$ are evaporated onto the resist-masked substrates to make the arrays of junctions. First, conical-shaped 3-stranded-wire tungsten baskets (R. D. Mathis Co., Long Beach, CA) are clamped between two sets of electrodes. Three inches of 0.06 inch diameter $99.999 \% \mathrm{Al}$ wire is wound into small balls and placed in each of the $\mathrm{W}$ baskets. The substrate is clamped onto the platform that allows it to be tilted. The vacuum chamber is then closed and diffusion pumped for typically 1 hour. At the end of this hour, the Meissner coil is optionally used to lower the water vapor pressure in the chamber. A layer of approximately $20 \mathrm{~nm}$ of $\mathrm{Al}$ is deposited at an angle of $+11.6^{\circ}$ from the normal of the substrate. A gas mixture of $\mathrm{O}_{2} / \mathrm{Ar}$ (typically $5 \% / 95 \%$ ) is then introduced into the chamber to oxidize the deposited Al. The "bubbler" is optionally used at this point to introduce more water vapor into the chamber. The gas mixture is left in the chamber for a specified time (typically 2 minutes) and is then pumped out. Next, approximately $35 \mathrm{~nm}$ of $\mathrm{Al}$ is deposited at an angle of $-11.6^{\circ}$ from the normal of the substrate. The resulting product of the two evaporations with an intervening oxidation is an array of thin $\mathrm{Al}$ islands linked by thin $\mathrm{Al}_{\mathrm{x}} \mathrm{O}_{\mathrm{y}}$ layers that form the tunnel junctions.

\section{Tunnel Junction Resistance}

The resistance of the small tunnel junctions formed depends linearly on the inverse of the junction area and exponentially on the thickness of the oxide. We can control the area of the tunnel junctions to within approximately $\pm 15 \%$ with electron-beam lithography. The thickness of the oxide formed depends on many factors, including the pressure of $\mathrm{O}_{2}$ introduced into the chamber during the oxidation and the time it remains in the chamber. We can control these two factors very well. Another factor that controls the rate of oxidation and thus the thickness of the oxide is the temperature of the substrate. Since we do not have a heating or cooling system on our tilting sample mount, we can not control this factor. Prior to the time an air conditioner was installed in the room with the evaporator, the temperature was at least somewhat controlled by the outside temperature. Another factor that greatly influences the rate of oxidation is the water vapor pressure in the chamber. We could not control this factor very well. At one point during this experiment, we had difficulty making low enough resistance junctions (we wanted a junction resistance of approximately $200 \mathrm{k} \Omega$ but were obtaining $1 \mathrm{M} \Omega$ ), despite oxidizing with low oxygen pressures and using 
very short oxidation times. We then installed the Meissner coil and were able to obtain the junction resistances we desired. The evaporator was then moved to a different room, and we began having difficulty obtaining high enough junction resistances (at that time we wanted approximately $10 \mathrm{k} \Omega$ junctions but were obtaining $500 \Omega$ ). We then installed the "bubbler" and were able to obtain the junction resistances we desired. We now routinely use the Meissner coil, since it seems to give very reproducible junction resistances.

\section{Liftoff}

Once the $\mathrm{Al}$ evaporations and oxidation are performed, we remove the remaining resist and extra Al by "liftoff". Liftoff involves soaking the substrate in acetone for 1 hour and then applying ultrasound for approximately 10 minutes. Since the resist is soluble in acetone, the resist and metal on top of it come away from the substrate when it is soaked in acetone. Any unwanted $\mathrm{Al}$ still loosely attached to the array is removed by ultrasound with no damage to the array. The array is then blown dry with nitrogen gas and is ready to be mounted and tested. 


\section{Chapter 7}

\section{Dilution Refrigerator}

\subsection{Introduction}

We perform low temperature measurements of the arrays of submicron Josephson junctions over a ground plane in a dilution refrigerator. The refrigerator consists of an Oxford Model 75 dilution unit, a homemade cryostat (including a $1 \mathrm{~K}$ pot), and a homemade ${ }^{3} \mathrm{He} /{ }^{4} \mathrm{He}$ circulation system. ${ }^{1}$ The dilution refrigerator system also includes a thermometer and temperature control system, and a $0.4 \mathrm{~T}$ superconducting magnet. Several changes have been made to the dilution refrigerator system in the past four years, including the design of a new sample mount and installation of a new thermometer. A description of the dilution refrigerator system is given in the next sections.

\subsection{General Configuration}

The sample mount, including a sample holder, $\mathrm{RuO}_{2}$ thermistor, and microwave filters for the sample leads, screws into the tail section of the refrigerator. A copper heat shield separates the sample mount from the vacuum can. A homemade $0.4 \mathrm{~T}$ superconducting magnet is positioned on the vacuum can so that it is approximately centered on the sample holder. Eight sample leads and one high voltage lead run from the sample mount. The sample leads are 2 sets of twisted quads of 0.003 inch diameter manganin wire wound as closely together as possible to reduce interference. In the ${ }^{4} \mathrm{He}$ bath, 4 -conductor cable is used because it was found to generate the least amount of microphonic current noise [76].

\footnotetext{
${ }^{1}$ The ${ }^{3} \mathrm{He} /{ }^{4} \mathrm{He}$ circulation system was designed and made by John Schmidt and Mark Ferrari in $1985-86$.
} 
Removable microwave filters are installed just above the vacuum can top in the ${ }^{4} \mathrm{He}$ bath. These filters are made by filling a Pomona box with Stycast 1266 mixed with an equal weight of $\mathrm{Cu}$ powder. Hermetic connectors are epoxied into the ends of the box. Eight separately-coiled \#29 insulated $\mathrm{Cu}$ wires run through the filter from one connector to the other. Removable low-pass RC filters are installed just above the microwave filters. These filters, potted in Stycast 2850 FT mixed with stainless steel powder to attenuate microwave frequency transmission around the filters, have their $3 \mathrm{~dB}$ point at $16 \mathrm{kHz}$ [76].

Inside the vacuum can, the wiring consists of 0.003 inch diameter manganin wires, and each set of four wires is shielded from the other sets. The wires are heat sunk at the exchanger plate (which is at a temperature of about $120 \mathrm{mK}$ ). More 0.003 inch diameter manganin wire is used to make the final connection to the sample mount at the mixing chamber [76].

To bring high voltage to the sample (for depleting the electrons in the 2DEG substrates via a metallic back gate), a setup previously used to bring microwaves down to the sample was modified. The existing microwave connections are used, since they are rated to higher voltages than we need. Coaxial cable with a teflon dielectric is used to take the high voltage from room temperature to the ${ }^{4} \mathrm{He}$ bath. Manganin wire, 0.003 inch in diameter, inside teflon tubing is used to bring the high voltage down through the ${ }^{4} \mathrm{He}$ bath to the sample holder. This wire is heat sunk at the $1 \mathrm{~K}$ pot, exchanger plate, and mixing chamber.

The dilution refrigerator has a base temperature of about $20 \mathrm{mK}$. The temperature is measured with a calibrated $\mathrm{RuO}_{2}$ thermometer on the sample mount. The refrigerator is suspended by bungee cords to reduce vibration and is housed within a $\mathrm{Cu}$ screened room to eliminate radio frequency interference. A mu-metal shield can be placed around the refrigerator dewar to reduce magnetic field noise. The gas handling system is located outside of the screened room, and care is taken to avoid mechanically coupling vibrations produced by the pumps to the refrigerator. The ${ }^{4} \mathrm{He}$ hold time of the dewar is typically 20 hours.

\subsubsection{Heat Sinks}

The heat sinks for the sample leads were made by John Schmidt and Andrew Cleland by laminating 0.002 inch $\mathrm{BeCu}$ sheet (chosen primarily because it is a resistive 
alloy), a Kim-wipe tissue, and a $1 / 8$ inch $\mathrm{Cu}$ plate (to be clamped to the sinking point), bonded together with Stycast $2850 \mathrm{FT}$ epoxy. The BeCu was patterned into $1 \mathrm{~mm} \times 20$ mm strips using standard printed circuit board lithography techniques. The porous Kimwipe aided in avoiding electrical shorting between the $\mathrm{BeCu}$ strips and the $\mathrm{Cu}$ plate while providing no effective additional material interfaces presenting Kapitza thermal resistance. A simple model by Andrew Cleland showed that this system formed an effective heat sink when used at the heat exchanger plate of the mixing chamber [76].

The heat sinks for the high voltage lead were made by Alex Rimberg. They consist of the following materials, from the bottom up: OFHC Cu plate, undoped $\mathrm{Si}, 0.002$ inch $\mathrm{BeCu}$, undoped $\mathrm{Si}$. The undoped Si was epoxied to the material below it with a thin layer of Stycast 1266. Undoped Si was used because, since it is a single crystal, it has good thermal conductivity while still providing good electrical isolation. Since the thermal conductivity of $\mathrm{Si}$ is much larger than that of the epoxy or that associated with the Kapitza resistance, it can be ignored when considering the thermal conductivity to the $\mathrm{Cu}$ plate.

\subsection{Sample Mount}

The sample mount which screws into the tail of the refrigerator was redesigned by Alex Rimberg to add more filtering for the sample leads and to include a new calibrated $\mathrm{RuO}_{2}$ thermometer. A diagram of the new sample mount is shown in Fig. 7.1. The mount, mostly made of $\mathrm{OFHC} \mathrm{Cu}$, consists of the following parts: sample holder, sample space shield, microwave sample lead filters, and $\mathrm{RuO}_{2}$ thermometer with $\mathrm{Nb}$ shield and cancellation coil.

\subsubsection{Sample Holder}

The sample holder is an upside-down-"L"-shaped piece of OFHC Cu (see Fig. 7.2). Samples are mounted on the flat surface on the top of the holder. A piece of cigarette paper is first glued to the mount with rubber cement to provide adequate electrical insulation between the substrates and the sample holder. The samples with Si substrates are then glued to the paper with rubber cement. The samples with GaAs substrates containing a 2DEG are placed on a metallic back gate (a piece of undoped $\mathrm{Si}$ with Au deposited on its surface). The back gate is first glued to the paper with rubber cement, and then the substrate is glued to the back gate with a very thin layer of rubber cement. Indium contacts 


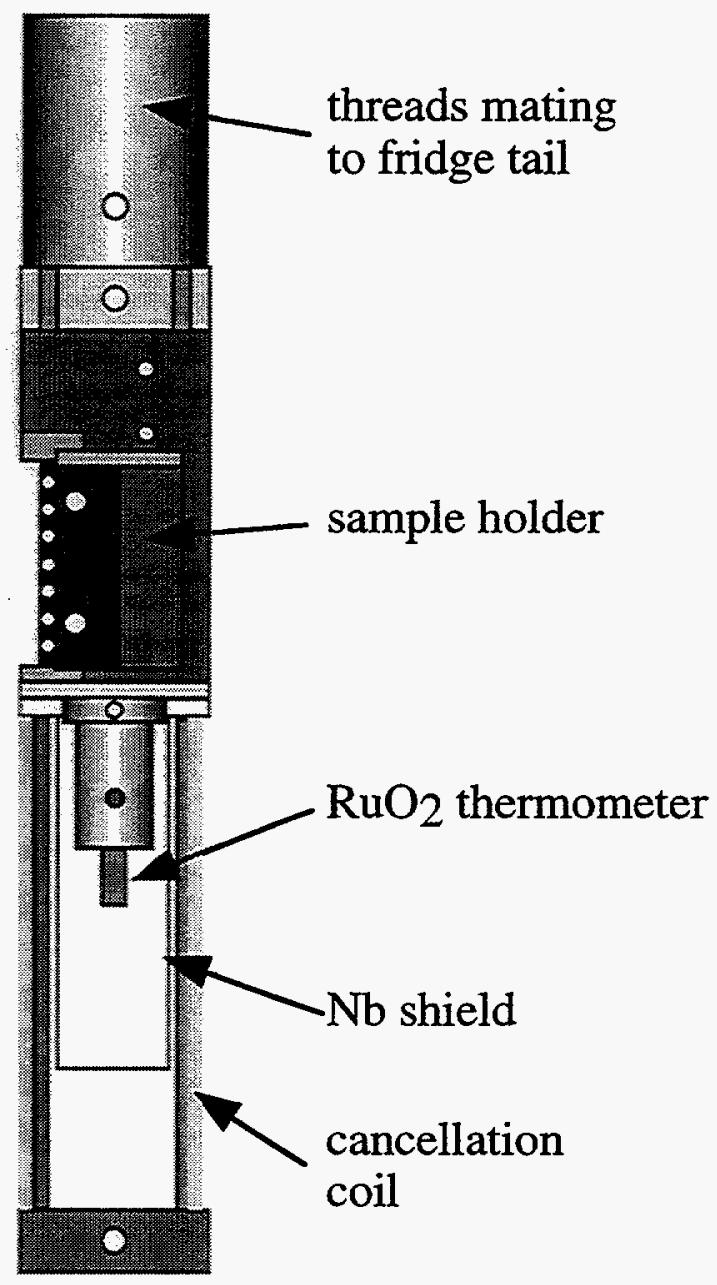

Figure 7.1: Dilution refrigerator sample mount, showing sample holder, $\mathrm{RuO}_{2}$ thermometer, $\mathrm{Nb}$ shield, and cancellation coil. 


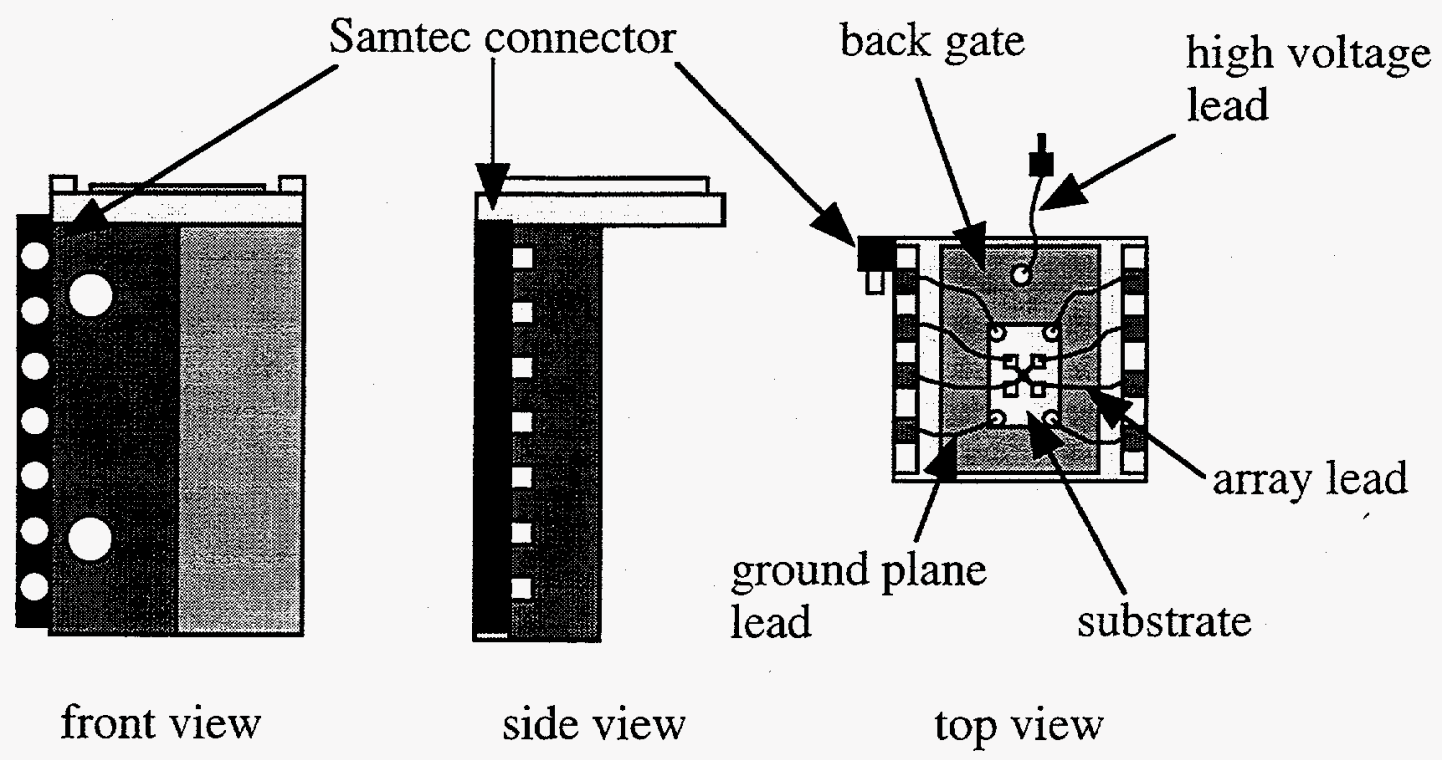

Figure 7.2: Front, side, and top view of a sample holder $(2 \times$ actual size $)$ showing the location of a mounted substrate and back gate.

are then pressed to the lithography-fabricated $\mathrm{Al}$ contact pads leading to the array and to the contacts to the conducting regions (ground planes) of the substrates. Bare $\mathrm{Cu}$ wires that are soldered to brass pads on thin slivers of fiberglass (epoxied with Stycast 2850 FT to the flat surface of the sample holder) are then pressed onto the array and ground plane contacts with more In. Insulated $\mathrm{Cu}$ wires run from the pads on the fiberglass slivers to 8-pin female Samtec connectors epoxied with Stycast 2850 FT to the side of the sample holder. This Samtec connector mates to an 8-pin male Samtec connector epoxied with Stycast 2850 FT to the sample mount. Two holes in the sample holder allow the holder to be screwed onto the sample mount for better thermal contact. Eight 0.003 inch diameter manganin wires leading from the 8 -pin male Samtec connector then run to the two microwave filters. A $\mathrm{Cu}$ shield encloses the sample space containing the sample holder. The high voltage wire enclosed in teflon runs through a small hole in this shield into the sample space and can be directly attached to the back gate of the samples containing a 2DEG.

\subsubsection{Microwave Filters}

Two microwave filters, each filtering four sample leads, are similar to the microwave filters in the ${ }^{4} \mathrm{He}$ bath (see Sec. 7.2). Each of 8 pieces of $40-42$ inch \#29 insulated Cu wire 
is manipulated into a coil shape by coiling it around a $1 / 8$ inch rod. These coiled wires are run through channels in two copper filter housings (four channels in each housing). Each channel is then filled with (50\% Stycast 1266$) /(50 \% \mathrm{Cu}$ powder) (by weight) using a syringe. The microwave filters attach to both the sample side and refrigerator side with Microtech connectors.

\subsubsection{Ruthenium Oxide Thermometer}

A calibrated $\mathrm{RuO}_{2}$ thermistor purchased from Oxford Instruments (Oxford, England) is mounted on a $\mathrm{Cu}$ holder that screws into the bottom of the sample holder. The thermometer is calibrated from $19.8 \mathrm{mK}$ to $4.39 \mathrm{~K}$. We enclose the thermometer in a $\mathrm{Nb}$ shield, since the calibration of the thermometer changes as the applied magnetic field is changed. Since we sometimes wish to operate in a magnetic field of $0.4 \mathrm{~T}$, which is higher than the critical field of $\mathrm{Nb}$, we also add a superconducting cancellation coil around the $\mathrm{Nb}$ shield. The cancellation coil is wound on a specially-machined copper core with 0.0063 inch diameter Cu-clad NbTi (48\% Ti) wire (Supercon, Inc., Shrewsbury, MA). Each layer of wire wound around the core is epoxied into place with Stycast 1266 . The combination of $\mathrm{Nb}$ shield and cancellation coil was tested by replacing the thermistor with a GaAs heterostructure with a 2DEG (sheet density $n_{s} \sim 3 \times 10^{11} \mathrm{~cm}^{-2}$ ) and measuring the Hall resistance of the $2 D E G$ as a function of magnetic field at $4.2 \mathrm{~K}$. To within $\pm 5 \Omega$, the Hall resistance of the $2 \mathrm{DEG}$ did not change when the applied magnetic field was increased from 0 to $0.3 \mathrm{~T}$; therefore the magnetic field inside the shield and cancellation coil did not change by more than approximately $2.5 \times 10^{-3} \mathrm{~T}$ as the applied magnetic field was increased from 0 to 0.3 $\mathrm{T}$. We conclude the shield and cancellation coil satisfactorily shield the $\mathrm{RuO}_{2}$ thermistor.

\subsection{Thermometry and Temperature Control}

The $\mathrm{RuO}_{2}$ thermometer described in the previous section is used to measure the temperature of the sample. Its resistance is measured using a Rochlin bridge [74] which also has a feedback system to provide temperature regulation of the mixing chamber by heating a metal film resistor mounted on the mixing chamber.

Speer carbon resistors are mounted on the still, exchanger plate, and mixing chamber to measure the temperatures at these points. The conductances of these resistors are read by an SHE conductance bridge. These thermometers are used primarily to check the 
status of the refrigerator and as a diagnostic tool when something goes wrong. Thus, the calibration of these thermometers is not crucial.

\subsection{Superconducting Magnet}

A homemade superconducting $0.4 \mathrm{~T}$ magnet is attached to the outside of the vacuum can, positioned so that a sample inside the can will be approximately centered inside the magnet. The magnet was wound by David Cooperberg using Cu-clad NbTi ( $48 \% \mathrm{Ti}$ ) wire. The exact calibration of the magnet is uncertain, but can be inferred from measurements of the magnetic field outside of the magnet and magnetic field dependent measurements of the arrays (see Chap. 4). Leads from the magnet run out to room temperature, where they connect to an HP 6023A DC Power Supply. The magnet has a persistent switch consisting of a carbon resistor around which is wound a section of the magnet wire (stripped of insulation and $\mathrm{Cu}$ cladding) connecting the leads of the magnet. The resistor and wire are epoxied together with Stycast 1266. The heat switch and various superconducting joints had to be remade many times by Çăgliyan Kurdak and Alex Rimberg, due to various problems with the magnet operation. 


\section{Chapter 8}

\section{Measurement Techniques}

\subsection{Introduction}

After the completed arrays over a ground plane are mounted on the sample holder and cooled down to low temperatures in the dilution refrigerator, various electronic measurements of both the arrays and the ground planes are performed. Measurements of the arrays include DC measurements of the current-voltage $(I-V)$ characteristics of the array and $\mathrm{AC}$ measurements (using a lock-in technique) of the zero-bias resistance $R_{0}$ of the arrays. These measurements are all performed using a four-probe technique. Measurements of the ground planes are primarily measurements of resistivity (in both diagonal and Hall configurations) using a lock-in technique. For some measurements of the arrays with the islands in the superconducting state, a small magnetic field (up to $\approx 5 \times 10^{-4} \mathrm{~T}$ ) is applied perpendicularly to the array. For measurements of the arrays with the islands in the normal state, the applied magnetic field is kept at its maximum value of approximately $0.4 \mathrm{~T}$, with the magnet in persistent mode. With the islands in the normal state, the array voltage $V$ versus front gate voltage $V_{F G}$ is also measured to determine the capacitance to ground $C_{g}$ of the islands. The resistance of the ground plane $R_{g}$ of the samples with a $2 \mathrm{DEG}$ is changed by applying a high voltage between the 2DEG and a metallic back gate.

As mentioned in Chapter 7, the sample leads (including the array and ground plane leads) are filtered by microwave and radio-frequency filters at $4.2 \mathrm{~K}$ and by another set of microwave filters at the mixing chamber temperature. The electronics used to measure the arrays and ground plane are all battery powered, except for a Stanford Research SR850 lock-in amplifier and a Macintosh Quadra 650 computer which collects the data. Radio- 


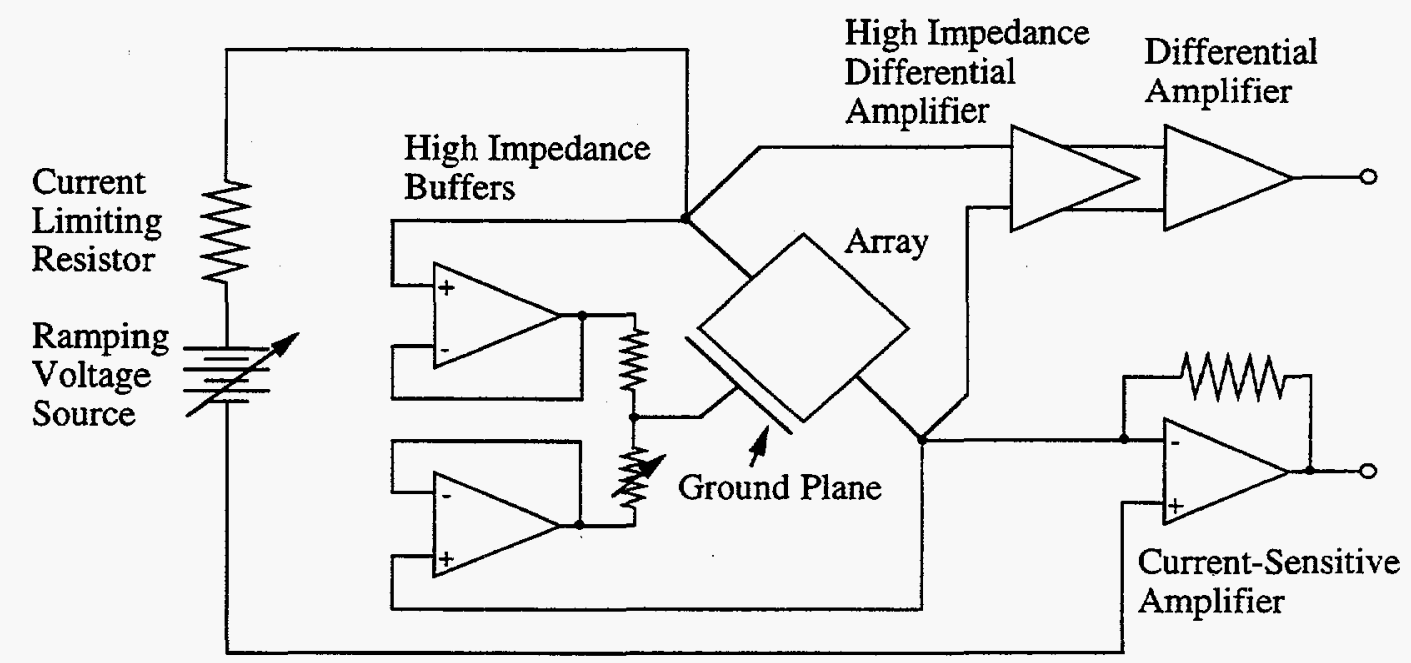

Figure 8.1: Circuit for measuring the DC $I-V$ characteristics of an array.

frequency $\pi$ filters at room temperature reject any digital noise from the computer. All the battery powered electronics are in the screened room with the dilution refrigerator. A mu-metal shield is placed around the sample space when we measure the arrays with the islands in the superconducting state (the mu-metal shield is put in place before cooling the refrigerator to a temperature of $4.2 \mathrm{~K}$ or below). In a typical run, measurements on an array with the islands in the superconducting state are performed first. The mu-metal shield is then removed, the magnet is ramped to full field $(0.4 \mathrm{~T})$, and measurements are performed on the array with the islands in the normal state.

\subsection{Array Measurements}

\subsubsection{I-V Characteristics of Array}

The DC current-voltage $(I-V)$ characteristics of the $1 \mathrm{D}$ and $2 \mathrm{D}$ arrays with the islands of the array in both the superconducting and normal state are measured. The circuit for the $I-V$ measurements is shown schematically in Fig. 8.1. The array is currentbiased using a large current-limiting resistor (typically $10 \mathrm{G} \Omega$ ) and ramping source [83]. The voltage across the array $V$ is measured with a homemade differential amplifier buffered by a homemade high-impedance $\left(10^{15} \Omega\right)$ differential amplifier. The current from the array is measured by a homemade current-sensitive amplifier with a low-current-noise input. 
When measuring the arrays with a large normal state tunneling resistance $R_{N}$ on $\mathrm{Si}$ substrates, we take measures to prevent the appearance of a common-mode voltage between the array and the ground plane during an $I-V$ sweep. ${ }^{1}$ Homemade high-impedance buffer amplifiers $\left(10^{15} \Omega\right)$ are used to drive a balanced pair of roughly $1 \mathrm{M} \Omega$ resistors with the differential voltage across the array. The average of this voltage is applied to the ground plane. When measuring the arrays with small $R_{N}$ on GaAs substrates, the ground plane is typically connected to the negative current lead of the array.

\subsubsection{Zero-Bias Resistance of Array}

The zero-bias resistance of the arrays, defined as the resistance of the arrays at nearly zero current and voltage, is measured using a standard low-frequency lock-in technique. A small AC signal (typically $1 \mathrm{nA}$ ) is applied to the array from the built-in oscillator of a Stanford Research SR850 lock-in amplifier (at typically $11 \mathrm{~Hz}$ ) through a large current-limiting resistor (typically $10 \mathrm{M} \Omega$ ). The voltage across the array is amplified with a Princeton Research PAR 113 differential amplifier or homemade instrumentation amplifier and returned to the lock-in amplifier. For these measurements, the leads to the ground plane are all grounded.

\subsubsection{Island Capacitance to Ground}

When the islands of the arrays are in the normal state, we also measure the array voltage $V$ versus front gate voltage $V_{F G}$ to determine the capacitance to ground $C_{g}$ of the islands (see Chap. 9). These measurements are performed by current-biasing or voltagebiasing the array and sweeping $V_{F G}$ with the ramping source. $V_{F G}$ is measured with a PAR 113 amplifier or homemade instrumentation amplifier. The current or voltage of the array is measured in the same way as described in the previous sections.

\footnotetext{
${ }^{1}$ See Chap. 3 for a discussion of threshold voltages in symmetrically biased and asymmetrically biased arrays.
} 


\subsection{Ground Plane Characterization}

\subsubsection{Ground Plane Resistivity}

To measure the resistivity of the ground plane (primarily in the samples with GaAs substrates with a 2DEG), a standard low-frequency lock-in technique is used. The measurement setup is the same as that used to measure $R_{0}$ of the arrays. A van der Pauw technique [85] is used to determine the resistance per square $R_{g}$ of the ground plane.

To change the resistance of the ground plane in samples with a $2 \mathrm{DEG}$, a large voltage is applied between the back gate and the 2DEG. As described in Chapter 7, the high voltage is brought from room temperature down to the sample by 0.003 inch diameter manganin wire covered with teflon tubing through various heat sinks and connectors. Outside the screened room, a Kepco Operational Power Supply provides the high voltage through a high-voltage BNC to the screened room. Inside the screened room, the high voltage is brought to the refrigerator by high-voltage cabling through $\pi$ filters. The voltage is measured from the Kepco supply output with a high-voltage probe.

\subsubsection{Sheet Density of 2DEG}

For samples made on GaAs substrates with a 2DEG, the sheet density of the 2DEG is determined by performing Shubnikov-de Haas measurements. Details of this measurement are give in the next section.

\subsection{Magnetic Field Sweeps and Ramping}

For the $2 \mathrm{D}$ arrays with the islands in the superconducting state, we perform measurements of the array and ground plane as a function of the applied magnetic field (applied perpendicularly to the array), at low magnetic fields (up to $\approx 5 \times 10^{-4} \mathrm{~T}$ ). Slow ramps of small currents through the magnet leads are performed with an HP 3325A function generator biasing a $53 \Omega$ resistor. The magnetic field is typically measured with a flux gate magnetometer placed outside the refrigerator dewar directly below the magnet.

When we do not want to sweep the magnetic field, but want to perform a measurement at a specific value of low magnetic field, we sweep to the specified field and then operate the magnet in persistent mode. At one point, we were forced to operate with no 
persistent switch. We then biased the magnet leads with a Keithley 238 High Current Source.

To perform measurements on both the $1 D$ and $2 D$ arrays in the normal state, we drive the islands in the array normal using a larger magnetic field of about $0.4 \mathrm{~T}$. Our homemade magnet requires a current of about $16 \mathrm{~A}$ to reach its peak magnetic field of about 0.4 T. This current is provided by an Hewlett Packard HP 6023A DC Power Supply. When ramping the magnetic field, we are careful not to ramp too rapidly; otherwise, the sample and refrigerator temperature increases dramatically. This warming is mostly due to eddy-current heating of the sample holder and lower part of the refrigerator [73]. This heating is not a problem at the low magnetic fields used in the magnetic field sweeps with the islands of the array in the superconducting state.

To determine the sheet density $n_{s}$ of the 2DEG in the GaAs substrates, Shubnikovde Haas measurements are performed. In these measurements, the four-probe resistivity of the ground plane is measured as the magnetic field is swept up to the full field of $0.4 \mathrm{~T}$. The current through the magnet is swept by an HP 6023A DC Power Supply controlled by an HP 3325A Function Generator. The magnetic field is measured by a flux gate magnetometer placed under the dewar directly below the magnet. Measuring the magnetic field in this manner is sufficient, since for this measurement we are primarily interested in the change in magnetic field and not the absolute value of the field.

\subsection{Data Acquisition and Analysis}

Data is collected on a Macintosh Quadra 650 computer using the data acquisition program Labview 3.0 (National Instruments, Austin, TX). A Labview program was written to sample data via GPIB at evenly spaced intervals (usually 1 second). Data analysis is performed using the commercial graphics package Igor (WaveMetrics, Lake Oswego, OR). 


\section{Part III}

Measurement and Discussion of

Arrays 


\section{Chapter 9}

\section{Scaling Behavior in the}

\section{Current-Voltage Characteristic of One- and Two-Dimensional Arrays of Tunnel Junctions}

\subsection{Introduction}

The transport of interacting objects through quenched disorder is an ubiquitous phenomenon. Several well-known examples, including sliding charge-density waves [39], flux-line lattices in type-II superconductors [8], and fluids in disordered media [61] have been the subject of extensive investigation. Such systems typically display a threshold behavior: below some critical force $F_{c}$ the system is static and the velocity of objects in it is zero, while above $F_{c}$ the system enters a dynamic conducting state in which the objects move, producing transport through the system. Fisher suggested that this behavior can be analyzed as a dynamic critical phenomenon [30], and critical exponents associated with the conduction transition have been calculated in a variety of models. Recently Middleton and Wingreen (MW) [63] proposed that one- and two-dimensional (1D and 2D) arrays of small normal metal islands linked by tunnel junctions, in which transport occurs through the stochastic flow of discrete charges, should provide a novel model system for the study of such dynamic critical phenomena. In these arrays the microscopic degrees of freedom 
and their range of interaction are well understood and under good experimental control. Furthermore, the possible sources of microscopic disorder are clear. Thus, such arrays offer a unique opportunity to investigate the relationship between microscopic parameters and universality classes for dynamic critical phenomena.

\subsection{Middleton and Wingreen Model}

In the model of Middleton and Wingreen (see also Sec. 3.4, Chap. 3), the arrays consist of small normal metal islands linked by tunnel junctions of resistance $R_{N}$ and capacitance $C$ located close to a ground plane to which each island has a capacitance $C_{g}$. We assume $R_{N} \gg R_{Q e}$, where $R_{Q e} \equiv h / e^{2}$ is the resistance quantum for single electrons, and $e^{2} /\left[2 \max \left(C, C_{g}\right)\right] \gg k_{B} T$. The proximity of each island to the ground plane allows us to neglect capacitive coupling between non-neighboring islands $[6,7,37]$. An excess charge placed on an island will polarize surrounding islands; the polarization drops away from the charge exponentially with a screening length $\lambda$ which increases with $C / C_{g}$. The excess charge and its associated polarization constitute a soliton. In the absence of disorder, soliton dynamics in long-screening-length $\left(C \gg C_{g}\right)$ arrays have been studied extensively, both theoretically $[6,7,37]$ and experimentally [21, 82, 65, 22]. However, little theoretical work and to our knowledge no experimental work has been reported in the limit of short screening length $\left(C \ll C_{g}\right)$, with or without the effects of disorder. In this limit MW consider 1D arrays of $N$ islands $(50<N<2000)$ and square 2D arrays of $N \times N$ islands $(40<N<400)$; electrical contact is made via leads on opposite sides of the array. MW include disorder in the form of offset charges $q_{i}$ associated with each island, representing the charge induced by charged impurities scattered randomly throughout the array. Large offset charges will be partially neutralized by an integral number of mobile charges, so that $0<q_{i}<e$. Furthermore, MW assume that the disorder is maximal, that is, $q_{i}$ is independently and randomly distributed between 0 and $e$. When a voltage $V$ is applied between the leads, no current flows below a threshold voltage $V_{T}$, while for $V>V_{T}$ the current-voltage $(I-V)$ characteristic obeys a scaling law:

$$
I \propto\left(V / V_{T}-1\right)^{\zeta}
$$

For infinite arrays in the limit of short screening length $\left(C \ll C_{g}\right)$, they argue analytically that the exponent $\zeta=1$ and $5 / 3$ for $1 \mathrm{D}$ and $2 \mathrm{D}$; their computer simulations for arrays of 
finite size give $\zeta=1.0$ and $2.0 \pm 0.2$ for $1 \mathrm{D}$ and $2 \mathrm{D}$. The threshold voltage (averaged over disorder) increases linearly in the array size as $V_{T}=\alpha\left(C / C_{g}\right) N e / C_{g}$ where $\alpha\left(C / C_{g}\right) \rightarrow$ $1 / 2$ and 0.338 for $1 \mathrm{D}$ and $2 \mathrm{D}$ as $C / C_{g} \rightarrow 0 ; \alpha$ decreases rapidly as $C / C_{g}$ increases [63].

\subsection{Description of Samples}

We report measurements of the $I-V$ chararteristics of a single $1 \mathrm{D}$ array and a single $2 \mathrm{D}$ array designed to lie in the short-screening-length limit. The arrays consist of $\mathrm{Al}$ islands linked by $\mathrm{Al} / \mathrm{Al}_{\mathrm{x}} \mathrm{O}_{\mathrm{y}} / \mathrm{Al}$ tunnel junctions and are fabricated with electron-beam lithography and a shadow evaporation technique [24]. The electrical leads are separated by $N=440(1 \mathrm{D})$ and $N=38(2 \mathrm{D})$ islands; the $2 \mathrm{D}$ array is 40 islands wide (the $2 \mathrm{D}$ array has 38 columns and 40 rows of islands). The substrates, which act as the ground plane, are degenerately-doped Si thermally oxidized to a thickness of $102 \pm 5 \mathrm{~nm}$ to provide electrical isolation. Each substrate has four ohmic contacts, allowing electrical contact to the ground plane. The islands were designed to give $C_{g} \approx 1.5 \mathrm{fF}$. Typical measured junction areas were approximately 70 by $80 \mathrm{~nm}^{2}(1 \mathrm{D})$ and 70 by $70 \mathrm{~nm}^{2}(2 \mathrm{D})$. Other work [21,84] implies a specific capacitance of $\approx 70 \mathrm{fF} / \mu \mathrm{m}^{2}$ for junctions with size and resistance similar to ours, leading us to expect $C \approx 0.35 \mathrm{fF}$. Disorder in our arrays arises from variations in the junction resistance and capacitance as well as from offset charges $q_{i}$; measured junction areas vary by roughly $20 \%$. Since we have no control over the naturally occurring $q_{i}$, it is not clear which form of disorder dominates.

\subsection{Description of Experimental Setup and Measurements}

We made electrical measurements in a dilution refrigerator at temperatures of 30 $\mathrm{mK}$ to $40 \mathrm{mK}$, using a four-probe technique. The sample leads were carefully filtered by microwave [60] and radio-frequency filters at $4.2 \mathrm{~K}$, and a second set of microwave filters at the mixing chamber temperature. The measurement electronics were battery powered, except for a plotter used to digitize the data. Radio-frequency $\pi$-filters at room temperature were used to reject any noise from the plotter. We performed all measurements in a screened room, and occasionally recorded data with an analog XY recorder to verify that the digital electronics did not affect the results. The array was current-biased for $V \gg V_{T}$, and approximately voltage-biased for $V \leq V_{T}$ where the array resistance generally exceeded 
the bias resistor, $10^{9} \Omega$. We measured the current with a current-sensitive amplifier with a low-current-noise input, and the voltage across the array with a high-input-impedance $\left(\sim 10^{15} \Omega\right.$ ) amplifier. To bias the array symmetrically with respect to the ground plane, we sampled the voltages $V_{L}$ and $V_{R}$ on the two sides of the array with high-impedance $\left(\sim 10^{15} \Omega\right)$ buffers and applied the average voltage $\left(V_{L}+V_{R}\right) / 2$ to the substrate.

\subsection{Characterization of the Array}

To check the quality of the junctions, we measured the $I-V$ characteristics with the islands of the array in the superconducting state, and found a gap voltage of $370 \mathrm{mV}$ (1D) and $32.0 \mathrm{mV}(2 \mathrm{D})$. This voltage is expected to be $4(N+1) \Delta / e$ where $\Delta$ is the superconducting energy gap. We obtain average values for $2 \Delta$ of $0.42 \mathrm{meV}$ and $0.41 \mathrm{meV}$ for the $1 \mathrm{D}$ and $2 \mathrm{D}$ arrays, indicating the superconducting transition temperature may be slightly above the bulk value. This measurement has especially important implications for the $2 \mathrm{D}$ sample: the average conducting path through the array must pass through 39 junctions, indicating that at most a few were open or shorted. After making all measurements, we inspected the samples in a scanning electron microscope for imperfections such as broken lines; we found none.

To make measurements with the islands of the array in the normal state we applied a $0.4 \mathrm{~T}$ magnetic field perpendicularly to the plane of the arrays. In Fig. 9.1 we show typical $I-V$ characteristics of the $1 \mathrm{D}$ and $2 \mathrm{D}$ arrays. As shown in the insets, the $I-V$ characteristics at high current are linear, yielding an average asymptotic junction resistance $R_{N}=188 \mathrm{k} \Omega(1 \mathrm{D})$ and $138 \mathrm{k} \Omega(2 \mathrm{D})$. Both values of $R_{N}$ are significantly larger than the resistance quantum for single electrons $R_{Q e} \approx 25.8 \mathrm{k} \Omega$ so that effects of cotunneling [5] should be minimal. In previous studies [21, 82, 65, 21] of long-screening-length arrays the offset voltage $V_{\text {off }}$ in the asymptotic regime was used as a measure of the junction capacitance; however in our arrays the value of $V_{\text {off }}$ is dominated by $C_{g}[6,7,37]$ and is not useful as a measure of the junction capacitance.

To measure $C_{g}$ we bias the array just above threshold and measure changes in the voltage across the array as we ramp the substrate voltage. ${ }^{1}$ Changing the front gate voltage $V_{F G}$ changes the effective charge seen by an island. We expect the array voltage to be

\footnotetext{
${ }^{1}$ This is a more direct method of determining $C_{g}$ than using the measured value of $V_{o f f}$ with the results of Ref. [6, 7, 37]; furthermore, our arrays do not lie in an asymptotic limit, so that no analytic expression for $V_{\text {of } f}$ is available.
} 

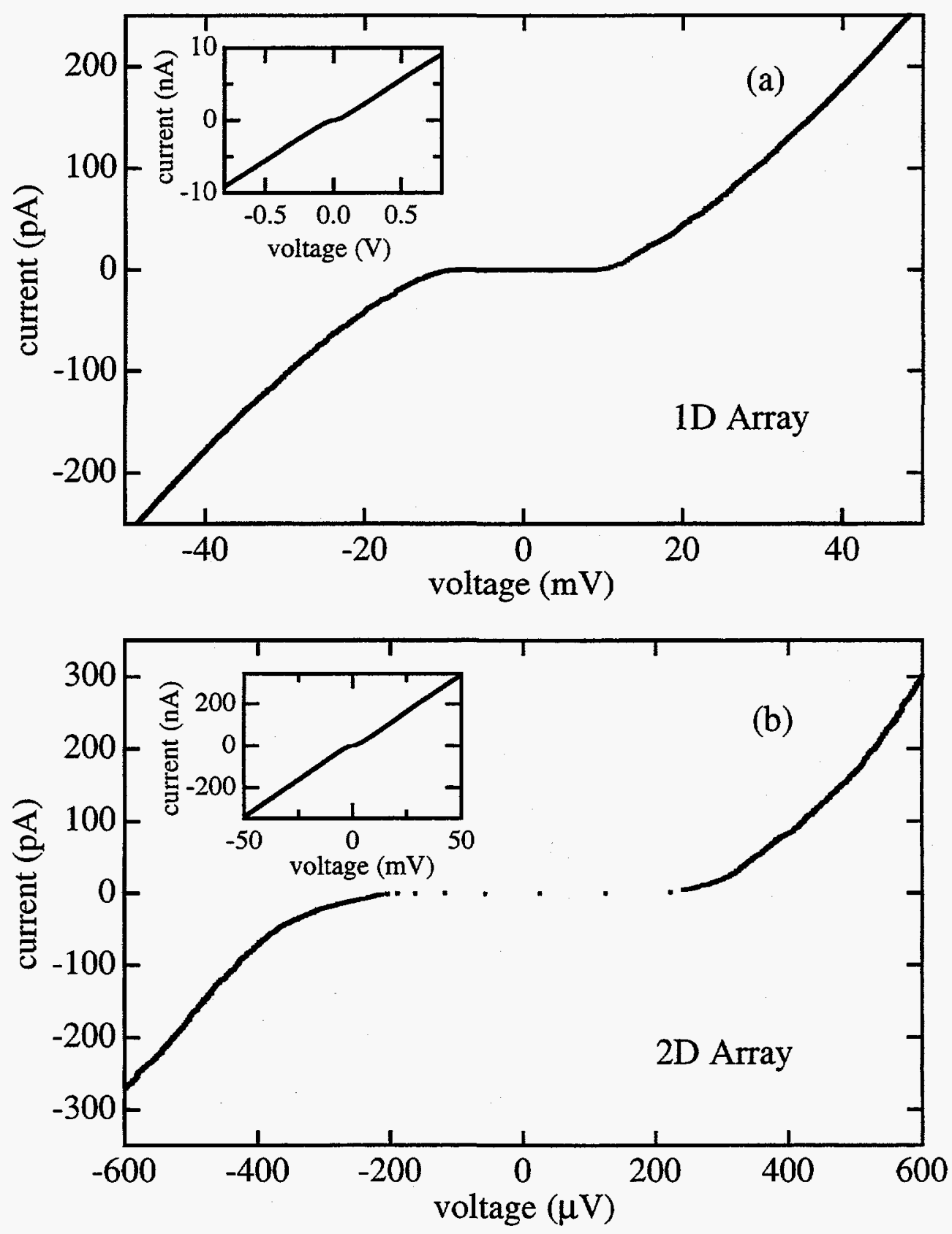

Figure 9.1: $I-V$ characteristics of the (a) $1 \mathrm{D}$ and (b) $2 \mathrm{D}$ arrays with the islands in the normal state. The presence of a threshold voltage above which nonlinear conduction occurs is clearly present in both characteristics. The insets show asymptotic linear behavior at high current. 
periodic in the front gate voltage with period $e / C_{g}$, and for the $2 \mathrm{D}$ array measure a period of $130 \mu \mathrm{V}$, giving $C_{g}=1.2 \mathrm{fF}$ (see Chap. 10 for more discussion of the front gate voltage oscillations in the 2D array). For the ID array the larger array resistance and threshold voltage made it difficult to determine the period precisely. However, the island design and substrate are identical for both arrays so that we expect $C_{g}=1.2 \mathrm{fF}$ for the $1 \mathrm{D}$ array as well. The values of $C$ and $C_{g}$ yield a screening length $\lambda \approx 0.6$ for both $1 \mathrm{D}$ and $2 \mathrm{D}[6,7,37]$.

\subsection{Current-Voltage Characteristics of Array}

Figure 9.1 also shows that the conduction is very small below a threshold voltage $V_{T}$, less than $2.9 \times 10^{-12} \Omega^{-1}$ (1D) and $1.2 \times 10^{-1} \Omega^{-1}$ (2D). Above threshold we see nonlinear conduction in both arrays. We interpret the data in Fig. 9.1 as a dynamic critical phenomenon associated with a transition to a dynamic conducting state at $V_{T}$ when the applied potential is large enough to populate the entire array with electrons, causing current to flow.

\subsubsection{Threshold Voltage}

In measuring $V_{T}$ we encounter two sources of uncertainty. First, for a given set of data there is an uncertainty in $V_{T}$ of roughly $\pm 100 \mu \mathrm{V}$ (1D) and $\pm 10 \mu \mathrm{V}$ (2D) due to rounding of the transition. ${ }^{2}$ Second, the measured value of $V_{T}$ was found to vary on a time scale of hours. This variation could be due to the motion of impurities in the substrate, leading to a different disorder realization and hence a different threshold after sufficient time has elapsed. Alternatively, because the period of oscillations associated with changes in the gate voltage is small (only $130 \mu \mathrm{V}$ ), it is possible that changes in thermal voltages in the sample leads and offset drift in the buffer amplifiers contributed to changes in the measured $V_{T}$. However, in general the changes occurred slowly enough for us to make 4 (1D) or 6 (2D) consecutive sweeps on different current ranges and to piece them together to obtain a single characteristic with no detectable discrepancies. In this way we measured 4 (1D) and 5 (2D) sets of $I-V$ characteristics for the arrays, and found an average $V_{T}=9.4 \pm 0.3 \mathrm{mV}$ (1D) and $230 \pm 20 \mu \mathrm{V}(2 \mathrm{D})$. Calculations in the model of MW using our values of $C$ and $C_{g}$ and

\footnotetext{
${ }^{2}$ Both thermal and quantum fluctuations of charge can round the transition. However, limitations of our measurement technique probably dominate the uncertainty for the $1 \mathrm{D}$ array. Because $V_{T}$ is much larger, its relative uncertainty for the $1 \mathrm{D}$ array is nonetheless much smaller than for the $2 \mathrm{D}$ array.
} 
assuming maximal disorder yield $V_{T} \approx 13 \mathrm{mV}$ (1D) and $580 \mu \mathrm{V}$ (2D) (A. A. Middleton, private communication), larger than the measured values of $V_{T}$ by a factor of about 1.5 and 2.5 , respectively.

Since we do not know the exact amount of disorder present in our arrays, we also compared our results to theoretical predictions in the absence of disorder. In a disorder-free array there is a threshold voltage $V_{s}[6,7,37]$ associated with soliton injection $[6,7,37]$. The voltage $V_{s}$ is an edge effect more or less independent of both array dimension and size for $C \gg C_{g}$. We estimate $V_{s} \approx 110 \mu \mathrm{V}(1 \mathrm{D})$ and $90 \mu \mathrm{V}(2 \mathrm{D})$ for the parameters of our arrays; for the $1 \mathrm{D}$ array, this prediction is two orders of magnitude below our measured value. While in our 2D array $V_{T}$ is only a factor of 2.5 larger than $V_{s}$, this result nonetheless is in marked contrast to results on long-screening-length $2 \mathrm{D}$ arrays for which measured values of $V_{T}$ are typically a factor of 2 to 10 smaller than $V_{s}{ }^{3}$ Finally, in the absence of disorder $V_{s}$ is reduced by a factor of two if the array is asymmetrically biased with respect to ground [21] (see Sec. 3.2.2); we made measurements of $V_{T}$ for the $2 \mathrm{D}$ array with one side fixed at the substrate voltage, but found no significant decrease in $V_{T}$. This result indicates that it is the voltage applied between the ends of the array, rather than the voltage between the edges and ground plane which determines primarily when conduction will occur, implying disorder plays an important role in transport through our arrays.

\subsubsection{Scaling Exponent}

To examine the scaling behavior, we plot current vs. reduced voltage $v=\left(V / V_{T}-\right.$ 1) on a log-log plot, as shown in Fig. 9.2 for two typical sets of data. The threshold voltages were chosen to give a straight line over the widest range of reduced voltage, but in all cases the corresponding current was between one and two times the current noise (in the measurement bandwidth below $0.3 \mathrm{~Hz}$ ) at threshold. As can be seen, the scaling law, Eq. 9.1 is obeyed by both arrays for $0.1 \leq v \leq 8$, corresponding to over 2.5 (1D) and nearly 4 (2D) orders of magnitude in current. We extract the value of the exponent $\zeta$ from the measured slope of the data in the region where Eq. 9.1 is obeyed and find $\zeta=1.36 \pm 0.1$ (ID) and $1.8 \pm 0.16(2 \mathrm{D})$. Above $v \approx 8$ we see a knee in the $I-V$ characteristic and a transition to the linear asymptotic regime.

\footnotetext{
${ }^{3}$ See $[82,21]$. The small measured values of $V_{T}$ are attributed to local lowering of the threshold due to disorder or to quantum fluctuations in charge.
} 


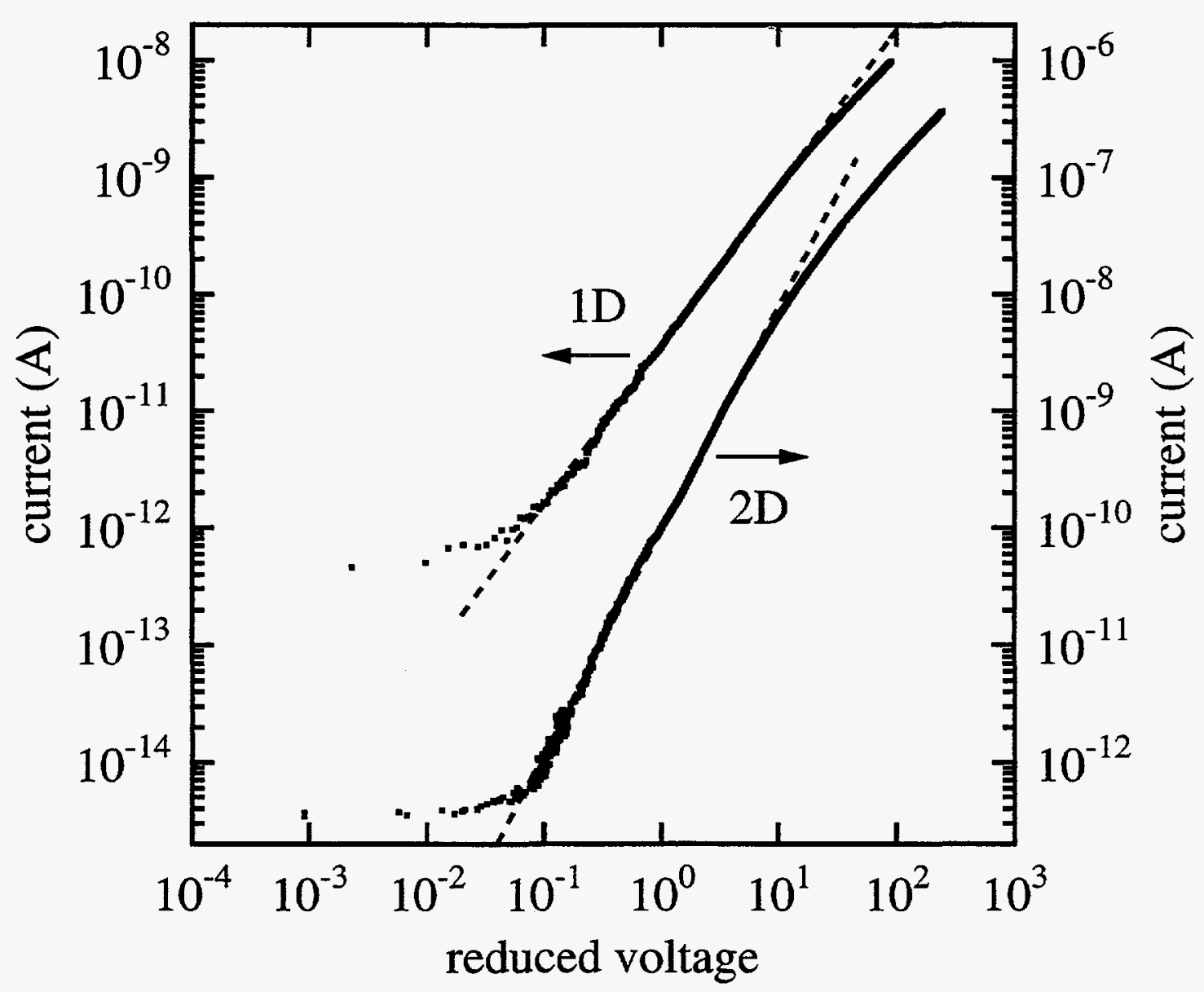

Figure 9.2: Typical composite curves of current vs. reduced voltage $\left(V / V_{T}-1\right)$ plotted on logarithmic axes; $1 \mathrm{D}$ data are plotted on the left axis, 2D data on the right. Dashed lines show the scaling relation, Eq. 9.1, for the values of $V_{T}$ and $\zeta$ fitted to these two data sets. 


\subsection{Discussion}

Our data are qualitatively in good agreement with the simulations of MW. We find threshold voltages significantly larger than those predicted in the absence of disorder, and a larger threshold for the $1 \mathrm{D}$ array, as expected. We also observe scaling behavior above threshold and find a measured value of the exponent $\zeta$ for the $2 \mathrm{D}$ array quite close to the calculated value of $2.0 \pm 0.2$ [63]. While the measured value $\zeta=1.36 \pm 0.1$ for the $1 \mathrm{D}$ array is larger than the calculated value of 1.0 , it is definitely smaller than the measured $\zeta$ for the $2 \mathrm{D}$ array, as predicted.

With regard to the threshold voltages, we note that the agreement between the measured and calculated values of $V_{T}$ is much better for the $1 \mathrm{D}$ array than the $2 \mathrm{D}$ array. In fact, the measured $V_{T}$ in the $2 \mathrm{D}$ array lies closer to that predicted in the absence of disorder. However, disorder in the actual samples may vary more slowly than in the model of MW. If the disorder consists of slowly varying hills and valleys rather than of white noise, we would expect a reduced threshold voltage in the $2 \mathrm{D}$ array since the electrons could flow around significant obstructions. On the other hand, $V_{T}$ for the $1 \mathrm{D}$ array should remain relatively unaffected by slowly varying disorder. It is also possible that variations in the junction capacitance provide the electrons with built-in favorable paths through the $2 \mathrm{D}$ array. Furthermore, despite the fact that $R_{N} \gg R_{Q e}$, cotunneling processes may still play a non-negligible role. In future experiments we shall address these issues by fabricating arrays with different numbers of junctions, intentionally-introduced disorder (see Chap. 10), and larger junction resistance.

We have also neglected the effects of temperature in our data analysis, but we expect that thermal fluctuations will tend to round the transition and may decrease the size of the threshold voltage. We found, however, that a moderate increase in temperature (to roughly $60 \mathrm{mK}$ ) did not cause a significant change in the value of $V_{T}$ or the shape of the $I-V$ characteristic. In fact, quantum fluctuations resulting from the finite size of $R_{N} / R_{Q e}$ may be more important than the thermal fluctuations in this regime [21]. Further measurements are needed to shed light on the temperature dependence of both $V_{T}$ and conduction below $V_{T}$. 


\subsection{Conclusion}

In conclusion, we have presented measurements of the $I-V$ characteristics of $1 \mathrm{D}$ and $2 \mathrm{D}$ arrays of normal metal islands in the short-screening-length limit. Each array exhibited a threshold voltage below which there is almost no conduction and above which the current scales as a power $\zeta$ of the reduced voltage $v$. The values of the threshold voltages $V_{T}=9.4 \mathrm{mV}$ and $230 \mu \mathrm{V}$, and of the exponent $\zeta=1.36$ and 1.80 , in $1 \mathrm{D}$ and $2 \mathrm{D}$ are in qualitative agreement with simulations interpreting the threshold behavior as a dynamic critical phenomenon. Our results are not consistent with a theory based on soliton injection. 


\section{Chapter 10}

\section{Two-Dimensional Array of Tunnel Junctions With Specifically}

\section{Introduced Disorder}

\subsection{Introduction}

Systems of interacting electrons in the presence of disorder have been studied both theoretically and experimentally, but are still not well understood. Examples of such systems include superconductor-to-insulator transitions in thin films $[40,56,68]$ and dynamic critical phenomena in arrays of Josephson junctions [63] and weakly disordered systems such as sliding charge-density waves (for a review, see [39]). One reason such systems are not well understood is the difficulty in naturally occurring systems of varying the degree of disorder without varying other parameters as well. We have developed a method of introducing disorder into a $2 \mathrm{D}$ array of tunnel junctions in a controlled manner, without varying other parameters of the array.

Many types of disorder are present in $2 \mathrm{D}$ arrays of normal metal and superconducting tunnel junctions. One type of disorder is bond disorder, the variation in junction parameters present in every real array due to the imperfect nature of the fabrication procedure. Another related type of disorder is bond dilution, the presence of breaks in bonds in the array. In arrays of superconducting tunnel junctions, there may be positional disorder, where the location of the islands is not regular $[38,34,53]$. In normal metal arrays, there 
may be charge disorder, which takes the form of random offset charges on the islands [63]. In both arrays of superconducting and normal metal junctions, there may be disorder in the form of variations in the sizes of the islands in the arrays. Here we describe our study of a $2 \mathrm{D}$ array of normal metal islands separated by tunnel junctions over a ground plane into which we intentionally introduce disorder in the form of random island areas. These random island areas translate into random capacitances to ground $C_{g}$ of the islands and thus random charging energies $E_{C}$ of the islands.

The array with intentionally-introduced island area disorder is fabricated in the same manner as the arrays with intrinsic disorder used to study dynamic critical phenomena described in Chap. 9. Like the arrays with only intrinsic disorder, the intentionallydisordered array has a large normal state tunneling resistance $R_{N}$. But in contrast to the arrays with only intrinsic disorder, the islands in the intentionally-disordered array are not identical; the islands have random areas. The islands therefore have random capacitances to ground $C_{g}$, since $C_{g}$ is a function of the island area. The charging energy $E_{C}$ of an isiand is a function of the capacitance $C$ of the junctions and $C_{g}$ only, and since $C$ is unchanged, the islands have random charging energies. We therefore introduce disorder in the charging energy of the islands in a controllable and quantifiable way without changing other parameters in the system. Some degree of intrinsic disorder is always present in such arrays, due to variation in junction properties and random offset charges on the islands due to charge impurities in the substrate. We can only add to this intrinsic disorder. But as will be seen in Sec. 10.3, the intentionally-introduced disorder dominates the intrinsic disorder.

\subsection{Description of Array}

The $2 \mathrm{D}$ array of metallic islands separated by tunnel junctions over a ground plane with intentionally-added disorder has approximately the same junction capacitance $C$ and normal state tunneling resistance $R_{N}$ as the $2 \mathrm{D}$ array with only intrinsic disorder described in Chap. 9. The intentionally-disordered array has 38 columns and 40 rows of islands. The junction capacitance is estimated to be $C \approx 0.4 \mathrm{fF}$ from the measured area of junctions in test arrays and the specific capacitance of $\approx 70 \mathrm{fF} / \mu \mathrm{m}^{2}$ determined by other groups [84] for junctions of similar size and resistance. The average normal state tunneling resistance is measured from the asymptotic resistance of the $I-V$ characteristics of the array to be $R_{N} \approx 154 \mathrm{k} \Omega$. The array is made on a degenerately-doped Si substrate, with 


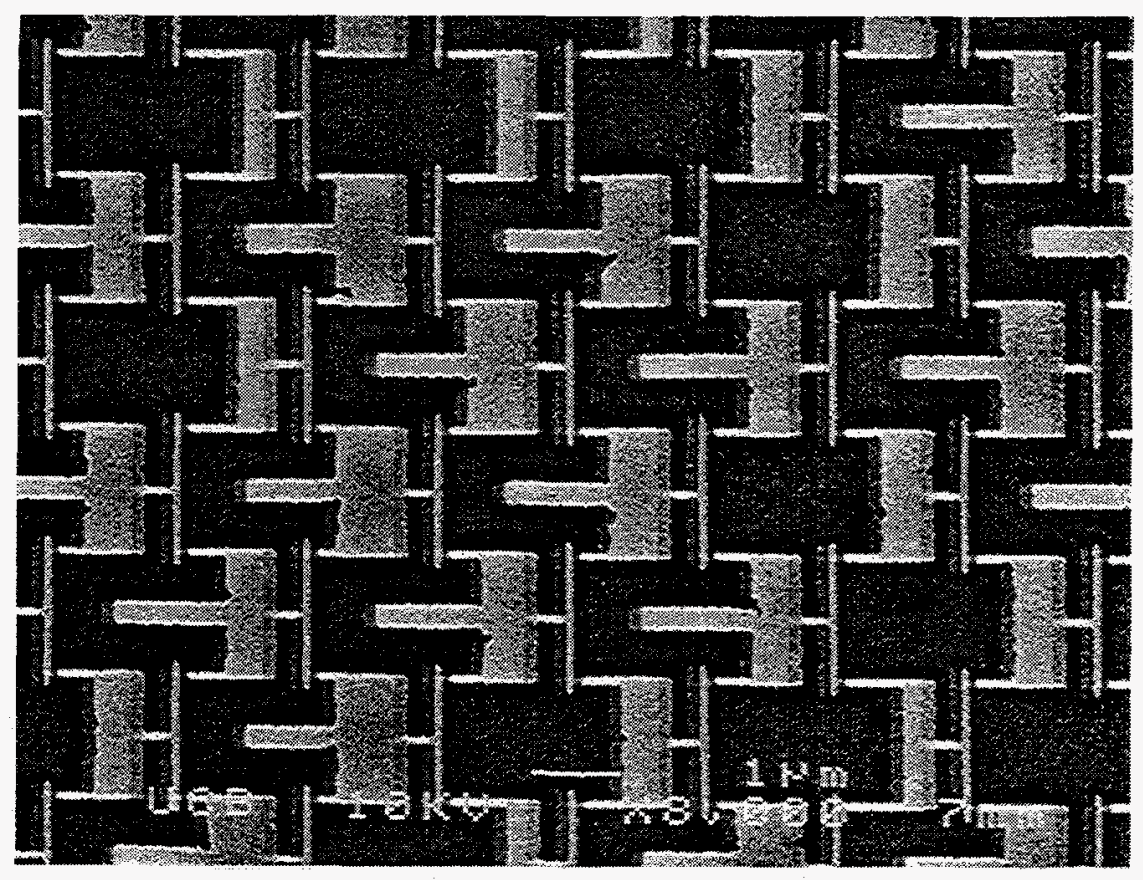

Figure 10.1: SEM micrograph of a portion of a 2D array with intentionally-added island area disorder.

approximately $100 \mathrm{~nm}$ of $\mathrm{SiO}_{2}$ separating the array from the conducting $\mathrm{Si}$ below it. Low temperature measurements of the array were performed in the same manner as for the $2 \mathrm{D}$ array with intrinsic disorder described in Chap. 9.

\subsection{Characterization of Island Area Disorder}

The array with intentionally-added disorder has nine different island areas. The area of a particular island was chosen by a carefully-tested random number generator [69]. The nine island areas are measured from SEM micrographs to be $A=0.8,0.99,1.18,1.37$, $1.58,1.66,1.77,1.85$, and $1.95 \mu \mathrm{m}^{2}$. Shown in Fig. 10.1 is an SEM micrograph of a portion of a $2 \mathrm{D}$ array with the nine different randomly-chosen island areas. The capacitance of an island to ground can be estimated using the formula for the capacitance of a plate with an area $A$ and perimeter $P$ to a ground plane [54] (in SI units):

$$
C \approx \frac{\varepsilon \varepsilon_{0} A}{d}\left[1+\frac{P d}{A} \ln \frac{\sqrt{A}}{2 d}\right]
$$


where $\varepsilon$ is the dielectric constant of the material separating the plates, $\varepsilon_{0}$ is the permittivity of free space, and $d$ is the separation of the plates. For the islands in our arrays, we use $\varepsilon=3.9$ (the dielectric constant of $\mathrm{SiO}_{2}$ ) and $d=0.1 \mu \mathrm{m}$. Using the measured island areas, we calculate the estimated capacitances $C_{g, \text { calc }}=0.49,0.59,0.68,0.83,1.01,1.04,1.10$, 1.13 , and $1.17 \mathrm{fF}$, respectively.

We measure the island capacitances to ground $C_{g}$ of the array in the manner described in Chap. 9. We measure the voltage across the array $V$ versus the front gate voltage $V_{F G}$ (voltage between the array and ground plane) above and near the threshold voltage $V_{T}$. Near $V_{T}, V$ should be periodic in $V_{F G}$ with period $e / C_{g}$. Shown in Fig. 10.2 is typical data of array voltage $V$ versus front gate voltage $V_{F G}$ for the array with only intrinsic disorder and the array with intentionally-added island area disorder. Unlike the data for the array with only intrinsic disorder, no obvious period can be seen in the data for the array with added island area disorder. To determine accurately what periods are contained within the data, we take the power spectral density of $V$ versus $V_{F G}$, as shown in Fig. 10.3. The locations of the peaks in the spectra indicate the island capacitances to ground $C_{g}$ present in the arrays. The power spectrum for the array with island area disorder does not have one single strong peak like the spectrum for the array with only intrinsic disorder. The spectrum for the array with area disorder shows many smaller broadened peaks, as expected, since the array has nine different island areas. The arrows in Fig. 10.3 indicate the calculated values of the capacitance given in the previous paragraph. Although the calculated values do not match the measured peaks exactly, the range of calculated values does agree reasonably well with the range of measured values. We also compare the calculated value of $C_{g}$ for the array with intrinsic disorder with the measured value and get very good agreement. The range of capacitances to ground $C_{g}$ in the array gives an indication of the amount of island area disorder in the array. From these front gate voltage oscillation measurements, we conclude that we have successfully fabricated an array with intentionally-added island area disorder that dominates the intrinsic disorder.

\subsection{Current-Voltage Characteristics of Array}

Shown in Fig. 10.4 is the current-voltage characteristic for the array with added island area disorder. For comparison, the $I-V$ characteristic for the array with only intrinsic disorder is also shown. The blockade regions of both arrays are approximately the 

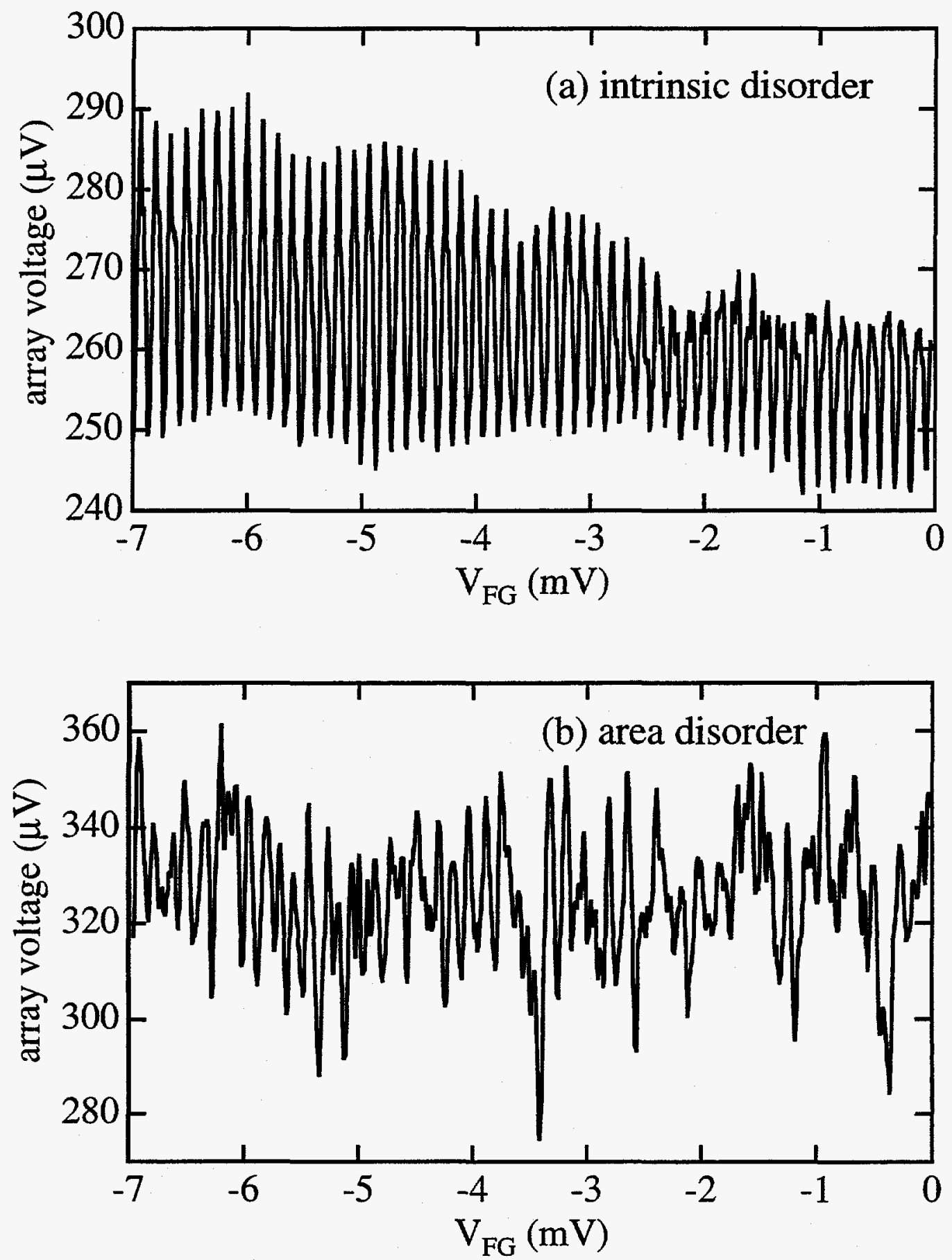

Figure 10.2: Array voltage $V$ versus front gate voltage $V_{F G}$ for arrays with (a) intrinsic disorder and (b) area disorder. 

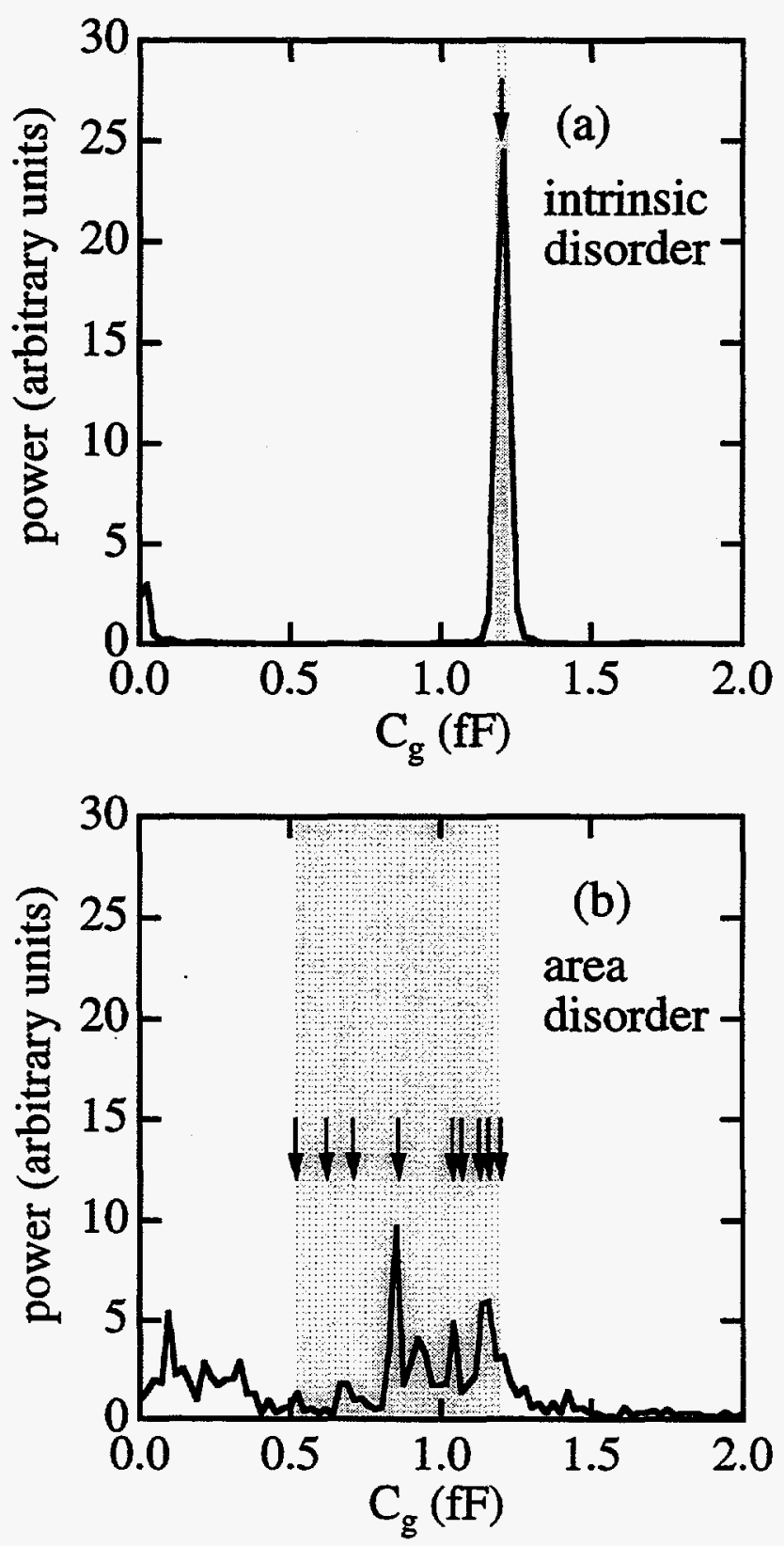

Figure 10.3: Power spectral density of array voltage $V$ versus front gate voltage $V_{F G}$ in units of island capacitance $C_{g}=e / \Delta V_{F G}$ for arrays with (a) intrinsic disorder and (b) area disorder. The locations of the peaks give the island capacitances to ground $C_{g}$ present in the arrays. The arrows indicate the calculated values of $C_{g}$ (see text). The shaded areas give an indication of the amount of island area disorder in the arrays. 

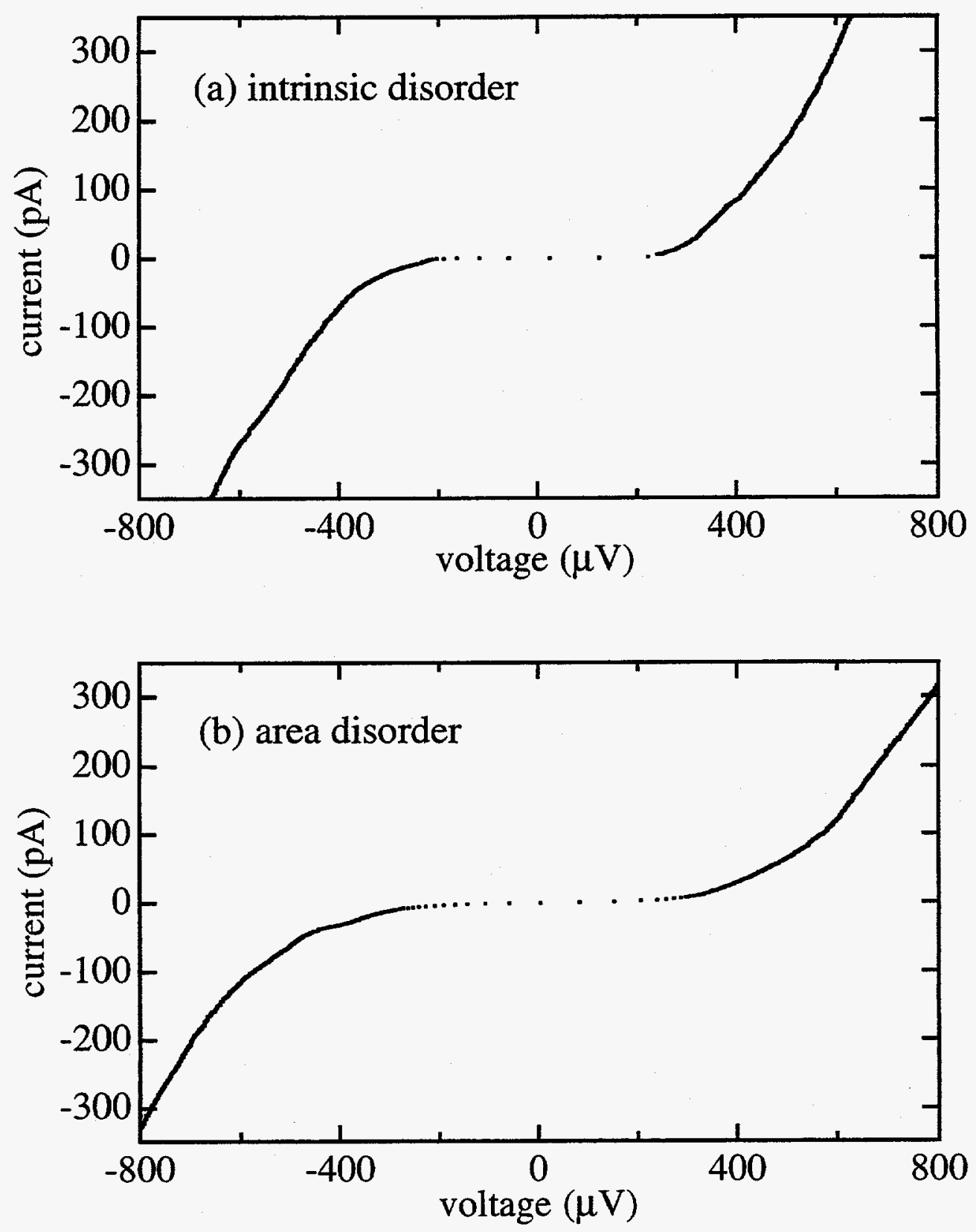

Figure 10.4: $I-V$ characteristics of arrays with (a) intrinsic disorder and (b) area disorder at $T=20 \mathrm{mK}$. 
same size, but the threshold for conduction in the array with area disorder is more rounded and less well-defined.

We also investigate the scaling behavior of the $I-V$ characteristics of the array. We analyze the scaling behavior in the same way as for the array with only intrinsic disorder as described in Chap. 9. Shown in Fig. 10.5 is array current $I$ versus reduced voltage $v=V / V_{T}-1$, with $V_{T}$ being the threshold voltage for conduction, on a log-log scale for both the array with intrinsic disorder and the array with area disorder. The array with added disorder displays scaling behavior over approximately 2 decades, while the array with intrinsic disorder displays scaling behavior over approximately 4 decades. The fitted exponent and threshold voltage for the array with area disorder are $\zeta=2.6 \pm 0.4$ and $V_{T}=250 \pm 40 \mu \mathrm{V}$. Comparing these values to the fitted values of the array with intrinsic disorder, $\zeta=1.8 \pm 0.16$ and $V_{T}=230 \pm 20 \mu \mathrm{V}$ (see Chap. 9), we see the threshold voltages are approximately the same for the two arrays, but the exponent $\zeta$ is about $40 \%$ larger in the array with area disorder.

\subsection{Conclusion}

In conclusion, we are able to successfully fabricate arrays with intentionally-added disorder in the form of random island areas, and thus random island capacitances to ground $C_{g}$ and charging energies $E_{C}$. The island area disorder is controllable, quantifiable, and dominates the intrinsic disorder. The $I-V$ characteristics of the array with added area disorder are different from those for the array with only intrinsic disorder. The sizes of the blockade regions of the two arrays are approximately the same, but the threshold for conduction for the array with area disorder is less well-defined. The scaling law is obeyed over a narrower range for the array with area disorder than for the array with intrinsic disorder. More theoretical work needs to be performed to explain the differences in the $I-V$ characteristics of the two arrays. We hope to continue our study of arrays with intentionally added disorder in the future, as such studies will contribute to the study of transitions as a function of disorder strength. 

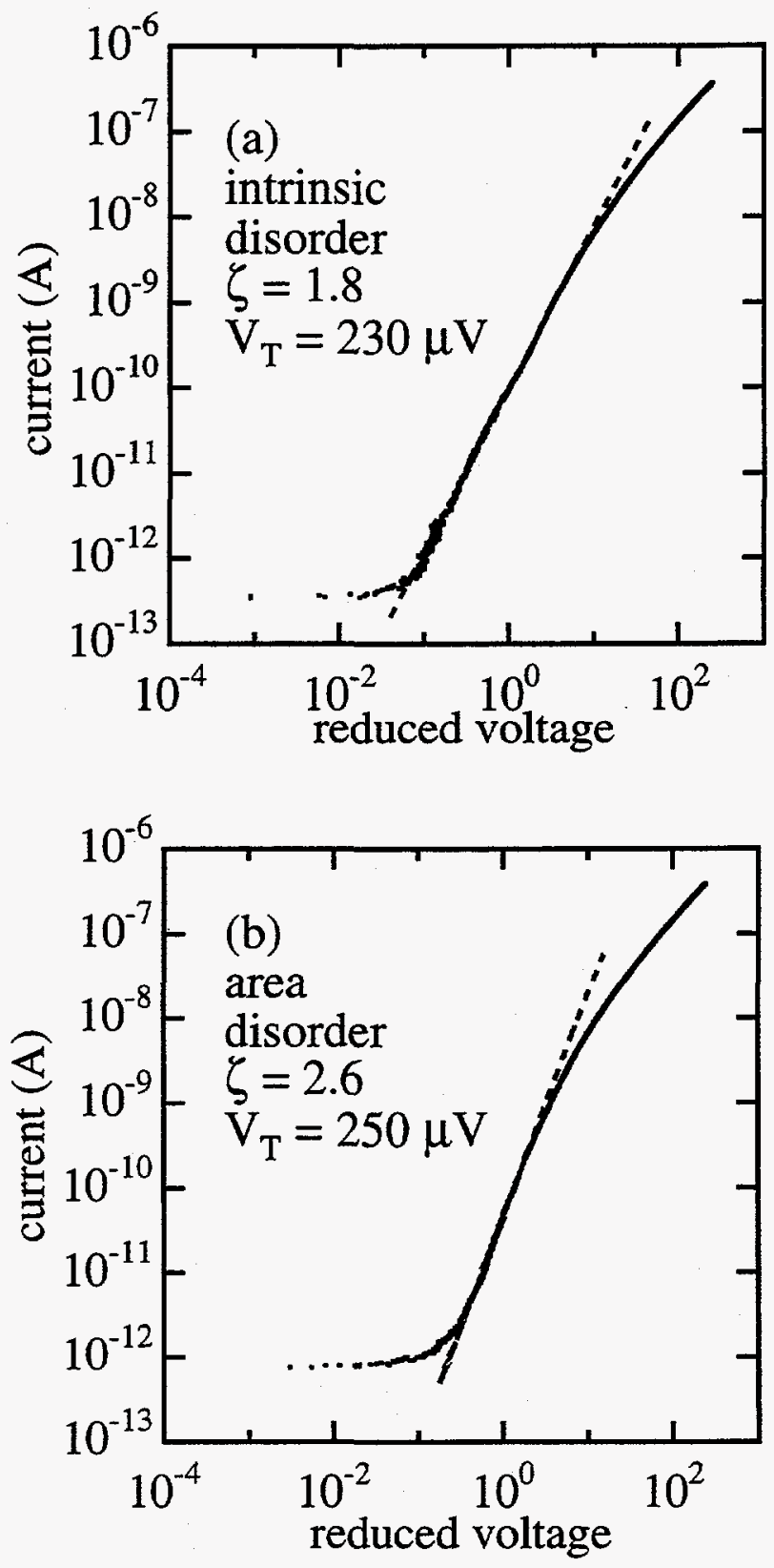

Figure 10.5: Scaling behavior of the $I-V$ characteristics of the 2D arrays with (a) intrinsic disorder and (b) area disorder. Dashed lines show the scaling relation, Eq. 9.1, for the values of $V_{T}$ and $\zeta$ fitted to the data. 


\section{Chapter 11}

\section{Dissipation-Driven}

\section{Superconductor-Insulator}

\section{Transition in a Two-Dimensional Josephson Junction Array}

\subsection{Introduction}

A variety of diverse physical systems, including granular $[67,66,46]$ or homogeneous $[40,56]$ thin films, two-dimensional (2D) Josephson junction arrays [36], and high temperature superconductors [80] undergo a superconductor-insulator (S-I) phase transition as a characteristic resistance of the system in its normal state increases through a critical value on the order of the resistance quantum for Cooper pairs $R_{Q} \equiv h / 4 e^{2} \approx 6.45 \mathrm{k} \Omega$. The transition is quantum mechanical in nature: the increasing normal state resistance is associated with an increase in quantum fluctuations of the superconducting phase. Eventually, these fluctuations destroy global phase coherence and lead to an insulating state. It has been suggested $[16,15,32,26]$ that the S-I transition in these systems could be driven by changes in dissipation; however, there appears to be no unambiguous supporting evidence. In thin films disorder plays a strong role, and recent theoretical work treating these systems as charge- $2 e$ bosons moving in a random $2 \mathrm{D}$ potential $[31,33,94]$ has been met with substantial experimental verification $[40,56,68]$. Furthermore, the physical origin 
of the dissipation is unclear. In the case of Josephson junction arrays, although quasiparticle tunneling [16] at energies large compared to the superconducting gap produces dissipation characterized by the normal state resistance $R_{N}$, it is unlikely that the relevant energy scales are so large $[36,28]$. At lower energies, quasiparticle dissipation is negligible since the subgap resistance is much larger than $R_{N}$. In high temperature superconductors, it has been proposed [26] that an interpenetrating fluid of normal electrons produces the dissipation. However, radiation damage inflicted to increase the normal state resistance probably also increases the disorder and reduces the density of superconducting electrons. Here we describe the observation of a dissipation-driven transition in the current-voltage characteristics of a $2 \mathrm{D}$ Josephson junction array from superconductor-like to insulator-like.

\subsection{Description of Sample}

The sample was a specially designed and fabricated 2D Josephson junction array with 40 rows and 40 columns of islands for which we can continuously vary the dissipation associated with the local electrodynamic environment independently of any other relevant parameters. To provide the variable dissipation, we fabricated the array on a $\mathrm{GaAs} / \mathrm{Al}_{0.3} \mathrm{Gaa}_{0.7}$ As heterostructure in which a $2 \mathrm{D}$ electron gas (2DEG) is located approximately $100 \mathrm{~nm}$ from the surface as shown in Fig. 11.1. The heterostructure was grown on a GaAs substrate using molecular beam epitaxy and consists of the following layers: $500 \mathrm{~nm}$ of $\mathrm{GaAs}, 92 \mathrm{~nm}$ of $\mathrm{Al}_{0.3} \mathrm{Ga}_{0.7} \mathrm{As}$ and $8 \mathrm{~nm}$ of $\mathrm{GaAs}$. The $\mathrm{Al}_{0.3} \mathrm{Ga}_{0.7} \mathrm{As}$ is selectively doped with $\mathrm{Si}$ donors situated $32 \mathrm{~nm}$ from the lower $\mathrm{GaAs} / \mathrm{Al}_{0.3} \mathrm{Ga}_{0.7} \mathrm{As}$ interface, at which the 2DEG forms. The substrate is placed on a metallic back gate. We bias the back gate negatively relative to the $2 \mathrm{DEG}$ with a large voltage $V_{B G}$ to change the sheet density $n_{s}$ of electrons in the $2 \mathrm{DEG}$, and thereby change its resistance per square $R_{g}$. To reduce the required voltage, we thinned the substrate to approximately $230 \mu \mathrm{m}$. The array consists of $\mathrm{Al}$ islands linked in a square lattice by $\mathrm{Al} / \mathrm{Al}_{\mathrm{x}} \mathrm{O}_{\mathrm{y}} / \mathrm{Al}$ tunnel junctions, fabricated using electron-beam lithography and shadow evaporation [24]. The array is characterized by the normal state tunneling resistance $R_{N}$ and capacitance $C$ of the junctions, and the capacitance $C_{g}$ of each island to the 2DEG. The island areas are sufficiently large that $C_{g} \gg C$. We estimate the Josephson coupling energy $E_{J}$ at zero temperature through the relation [3] $E_{J}=\pi \hbar \Delta / 4 e^{2} R_{N}$, where $\Delta$ is the superconducting gap. The charging energy $E_{C}$ of an isolated junction is $E_{C}=e^{2} / 2 C$. The capacitance to ground and junction capacitance 


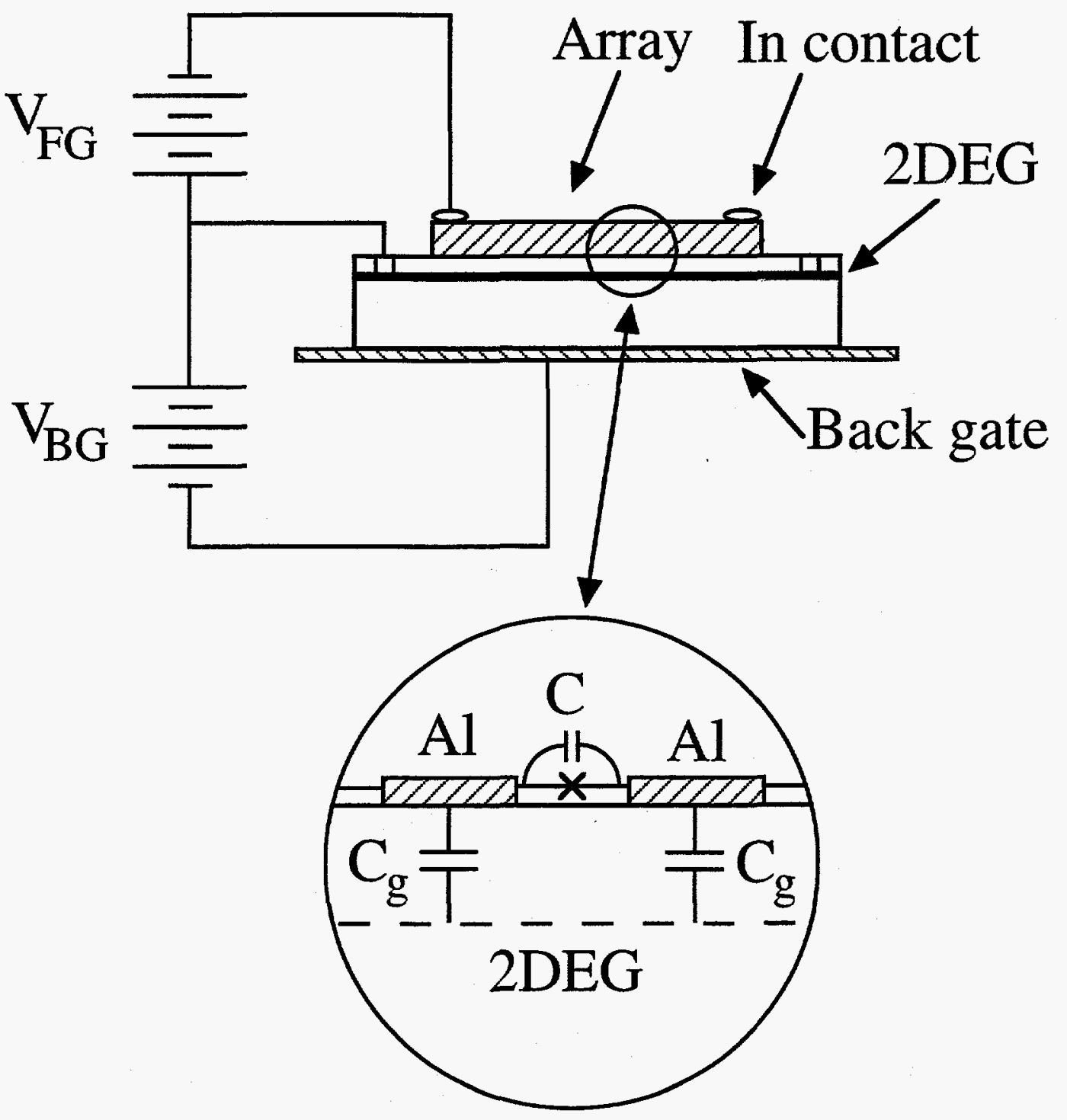

Figure 11.1: Schematic diagram of an array with variable dissipation. The array is fabricated on a GaAs $/ \mathrm{Al}_{0.3} \mathrm{Ga}_{0.7}$ As substrate in which a $2 \mathrm{DEG}$ is located approximately $100 \mathrm{~nm}$ below the surface. Pressed and alloyed In contacts are made to the array and 2DEG, respectively. A voltage $V_{B G}$ between the back gate and $2 D E G$ increases its resistance per square. A voltage $V_{F G}$ between the array in the normal state and $2 \mathrm{DEG}$ allows independent monitoring of the capacitance to ground $C_{g}$. 
determine the energy $E_{C_{\Sigma}}=e^{2} / 2 C_{\Sigma}$ to transfer electrons between neighboring islands; here $C_{\Sigma} \approx\left(C_{g}+5 C\right) / 4 .^{1}$ In the absence of Josephson coupling, we expect a Coulomb gap to appear on the $I-V$ characteristics at a voltage $\pm e N / 2 C_{\Sigma}$, where $N$ is the number of islands in series.

\subsection{Superconductor-Insulator Transitions in Arrays of Jo- sephson Junctions}

Previously, Geerligs et al. studied similar arrays without a ground plane, and spanned the S-I transition with a series of arrays with different $R_{N}$ [36]. In that work, the S-I transition was driven primarily by competition between $E_{C}$ and $E_{J}$. Because the charge $Q$ on a junction and the phase difference $\phi$ across it are conjugate variables they satisfy an uncertainty relation $\Delta \phi \Delta Q \geq 2 e$. When the ratio $E_{C} / E_{J}$ is large, charge is the good quantum variable and phase fluctuations are large, preventing superconductive coupling [1]. Numerous theoretical investigations of arrays, including those based on the quantum XY model (See, e.g., [25]) as well as those which include the effects of capacitance renormalization due to virtual quasiparticle tunneling [28], have predicted the destruction of global phase coherence for $E_{C} / E_{J} \sim 1$. The results of Geerligs et al. suggest a critical value of $E_{C} / E_{J}$ between 1.5 and 2.5.

In the presence of a ground plane of normal electrons we must compare the Josephson energy $E_{J}$ with the island charging energy $E_{C_{\Sigma}}$, which is significantly smaller than $E_{C}$ when $C_{g} \gg C$. We must also consider the dissipation associated with the resistance per square $R_{g}$ of the ground plane. In the heat-bath formalism of Caldeira and Leggett $[12,13]$, dissipation introduces damping of phase fluctuations that is inversely proportional to the resistance of the electrodynamic environment [15,32]. We therefore expect that when $R_{g}$ is small, phase fluctuations will be heavily damped and a large value of $C_{g}$ should promote global superconductivity. When $R_{g}$ is large, the 2DEG will not effectively damp phase fluctuations and in the proper circumstances insulating behavior may result, even if $C_{g}$ is large. In our experiment, we can vary the dissipation continuously in situ without varying

\footnotetext{
${ }^{1}$ To obtain this estimate we calculate the capacitance $C_{n n}$ between adjacent islands, ignoring contributions to $C_{\mathrm{nn}}$ from non-nearest neighbors. We obtain $C_{\mathrm{nn}} \approx \frac{1}{2}\left(C_{g}+5 C\right)$ which is correct to order $C / C_{g}$ for $C_{g} \gg C$. In the opposite limit $C \gg C_{g}$ it can be shown that $C_{\mathrm{nn}}=2 C$ exactly for an infinite array, so that we can write $E_{C}=e^{2} / C_{\mathrm{nn}}$. To be consistent with the notation of Refs. [36, 28], we define $E_{C_{\Sigma}}=e^{2} / C_{n n}$ for the limit $C_{g} \gg C$, giving the expression for $C_{\Sigma}$ in the text.
} 
any other parameters, and span the S-I transition with a single sample at zero magnetic field and fixed temperature.

\subsection{Experimental Setup and Sample Characterization}

We made electrical measurements of the array in a dilution refrigerator at temperatures between 25 and $800 \mathrm{mK}$, using a four-probe technique. The sample leads were carefully filtered by microwave [60] and radio-frequency filters at $4.2 \mathrm{~K}$, and by a second set of microwave filters at the mixing chamber temperature. The measurement electronics were battery powered, except for a microcomputer which collected the data. Radio-frequency $\pi$ filters at room temperature rejected any digital noise. We performed all measurements in a screened room, with a mu-metal shield around the sample space. We measured $R_{g}$ using a van der Pauw technique, and obtained the sheet density $n_{s}$ from Shubnikov-de Haas oscillations at magnetic fields of 0.1 to $0.4 \mathrm{~T}$. At $V_{B G}=0 \mathrm{~V}, n_{s}$ was $2.05 \times 10^{11} \mathrm{~cm}^{-2}$, the mobility was $1.8 \times 10^{5} \mathrm{~cm}^{2} / \mathrm{Vs}$ and $R_{g}=170 \Omega / \mathrm{sq}$. At the maximum applied gate voltage of $V_{B G}=540 \mathrm{~V}, n_{s}$ decreased to $0.7 \times 10^{11} \mathrm{~cm}^{-2}$ while $R_{g}$ increased to $2570 \Omega / \mathrm{sq}$. We expect that the 2DEG is continuous and uniform for $n_{s}$ in this range.

We carefully chose the parameters of the array so that it would be insulating in the absence of a ground plane. We obtain $R_{N}=23.4 \mathrm{k} \Omega$ from the inverse slope of the $I-V$ characteristic at high current bias when the array was driven normal by a $0.4 \mathrm{~T}$ magnetic field. Combining $R_{N}$ with the measured value of $2 \Delta=0.35 \mathrm{meV}$ we estimate $E_{J} / k_{B} \approx 0.28 \mathrm{~K}$. From the measured junction area of $0.005 \mu \mathrm{m}^{2}$ and work of other groups [36] implying a specific capacitance of $\approx 100 \mathrm{fF} / \mu \mathrm{m}^{2}$ for junctions with similar area and resistance we estimate $C \approx 0.5 \mathrm{fF}$. We obtain a charging energy $E_{C} / k_{B} \approx 1.9 \mathrm{~K}$, so that in the absence of a ground plane $E_{C} / E_{J} \approx 6.8$, and the array would be insulating at low temperatures [36].

To measure $C_{g}$ we bias the array, driven normal with a magnetic field, with a small current (about $85 \mathrm{pA}$ ) and measure changes in the voltage across the array as a function of the voltage between it and the ground plane $V_{F G}$ (see Fig. 11.1). The array voltage is periodic in $V_{F G}$ with period $e / C_{g}$. To determine $C_{g}$ accurately, we measured the power spectrum of the oscillations; the position of the peak yielded $C_{g}=2.96 \pm 0.04 \mathrm{fF}$. We also checked for electrical isolation between the array and 2DEG while varying $V_{F G}$, and found the leakage resistance to be in excess of $10 \mathrm{G} \Omega$. Using the above values of $C$ and $C_{g}$ 


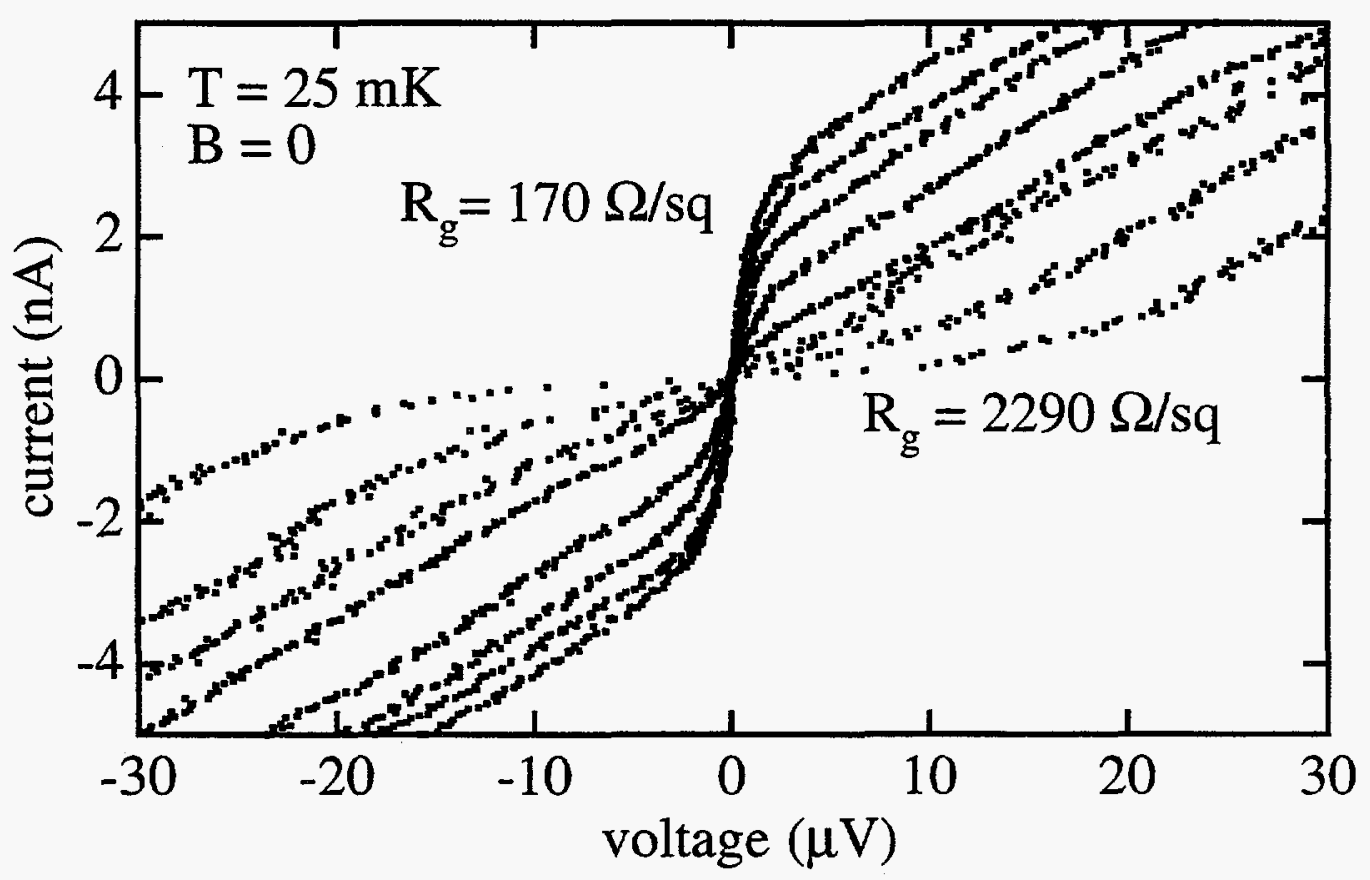

Figure 11.2: $I-V$ characteristics of the array at zero magnetic field and $T=25 \mathrm{mK}$ for eight back gate voltages $V_{B G}=0,100,200,300,400,450,500$, and $525 \mathrm{~V}$, corresponding to ground plane resistances $R_{g}=170,240,300,570,990,1350,1900$, and $2290 \Omega / \mathrm{sq}$, respectively. For $R_{g}=170 \Omega / \mathrm{sq}$, the $I-V$ characteristic is clearly superconductor-like, while for $R_{g}=2290 \Omega / \mathrm{sq}$ it has become insulator-like and shows a clear Coulomb gap. The nature of the $I-V$ characteristic can be varied continuously between these two extremes by changing $V_{B G}$.

we obtain $E_{C_{\Sigma}} / k_{B} \approx 0.68 \mathrm{~K}$. The ratio $E_{C_{\Sigma}} / E_{J} \approx 2.4$ suggests that the array may show superconducting behavior for sufficiently small $R_{g}$.

\subsection{Current-Voltage Characteristics of Array}

In Fig. 11.2 is shown the current-voltage $(I-V)$ characteristics for the array for a series of back gate voltages, at $T=25 \mathrm{mK}$ and in zero magnetic field. We define the zero-bias resistance $R_{0}$ as the inverse slope of the $I-V$ characteristic at zero current and voltage. Since the array is square, $R_{0}$ is equivalent to the resistance per square. When $V_{B G}=0 \mathrm{~V}$ and $R_{g}$ is small $(170 \Omega / \mathrm{sq})$, the $I-V$ characteristic is clearly superconductorlike, showing a small but clear "supercurrent," with low $R_{0}$. However, when we increase $R_{g}$ to $2290 \Omega / \mathrm{sq}$ by applying a large back gate voltage, the $I-V$ characteristic changes 
dramatically: the supercurrent is completely suppressed, and a pronounced charging gap appears. The $I-V$ characteristic changes smoothly between these two extremes as we increase $V_{B G}$, with the supercurrent shrinking and $R_{0}$ increasing. The transition from superconductor- to insulator-like behavior occurs over a narrow range in $V_{B G}$ of about 20 $V$. It is interesting to note that at the center of the transition, when the $I-V$ characteristic shows neither a supercurrent nor a charging gap, we measure $R_{0} \approx 6.5 \mathrm{k} \Omega / \mathrm{sq}$. This is consistent with recent theories [31,33,94] which predict a universal value on the order of $R_{Q}$ for the zero-temperature resistance of a system of charge- $2 e$ bosons at the $\mathrm{S}-\mathrm{I}$ transition.

\subsection{Effects of Varying Ground Plane Resistance on Param- eters of Array}

Applying a back gate voltage clearly cannot change the junction capacitance. However, it is conceivable that varying $V_{B G}$ could change $C_{g}$, either due to motion of the center of mass of the 2DEG, or in the case of extreme depletion due to breakup of the 2DEG itself. To investigate this possibility, we measured $C_{g}$ for several different values of $V_{B G}$, to a maximum of $500 \mathrm{~V}$. To within the experimental accuracy of our measurement $( \pm 0.04 \mathrm{fF})$, there was no change in the value of $C_{g}$. We also verified that the tunneling resistance $R_{N}$ was independent of $V_{B G}$. As mentioned above, the $2 \mathrm{DEG}$ is not expected to show significant non-uniformity in the range of $n_{s}$ covered in our experiment. We estimate the average number of electrons in the $2 D E G$ per unit cell, with an area of $4.7 \mu \mathrm{m}^{2}$, decreases from about 9600 to 3300 as $V_{B G}$ changes from $0 \mathrm{~V}$ to $540 \mathrm{~V}$, so that fluctuations in the number of screening electrons per unit cell are at worst about $2 \%$. The fact that $C_{g}$ is independent of $V_{B G}$ also argues against any breakup of the 2DEG. Finally, when we measure the $I-V$ characteristics with the islands in the superconducting state, the array and $2 \mathrm{DEG}$ are electrically connected, so that $V_{B G}$ induces electric fields only between the back gate and the 2DEG. We conclude that applying a back gate voltage changes only $R_{g}$ of the ground plane, that is, the dissipative electrodynamic environment of the array, and no other relevant physical parameters. 


\subsection{Zero-Bias Resistance of Array}

Plotting $R_{0}$ versus $R_{g}$ on semi-log axes as shown in Fig. 11.3, we see that $R_{0}$ increases exponentially with $R_{g}$. We show $R_{0}$ versus $R_{g}$ (markers) and fits (solid lines) of the data to the form $R_{0}=R_{1} \exp \left(R_{g} / R_{2}\right)$ at $25,50,100$, and $150 \mathrm{mK}$. At $T=25 \mathrm{mK}$, the array is extraordinarily sensitive to $R_{g}$ : increasing $R_{g}$ by a factor of 16 leads to an increase in $R_{0}$ of over 2 orders of magnitude. This extreme sensitivity diminishes at higher temperatures, until at $150 \mathrm{mK} R_{0}$ depends only weakly on $R_{g}$. The values of the fitting parameters are $R_{1}=272,162,205$, and $1390 \Omega /$ sq and $R_{2}=443,556,913$, and $1830 \Omega / \mathrm{sq}$ for the four temperatures above, respectively.

The inset to Fig. 11.3 shows the temperature dependence of $R_{0}$ for $R_{g}=170 \Omega / \mathrm{sq}$ and $R_{g}=2290 \Omega / \mathrm{sq}$. At low temperatures, for $R_{g}=2290 \Omega / \mathrm{sq}$ the array is clearly insulating with $R_{0}$ increasing by 2 orders of magnitude as $T$ decreases from 100 to $25 \mathrm{mK}$. For $R_{g}=170 \Omega / \mathrm{sq}$, on the other hand, $R_{0}$ drops rapidly with decreasing temperature until it reaches a minimum value at approximately $50 \mathrm{mK}$, below which it rises again. As $T$ tends to zero, $R_{0}$ appears to approach a constant value: we measure roughly the same value for $R_{0}\left(R_{g}=170 \Omega / \mathrm{sq}\right)$ at both 37 and $25 \mathrm{mK}$. We cannot rule out sample heating as the cause of this behavior; a sample with a lower $R_{N}$ would be required to investigate this issue. The quasi-reentrant nature of the superconductivity in the present work suggests that our array would not reach a zero-resistance state at $T=0 \mathrm{~K}$. Similar quasi-reentrant behavior has been seen in previous studies of array [17] and granular films [67,66,46], and is the subject of some debate $[51,45]$.

\subsection{Comparison of Results to Theory}

The results of our experiment prompted Wagenblast et al. [93] to perform a theoretical study of our system, a 2D array of Josephson junctions capacitively coupled to a 2DEG, as described in Chap. 5. Shown in Fig. 11.4 are the predicted zero-bias resistance $R_{0}$, in units of the resistance quantum $R_{Q}$, versus temperature data for different values of ground plane resistance $R_{g}$, including a parallel thermally activated channel, for an array with $e^{2} / C_{g}=0.2 \mathrm{~K}, E_{J}=0.28 \mathrm{~K}, C_{g} / C=10$, and $R_{N}=23 \mathrm{k} \Omega$. These values are similar to those of our array with $e^{2} / C_{g} \approx 0.63 \mathrm{~K}, E_{J} \approx 0.28 \mathrm{~K}, C_{g} / C \approx 6$, and $R_{N}=23.4 \mathrm{k} \Omega$. The data are shown for ground plane resistances $R_{g}=200,700,1200,1700,2200$, and 


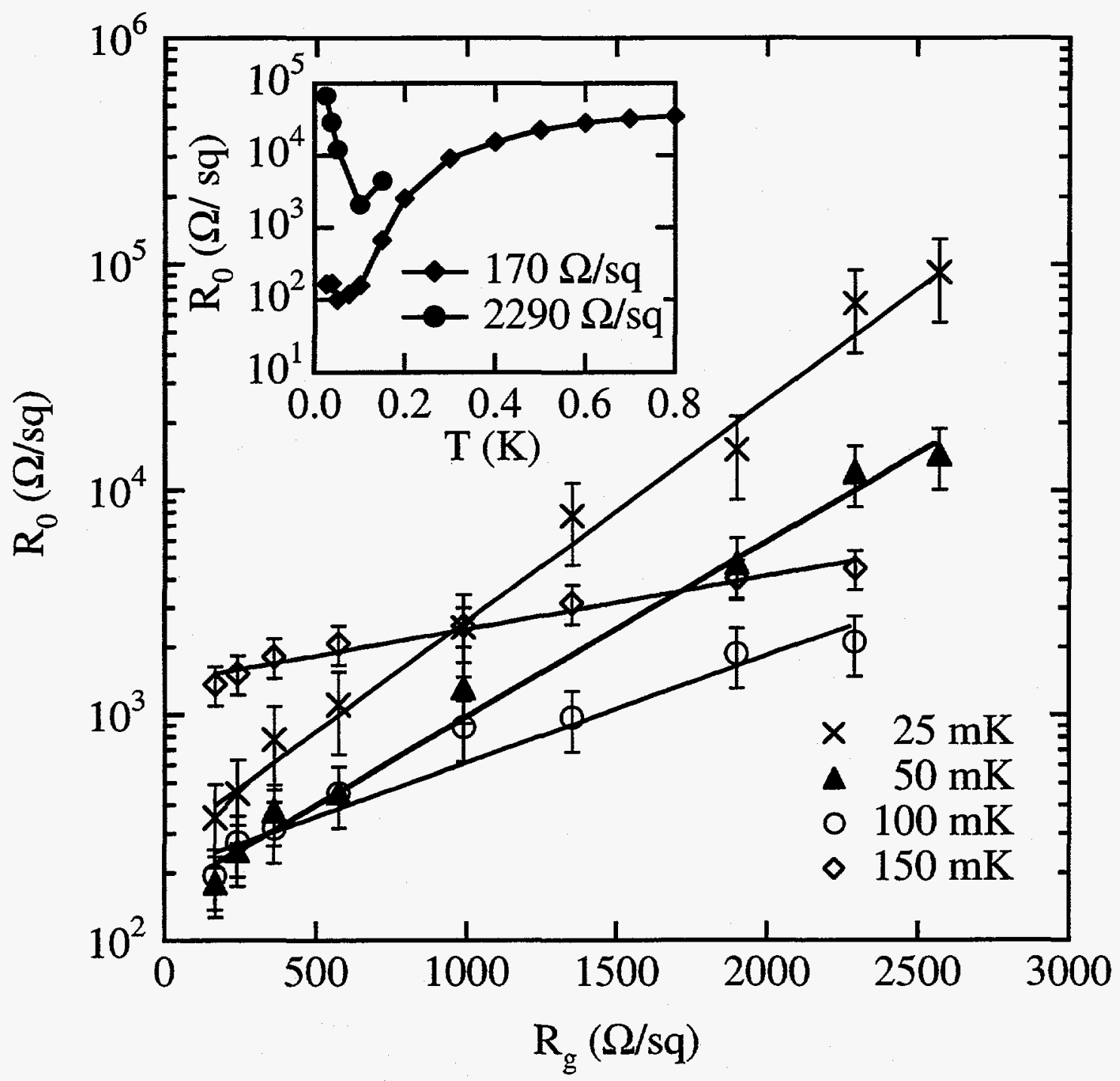

Figure 11.3: Zero-bias resistance $R_{0}$ of the array versus the resistance per square $R_{g}$ of the ground plane for four temperatures $T=25,50,100$, and $150 \mathrm{mK}$. The solid lines are fits to an exponential as described in the text. The inset shows the temperature dependence of $R_{0}$ for $R_{g}=170 \Omega / \mathrm{sq}$ and $2290 \Omega / \mathrm{sq}$. 


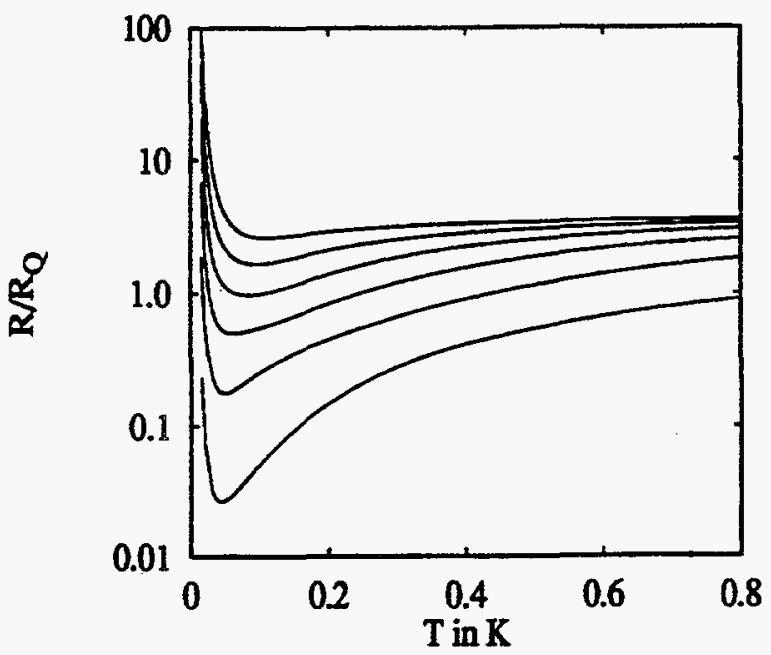

Figure 11.4: Predictions of Wagenblast et al. for the zero-bias resistance of an array, in units of the resistance quantum $R_{Q}$, versus temperature for six different values of the ground plane resistance $R_{g}=200,700,1200,1700,2200$, and $2700 \Omega /$ sq. The array parameters are $e^{2} / C_{g}=0.2 \mathrm{~K}, E_{J}=0.28 \mathrm{~K}, C_{g} / C=10$, and $R_{N}=23 \mathrm{k} \Omega$. A parallel thermally activated channel $R_{N} \exp \left(E_{a} / T\right)$ with $E_{a}=0.2 \mathrm{~K}$ is included.

$2700 \Omega$ /sq. The data resemble the data measured in our experiment (see Fig. 11.3). Most notably, the predicted curves show the quasi-reentrant behavior we see at small values of $R_{g}$, with $R_{0}$ reaching a minimum and then increasing again at very low temperatures. Shown in Fig. 11.5 is the data from Fig. 11.4 in the form of $\log R_{0}$ versus $R_{g}$ for different temperatures. Comparing these curves to those of ours in Fig. 11.3, we again see a nice similarity.

We compare our experimental results to the phase diagram of Wagenblast et al. in Fig. 11.6, showing the superconducting and insulating phases in the $E_{J} / E_{C}$ versus $\alpha=R_{Q} / R_{g}$ plane. According to this diagram, we have observed a transition from one insulating state to another, with the zero-bias resistance of the array versus temperature changing from Fig. 11.6 (d) to (c) as the ground plane resistance is increased. Therefore, we should be able to observe the quasi-critical dissipation-driven superconductor-insulator transition behavior described by Wagenblast et al. (transition from Fig. 11.6 (b) to (c)) by measuring a sample with a lower ratio of $E_{J} / E_{C}$. In the strict sense, a true dissipationdriven superconductor-insulator transition can only observed at $T=0 \mathrm{~K}$, which is inaccessible in our experiments. 


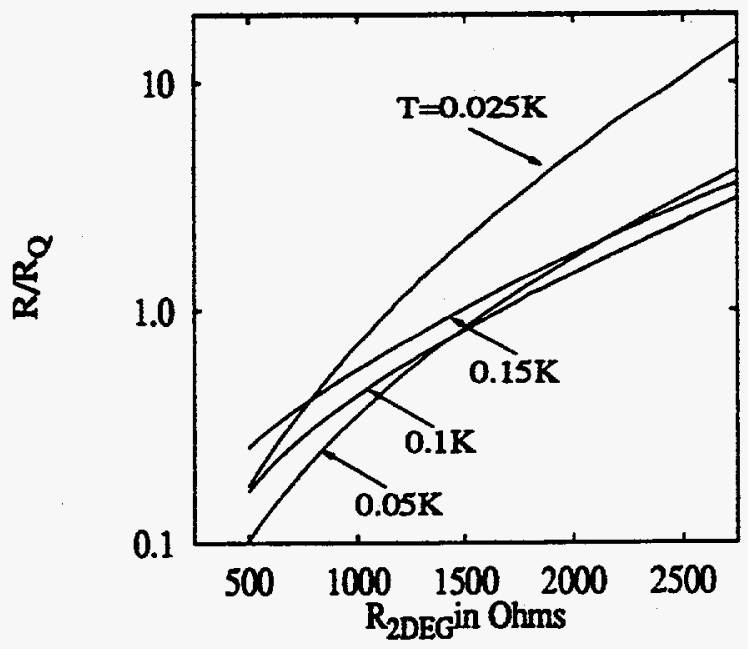

Figure 11.5: Predictions of Wagenblast et al. for the zero-bias resistance of the array described in Fig. 11.4, in units of the resistance quantum $R_{Q}$, versus ground plane resistance $R_{g}\left(R_{g} \equiv R_{2 D E G}\right)$ for four different temperatures $T=25,50,100$, and $150 \mathrm{mK}$.

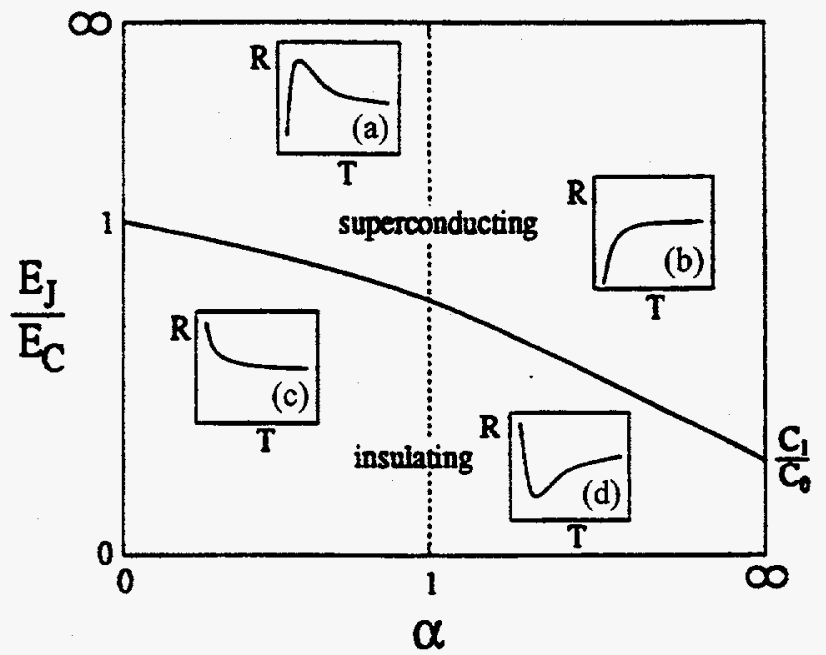

Figure 11.6: Phase diagram from Wagenblast et al. for an array capacitively coupled to a 2DEG. The superconducting and insulating phases in the $E_{J} / E_{C}$ versus $\alpha=R_{Q} / R_{g}$ plane are shown. The insets (a)-(d) show the zero-bias resistance of the array as a function of temperature in the different regions. 


\subsection{Conclusion}

In conclusion, we have presented measurements of the $I-V$ characteristics of a $2 \mathrm{D}$ array of superconducting islands linked by Josephson junctions and in close proximity to a $2 \mathrm{DEG}$. We use the $2 \mathrm{DEG}$ as a source of variable dissipation for the array by varying its resistance per square $R_{g}$ via a back gate voltage $V_{B G}$. Measurements of the capacitance to ground $C_{g}$ of the islands indicate it is independent of $V_{B G}$, so that changing $V_{B G}$ changes only the dissipation and no other relevant physical parameters. As the dissipation is decreased, the $I-V$ characteristics of the array change from superconductor-like with a pronounced supercurrent to insulator-like with a clear charging gap. Simultaneously the zero-bias resistance increases exponentially with $R_{g}$. We interpret these data as evidence for a dissipation-driven transition. The theoretical model of Wagenblast et al. is in reasonable agreement with our data. Many questions remain unanswered, such as the effect of the dimensionality of the array on the transition and to what universality class the transition belongs. 


\section{Chapter 12}

\section{Magnetic Field Dependence of a Dissipation-Driven}

\section{Superconductor-Insulator}

\section{Transition in a Two-Dimensional}

\section{Array of Josephson Junctions}

\subsection{Introduction}

A variety of two-dimensional (2D) superconducting systems undergo a superconductor-to-insulator (S-I) transition as the applied magnetic field is increased. Examples of such systems are superconducting thin films [41,81] and $2 \mathrm{D}$ arrays of Josephson junctions $[87,18]$. The 2D S-I transition has also been seen in highly anisotropic threedimensional superconductors [77]. A scaling theory for this magnetic field-driven transition is given by Fisher [33] (see Chap. 5). Here we investigate the magnetic field-driven S-I transition in a $2 \mathrm{D}$ array of Josephson junctions over a ground plane with variable resistance, as described in Chap. 11. By varying the resistance of the ground plane, we vary the dissipation associated with the local electrodynamic environment of the system. The dissipation-driven S-I transition observed at zero applied magnetic field is described in Chap. 11. In this chapter is described the effects of changing both the applied magnetic 
field and dissipation associated with the electrodynamic environment of the system simultaneously.

\subsection{Description of Sample}

The sample is a $2 \mathrm{D}$ array of submicron Josephson junctions over a ground plane, where the resistance of the ground plane, and thus the dissipation in the electrodynamic environment, can be varied continuously in situ. The sample is described in more detail in Chap 11. The array is a $40 \times 402 \mathrm{D}$ lattice of $\mathrm{Al}$ islands separated by approximately $70 \times 70 \mathrm{~nm}^{2} \mathrm{Al} / \mathrm{Al}_{\mathrm{x}} \mathrm{O}_{\mathbf{y}} / \mathrm{Al}$ tunnel junctions. The array is fabricated on a $\mathrm{GaAs} / \mathrm{Al}_{0.3} \mathrm{Ga}_{0.7} \mathrm{As}$ heterostructure with a $2 \mathrm{D}$ electron gas (2DEG) located approximately $100 \mathrm{~nm}$ below the surface. The resistance of the $2 \mathrm{DEG}$ can be varied continuously in the range $R_{g} \approx 170-$ $2290 \Omega$ /sq by applying a large voltage $V_{B G}$ between the $2 \mathrm{DEG}$ and a back gate.

\subsection{Description of Experiment}

Measurements are performed on the sample in a dilution refrigerator at temperatures $T \geq 20 \mathrm{mK}$. Details of the experimental configuration are given in Chap. 11 . We apply a small magnetic field (up to $\approx 5 \times 10^{-4} \mathrm{~T}$ ) perpendicularly to the array. The magnetic field is measured in units of frustration, where frustration $f$ is defined as the number of flux quanta $\Phi_{0}=2.07 \times 10^{-15} \mathrm{Tm}^{2}$ per unit cell area. Details of the magnetic field dependent measurement configuration is given in Chapter 8 .

\subsection{Current-Voltage Characteristics of Array at Smallest Ground Plane Resistance}

Shown in Fig. 12.1 are the current-voltage $(I-V)$ characteristics of the 2D array for a series of frustrations ranging from $f=0$ to 0.37 , at $V_{B G}=0$ corresponding to $R_{g}=170$ $\Omega / \mathrm{sq}$, at the base temperature of the dilution refrigerator $\approx T=20 \mathrm{mK}$. At frustration $f$ $=0$, the $I-V$ characteristic is clearly superconductor-like, with a supercurrent-like feature approximately $20 \mathrm{nA}$ in size. As the frustration is increased, the $I-V$ characteristic is no longer superconductor-like, developing a slope that increases with increasing $f$. At $f=$ 0.05 , the $I-V$ characteristic becomes insulator-like, with a a small Coulomb gap appearing. 


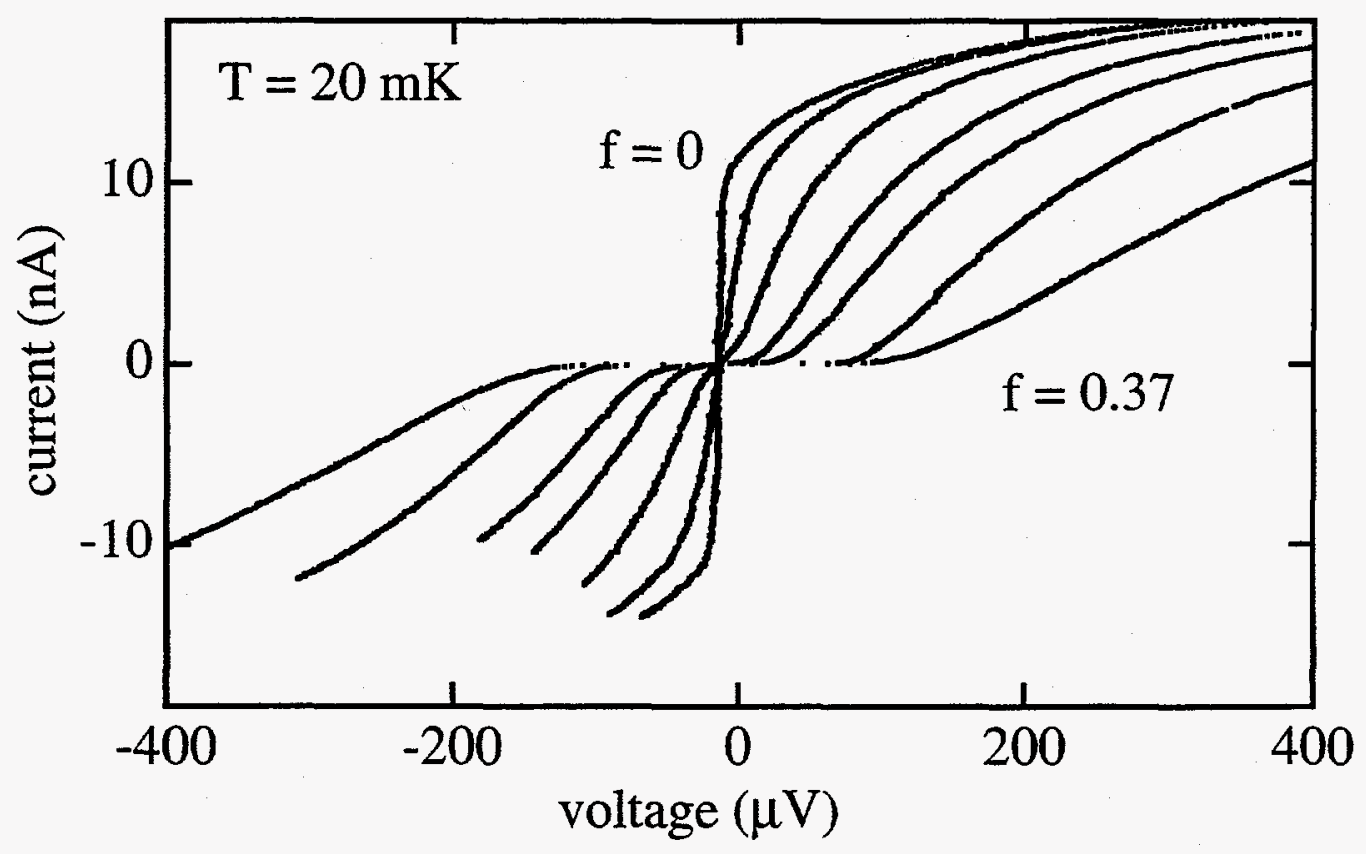

Figure 12.1: Current-voltage characteristics of the array at ground plane resistance $R_{g}=$ $170 \Omega / \mathrm{sq}$ and $T=20 \mathrm{mK}$ for seven different frustrations $f=0,0.007,0.025,0.05,0.1,0.2$, and 0.37 . For $f=0$, the $I-V$ characteristic is superconductor-like, while at $f=0.37$ it is insulator-like. 


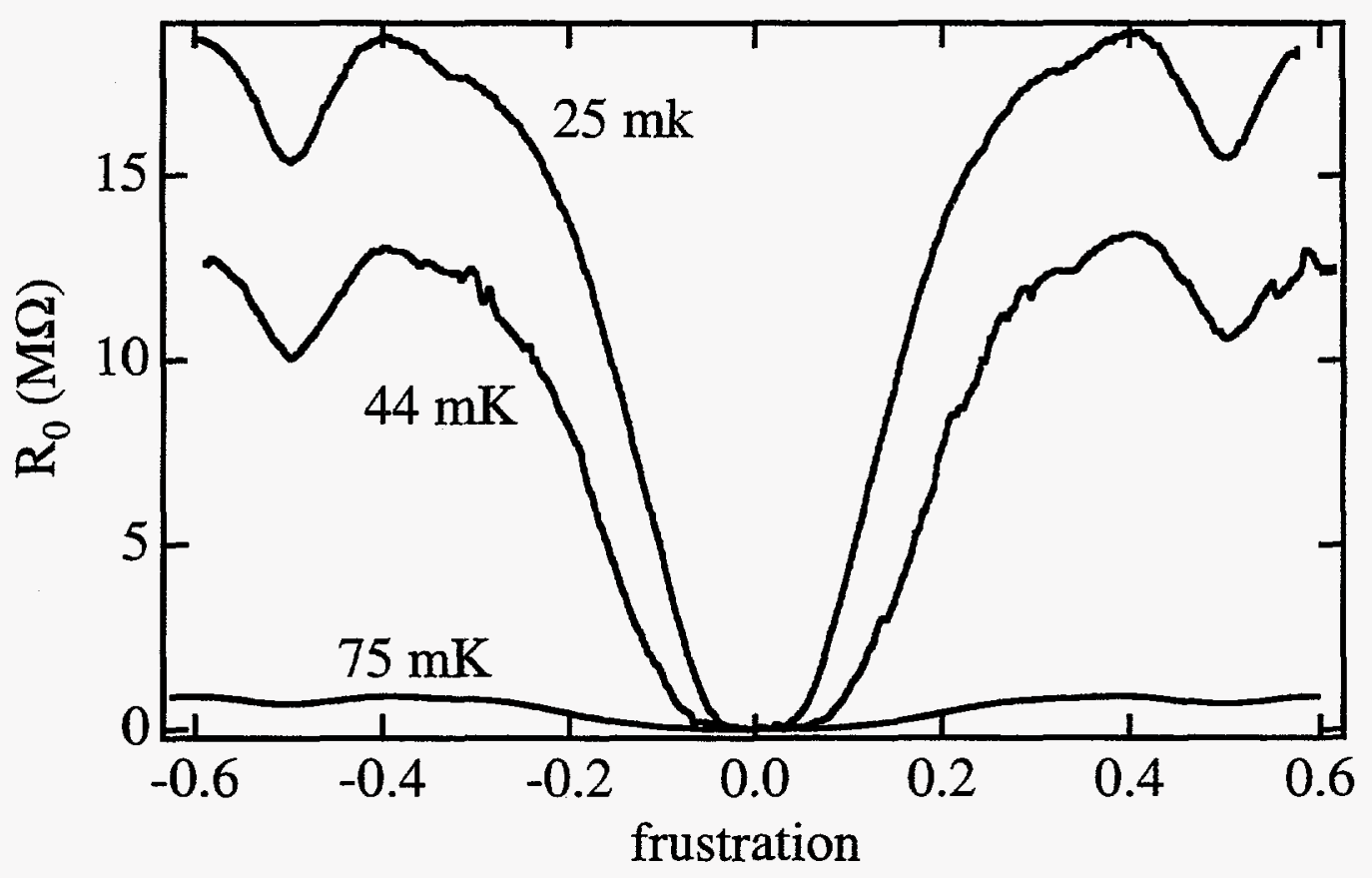

Figure 12.2: Zero-bias resistance $R_{0}$ of the array versus frustration $f$ at the smallest value of resistance per square of the ground plane $R_{g}=170 \Omega / \mathrm{sq}$ for three temperatures $T=25$, 44 , and $75 \mathrm{mK}$.

As the frustration is increased further, the size of the Coulomb gap increases. At $f=0.37$, the gap is approximately $350 \mu \mathrm{V}$ in size. Clearly, the $I-V$ characteristics of the array change from superconductor-like to insulator-like as the frustration is increased.

\subsection{Zero-Bias Resistance of Array at Smallest Ground Plane Resistance}

Shown in Fig. 12.2 are plots of the zero-bias resistance $R_{0}$ of the array versus frustration $f$ for temperatures $T=25,44$, and $75 \mathrm{mK}$ at the smallest value of resistance per square of the ground plane $R_{g}=170 \Omega / \mathrm{sq}$. As expected, the curves have a minimum at $f=0$ and a local minimum at $f=1 / 2$. The energy, and thus the resistance, of the system is lowered at these values of $f$ since the vortex lattice is commensurate with the array lattice. The change in $R_{0}$ as a function of frustration is greatest at the lowest temperature.

The $I-V$ characteristics of the array indicate that we observe a magnetic fielddriven superconductor-insulator transition. We also determine if the temperature depen- 


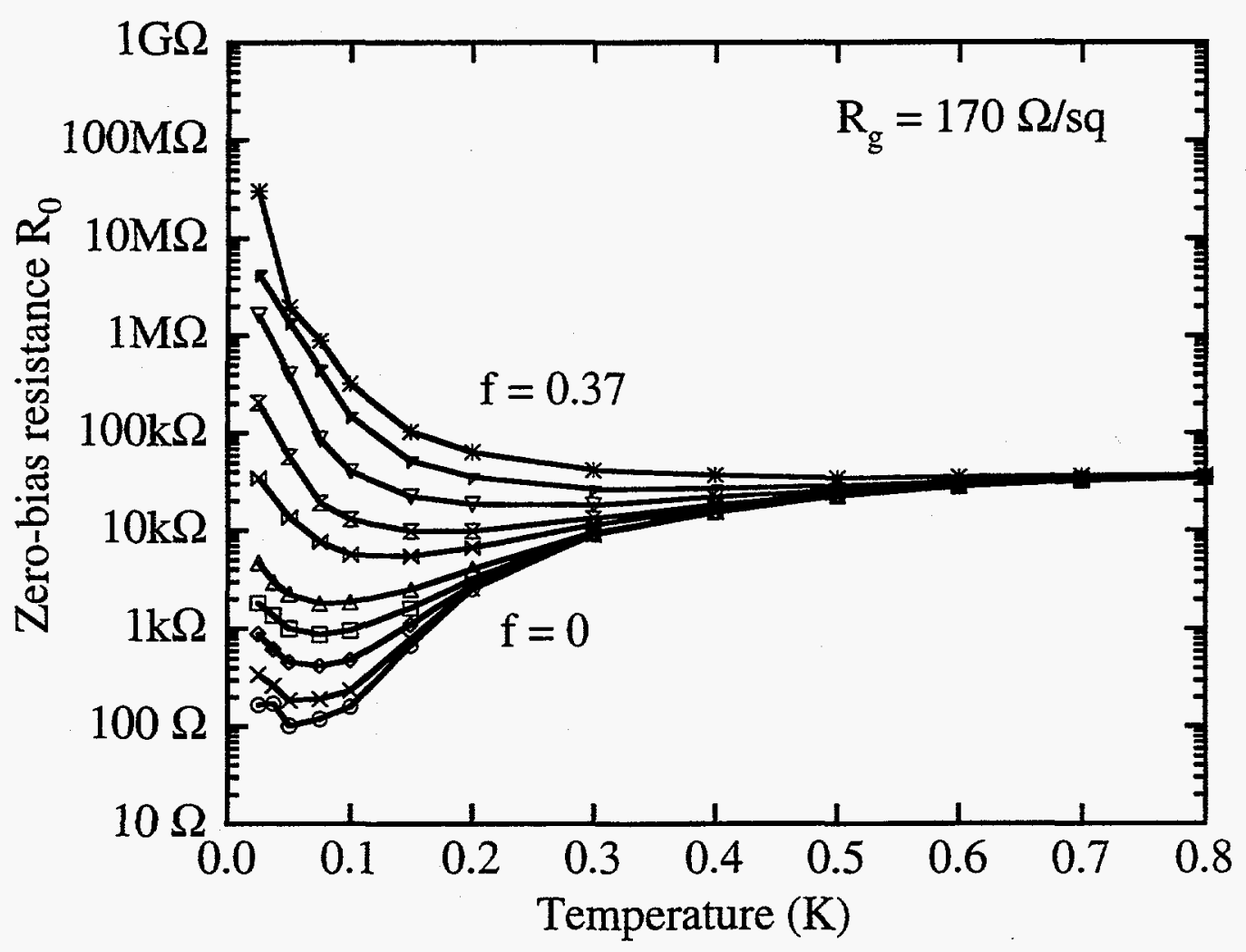

Figure 12.3: Zero-bias resistance $R_{0}$ of the array versus temperature for ten different frustrations $f=0,0.001,0.0025,0.005,0.01,0.025,0.05,0.1,0.2$, and 0.37 , from bottom to top, at resistance per square of the ground plane $R_{g}=170 \Omega / \mathrm{sq}$.

dence of the zero-bias resistance $R_{0}$ indicates a B-driven S-I transition. Shown in Fig. 12.3 is $\log R_{0}$ versus temperature $T$ for a series of different frustrations ranging from $f=0$ to 0.37 at the smallest ground plane resistance $R_{g}=170 \Omega /$ sq. At frustration $f=0, R_{0}$ drops rapidly with decreasing temperature until it reaches a minimum value at approximately 50 $\mathrm{mK}$, below which it rises again. As discussed in Chap. 11, the origin of this behavior is not altogether clear. The theory of Wagenblast et al. [93] explains this behavior as the phase of the array undergoing a transition from dissipative to capacitive dynamics. However, we cannot rule out sample heating in our experiment.

As the frustration is increased, $R_{0}$ decreases less with decreasing $T$, but the quasireentrant behavior is still evident. Between $f=0.01$ and $0.05, R_{0}$ as a function of $T$ makes a transition from decreasing with decreasing $T$ to increasing with decreasing $T$ for $T \leq 150 \mathrm{mK}$; at $f=0.05, R_{0}$ versus $T$ shows insulating behavior, with $R_{0}$ increasing 
steadily with decreasing $T$. As $f$ is increased further, the increase of $R_{0}$ with decreasing $T$ increases. Thus, the $R_{0}$ versus $T$ characteristics of the array show a transition from superconductor-like to insulator as the magnetic field is increased. This transition is similar to that seen by Chen et al. [18] in their work with 2D Josephson junction arrays with no ground plane. As they increase the frustration, the $R_{0}$ versus $T$ curves of their array also make a transition from being superconductor-like with quasi-reentrant behavior to insulating; in contrast to our work, at the lowest frustrations, the $R_{0}$ versus $T$ ' curves of their array are superconductor-like with no quasi-reentrant behavior.

\subsection{Zero-Bias Resistance of Array at Different Values of Ground Plane Resistance}

We investigate the effects of changing the dissipation in the system, by changing the back gate voltage $V_{B G}$ and thus the resistance of the ground plane $R_{g}$, on the magnetic field dependence of the zero-bias resistance $R_{0}$ of the array. Shown in Fig. 12.4 are small-range frustration curves ( $R_{0}$ versus $f$ ) for values of $R_{g}$ ranging from $R_{g}=170$ to $1350 \Omega / \mathrm{sq}$. Over the range of frustration shown, $f=-0.015$ to 0.015 , the zero-bias resistance $R_{0}$ increases as the ground plane resistance $R_{g}$ is increased.

We also plot $R_{0}$ as a function of $f$ and $R_{g}$ in a different manner to investigate the functional dependence of $R_{0}$ on $R_{g}$. Shown in Fig. 12.5 is $\log R_{0}$ versus $R_{g}$ for four different values of frustration ranging from $f=0$ to 0.006 . Over the limited range of $f$ shown, $R_{0}$ increases exponentially with ground plane resistance $R_{g}$. Shown is $R_{0}$ versus $R_{g}$ (markers) and fits (solid lines) of the data to the form $R_{0}=R_{1} \exp \left(R_{g} / R_{2}\right)$. The dependence of $R_{0}$ on $R_{g}$ decreases as $f$ is increased. The values of the fitting parameters are $R_{1}=140,600,1280$, and $2150 \Omega$ and $R_{2}=410,570,620$, and $660 \Omega /$ sq for the frustrations $f=0,0.002,0.004$, and 0.006 , respectively.

\subsection{Ground Plane Resistance Versus Frustration Phase Di- agram}

As discussed in Chapter 11, the $I-V$ characteristics of the $2 \mathrm{D}$ array with variable dissipation make a transition from being superconductor-like to insulator-like at frustration 


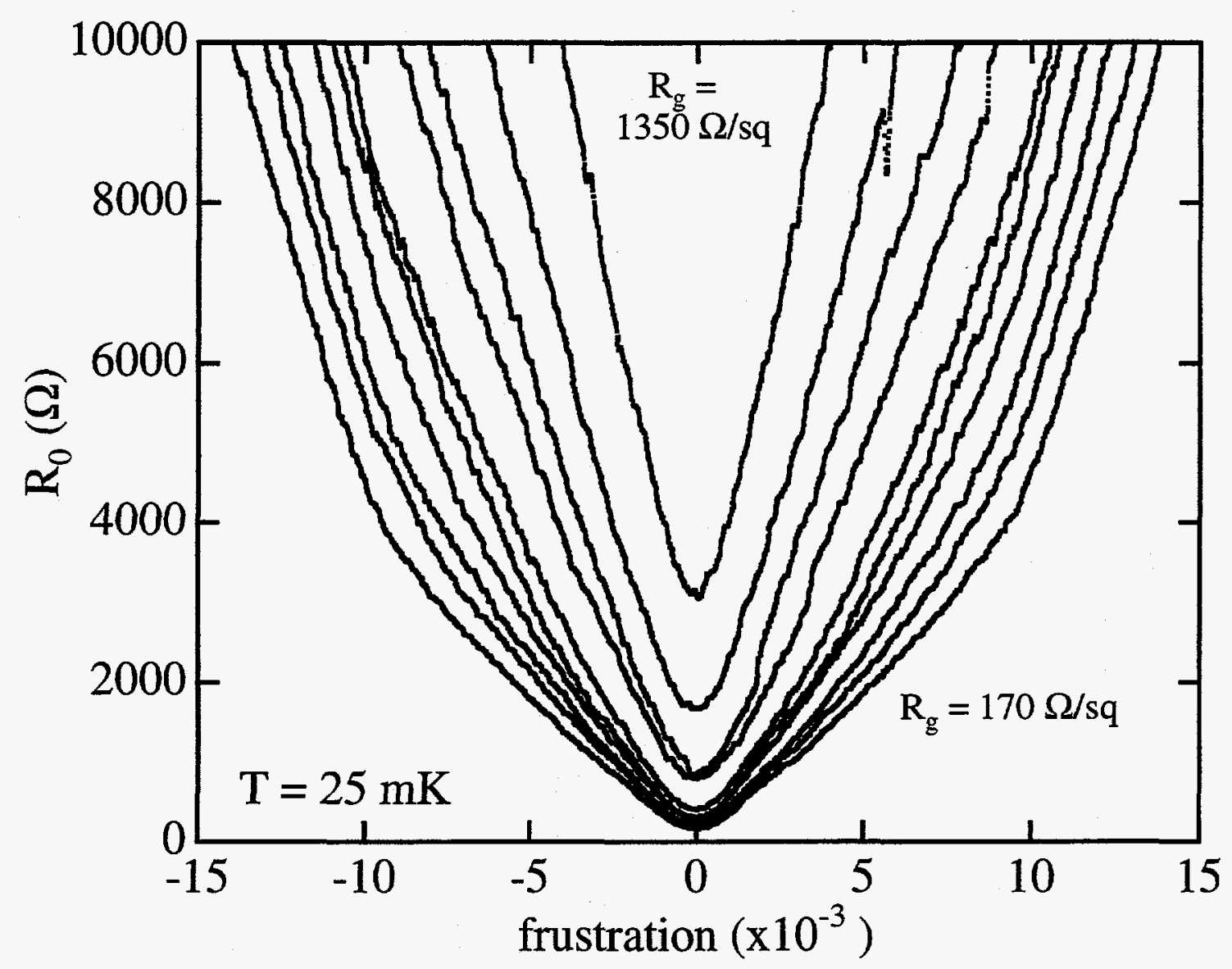

Figure 12.4: Zero-bias resistance $R_{0}$ of the array versus frustration $f$ for ten different values of the ground plane resistance $R_{g}=170,200,240,290,360,450,570,740,990$, and 1350 $\Omega / \mathrm{sq}$, from bottom to top. 


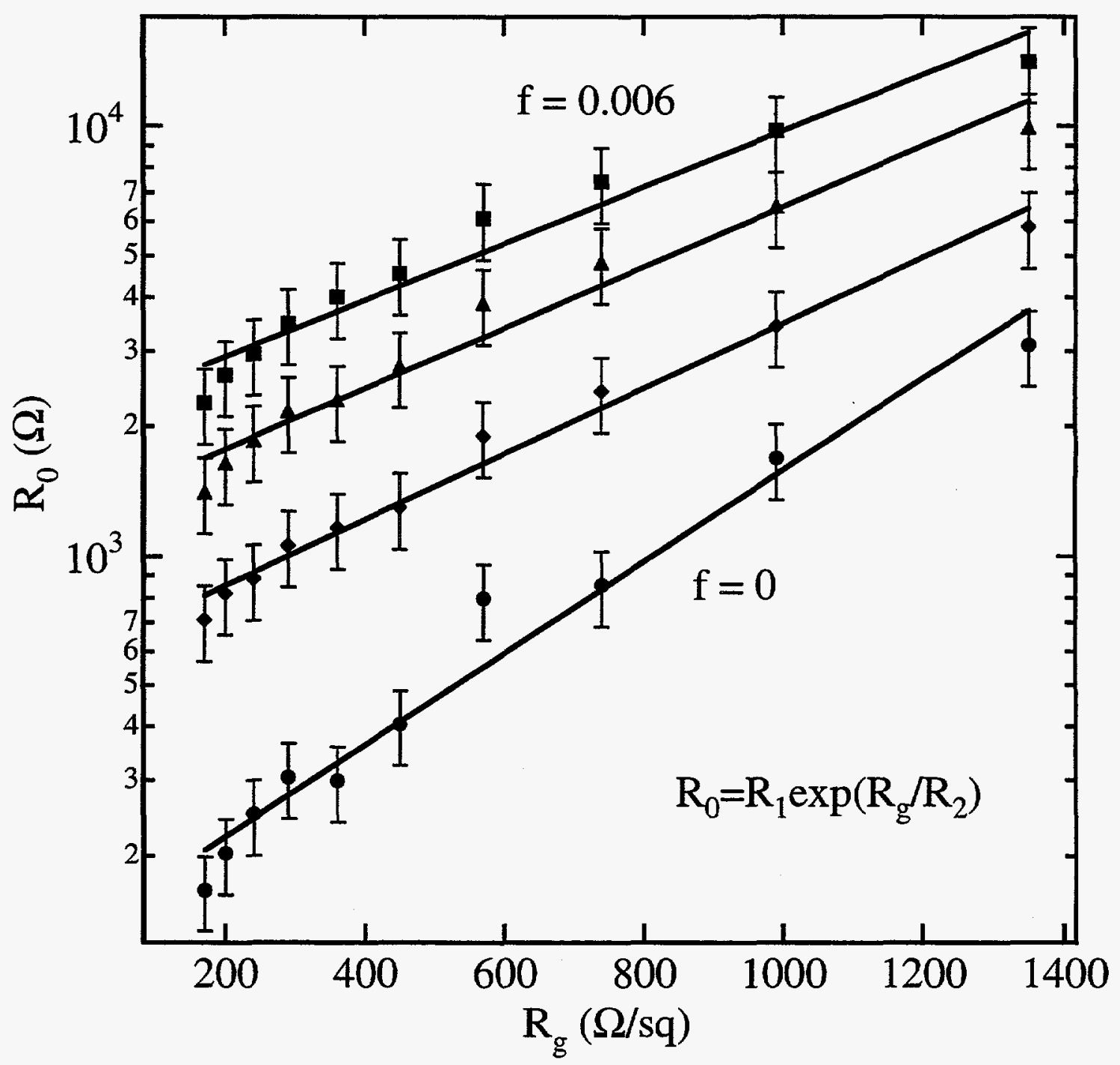

Figure 12.5: Zero-bias resistance $R_{0}$ of the array versus the resistance per square of the ground plane $R_{g}$ for four frustrations $f=0,0.002,0.004$, and 0.006 , from bottom to top. The solid lines are fits to an exponential as described in the text. 


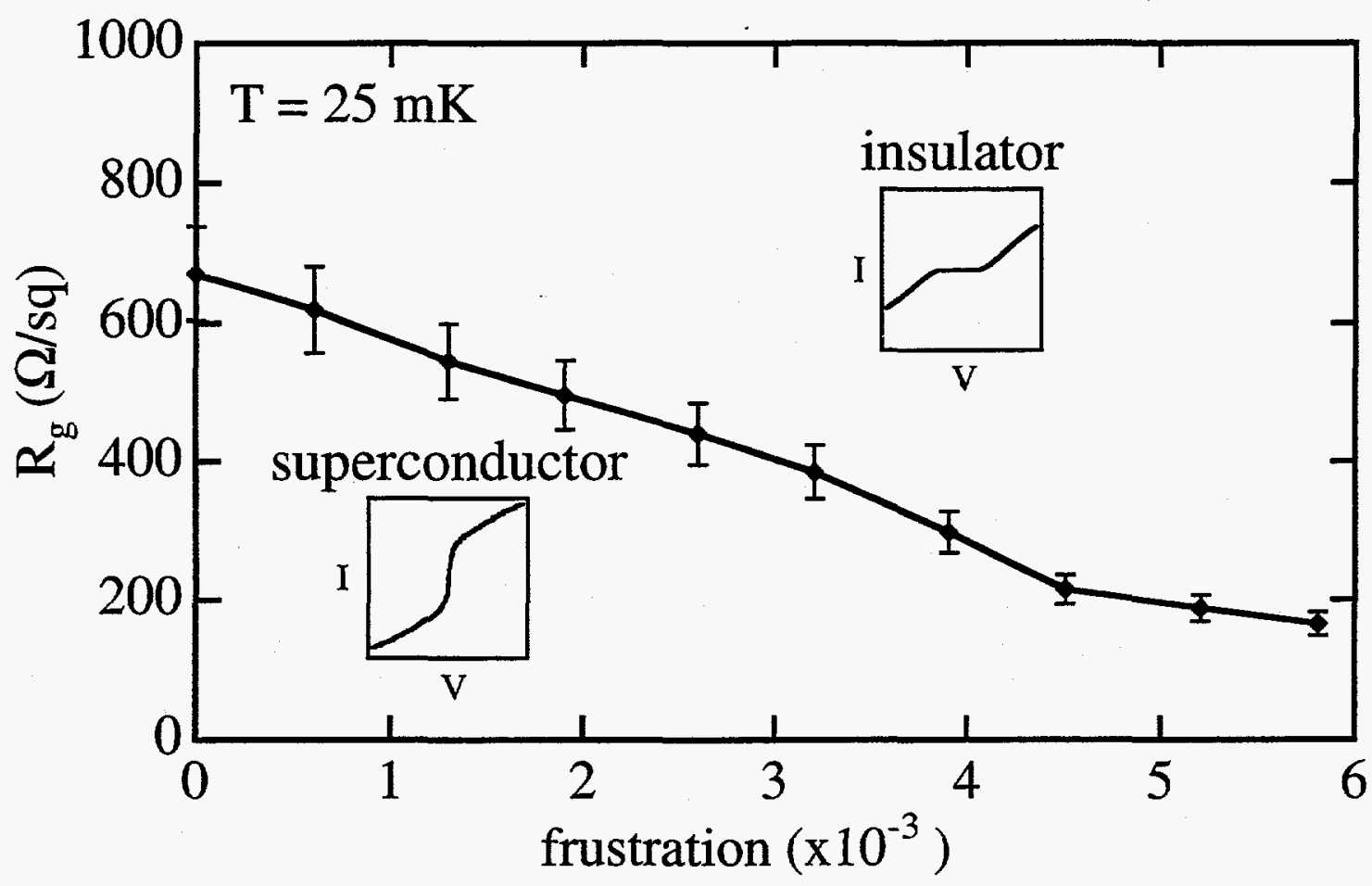

Figure 12.6: Phase diagram for the superconductor-like to insulator-like transition of the $I-V$ characteristics of the array in the ground plane resistance $R_{g}$ versus frustration $f$ plane.

$\mathrm{f}=0$ as the ground plane resistance $R_{g}$ is increased. A similar transition occurs as the frustration $f$ is increased and $R_{g}$ is held constant, as discussed in the previous sections. In this section is discussed the behavior of this transition when both $f$ and $R_{g}$ are varied simultaneously. Shown in Fig. 12.6 is the phase diagram for the superconductor-like to insulator-like transition of the $I-V$ characteristics of the array in the $R_{g}$ versus $f$ plane at $T=25 \mathrm{mK}$. The markers indicate the value of $R_{g}$ at which the $I-V$ characteristic of the array makes a transition from superconductor-like to insulator-like, showing neither a supercurrent nor a charging gap. The transition value of $R_{g}$ is determined by holding the frustration $f$ constant and then measuring the $I-V$ characteristics at different values of the ground plane resistance $R_{g}$. At the transition point, the $I-V$ characteristic is linear, with a zero-bias resistance close to the resistance quantum for Cooper pairs $R_{Q} \approx 6.45 \mathrm{k} \Omega$ (the critical values of $R_{0}$ range from 6.6 to $7.9 \mathrm{k} \Omega$ ). The line connecting the markers are an aid to guide the eye. Below the line, the $I-V$ characteristic of the array is superconductor-like, 
with a supercurrent-like feature. Above the line, the $I-V$ characteristic is insulator-like, with a Coulomb gap. In general, the superconductor-like to insulator-like transition of the $I-V$ characteristics occurs at lower values of $R_{g}$ for higher values of $f$. This behavior is reasonable, since the $I-V$ characteristics become more insulating with increasing $R_{g}$ or $f$, so the transition should occur at decreasing values of $R_{g}$ for increasing values of $f$.

\subsection{Scaling Behavior of the Magnetic Field-Driven Super- conductor-Insulator Transition at the Smallest Ground Plane Resistance}

Our results can be compared to the scaling theory of Fisher [33] for the magnetic field-driven S-I transition in 2D superconducting systems as described in Chapter 5 . In this theory, the resistivity $\rho$ of the system near the critical field $B_{c}$ should be given by the scaling form:

$$
\rho(B, T)=\frac{h}{4 e^{2}} \tilde{\rho}\left[\frac{c_{0}\left(B-B_{c}\right)}{T^{1 / z_{B} \nu_{B}}}\right]
$$

where $c_{0}$ is a nonuniversal constant, $z_{B}$ is the dynamical critical exponent, $\nu_{B}$ is the static critical exponent for the superconducting correlation length, and $\tilde{\rho}$ is a dimensionless scaling function. The zero-bias resistance $R_{0}$ of Josephson junction arrays described by this form should follow the expression:

$$
\frac{\mathrm{d} R_{0}}{\mathrm{~d} f} \mid f_{\mathrm{c}} \sim T^{-1 / z_{B} \nu_{B}}
$$

where $f_{c}$ is the critical frustration. Various groups compare this scaling theory to their experimental results on superconducting thin films and three-dimensional superconductors $[41,77,81]$. They determine the scaling exponents $z_{B}$ and $\nu_{B}$ by first determining the critical field $B_{c}$ from resistivity $\rho$ versus temperature $T$ data and then fitting a line to the data in a plot of $(\mathrm{d} \rho / \mathrm{d} B) \mid B_{\mathrm{c}}$ versus $T$. Determining the critical frustration $f_{\mathrm{c}}$ for arrays of Josephson junctions is not as straightforward, since the $R_{0}$ versus $T$ curves are quasi-reentrant. We also cannot use the method employed by van der Zant et al. [87] in their work with $2 \mathrm{D}$ arrays, where $f_{c}$ is identified as the point where the frustration curves for different temperatures cross and the concavity of the curves change, since our frustration curves do not cross at a single point. In our analysis, we first study $R_{0}$ versus $T$ data to determine a reasonable range of critical frustrations $f_{c}$. We then determine which $f_{c}$ from the determined range gives the straightest line when plotting $\left(\mathrm{d} R_{0} / \mathrm{d} f\right) \mid f_{c}$ versus $T$. We 
only fit the range from 75 to $400 \mathrm{mK}$ (below $75 \mathrm{mK} R_{0}$ shows quasi-reentrant behavior not consistent with the scaling theory). Using this method, we find the critical field and exponent product to be $f_{c}=0.018 \pm 0.006$ and $z_{B} \nu_{B}=0.83 \pm 0.18$ as shown in Fig. 12.7 (a). Other work with $2 \mathrm{D}$ Josephson junction arrays $[87,18]$ report values of $z_{B} \nu_{B}$ ranging from 1.5 to 8.2 and a critical resistance $R_{\text {crit }}$ (zero-bias resistance of the array at the field-driven S-I transition) ranging from 1.2 to $4 \mathrm{k} \Omega$. The critical resistance for our array is $R_{\text {crit }} \approx 7 \mathrm{k} \Omega$. For work on superconducting thin films and three-dimensional superconductors $[41,77,81]$, values of $z_{B} \nu_{B}=1.2$ to 1.4 and $R_{c r i t}=4.5$ to $13.6 \mathrm{k} \Omega$ are found. Our value of $R_{c r i t}$ is close to those found in the work with arrays and films, but our value of $z_{B} \nu_{B}$ does not agree with these works and contradicts the scaling theory [33] which predicts $z_{B} \nu_{B} \geq 1$. Shown in Fig. 12.7 (b) is the collapse of the $T=75$ to $400 \mathrm{mK}$ data for $R_{0}$ versus $\left|f-f_{c}\right| / T^{1 / z_{B} \nu_{B}}$ using the determined values of $f_{c}$ and $z_{B} \nu_{B}$. Attempting to find a better data collapse using different values of $f_{c}$ and $z_{B} \nu_{B}$ was unsuccessful.

\subsection{Conclusion}

In conclusion, we have described a $2 \mathrm{D}$ array of superconducting islands linked by Josephson junctions over a 2DEG in which both the dissipation and applied magnetic field can be varied simultaneously and in a controlled manner. The array shows a superconductor-like to insulator-like transition in the $I-V$ characteristics as the frustration is increased, for all the values of ground plane resistance $R_{g}$ investigated. The transition occurs at decreasing values of frustration as the ground plane resistance is increased. The zero-bias resistance $R_{0}$ of the array increases exponentially with $R_{g}$, with the dependence decreasing as the frustration $f$ is increased. 

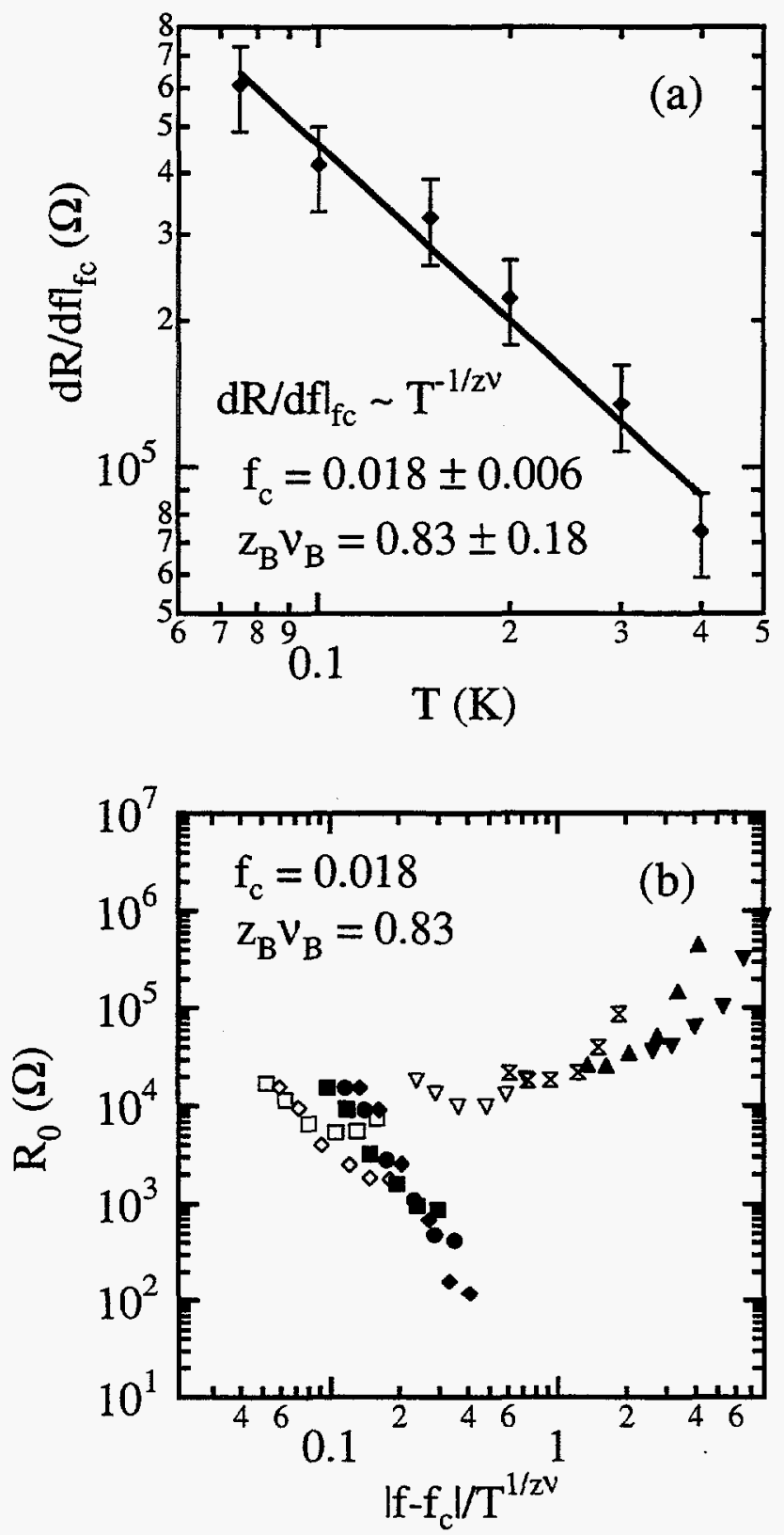

Figure 12.7: Results of the scaling analysis for the magnetic field-driven superconductorinsulator transition of the array for data from $T=75$ to $400 \mathrm{mK}$. In (a) is shown $\left(\mathrm{d} R_{0} / \mathrm{d} f\right) \mid f_{c}$ versus $T$ for the values of critical frustration and scaling exponents giving the straightest line, $f_{c}=0.018$ and $z_{B} \nu_{B}=0.83$. In (b) is shown the collapse of the data for $R_{0}$ of the array for the scaling function $\left|f-f_{c}\right| / T^{1 / z_{B} \nu_{B}}$. 


\section{Chapter 13}

\section{One-Dimensional Arrays of Josephson Junctions with Tunable Dissipation}

\subsection{Introduction}

Our experimental investigation of a two-dimensional (2D) array of Josephson junctions with tunable dissipation (see Chap. 11) prompted us to perform the same experiment with one-dimensional (1D) arrays. Having observed the current-voltage characteristics of the $2 \mathrm{D}$ array change from superconductor-like to insulator-like as the resistance of the ground plane was increased, and therefore the dissipation associated with the electrodynamic environment was decreased, we hoped a similar transition could be observed in $1 \mathrm{D}$ arrays. 1D arrays of normal metal junctions have been studied both experimentally and theoretically (for example, see [21,6]). On the other hand, 1D arrays of Josephson junctions have been studied theoretically (for example, see [10,9]), but have not been studied experimentally very extensively. ${ }^{1}$ To the best of our knowledge, no theoretical predictions exist for the effect of dissipation due to a ground plane on 1D Josephson junction arrays. Theoretical work investigating the effect of dissipation on ID arrays in the form of a shunt resistor across the junctions, and including quantum fluctuations due to the charging en-

\footnotetext{
${ }^{1}$ Capacitively coupled parallel 1D arrays and 2D arrays with length much greater than width (for example, see [91]) have been studied experimentally, but we have found little work on single row $1 D$ arrays (for a brief discussion of one such array, see [19]).
} 
ergy of the junctions, predict a superconductor-to-insulator transition as either the shunt resistance or charging energy is increased [9] (see also Chap. 4). In this chapter we describe our measurements of three $1 \mathrm{D}$ arrays over a ground plane with variable resistance. At low temperature, two of the arrays display insulator-like behavior, and one array displays superconductor-like behavior. By changing the resistance of the ground plane, we were able to change the current-voltage $(I-V)$ characteristics of the array. Unfortunately, we were unable to tune the superconducting array through a superconductor-like to insulator-like transition.

\subsection{Description of Samples}

Three different one-dimensional arrays over a tunable ground plane were fabricated and measured. Each of the three arrays is a chain of $441 \mathrm{Al}$ islands linked by 440 approximately $80 \times 80 \mathrm{~nm}^{2} \mathrm{Al} / \mathrm{Al}_{\mathbf{x}} \mathrm{O}_{\mathbf{y}} / \mathrm{Al}$ Josephson junctions for which we can continuously vary the dissipation associated with the local electrodynamic environment independently of any other relevant parameters. As in the case of the $2 \mathrm{D}$ arrays (see Chap. 11), we fabricated the arrays on $\mathrm{GaAs} / \mathrm{Al}_{0.3} \mathrm{Ga}_{0.7} \mathrm{As}$ heterostructures in which a two-dimensional electron gas (2DEG) is located approximately $100 \mathrm{~nm}$ from the surface. The heterostructures are described in detail in Chap. 6. The sheet density $n_{s}$, and thus the resistance per square $R_{g}$, of the $2 \mathrm{DEG}$ is changed by applying a large voltage $V_{B G}$ between the $2 \mathrm{DEG}$ and a metallic back gate.

\subsubsection{Description of Arrays}

As shown in Fig. 6.8, the junctions in the 1D arrays do not follow a straight line; instead, they following a meandering path. We expect the meandering path and straightline path arrays should behave in the same way, since the capacitance between an island and its neighbor to which it is not connected by a junction is very small compared to the junction capacitance and island capacitance to ground. All three 1D arrays have junctions with an area of approximately $80 \times 80 \mathrm{~nm}^{2}$ as measured from scanning electron micrographs of test arrays. These junction areas are slightly larger than those in the $2 \mathrm{D}$ array. We intentionally exposed the 1D arrays with a higher dose when performing electron-beam lithography to ensure there would be no broken links in the arrays. A 2D array with one broken link is usable; a $1 \mathrm{D}$ array with one broken link is an open circuit. Using the measured junction 


\begin{tabular}{|c|l|l|l|}
\hline Sample & $R_{N}(\mathrm{k} \Omega)$ & $C_{g}(\mathrm{fF})$ & $E_{J} / E_{C}$ \\
\hline \hline $\mathrm{A}$ & 2.75 & 1.7 & 6.2 \\
\hline $\mathrm{B}$ & 12.8 & 1.7 & 1.6 \\
\hline $\mathrm{C}$ & 17.6 & 2.9 & 1.8 \\
\hline
\end{tabular}

Table 13.1: Parameters of the three $1 D$ arrays.

area, and the specific capacitance for junctions of similar area and resistance determined by other groups [36] of $\approx 100 \mathrm{fF} / \mu \mathrm{m}^{2}$, we estimate a junction capacitance $C \approx 0.7 \mathrm{fF}$ for all three arrays. Other relevant parameters of the arrays are listed in Table 13.1. The three $1 \mathrm{D}$ arrays have normal state tunneling resistances $R_{N}=2.75,12.8$, and $17.6 \mathrm{k} \Omega$, measured from the inverse slope of the current-voltage $(I-V)$ characteristics of the arrays at high current bias when the arrays are driven normal by a $0.4 \mathrm{~T}$ magnetic field. The GaAs heterostructure substrate of sample $\mathrm{C}$ is substrate 2DEG-A as described in Chaps. 6 and 11. The substrates of samples $A$ and B, substrate 2DEG-B as described in Chap. 6 , contain a 2DEG with a sheet density $n_{s}$ smaller than that in sample $\mathrm{C}$, so that a lower back gate voltage $V_{B G}$ is needed to change the resistance per square of the ground plane $R_{g}$ by the same amount. The capacitance $C_{g}$ of each island to the $2 \mathrm{DEG}$ is measured as described in Chap. 9 from front gate voltage oscillations. The capacitance $C_{g}$ is $1.7 \pm 0.2 \mathrm{fF}$ in samples $\mathrm{A}$ and $\mathrm{B}$ and $2.9 \pm 0.2 \mathrm{fF}$ in sample $\mathrm{C}$. For all three samples, $C_{g}$ is measured for different back gate voltages $V_{B G}$ and found to be independent of $V_{B G}$.

We estimate the Josephson coupling energy $E_{J}$ of the arrays through the relation $E_{J}=(\Delta / 2) /\left(R_{Q} / R_{N}\right)$, where $\Delta$ is the superconducting energy gap (see Chap. 11) measured from the $I-V$ characteristics of the array at large current and voltage. The values of $2 \Delta$ for samples $\mathrm{A}, \mathrm{B}$, and $\mathrm{C}$ are $0.31,0.36$, and $0.38 \mathrm{meV}$, respectively. Combining these values of $2 \Delta$ with the values of $R_{N}$ gives $E_{J} / k_{B}=2.1,0.53$, and $0.41 \mathrm{~K}$ for samples $\mathrm{A}, \mathrm{B}$, and $\mathrm{C}$, respectively.

The charging energy of a single isolated junction is $E_{C}=e^{2} / 2 C$. Following the convention used in defining the charging energy for a single electron transistor [5], we define the charging energy for a $1 \mathrm{D}$ array as $E_{C_{\Sigma}} \equiv e^{2} / 2 C_{\Sigma}$ where $C_{\Sigma}$ is the effective capacitance seen by an island in an infinite $1 \mathrm{D}$ array $C_{\Sigma}=\left(C_{g}^{2}+4 C C_{g}\right)^{1 / 2} \cdot{ }^{2}$ Using the estimated value

\footnotetext{
${ }^{2}$ For a single electron transistor, a single island with two tunnel junctions with capacitances $C_{1}$ and $C_{2}$ and capacitance to ground $C_{g}$, the charging energy is defined as $e^{2} / 2 C_{\Sigma}$, where $C_{\Sigma}$ is the sum of the
} 
of $C$ and measured values of $C_{g}$, we calculate the values of the charging energy $E_{C_{\Sigma}} / k_{B}$ (from now on in this chapter $E_{C_{\Sigma}}$ will be referred to as $E_{C}$ ) to be $0.34 \mathrm{~K}$ for samples $\mathrm{A}$ and $\mathrm{B}$, and $0.23 \mathrm{~K}$ for sample $\mathrm{C}$. Using the determined values of $E_{C}$ and $E_{J}$, the ratio of Josephson energy to charging energy for samples $\mathrm{A}, \mathrm{B}$ and $\mathrm{C}$ is given by $E_{J} / E_{C}=6.2,1.6$ and 1.8, respectively. We note that samples $B$ and $C$ have approximately the same value of $E_{J} / E_{C}$.

\subsubsection{Description of Ground Planes}

The resistance per square of the ground plane $R_{g}$ was measured using a van der Pauw technique, and the sheet density $n_{s}$ was obtained from Shubnikov-de Haas oscillations at magnetic fields of 0.1 to $0.4 \mathrm{~T}$. At a back gate voltage $V_{B G}=0$, the $\mathrm{GaAs} / \mathrm{Al}_{0.3} \mathrm{Ga}_{0.7} \mathrm{As}$ heterostructure used as the substrate in sample $C$ has a sheet density $n_{s} \approx 2.05 \times 10^{11} \mathrm{~cm}^{-2}$ and resistance per square of the ground plane $R_{g} \approx 110 \Omega / \mathrm{sq}$. For $V_{B G}=0$, the heterostructure substrates of samples $A$ and $B$ have sheet densities $n_{s} \approx 1.5 \times 10^{11} \mathrm{~cm}^{-2}$ and ground plane resistance $R_{g} \approx 110-120 \Omega / \mathrm{sq}$. For both heterostructures, $R_{g}$ could be increased from approximately $110-120 \Omega / \mathrm{sq}$ for $V_{B G}=0$ to greater than $1 \mathrm{G} \Omega$ at higher $V_{B G}$. The resistance of the $2 \mathrm{DEG}$ reaches values greater than $1 \mathrm{G} \Omega$ at $V_{B G} \approx 450 \mathrm{~V}$ in samples $\mathrm{A}$ and $\mathrm{B}$ and at $V_{B G} \approx 650 \mathrm{~V}$ in sample $\mathrm{C}$. Below $R_{g} \approx 10 \mathrm{k} \Omega, R_{g}$ is measured to be temperature independent; at the higher back gate voltages corresponding to $R_{g} \geq 500 \mathrm{k} \Omega$, $R_{g}$ changed by as much as a factor of 2 when the temperature was increased from 25 to 300 $\mathrm{mK}$. As we increase $V_{B G}$ to high voltages, the 2DEG undergoes a phase transition from metallic to insulating that may complicate the analysis of the array data at high $V_{B G}$ and $R_{g}$.

\subsection{Current-Voltage Characteristics}

This section discusses the general properties of the current-voltage $(I-V)$ characteristics of the three $1 D$ arrays. Sample A displays superconductor-like behavior, and samples $\mathrm{B}$ and $\mathrm{C}$ show insulator-like behavior at low temperatures. The effect of changing the ground plane resistance on the general properties of the $I-V$ curves, such as the critical

capacitances seen by the island $C_{1}+C_{2}+C_{g}$. In an infinite $1 \mathrm{D}$ array with junction capacitances $C$ and capacitances to ground $C_{g}$ of the islands, the effective capacitance seen by an island is $C_{\Sigma}=C_{g}+2 C_{h}$, where $C_{h}$ is the capacitance seen from the edge of a half-infinite array, $C_{h}^{-1}=C^{-1}+\left(C_{g}+C_{h}\right)^{-1}$, so that $C_{\Sigma}=\left(C_{g}^{2}+4 C C_{g}\right)^{1 / 2}$. 
current in the superconducting array and the threshold voltage in the insulating arrays, is discussed.

\subsubsection{General Properties}

Shown in Fig. 13.1 are the $I-V$ characteristics of all three $1 \mathrm{D}$ arrays at $T=$ $20 \mathrm{mK}$, zero magnetic field, and $V_{B G}=0$ (corresponding to $R_{g} \approx 110-120 \Omega / \mathrm{sq}$ ). The $I-V$ characteristic for sample $\mathrm{A}\left(R_{N}=2.75 \mathrm{k} \Omega\right)$ is clearly superconductor-like, with a supercurrent feature over the range of array current of approximately $-18 \mathrm{nA}$ to $18 \mathrm{nA}$. The curve is hysteretic, with the direction of the current sweep indicated by the arrows. The $I-V$ characteristics of samples $\mathrm{B}\left(R_{N}=12.8 \mathrm{k} \Omega\right)$ and $\mathrm{C}\left(R_{N}=17.6 \mathrm{k} \Omega\right)$ are both insulatorlike, with a Coulomb gap near zero voltage. The gap feature in both $I-V$ characteristics is approximately $4 \mathrm{mV}$ wide. At array voltages just above the gap, the array current rises above zero and a supercurrent-like feature develops that extends for approximately 100-150 pA. The array with the slightly higher ratio of $E_{J} / E_{C}$ has both a slightly larger Coulomb gap and supercurrent-like feature. In summary, the $I-V$ characteristic of sample A shows superconductor-like behavior, and those of sample $B$ and $C$ show insulator-like behavior. Since the $1 \mathrm{D}$ arrays with $E_{J} / E_{C} \leq 2$ show insulating behavior, and that with $E_{J} / E_{C} \geq 6$ shows superconducting behavior, we predict there will be a superconductorinsulator transition as a function of $E_{J} / E_{C}$ in the range $2<E_{J} / E_{C}<6$.

\subsubsection{Effect of Dissipation on Supercurrent}

As the ground plane resistance $R_{g}$ in the array displaying superconductor-like behavior, sample $\mathrm{A}$, is increased, the $I-V$ characteristic of the array changes. Shown in Fig. 13.2 are the $I-V$ characteristics of sample A for four different values of $R_{g}$ ranging from $110 \Omega / \mathrm{sq}$ to $>1 \mathrm{G} \Omega / \mathrm{sq}$. The size of the supercurrent increases as $R_{g}$ is increased, reaching a maximum value at approximately $R_{g}=12 \mathrm{k} \Omega / \mathrm{sq}$ and then decreasing again. At approximately $R_{g}=12 \mathrm{k} \Omega / \mathrm{sq}$, the $I-V$ characteristic also becomes non-hysteretic.

The change in the size of the supercurrent with increasing $R_{g}$ is quantified in Fig. 13.3 showing the critical current of the array $I_{c}$ versus $\log R_{g}$. The critical current $I_{c}$ is defined as the array current for which the array voltage rises above approximately twice the noise level of $10 \mu \mathrm{V}$. $I_{c}$ increases with a nearly exponential dependence on $R_{g}$ until it reaches a maximum at $R_{g} \approx 12 \mathrm{k} \Omega / \mathrm{sq}$. At $R_{g}>12 \mathrm{k} \Omega / \mathrm{sq}, I_{c}$ decreases with increasing $R_{g}$. 

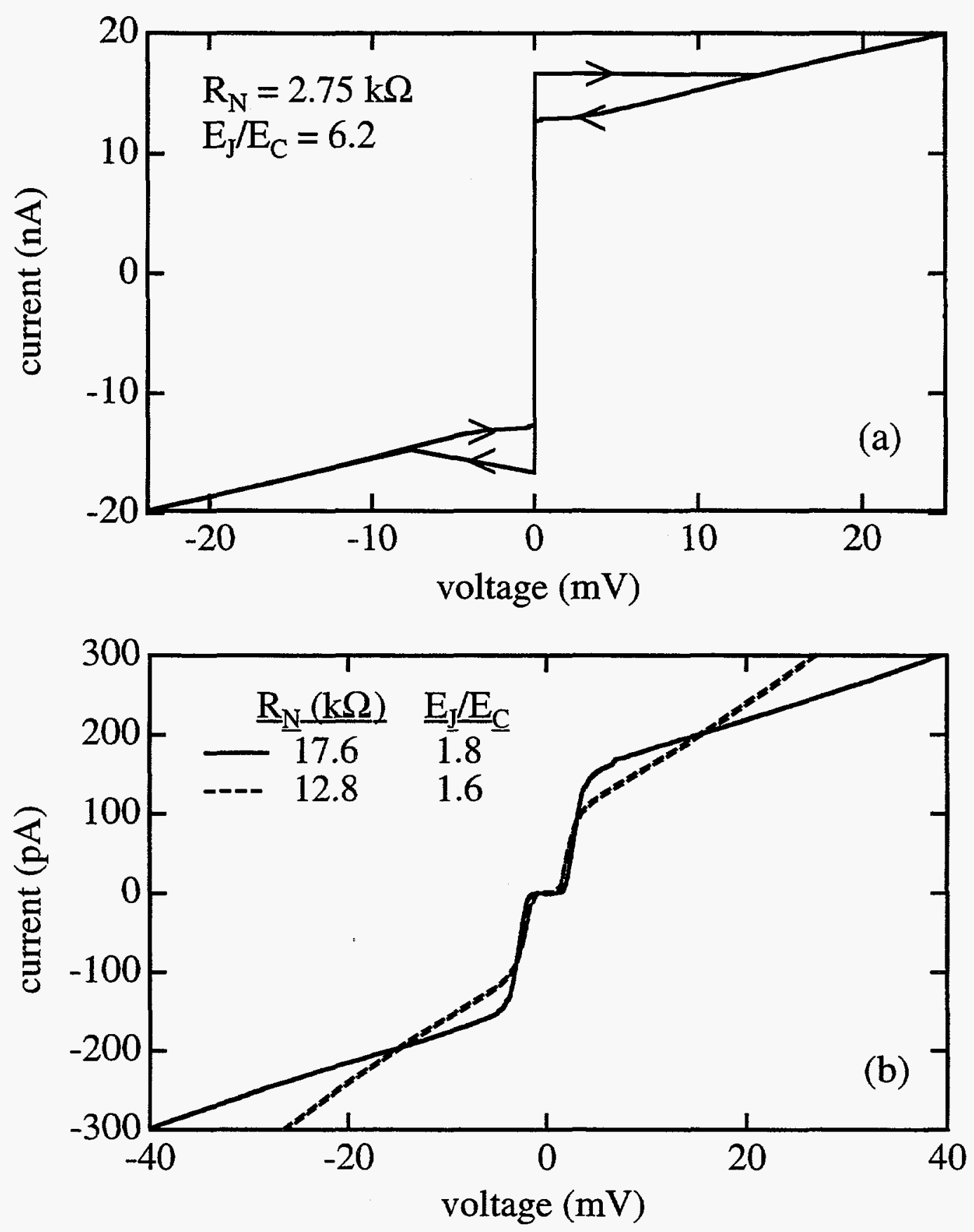

Figure 13.1: $I-V$ characteristics of the $1 \mathrm{D}$ arrays at $T=20 \mathrm{mK}$, zero magnetic field, and $V_{B G}=0\left(R_{g}=110-120 \Omega / \mathrm{sq}\right)$. Shown in (a) is the $I-V$ characteristic for sample A $\left(R_{N}=2.75 \mathrm{k} \Omega\right.$ ), with the arrows indicating the direction of the sweep. Shown in (b) are the $I-V$ characteristics for samples B $\left(R_{N}=12.8 \mathrm{k} \Omega\right)$ and $\mathrm{C}\left(R_{N}=17.6 \mathrm{k} \Omega\right)$. 


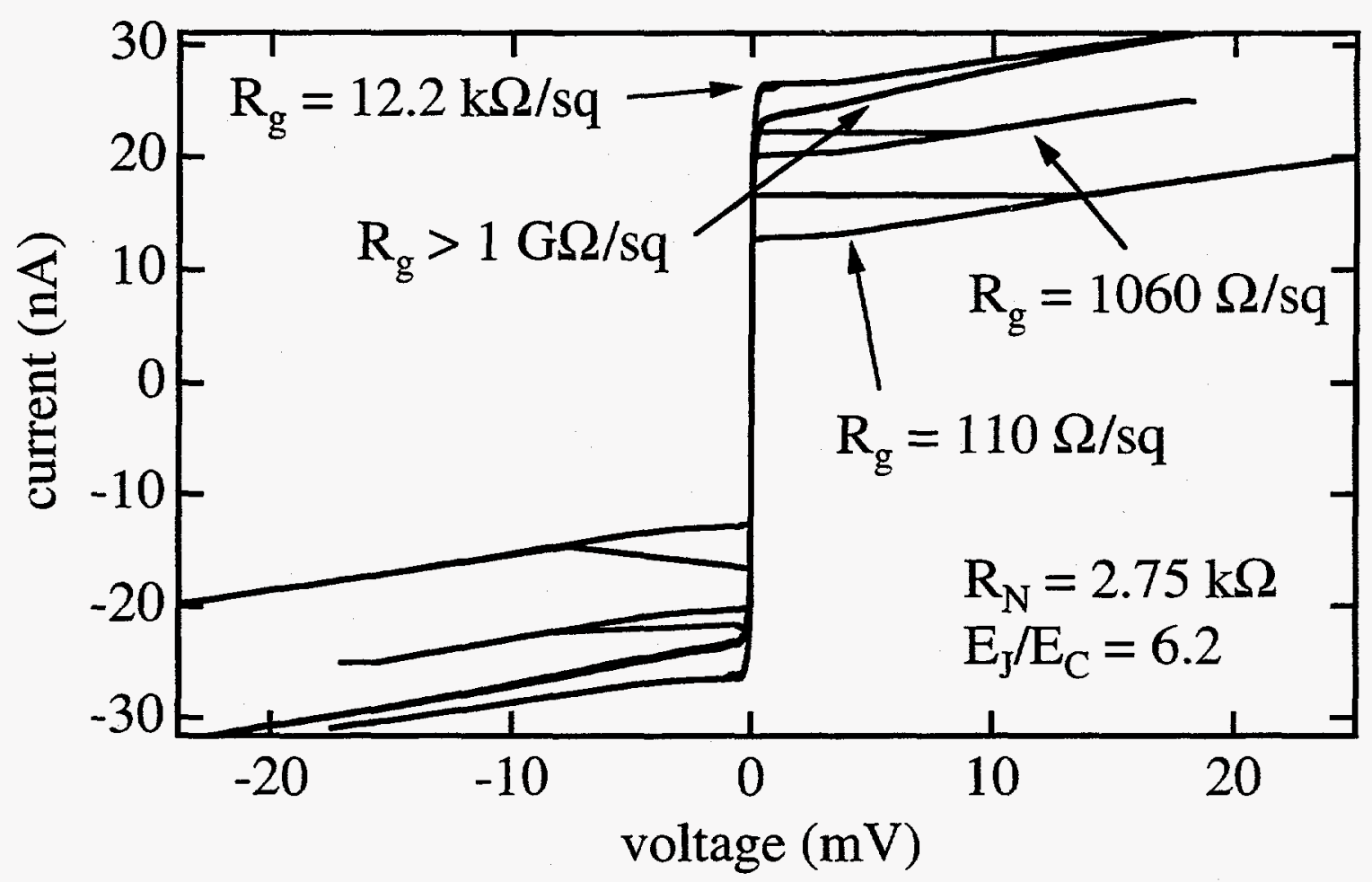

Figure 13.2: $I-V$ characteristics of sample $\mathrm{A}\left(R_{N}=2.75 \mathrm{k} \Omega\right)$ at $T=20 \mathrm{mK}$ and zero magnetic field for four different ground plane resistances $R_{g}=110,1060,12.2 \mathrm{k}$, and $>1 \mathrm{G} \Omega / \mathrm{sq}$, corresponding to $V_{B G}=0,300,400$, and $450 \mathrm{~V}$. The size of the supercurrent increases with increasing $R_{g}$, reaching a maximum at $R_{g} \approx 12 \mathrm{k} \Omega / \mathrm{sq}$. The size of the supercurrent then decreases with increasing $R_{g}$, and the $I-V$ characteristics become nonhysteretic. 


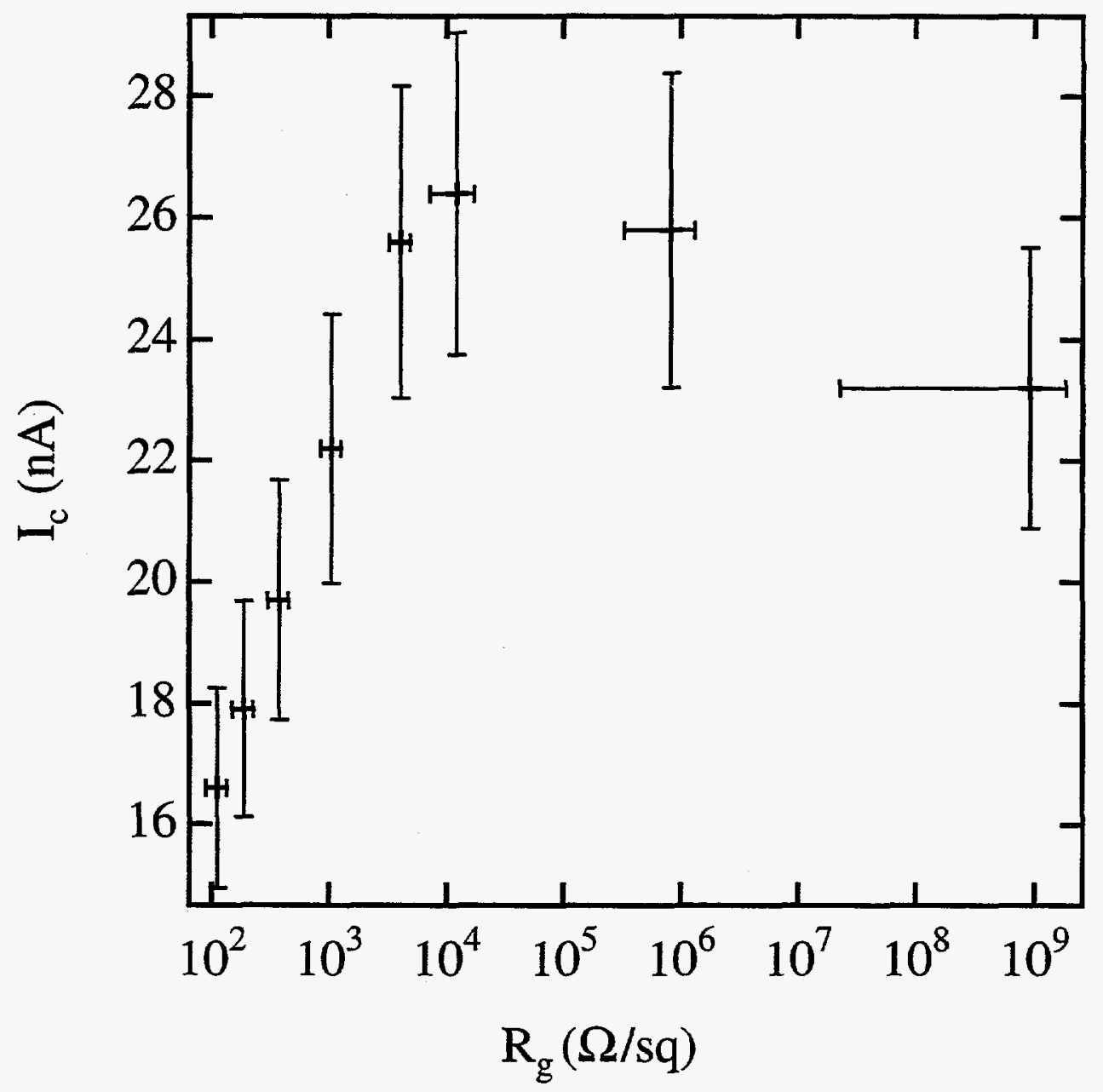

Figure 13.3: Critical current $I_{c}$ of the array versus ground plane resistance $R_{g}$ for sample $A$ at $\mathrm{T}=20 \mathrm{mK}$. 


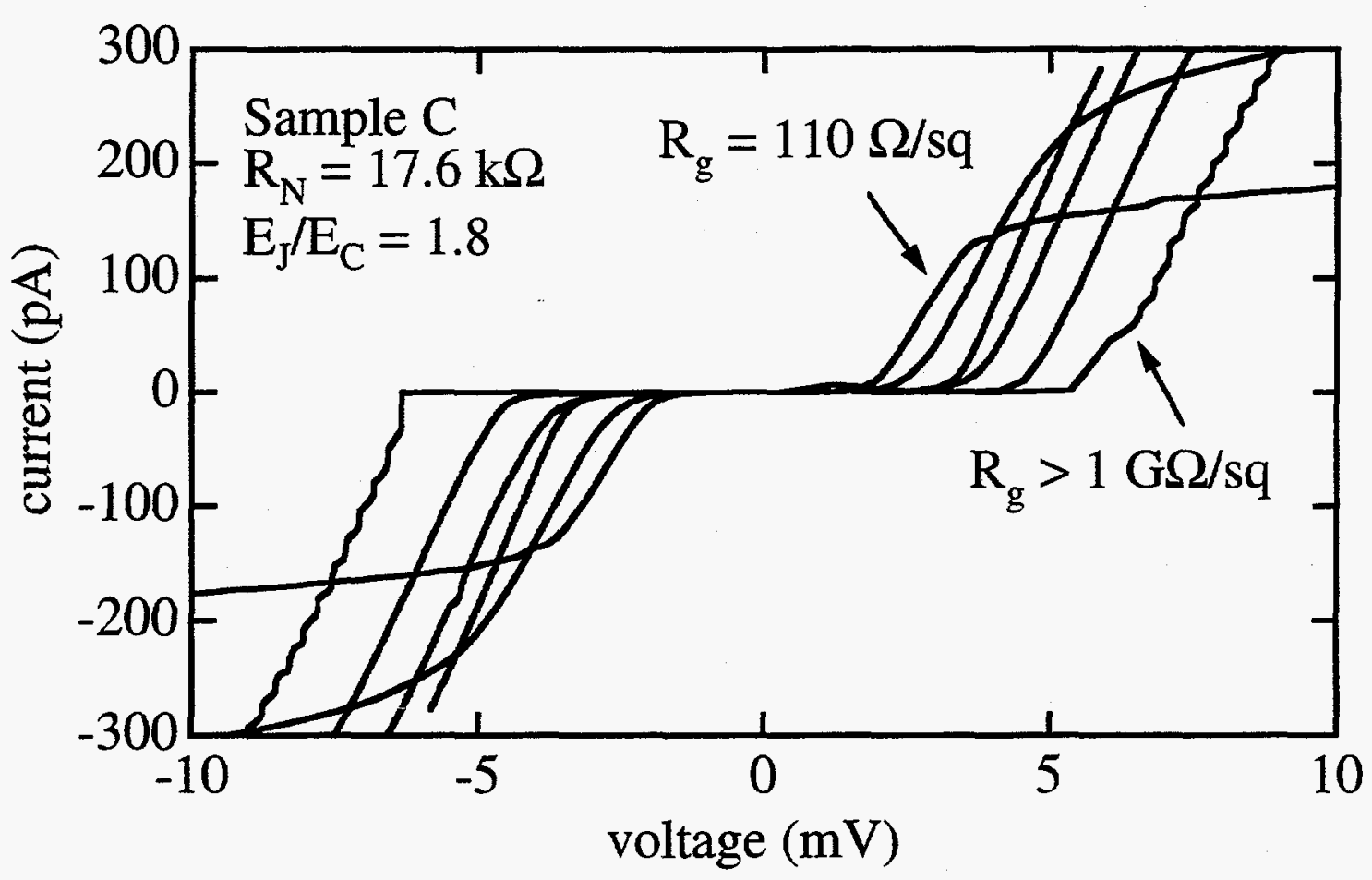

Figure 13.4: $I-V$ characteristics of sample $\mathrm{C}\left(R_{N}=17.6 \mathrm{k} \Omega\right)$ at $T=20 \mathrm{mK}$ and zero magnetic field for six different ground plane resistances $R_{g}=110,1220,9760,32.1 \mathrm{k}, 1.22$ $\mathrm{M}$, and $>1 \mathrm{G} \Omega / \mathrm{sq}$, corresponding to $V_{B G}=0,450,600,625,650$, and $700 \mathrm{~V}$. The size of the Coulomb gap increases by more than a factor of 4 as the ground plane resistance $R_{g}$ is increased from 110 to $>1 \mathrm{G} \Omega / \mathrm{sq}$.

We currently have no explanation for the increase of $I_{c}$ with $R_{g}$ for $R_{g} \leq 12 \mathrm{k} \Omega / \mathrm{sq}$. We see similar behavior in the supercurrent-like feature above the Coulomb gap in the insulating arrays, samples B and C.

\subsubsection{Effect of Dissipation on Coulomb Gap}

As the ground plane resistance $R_{g}$ in the insulating arrays, samples $\mathrm{B}$ and $\mathrm{C}$, is increased, the $I-V$ characteristics of the arrays change. Shown in Fig. 13.4 are the $I-V$ characteristics for sample $\mathrm{C}$ for six different values of ground plane resistance $R_{g}$ ranging from $110 \Omega / \mathrm{sq}$ to $>1 \mathrm{G} \Omega / \mathrm{sq}$. The change in the $I-V$ characteristics as a function of $R_{g}$ for sample B is similar to that in sample C. The size of the Coulomb gap increases with increasing $R_{g}$. The size of the supercurrent-like feature right outside the Coulomb gap also increases with increasing $R_{g}$. 
The increase in the size of the Coulomb gap with increasing $R_{g}$ is quantified in Fig. 13.5 showing the threshold voltage $V_{T}$ of the insulating arrays, samples $\mathrm{B}$ and $\mathrm{C}$, versus the log of the ground plane resistance $R_{g}$. The threshold voltage $V_{T}$ is defined as the voltage for which the current rises above twice the noise level of approximately 0.1 pA. Fig. 13.5 (a) shows the threshold voltage $V_{T}$ versus $\log R_{g}$ for sample $B$. The upper curve shows the data for the array with the islands in the superconducting state (zero applied magnetic field), and the lower curve shows the data for the array with the islands in the normal state (0.4 T applied magnetic field). Fig. 13.5 (b) shows the same information for sample C. In general, $V_{T}$ increases with increasing $R_{g}$ for the arrays with the islands in both the superconducting and normal state. We expect this increase in $V_{T}$ with increasing $R_{g}$, since as $R_{g}$ is increased, dissipation is decreased, damping of phase fluctuations is decreased, and therefore the array properties should become more insulating, and the size of the Coulomb gap should increase.

\subsection{Zero-Bias Resistance of Arrays}

We measure the zero-bias resistance $R_{0}$ of the arrays as a function of temperature $T$ and ground plane resistance $R_{g}$. Above $R_{0} \approx 1 \mathrm{k} \Omega, R_{0}$ is determined from the inverse slope of the $I-V$ characteristics near zero current and voltage (typically the voltage range $\pm 100 \mu \mathrm{V}$ is used). Below $R_{0} \approx 1 \mathrm{k} \Omega, R_{0}$ is measured using a lock-in technique. Fig. 13.6 shows the zero-bias resistance $R_{0}$ for all three arrays in the superconducting state (zero applied magnetic field) versus temperature for different values of the ground plane resistance $R_{g}$. Sample A shows superconductor-like behavior, with $R_{0}$ decreasing steadily with decreasing $\mathrm{T}$ for all the values of $R_{g}$. At the lowest temperatures, $R_{0}$ appears to be reaching a constant value, and does not rapidly drop towards zero as would be expected in a superconductor. We are unsure if this flattening of $R_{0}$ is real or due to sample heating or to the limit of our measurement techniques. For all temperatures shown here, $R_{0}$ for sample $\mathrm{A}$ increases with increasing $R_{g}$, with the increase being greatest at the lowest temperatures. At $\mathrm{T}=20 \mathrm{mK}, R_{0}$ increases by nearly 2 orders of magnitude as $R_{g}$ is changed from $110 \Omega / \mathrm{sq}$ to $>1 \mathrm{G} \Omega / \mathrm{sq}$. Unfortunately, we were not able to tune this sample through a superconductor-like to insulator-like transition, despite being able to change the ground plane resistance over 7 orders of magnitude.

Samples $\mathrm{B}$ and $\mathrm{C}$ show insulating behavior, with $R_{0}$ increasing steadily with de- 

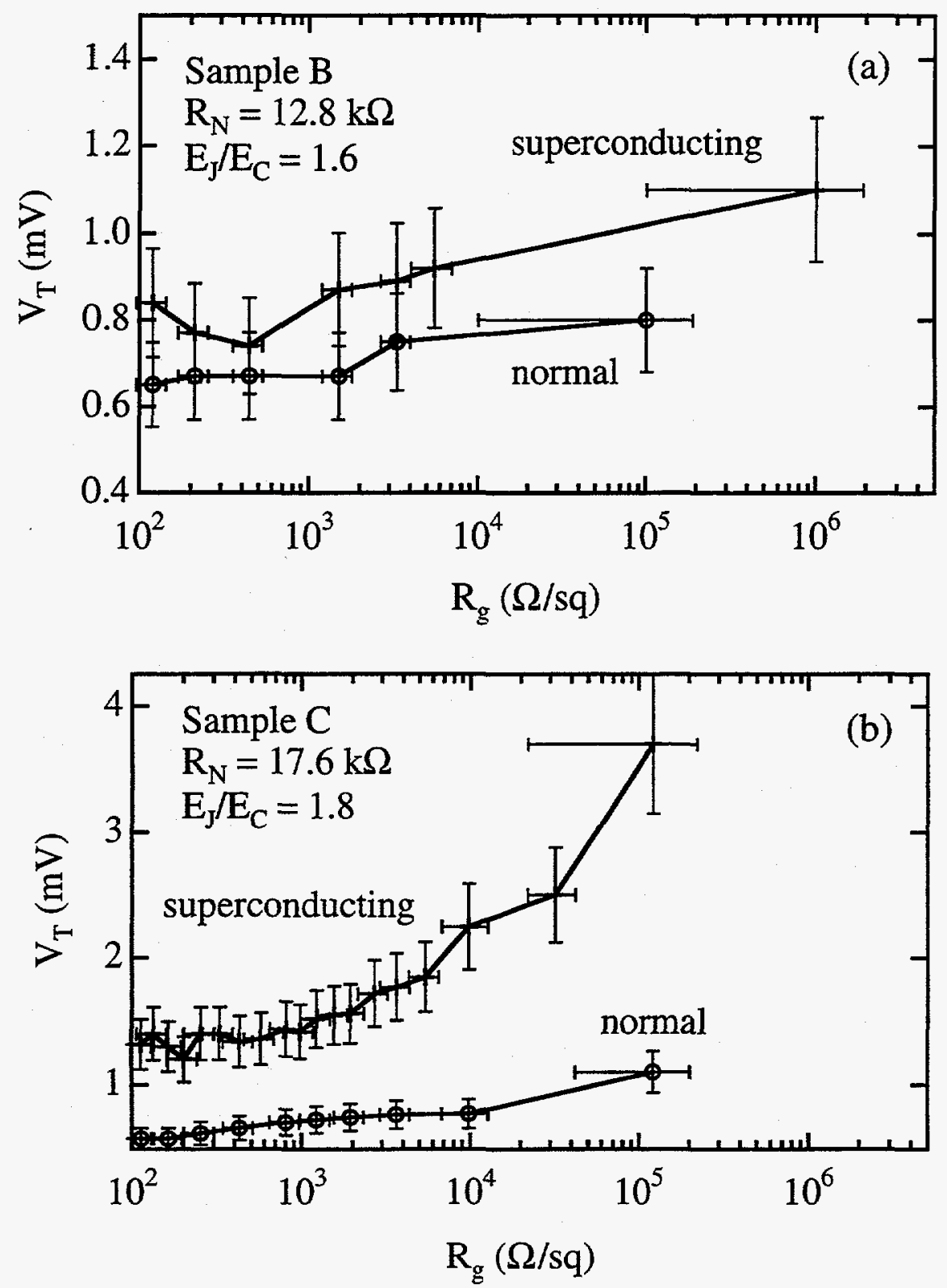

Figure 13.5: Threshold voltage $V_{T}$ of the array versus ground plane resistance $R_{g}$ for samples (a) B and (b) C. Data for the islands of the arrays in both the superconducting state and normal state are shown. 

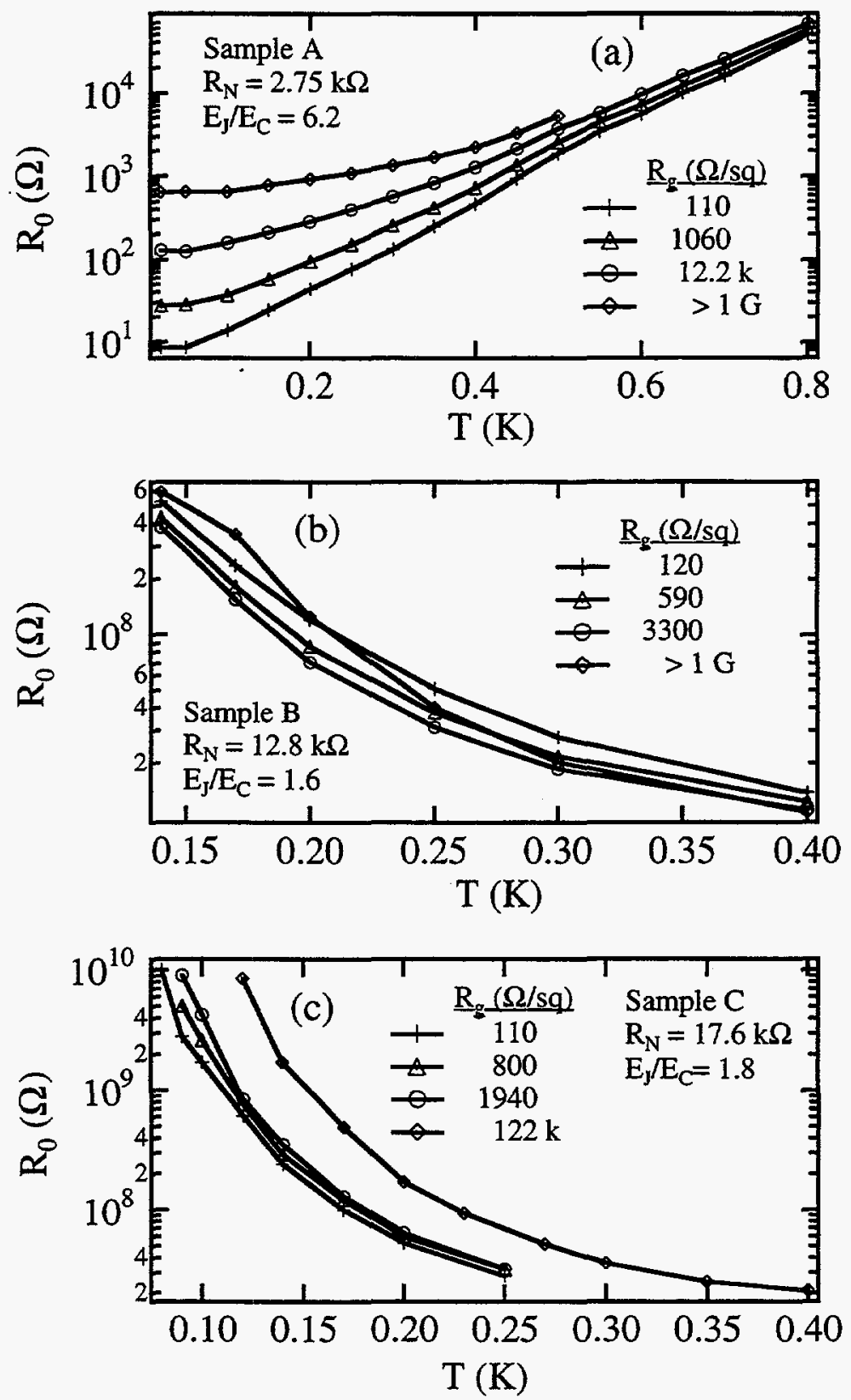

Figure 13.6: Zero-bias resistance of the array $R_{0}$ versus temperature $T$ for samples (a) A, (b) $\mathrm{B}$, and (c) $\mathrm{C}$ at zero applied magnetic field for four different values of the ground plane resistance $R_{g}$. 
creasing $\mathrm{T}$ for all values of $R_{g}$. In sample $\mathrm{C}, R_{0}$ increases with increasing $R_{g}$ for all the temperatures shown here. In sample $\mathrm{B}, R_{0}$ increases with increasing $R_{g}$ at the lower temperatures (below $\approx 200 \mathrm{mK}$ ), but at higher temperatures, $R_{0}$ decreases slightly with increasing $R_{0}$. In general, we expect that $R_{0}$ should increase with increasing $R_{g}$, since increasing $R_{g}$ decreases dissipation and reduces damping of the phase fluctuations. Shown in Fig. 13.7 for comparison of the superconducting sample and one insulating sample is $R_{0}$ versus temperatures for samples $\mathrm{A}$ and $\mathrm{C}$ plotted on the same graph. By examining this data, we predict we will be able to tune a $1 \mathrm{D}$ array with a ratio of $E_{J} / E_{C} \approx 4$ through a superconductorto-insulator transition.

In Fig. 13.8 is shown the zero-bias resistance $R_{0}$ for samples $\mathrm{A}$ and $\mathrm{B}$ with the islands of the array in the normal state (0.4 $\mathrm{T}$ applied magnetic field) versus temperature for different values of the ground plane resistance $R_{g}$. Samples $\mathrm{A}$ and $\mathrm{B}$ show insulating behavior with $R_{0}$ increasing with increasing $R_{g}{ }^{3}$ This behavior is expected, since the islands are in the normal state, the Josephson energy $E_{J}=0$, and only charging effects are important. The array with the larger value of normal state tunneling resistance $R_{N}$, sample $\mathrm{B}$, has a larger value of $R_{0}$ than the lower $R_{N}$ array, sample $\mathrm{A}$, for the entire temperature range shown, with the difference being greatest at the lower temperatures. For both samples $\mathrm{A}$ and $\mathrm{B}$, the zero-bias resistance of the array $R_{0}$ increases with increasing $R_{g}$ for all temperatures shown. For samples $\mathrm{B}$ and $\mathrm{C}$ (not shown), the values of $R_{0}$ as a function of temperature are similar to those with the islands of the array in the superconducting state. In sample $\mathrm{A}, R_{0}$ is about 6 orders of magnitude larger at $20 \mathrm{mK}$ with the islands in the normal state than with the islands in the superconducting state. For samples $\mathrm{A}$ and $\mathrm{B}$, the increase in $R_{0}$ as a function of $R_{g}$ with the islands in the normal state is not as great as with the islands in the superconducting state; the greatest change in $R_{0}$ is seen in sample A at $20 \mathrm{mK}$, where $R_{0}$ changes by $25 \%$ as $R_{g}$ is increased from $110 \Omega / \mathrm{sq}$ to $>1 \mathrm{G} \Omega / \mathrm{sq}$.

In our experiment with a $2 \mathrm{D}$ array with variable dissipation (see Chap. 11), we find that $R_{0}$ increases exponentially with $R_{g}$, with the dependence being strongest at the lowest temperatures. We plot $R_{0}$ versus $R_{g}$ in a variety of ways for the $1 \mathrm{D}$ arrays to determine if there is a similar exponential dependence. We find that $R_{0}$ seems to increase faster than exponentially with $R_{g}$. Shown in Fig. 13.9 for sample $\mathrm{A}$ is $R_{0}$ versus $R_{g}$ on a $\log -\log$ plot for temperatures ranging from 20 to $800 \mathrm{mK}$.

\footnotetext{
${ }^{3}$ For sample $\mathrm{C}$, we have $R_{0}$ versus temperature data for only one value of $R_{g}$ (the data is not shown here), but we expect $R_{0}$ will also increase with $R_{g}$.
} 


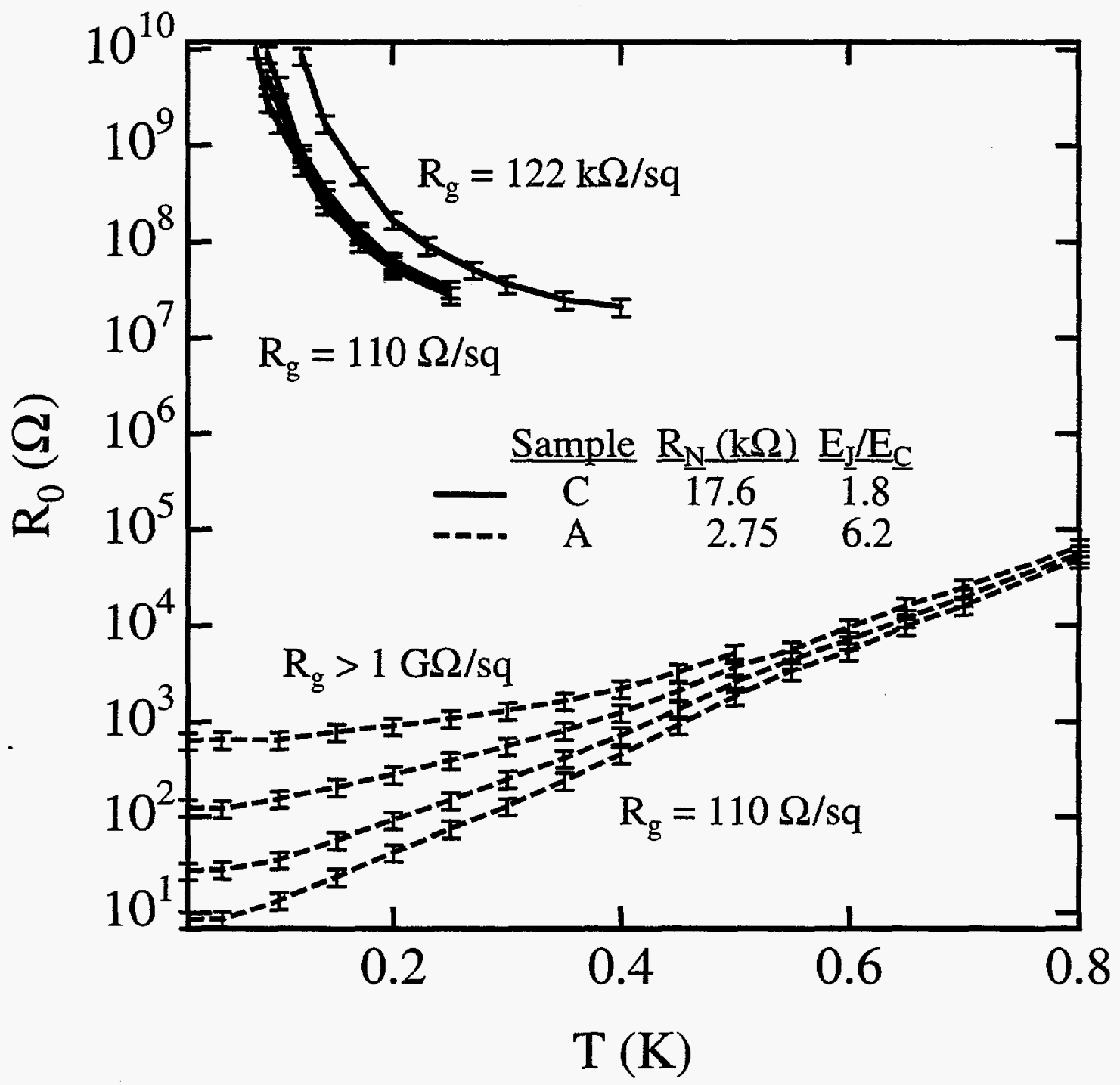

Figure 13.7: Zero-bias resistance of the array $R_{0}$ versus temperature $T$ for samples $A$ and $\mathrm{C}$ at zero applied magnetic field for four different values of the ground plane resistance $R_{g}$. This is the same data as shown in Fig. 13.6 for samples $A$ and $C$, but with the data from the two samples plotted on the same graph for comparison. The values of the ground plane resistance are $R_{g}=110,1060,12.2 \mathrm{k}$, and $>1 \mathrm{G} \Omega / \mathrm{sq}$ for sample $\mathrm{A}$ and $110,800,1940$, and $122 \mathrm{k} \Omega / \mathrm{sq}$ for sample $\mathrm{B}$, from bottom to top. 

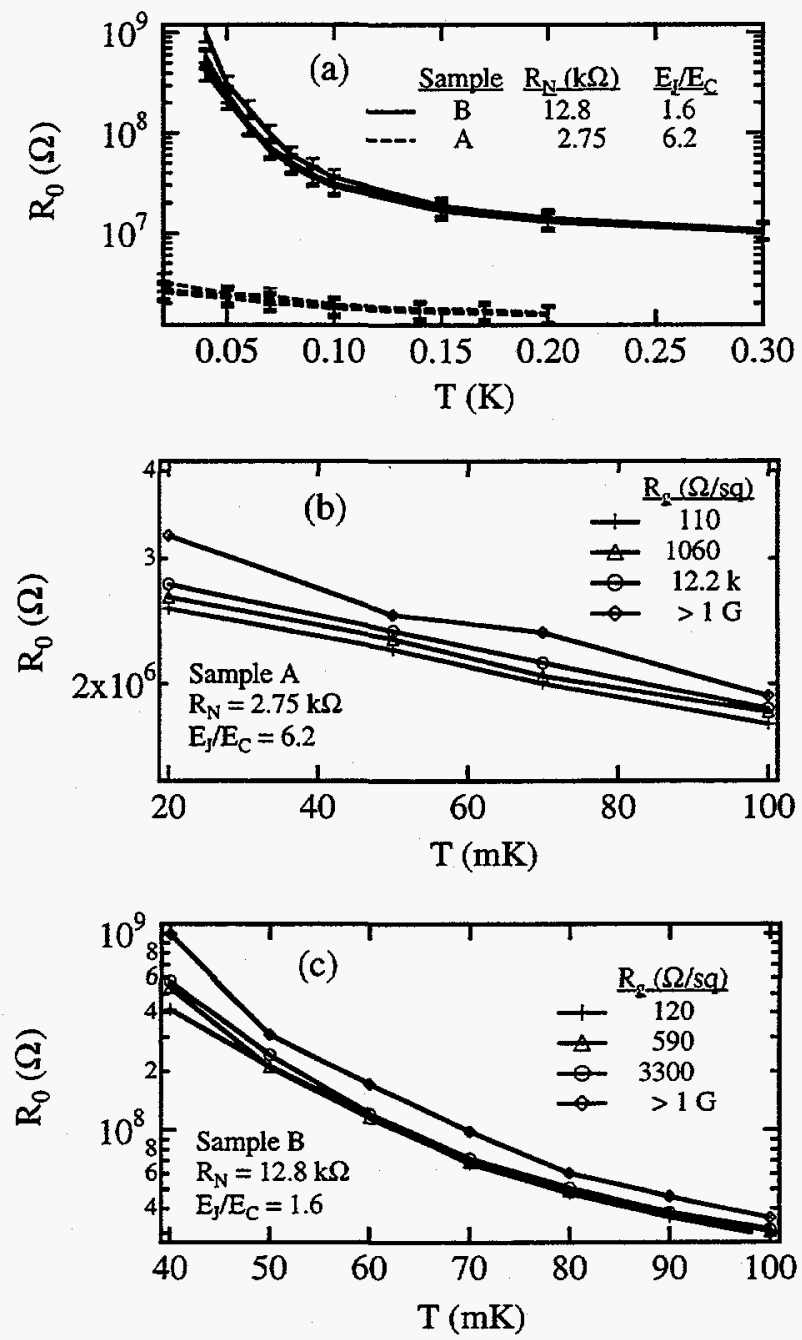

Figure 13.8: In (a) is shown zero-bias resistance $R_{0}$ of the array versus temperature $T$ for samples $\mathrm{A}$ and $\mathrm{B}$ for $0.4 \mathrm{~T}$ applied magnetic field (islands in the normal state) for different values of the ground plane resistance $R_{g}$. In (b) is shown $R_{0}$ versus $T$ for sample A (from (a)) for the temperature range $T=20$ to $100 \mathrm{mK}$ for $R_{g}=110,1060,12.2 \mathrm{k}$, and $>1 \mathrm{G}$ $\Omega /$ sq. In (c) is shown $R_{0}$ versus $T$ for sample B (from (a)) for the temperature range $T=$ 40 to $100 \mathrm{mK}$ for $R_{g}=120,590,3300$, and $>1 \mathrm{G} \Omega / \mathrm{sq}$. 


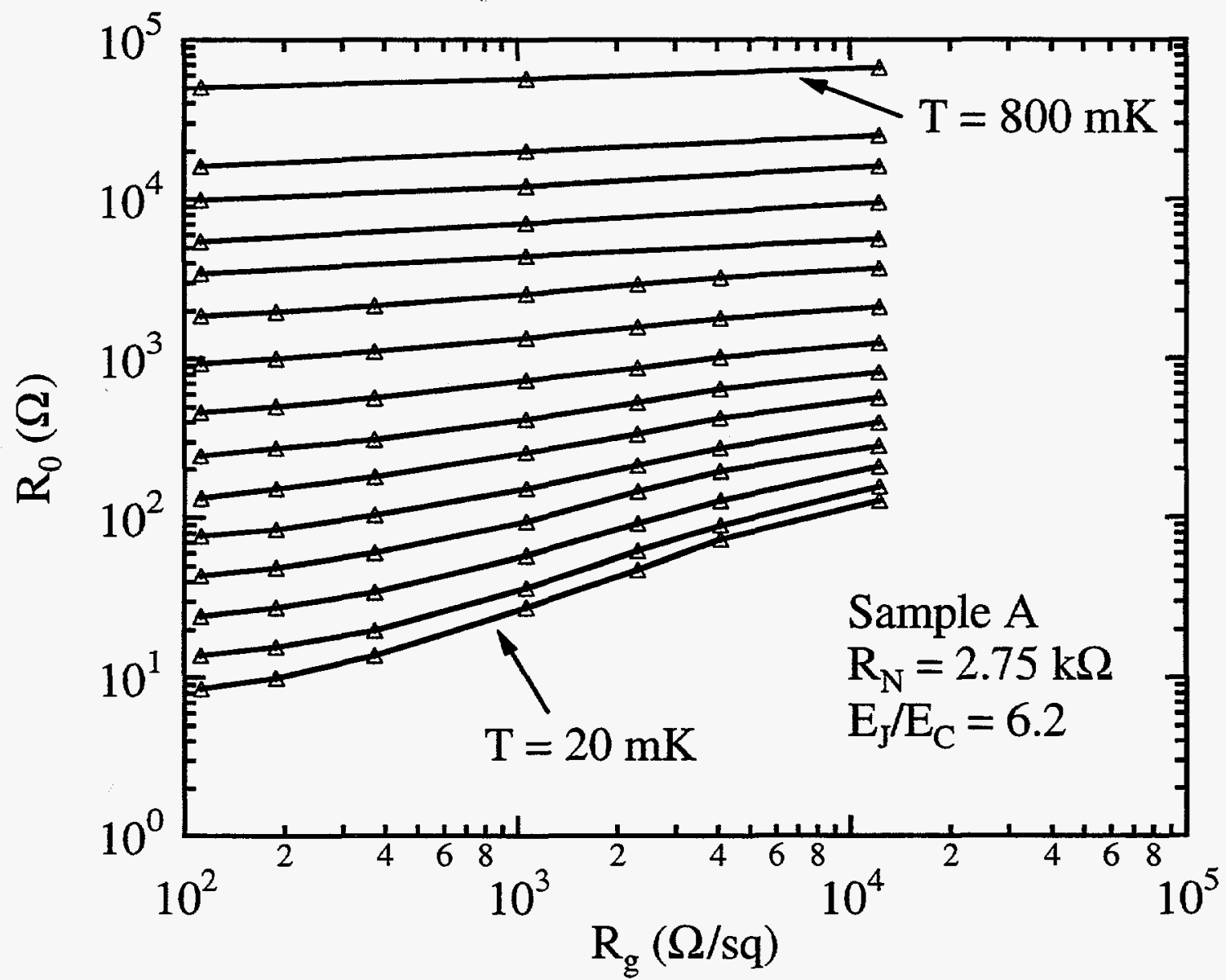

Figure 13.9: Zero-bias resistance of the array $R_{0}$ versus ground plane resistance $R_{g}$ for sample $A$ at zero applied magnetic field for fifteen different values of temperature $T=20$, $100,150,200,250,300,350,400,450,500,550,600,650,700$, and $800 \mathrm{mK}$, from bottom to top. 


\begin{tabular}{|c|l|l|l|}
\hline Sample & $E_{a S} / k_{B}(K)$ & $E_{a N} / k_{B}(K)$ & $E_{C} / k_{B}(K)$ \\
\hline \hline $\mathrm{A}$ & - & 0.027 & 0.34 \\
\hline $\mathrm{B}$ & 0.80 & 0.18 & 0.34 \\
\hline $\mathrm{C}$ & 0.68 & 0.18 & 0.23 \\
\hline
\end{tabular}

Table 13.2: Activation energies for samples A, B, and $C$ for zero magnetic field (superconducting islands) $\left(E_{a S}\right)$ and $0.4 \mathrm{~T}$ magnetic field (normal islands) $\left(E_{a N}\right) . E_{a}$ is determined by fitting a line to plots of $\ln R_{0}$ versus $1 / \mathrm{T}$. For comparison, the charging energies $E_{C}\left(E_{C_{\Sigma}}\right)$ for the three samples are also listed.

\subsection{Activation Energy}

If the current in a system arises from thermally activated behavior, the resistance will follow the Arrhenius form:

$$
R=R_{1} \exp \left(E_{a} / k_{B} T\right)
$$

where $E_{a}$ is the activation energy and $R_{1}$ is a constant.

To determine if our $1 \mathrm{D}$ arrays show thermally activated behavior, we plot $\ln R_{0}$ versus $1 / T$. Shown in Fig 13.10 is $\ln R_{0}$ versus $1 / T$ for sample $C$ with the islands of the array in the superconducting state (zero magnetic field) for four different values of $R_{g}$ ranging from $110 \Omega / \mathrm{sq}$ to $1.22 \mathrm{M} \Omega / \mathrm{sq}$. The symbols are the data points, and the lines are linear fits to the data. We see that sample $\mathrm{C}$ displays thermally activated behavior in the temperature range 90 to $450 \mathrm{mK}$, and $E_{a}$ increases with increasing $R_{g}$. Thermally activated behavior is seen over the temperature range $T \approx 40$ to $300 \mathrm{mK}$ with the islands in the superconducting state for samples $\mathrm{B}$ and $\mathrm{C}$, and with the islands in the normal state for all three samples. The values of $E_{a}$ for samples $\mathrm{A}, \mathrm{B}$, and $\mathrm{C}$ for the smallest value of $R_{g}$ are given in Table 13.2 . In general, $E_{a}$ increases with increasing $R_{g}$. Sample $A$ did not display thermally activated behavior with the islands in the superconducting state (zero applied magnetic field).

As observed experimentally, the arrays are expected to show thermally activated behavior over only a intermediate temperature range [19]. At low temperatures, temperature-independent quantum fluctuations are thought to dominate the resistance. At high temperatures, the superconducting energy gap becomes temperature dependent. There are theoretical predictions for the activation energy $E_{a}$ of $1 \mathrm{D}$ and $2 \mathrm{D}$ arrays in the normal and superconducting state in the limit $R_{N} \gg R_{Q}$ and $C \gg C_{g}$ (for example, see [7]). But to the best of our knowledge, there is no theory to predict $E_{a}$ for $1 \mathrm{D}$ arrays in the limit 


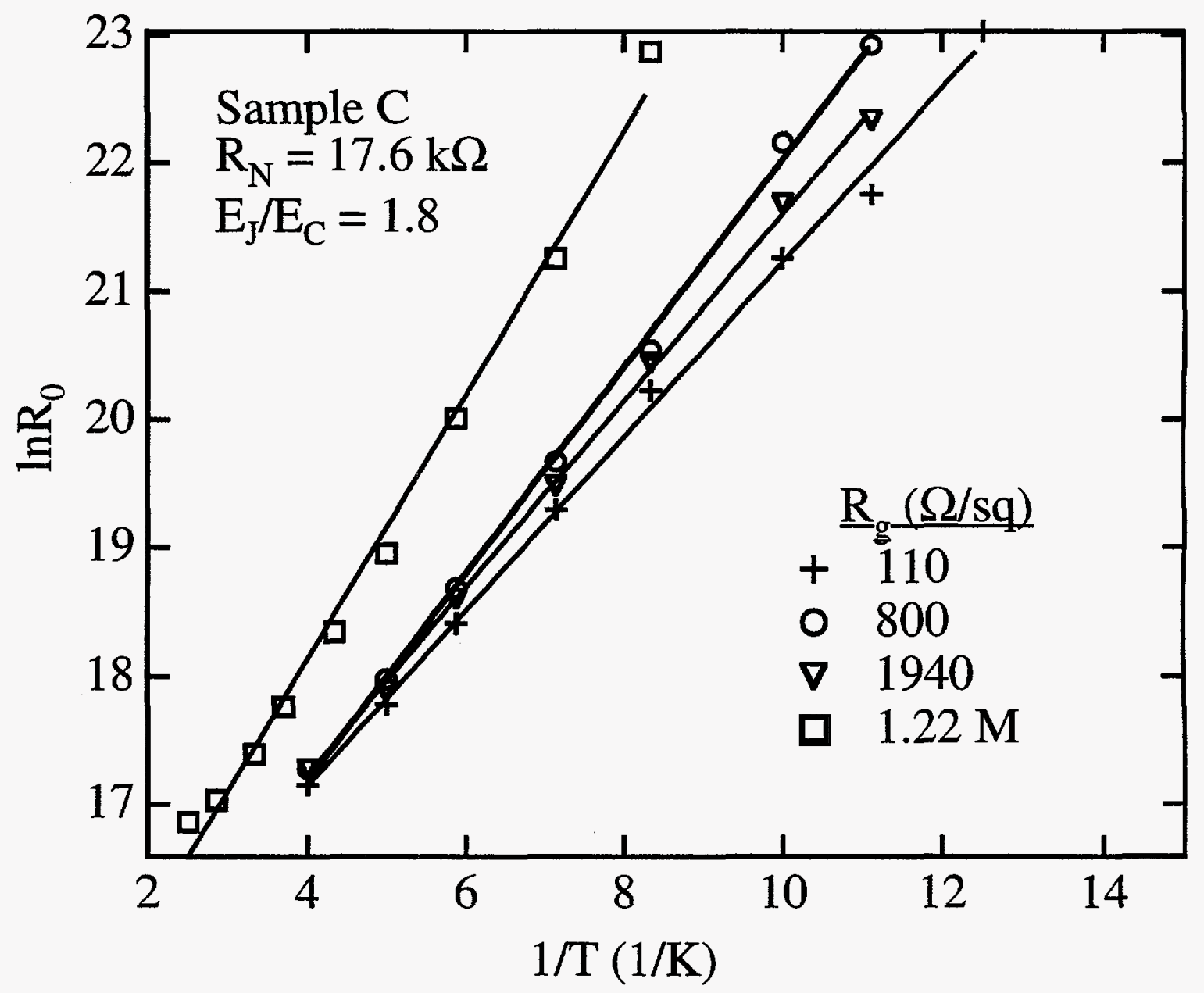

Figure 13.10: Determination of the activation energy $E_{a}$ for sample $\mathrm{C}$ with the islands of the array in the superconducting state (zero applied magnetic field). Shown is $\ln R_{0}$ versus inverse temperature for four ground plane resistances $R_{g}=110,800,1940$, and 1.22 $\mathrm{M} \Omega / \mathrm{sq}$. The symbols are the data points, and the lines are linear fits to the data, giving $E_{a}$ for the expression $R_{0}=R_{1} \exp \left(E_{a} / k_{B} T\right)$. The fitted activation energies for the four different ground plane resistances are $E_{a}=0.68,0.73,0.81$, and 1.0 , respectively. 
$C_{g}>C$. But it is interesting to compare our results to the theoretical predictions that do exist.

For $2 \mathrm{D}$ arrays in the limit $R_{N} \gg R_{Q}$ and $C \gg C_{g}$ with the islands in the normal state, conduction is caused by thermal activation of single electron solitons. $E_{a}$ is then given by $1 / 2$ the energy used to create a soliton-antisoliton pair with a separation of one unit cell, $E_{C} / 4$, where the charging energy $E_{C}$ is given in this limit as $e^{2} / 2 C$. In the superconducting state, there can be two transport mechanisms, splitting of Cooper pairs into single electron soliton pairs and the formation of Cooper pair solitons. The former requires an activation energy $E_{a}=E_{C} / 4+\Delta$, where $\Delta$ is the superconducting energy gap. The latter requires an energy $E_{a}=E_{C}[19]$.

As seen in Table 13.2, the activation energy with the islands in the normal state for all three $1 \mathrm{D}$ arrays is smaller than $E_{C}$. For the insulating samples $\mathrm{B}$ and $\mathrm{C}$, at the smallest $R_{g}, E_{a N} \approx E_{C} / 2,3 E_{C} / 4$, respectively. For the superconducting sample $\mathrm{A}, E_{a N} \approx E_{C} / 13$. The activation energy with the islands in the superconducting state for samples $B$ and $C$ (sample A did not show thermally activated behavior with the islands in the superconducting state) at the smallest $R_{g}$ are $E_{a S} \approx 2.4 E_{C}$ and $3 E_{C}$, respectively.

\subsection{Conclusion}

In conclusion, we have presented measurements of three $1 \mathrm{D}$ arrays of submicron Josephson junctions capacitively coupled to a 2DEG. We use the 2DEG as a source of dissipation for the array by varying its resistance per square $R_{g}$ via a back gate voltage $V_{B G}$. Measurements of the capacitance to ground $C_{g}$ of the islands indicate it is independent of $V_{B G}$, so that changing $V_{B G}$ changes only the dissipation and no other relevant physical parameters. At the smallest value of ground plane resistance $R_{g}$ (largest dissipation), the array with ratio of Josephson energy to charging energy $E_{J} / E_{C} \approx 6$ displays superconductorlike behavior, and the arrays with $E_{J} / E_{C} \approx 2$ display insulator-like behavior. Therefore, a superconductor-like to insulator-like transition as a function of $E_{J} / E_{C}$ occurs in the range $2<E_{J} / E_{C}<6$. As the dissipation is decreased by increasing $R_{g}$, the zero-bias resistance $R_{0}$ increases in all three arrays with the islands in both the superconducting and normal states. For the superconducting array, the size of the supercurrent increases, reaches a maximum, and then decreases, as $R_{g}$ increases. For the insulating arrays, the size of the charging gap increases with increasing $R_{g}$. We should be able to tune an array 
with a ratio $E_{J} / E_{C} \approx 4$ through a superconductor-like to insulator-like transition by increasing the ground plane resistance $R_{g}$ and thereby decreasing the dissipation in the local electrodynamic environment. 


\section{Chapter 14}

\section{Conclusions and Future Directions}

\subsection{Conclusions}

In this dissertation we presented the results of low temperature measurements of arrays of $\mathrm{Al}$ islands linked by submicron $\mathrm{Al} / \mathrm{Al}_{\mathrm{x}} \mathrm{O}_{\mathrm{y}} / \mathrm{Al}$ tunnel junctions over a ground plane. One-dimensional (1D) and two-dimensional (2D) arrays were fabricated on substrates with a conducting region, a ground plane, separated from the array by an insulating layer.

We studied a $1 \mathrm{D}$ and $2 \mathrm{D}$ array of metallic islands linked by tunnel junctions with normal state tunneling resistance much larger than the resistance quantum for single electrons, $R_{N} \gg R_{Q e}$. The arrays were fabricated over a ground plane, a degenerately-doped silicon substrate topped with approximately $100 \mathrm{~nm}$ of oxide, such that the capacitance to ground of the islands was much larger than the junction capacitance, $C_{g} \gg C$. The current-voltage $(I-V)$ characteristics of the arrays exhibit a threshold voltage $V_{T}$ below which there is almost no conduction and above which the current through the array scales as the reduced voltage $v=V / V_{T}-1\left(I \sim v^{\zeta}\right)$. The values of the threshold voltages $V_{T}$ and scaling exponent $\zeta$ in the $1 \mathrm{D}$ and $2 \mathrm{D}$ array are in qualitative agreement with the simulations of Middleton and Wingreen [63] who interpreted the threshold behavior as a dynamic critical phenomenon. A 2D array with intentionally-added island area disorder was also measured. The $I-V$ characteristics of the array with added area disorder are different from those for the array with only intrinsic disorder. The sizes of the Coulomb blockade regions of the two arrays are approximately the same, but the threshold voltage for the array with area disorder is less well-defined. The scaling law is obeyed over a narrower range for the array with area disorder, and the scaling exponent for the array with area 
disorder is nearly $50 \%$ larger than for the array with intrinsic disorder.

We studied a $2 \mathrm{D}$ array of superconducting islands linked by Josephson junctions with normal state tunneling resistance close to the resistance quantum for Cooper pairs, $R_{N} \approx R_{Q}$, and $C_{g} \gg C$ such that the Josephson and charging energies of the array were approximately equal, $E_{J} / E_{C} \approx 1$. The array was fabricated over a ground plane with variable dissipation, a $\mathrm{GaAs} / \mathrm{Al}_{0.3} \mathrm{Ga}_{0.7} \mathrm{As}$ heterostructure with a two-dimensional electron gas (2DEG) located approximately $100 \mathrm{~nm}$ from the surface. By applying a large voltage between the 2DEG and a metallic back gate, the sheet density, and thus the resistance per square $R_{g}$, of the 2DEG could be changed in situ. By changing $R_{g}$, we change the dissipation associated with the local electrodynamic environment of the array without changing any other relevant parameters of the system. The $I-V$ characteristics of the array change from superconductor-like to insulator-like as $R_{g}$ is increased. The zero-bias resistance $R_{0}$ of the array versus temperature curves change from superconductor-like with quasi-reentrant behavior to insulator as $R_{g}$ is increased, with $R_{0}$ increasing exponentially with $R_{g}$. Our results agree reasonably well with the theoretical predictions of Wagenblast et al. [93] describing a transition from capacitive to dissipative dynamics of the superconducting phases of the array as $R_{g}$ is increased. We also investigate the effects of a small magnetic field applied perpendicularly to the array. As the frustration $f$ (number of flux quanta per unit cell of the array) is increased, the $I-V$ characteristics of the array make a transition from superconductor-like to insulator-like. The $R_{0}$ versus temperature curves make a transition from superconductor-like with quasi-reentrant behavior to insulator as $f$ is increased. Following the work of Fisher [33], we analyze the scaling behavior of the magnetic field-driven superconductor-to-insulator transition and do not find good agreement with the predicted behavior. We also investigate the effects of varying both $f$ and $R_{g}$ simultaneously. We find that increasing $R_{g}$ drives the magnetic field-driven S-I transition to lower values of $f$, and increasing $f$ drives the dissipation-driven transition to lower values of $R_{g}$.

Three 1D arrays of superconducting islands linked by Josephson junctions with $R_{N} \approx R_{Q}$ and $C_{g} \gg C\left(E_{J} / E_{C} \approx 1\right)$ over a ground plane with variable dissipation were studied. One array displays superconducting behavior at low temperature. As $R_{g}$ is increased from its smallest value, the supercurrent feature becomes larger in size; at $R_{g} \approx 12 \mathrm{k} \Omega / \mathrm{sq}$ the size of the supercurrent reaches a maximum, and for $R_{g} \geq 12 \mathrm{k} \Omega / \mathrm{sq}$ the supercurrent size decreases and the hysteresis in the $I-V$ characteristic disappears. Two of the arrays display insulating behavior at low temperature, with a Coulomb gap 
feature. As the resistance of the ground plane $R_{g}$ is increased, the size of the Coulomb gap increases. In general, the zero-bias resistance $R_{0}$ of the arrays increases with increasing $R_{g}$. In contrast to the $2 \mathrm{D}$ array, the increase of $R_{0}$ with $R_{g}$ is stronger than exponential.

\subsection{Future Directions}

Many experiments remain to be performed on arrays of tunnel junctions over a ground plane in the limit $C_{g} \gg C$. We would like to continue our studies of both the arrays of normal metal islands linked by tunnel junctions in the limit $R_{N} \gg R_{Q e}$ and the arrays of superconducting islands linked by Josephson junctions with $R_{N} \approx R_{Q}$ over a ground plane with variable dissipation.

With the arrays of normal metal islands in the limit $R_{N} \gg R_{Q e}$, we would like to measure both $1 \mathrm{D}$ and 2D arrays of different sizes (the theory of Middleton and Wingreen predict that the threshold voltage of conduction should scale with array size). We would like to investigate more carefully the effects of temperature on the $I-V$ characteristics and zero-bias resistance of the arrays. We would also like to continue our study of $2 \mathrm{D}$ arrays with intentionally-added island area disorder, by measuring more arrays with different disorder realizations and arrays of different sizes.

With the arrays of superconducting islands linked by Josephson junctions with $R_{N} \approx R_{Q}$ over a ground plane with variable dissipation, we would like to measure both $1 \mathrm{D}$ and $2 \mathrm{D}$ arrays of different sizes. For the $1 \mathrm{D}$ arrays, we would like to measure a sample that can be driven through a dissipation-driven superconductor-like to insulator-like transition, as was observed in the $2 \mathrm{D}$ array. We would also like to investigate the effects of varying the dissipation due to a ground plane in a zero-dimensional system, that is, a single electron transistor (one superconducting island with two Josephson junctions). Work on both the dissipation-driven transition in a 1D array and the single electron transistor is in progress as of the writing of this dissertation. 


\section{Appendix A}

\section{Preparation of}

\section{Degenerately-Doped Silicon \\ Substrates}

1. Strip original oxide ${ }^{1}\left(\mathrm{SiO}_{2}\right)$ from wafer and grow $80 \mathrm{~nm}$ thermal oxide.

(a) Strip original oxide in a buffered HF (BHF) $\left(6: 1 \mathrm{NH}_{4}: \mathrm{HF}\right)$ etch (5:1 or 10:1 $\mathrm{H}_{2} 0:$ BHF, $\sim 1$ min. 45 sec.).

(b) Clean in pirahna etch $\left(1: 5 \mathrm{H}_{2} \mathrm{O}_{2}: \mathrm{H}_{2} \mathrm{SO}_{4}, 10 \mathrm{~min}\right.$.).

(c) Strip native oxide in $\mathrm{HF}\left(25: 1 \mathrm{H}_{2} \mathrm{O}: \mathrm{HF}, \sim 45 \mathrm{sec}\right.$.).

(d) Grow $80 \mathrm{~nm}$ thermal oxide in a Tylan Atmospheric Furnace.

2. Etch holes for contact pads in oxide.

(a) Coat wafer with KTI (Kodak) Positive 820 photoresist and bake using the Eaton LSI 45/60 Wafer Processing Station.

(b) Expose photoresist with contact pads pattern (using a custom-designed mask made at the Microfabrication Laboratory) using the GCA 6200 Wafer Stepper.

(c) Develop photoresist with KTI Positive Developer using the MTI Omnichuck Development Station.

\footnotetext{
${ }^{1}$ We removed the original $100 \mathrm{~nm}$ of $\mathrm{SiO}_{2}$ because we were unsure of its quality and uniformity.
} 
(d) Remove photoresist residue ("descum") using the Technics C Plasma Etching System.

(e) Bake in $120^{\circ} \mathrm{C}$ oven $1 \mathrm{hr}$.

(f) Etch contact pad pattern in oxide in BHF (10:1 $\mathrm{H}_{2} \mathrm{O}: \mathrm{BHF}, \sim 1 \mathrm{~min} .30 \mathrm{sec}$.).

(g) Strip off resist with acetone using the MTI.

3. Grow $55 \mathrm{~nm}$ oxide in contact pad region and increase non-pad oxide region to 100 nm.

(a) Clean in pirahna.

(b) Etch pad oxide in HF (25:1 $\mathrm{H}_{2} 0: \mathrm{HF}, \sim 45 \mathrm{sec}$.).

(c) Grow $55 \mathrm{~nm}$ oxide in pad area (while increasing non-pad oxide to $100 \mathrm{~nm}$ ) using a Tylan Furnace.

4. Implant and activate arsenic in contact pad region.

(a) Coat wafer with KTI (Kodak) Positive 820 photoresist and bake using the Eaton LSI 45/60 Wafer Processing Station.

(b) Expose photoresist with contact pads pattern using the GCA 6200 Wafer Stepper.

(c) Develop photoresist with KTI Positive Developer using the MTI.

(d) Descum using the Technics C.

(e) Bake in $120^{\circ} \mathrm{C}$ oven $1 \mathrm{hr}$.

(f) Send to Ion Implant Services for As ion implantation $\left(160 \mathrm{keV}, 5 \times 10^{15} \mathrm{~cm}^{-2}, 7^{\circ}\right.$ incidence angle).

(g) Remove photoresist using oxygen plasma ("plasma ash resist") using the Technics C.

(h) Clean in pirahna.

(i) Anneal ion implants in a Tylan Furnace.

(j) Coat wafer with KTI (Kodak) Positive 820 photoresist and bake using the Eaton LSI 45/60 Wafer Processing Station.

(k) Expose photoresist with contact pads pattern using the GCA 6200 Wafer Stepper.

(l) Develop photoresist with KTI Positive Developer using the MTI. 
(m) Descum using the Technics C.

(n) Bake in $120^{\circ} \mathrm{C}$ oven $1 \mathrm{hr}$.

(o) Etch oxide in pad areas in BHF (10:1 $\mathrm{H}_{2} \mathrm{O}: \mathrm{BHF}, \sim 1 \mathrm{~min} .30 \mathrm{sec}$.).

(p) Strip photoresist with acetone using the MTI.

(q) Clean in pirahna.

5. Sputter and sinter $100 \mathrm{~nm}$ aluminum in contact pad areas.

(a) Coat wafer with Shipley 1400-31 photoresist and bake using the Eaton.

(b) Soak in chlorobenzene (10 min.).

(c) Expose photoresist with contact pads pattern using the GCA 6200 Wafer Stepper.

(d) Develop photoresist with Shipley Microposit Developer using the MTI.

(e) Descum using the Technics C.

(f) Etch oxide in pad areas in BHF (25:1 $\mathrm{H}_{2} \mathrm{O}: \mathrm{BHF}, \sim 45 \mathrm{sec}$.).

(g) Immediately sputter $100 \mathrm{~nm} \mathrm{Al} /(2 \% \mathrm{Si})$ using the CPA 9900 Sputtering System.

(h) Remove photoresist and $\mathrm{Al} /(2 \% \mathrm{Si})$ on photoresist by soaking in acetone overnight ("liftoff").

(i) Clean with oxygen plasma using Technics C.

(j) Sinter contacts in a Tylan Furnace.

6. Cut and clean substrates.

(a) Coat wafer with KTI photoresist and bake using the Eaton.

(b) Apply dicing tape to back side of the wafer.

(c) Dice wafer into $1 \mathrm{~cm}^{2}$ pieces using the Disco Automatic Dicing Saw.

(d) Remove substrates from dicing tape.

(e) Remove photoresist by soaking in acetone $(10 \mathrm{~min}$.).

(f) Ultrasound in RBS soap (5:1 $\mathrm{H}_{2} \mathrm{O}: \mathrm{RBS}, 10 \mathrm{~min}$.).

(g) Ultrasound in acetone (10 min.).

(h) Ultrasound in methanol (10 min.). 


\section{Appendix B}

\section{Electron-Beam Lithography Exposure Procedure}

1. Place a small amount of silver powder suspended in methanol on two opposite sides of the resist-coated substrate (near the edges). Blow dry with nitrogen gas.

2. Clip substrate to scanning electron microscope (JEOL 6400 SEM) sample mount and load sample mount into SEM. Set working distance $(8 \mathrm{~mm})$.

3. Turn on accelerating voltage $(20 \mathrm{kV})$ and filament current $\left(\mathrm{LaB}_{6}\right.$ cathode, $\left.\sim 1.9 \mathrm{~A}\right)$.

4. Adjust gun tilt and shift to maximize beam current.

5. Adjust beam current to approximately the value to be used in the first exposure (10 $\mathrm{pA}$ ).

6. Let filament warm up $1 \mathrm{hr}$.

7. Adjust gun tilt and shift to maximize beam current.

8. Adjust focus and astigmatism on silver powder pieces on one side of substrate.

9. Adjust focus on silver powder on opposite side of substrate.

10. Find center of substrate (exposure location) by determining coordinates of opposite corners and interpolating. 
11. Determine focus to be used at center of substrate by interpolating focus values determined at opposite sides of substrate.

12. Adjust beam current to value to be used in first exposure (10 pA, aperture 4). Move to center and set focus and magnification $(300 \times)$ for first exposure.

13. Put in Probe Current Detector (PCD). Move to center of substrate.

14. Take PCD out. Expose resist/substrate with first pattern (array pattern). Put PCD in.

15. Change beam current (200 pA, aperture 3 ) and magnification $(100 \times)$ for second exposure.

16. Take PCD out. Expose resist/substrate with second pattern (intermediate leads pattern). Put PCD in.

17. Change beam current ( $6 \mathrm{nA}$, aperture 2$)$ and magnification $(25 \times)$ for third exposure.

18. Take PCD out. Expose resist/substrate with third pattern (contact pads pattern). Put PCD in.

19. Turn down filament current and accelerating voltage. Remove sample mount from SEM, and remove substrate from sample mount. 


\section{Appendix C}

\section{Two-Dimensional Josephson Junction Arrays and the XY Model}

The part of the Hamiltonian of a 2D Josephson junction array due to the Josephson energy:

$$
E_{J} \sum_{i, j} \cos \left(\phi_{i}-\phi_{j}\right)
$$

resembles the Hamiltonian for the $2 \mathrm{D} X Y$ model [64]. The XY model consists of spins constrained to rotate in the plane of a $2 \mathrm{D}$ lattice. Only the interaction of nearest neighbors is considered. The interaction energy between spin $i$ and $j$ is given by:

$$
U_{i j}=-J \cos \left(\phi_{i}-\phi_{j}\right)
$$

where $\mathrm{J}$ is a temperature-independent positive constant and $\phi_{i}$ is the angle that spin $i$ makes with a reference direction.

At $T=0$, the spins are all parallel. At finite temperatures, "spin waves", large length variations in $\phi$, occur. At higher $T$, topological excitations called vortices start to occur. Going around one vortex (anti-vortex), the phase changes by $2 \pi(-2 \pi)$. In a $2 \mathrm{D}$ Josephson junction array, a vortex (anti-vortex) is a supercurrent moving in a counterclockwise (clockwise) direction [19] which gives rise to a quantized magnetic flux:

$$
\Phi= \pm \Phi_{0} \equiv \pm \frac{h}{2 e}
$$




\section{Bibliography}

[1] B. Abeles, Phys. Rev. B 15, 2828 (1977).

[2] V. Ambegaokar, U. Eckern, and G. Schön, Phys. Rev. Lett. 48, 1745 (1982).

[3] V. Ambegaokar and A. Baratoff, Phys. Rev. Lett. 10, 486 (1963).

[4] D. V. Averin and K. K. Likharev, Mesoscopic Phenomena in Solids, edited by B. L. Altshuler, P. A. Lee, and R. A. Webb, Elsevier Science Publishers, Amsterdam, 1991.

[5] D. V. Averin and Y. V. Nazarov, Single Charge Tunneling: Coulomb Blockade Phenomena in Nanostructures, edited by H. Grabert and M. H. Devoret, Plenum Press, New York, 1992.

[6] N. S. Bakhvalov, G. S. Kazacha, K. K. Likharev, and S. I. Serdyukova, Sov. Phys. JETP 68, 581 (1989).

[7] N. S. Bakhvalov, G. S. Kazacha, K. K. Likharev, and S. I. Serdyukova, Physica B 173, 319 (1991).

[8] S. Bhattacharya and M. J. Higgins, Phys Rev. Lett. 70, 2617 (1993).

[9] P. A. Bobbert, R. Fazio, G. Schön, and G. T. Zimanyi, Phys. Rev. B 41, 4009 (1990).

[10] R. M. Bradley and S. Doniach, Phys. Rev. B 30, 1138 (1984).

[11] C. Bruder, R. Fazio, A. Kampf, A. van Otterlo, and G. Schön, Physica Scripta T42, $182(1992)$.

[12] A. O. Caldeira and A. J. Leggett, Ann. Phys. (N.Y.) 149, 374 (1983).

[13] A. O. Caldeira and A. J. Leggett, Ann. Phys. (N.Y.) 445, 445 (E) (1984). 
[14] A. O. Calderia and A. J. Leggett, Phys. Rev. Lett. 46, 211 (1981).

[15] S. Chakravarty, G.-L. Ingold, S. Kivelson, and A. Luther, Phys. Rev. Lett. 56, 2303 (1986).

[16] S. Chakravarty, S. Kivelson, G. T. Zimanyi, and B. I. Halperin, Phys. Rev. B 35, 7256 (1987).

[17] C. D. Chen, P. Delsing, D. B. Haviland, and T. Claeson, Physica Scripta T42, 182 (1992).

[18] C. D. Chen, P. Delsing, D. B. Haviland, Y. Harada, and T. Claeson, Phys. Rev. B 51, 15645 (1995).

[19] C. Chen, Dynamics of Vortices and Charges in Two Dimensional Arrays of Small Josephson Junctions, PhD thesis, Chalmers University of Technology, 1994.

[20] P. Delsing, Single Charge Tunneling: Coulomb Blockade Phenomena in Nanostructures, edited by H. Grabert and M. H. Devoret, Plenum Press, New York, 1992.

[21] P. Delsing, C. D. Chen, D. B. Haviland, Y. Harada, and T. Claeson, Phys. Rev. B 50, 3959 (1994).

[22] P. Delsing, K. K. Likharev, L. S. Kuzmin, and T. Claeson, Phys. Rev. Lett. 63, 1861 (1989).

[23] M. H. Devoret and H. Grabert, Single Charge Tunneling: Coulomb Blockade Phenomena in Nanostructures, edited by H. Grabert and M. H. Devoret, Plenum Press, New York, 1992.

[24] G. J. Dolan, Appl. Phys. Lett. 31, 337 (1977).

[25] S. Doniach, Phys. Rev. B 24, 5063 (1981).

[26] V. J. Emery and S. A. Kivelson, Phys. Rev. Lett. 74, 3253 (1995).

[27] R. Fazio, A. van Otterlo, and G. Schön, Europhys. Lett. 25, 453 (1994).

[28] R. Fazio and G. Schön, Phys Rev. B 43, 5307 (1991).

[29] D. S. Fisher, Phys. Rev. B 31, 1396 (1985). 
[30] D.S. Fisher, Nonlinearity in Condensed Matter, edited by A. R. Bishop et al., SpringerVerlag, New York, 1987.

[31] M. P. A. Fisher, G. Grinstein, and S. M. Girvin, Phys. Rev. Lett. 64, 587 (1990).

[32] M. P. A. Fisher, Phys. Rev. B 36, 1917 (1987).

[33] M. P. A. Fisher, Phys. Rev. Lett. 65, 923 (1990).

[34] M. G. Forrester, H. J. Lee, M. Tinkham, and C. J. Lobb, Phys. Rev. B 37, 5966 (1988).

[35] B. Geerligs, Classical and Quantum Charge Dynamics in Small Tunnel Junctions, $\mathrm{PhD}$ thesis, Delft University of Technology, 1990.

[36] L. J. Geerligs et al., Phys. Rev. Lett. 63, 326 (1989).

[37] U. Geigenmüller and G. Schön, Europhys. Lett. 10, 765 (1989).

[38] E. Granato and J. M. Kosterlitz, Phys. Rev. B 33, 6533 (1986).

[39] G. Grüner, Rev. Mod. Phys. 60, $1129^{\prime}(1988)$.

[40] D. B. Haviland, Y. Liu, and A. M. Goldman, Phys. Rev. Lett. 62, 2180 (1989).

[41] A. F. Hebard and M. A. Paalanen, Phys. Rev. Lett. 65, 927 (1990).

[42] R. E. Howard, E. L. Hu, L. D. Jackal, L. A. Fetter, and R. H. Bosworth, Appl. Phys. Lett. 35, 879 (1979).

[43] M. Iansiti, A. T. Johnson, C. J. Lobb, and M. Tinkham, Phys. Rev. B 40, 11370 (1988).

[44] M. Iansiti, M. Tinkham, A. T. Johnson, W. F. Smith, and C. J. Lobb, Phys. Rev. B 39, 6465 (1989).

[45] L. Jacobs, J. V. José, and M. A. Novotny, Phys. Rev. Lett. 53, 2177 (1984).

[46] J. M. Jaeger, D. B. Haviland, B. G. Orr, and A. M. Goldman, Phys. Rev. B 40, 182 (1989).

[47] J. V. José and C. Rojas, Physica B 203, 481 (1994). 
[48] B. D. Josephson, Phys. Lett. 1, 251 (1962).

[49] A. Kampf and G. Schön, Phys. Rev. B 36, 3651 (1987).

[50] M. Kardar, G. Parisi, and Y.-C. Zhang, Phys. Rev. Lett. 56, 889 (1986).

[51] B. J. Kim and M. Y. Choi, Phys. Rev. B 52, 3624 (1995).

[52] J. G. Kissner and U. Eckern, Z. Phys. B 91, 155 (1993).

[53] S. E. Korshunov and T. Nattermann, Physica B 222, 280 (1996).

[54] L. D. Landau and E. M. Lifshitz, Electrodynamics of Continuous Media, Pergamon Press, Oxford, 1984.

[55] K. K. Likharev, Dynamics of Josephson Junctions and Circuits, Gordon and Breach Science Publishers, Philadelphia, 1986.

[56] Y. Liu, D. B. Haviland, B. Nease, and A. M. Goldman, Phys. Rev. B 47, 5931 (1993).

[57] C. J. Lobb, D. W. Abraham, and M. Tinkham, Phys. Rev. B 27, 150 (1983).

[58] O. V. Lounasmaa, Experimental Principles and Methods Below 1K, Academic Press, London, 1974.

[59] G. Luciano, U. Eckern, and J. G. Kissner, Europhys. Lett. 32, 669 (1995).

[60] J. M. Martinis, M. H. Devoret, and J. Clarke, Phys. Rev. B 35, 4682 (1987).

[61] N. Martys, M. Cieplak, and M. O. Robbins, Phys. Rev. Lett. 66, 1058 (1991).

[62] D. E. McCumber, J. Appl. Phys. 39, 3113 (1968).

[63] A. A. Middleton and N. S. Wingreen, Phys. Rev. Lett. 71, 3198 (1993).

[64] J. E. Mooij, Percolation, Localization, and Superconductivity, edited by A. M. Goldman and S. A. Wolf, Plenum Press, New York, 1983.

[65] J. E. Mooij et al., Phys. Rev. Lett. 65, 645 (1990).

[66] B. G. Orr, H. M. Jaeger, and A. M. Goldman, Phys. Rev. B 32, 7586 (1985). 
[67] B. G. Orr, H. M. Jaeger, A. M. Goldman, and C. G. Kuper, Phys. Rev. Lett. 56, 378 (1986).

[68] M. A. Paalanen, A. F. Hebard, and R. R. Ruel, Phys. Rev. Lett. 69, 1604 (1992).

[69] W. H. Press, B. P. Flannery, S. A. Teukolsky, and W. T. Vetterling, Numerical Recipes in C, Cambridge University Press, Cambridge, 1990.

[70] R. C. Richardson and E. N. Smith, Experimental Techniques in Condensed Matter Physics at Low Temperatures, Addison Wesley, Redwood City, CA, 1988.

[71] A. J. Rimberg, T. R. Ho, and J. Clarke, Phys. Rev. Lett. 74, 4714 (1995).

[72] A. J. Rimberg et al., Phys. Rev. Lett. 78, 2632 (1997).

[73] A. J. Rimberg, Magnetotransport in Uniform and Modulated Electron Wells in Wide Parabolic Quantum Wells, PhD thesis, Harvard University, 1992.

[74] G. I. Rochlin, Rev. Sci. Inst. 41, 73 (1970).

[75] M. S. Rzchowski, S. P. Benz, M. Tinkham, and C. J. Lobb, Phys. Rev. B 42, 2041 (1990).

[76] J. M. Schmidt, Resonant Tunneling in Small Current-Biased Josephson Junctions, PhD thesis, University of California at Berkeley, 1994.

[77] G. T. Seidler, T. F. Rosenbaum, and B. W. Veal, Phys. Rev. B 45, 10162 (1992).

[78] S. L. Sondhi, S. M. Girvin, J. P. Carini, and D. Shahar, Rev. Mod. Phys. 69, 315 (1997).

[79] W. C. Stewart, Appl. Phys. Lett. 12, 277 (1968).

[80] A. G. Sun et al., Phys. Rev. B 50, 3266 (1994).

[81] S. Tanda, S. Ohzeki, and T. Nakayama, Phys. Rev. Lett. 69, 530 (1992).

[82] T. S. Tighe, M. T. Tuominen, J. M. Hergenrother, and M. Tinkham, Phys. Rev. B 47, 1145 (1993).

[83] T. S. Tighe, Meaurements on Two-Dimensional Arrays of Mesoscopic Josephson Junctions, PhD thesis, Harvard University, 1993. 
[84] M. T. Tuominen, J. M. Hergenrother, T. S. Tighe, and M. Tinkham, Phys. Rev. Lett. 69, 1997 (1992).

[85] L. J. van der Pauw, Philips Techn. Rdsch. 20, 230 (1958/9).

[86] H. S. J. van der Zant, W. J. Elion, L. J. Geerligs, and J. E. Mooij, Phys. Rev. B 54, $10081(1996)$.

[87] H. S. J. van der Zant, F. C. Fritschy, W. J. Elion, L. J. Geerligs, and J. E. Mooij, Phys. Rev. Lett. 69, 2971 (1992).

[88] H. S. J. van der Zant, H. A. Rijken, and J. E. Mooij, J. Low Temp. Phys. 79, 289 (1990).

[89] H. van der Zant, Vortex Dynamics in Artificially Fabricated Superconducting Networks, PhD thesis, Delft University of Technology, 1991.

[90] A. van Otterlo, K.-H. Wagenblast, R. Fazio, and G. Schön, Phys. Rev. B 48, 3316 (1993).

[91] A. van Oudenaarden, S. J. K. Várdy, and J. E. Mooij, Phys. Rev. Lett. 77, 4257 (1996).

[92] B. V. van Wees, Phys. Rev. B 44, 2264 (1991).

[93] K.-H. Wagenblast, A. van Otterlo, G. Schön, and G. T. Zimányi, Accepted to Phys. Rev. Lett. (1997).

[94] M. Wallin, E. Sørensen, S. M. Girvin, and A. P. Young, Phys. Rev. B 49, 12115 (1994).

[95] G. K. White, Experimental Techniques in Low-Temperature Physics, Oxford University Press, Oxford, 1987. 